\title{
Sharp asymptotics of the first exit point density
}

\author{
Giacomo Di Gesù ${ }^{* \dagger}$, Tony Lelièvre ${ }^{\dagger}$, Dorian Le Peutrec ${ }^{\ddagger}$ and Boris Nectoux* ${ }^{* \dagger}$
}

January 17, 2019

\begin{abstract}
We consider the exit event from a metastable state for the overdamped Langevin dynamics $d X_{t}=-\nabla f\left(X_{t}\right) d t+\sqrt{h} d B_{t}$. Using tools from semiclassical analysis, we prove that, starting from the quasi stationary distribution within the state, the exit event can be modeled using a jump Markov process parametrized with the Eyring-Kramers formula, in the small temperature regime $h \rightarrow 0$. We provide in particular sharp asymptotic estimates on the exit distribution which demonstrate the importance of the prefactors in the Eyring-Kramers formula. Numerical experiments indicate that the geometric assumptions we need to perform our analysis are likely to be necessary. These results also hold starting from deterministic initial conditions within the well which are sufficiently low in energy. From a modelling viewpoint, this gives a rigorous justification of the transition state theory and the Eyring-Kramers formula, which are used to relate the overdamped Langevin dynamics (a continuous state space Markov dynamics) to kinetic Monte Carlo or Markov state models (discrete state space Markov dynamics). From a theoretical viewpoint, our analysis paves a new route to study the exit event from a metastable state for a stochastic process.
\end{abstract}

\section{Contents}

1 Motivation and presentation of the results 3

1.1 Overdamped Langevin dynamics and metastability . . . . . . . . 4

1.2 From the potential function to a jump Markov process . . . . . . . 4

1.3 Review of the mathematical literature on the Eyring-Kramers formula . 6

1.4 Quasi stationary distribution .................. 8

1.4.1 Definition and a first property of quasi stationary distributions . 8

1.4.2 An eigenvalue problem related to the quasi stationary distribution 9

1.4.3 Back to the jump Markov process . . . . . . . . . . . 10

1.5 Statement of the main result . . . . . . . . . . . . . 12

1.5.1 Agmon distance . . . . . . . . . . . . . . . . 12

1.5.2 Notations and hypotheses . . . . . . . . . . . . 13

1.5.3 Main result . . . . . . . . . . . . . . . . 15

${ }^{*}$ Current affiliation: Institut für Analysis und Scientific Computing, E101-TU Wien, Wiedner Hauptstr. 8, 1040 Wien, Austria. E-mail: \{giacomo.di.gesu,boris.nectoux\}@asc.tuwien.ac.at

${ }^{\dagger}$ CERMICS, École des Ponts, Université Paris-Est, INRIA, 77455 Champs-sur-Marne, France. Email: tony.lelievre@enpc.fr

${ }^{\ddagger}$ Laboratoire de Mathématiques d'Orsay, Univ. Paris-Sud, CNRS, Université Paris-Saclay, 91405 Orsay, France. E-mail: dorian.lepeutrec@math.u-psud.fr 
1.6 Discussion and generalizations . . . . . . . . . . . . . . 17

1.6.1 Extension of the result to other initial conditions . . . . . . 17

1.6.2 On the geometric assumptions $(20), 21)$ and $(26) \ldots \ldots$

1.6.3 Extension of the results to a subset of generalized saddle points and to more general subsets of $\partial \Omega \ldots \ldots \ldots \ldots$

1.7 Strategy for the proof of Theorem 1 and outline of the paper . . . 25

2 General setting and strategy for the proof of Theorem 1

2.1 Witten Laplacians . . . . . . . . . . . . . . . . . . . . . . 26

2.1.1 Notations for Sobolev spaces . . . . . . . . . . . . . 26 26

2.1.2 Witten Laplacians on a manifold with boundary . . . . . . . 27

2.1.3 Small eigenvalues of $\Delta_{f, h}^{D,(0)}(\Omega)$ and $L_{f, h}^{D,(0)}(\Omega) \ldots \ldots \ldots \ldots$

2.2 Statement of the assumptions required for the quasi-modes . . . . . . 31

2.2.1 Assumptions on quasi-modes for $L_{f, h}^{D,(p)}, p \in\{0,1\} \ldots \ldots$. . . 31

2.2.2 Assumptions on quasi-modes for $\Delta_{f, h}^{D,(p)}, p \in\{0,1\} \ldots \ldots$

2.3 Proof of Proposition $25 \ldots \ldots \ldots \ldots$

2.3.1 Gram-Schmidt orthonormalization . . . . . . . . . . 34

2.3.2 Estimates on the interaction terms $\left(\left\langle\nabla u_{h}, \psi_{j}\right\rangle_{L_{w}^{2}}\right)_{j \in\{1, \ldots, n\}} \ldots \ldots 37$

2.3.3 Estimates on the boundary terms $\left(\int_{\Sigma_{k}} \psi_{j} \cdot n e^{-\frac{2}{h} f} d \sigma\right)_{(j, k) \in\{1, \ldots, n\}^{2}} 37$

2.3.4 Estimates on $\left(\int_{\Sigma_{k}}\left(\partial_{n} u_{h}\right) e^{-\frac{2}{h} f} d \sigma\right)_{k \in\{1, \ldots, n\}} \ldots \ldots \ldots \ldots$

3 On the Agmon distance 39

3.1 The set $A(x, y)$ and an equivalent definition of the Agmon distance . . . 40

3.2 First properties of the Agmon distance . . . . . . . . . . . . 45

3.2.1 Upper bounds on $d_{a}$ and topology of $\left(\bar{\Omega}, d_{a}\right) \ldots \ldots \ldots \ldots$

3.2 .2 A lower bound on the Agmon distance . . . . . . . . . 48

3.3 Agmon distance near critical points of $f$ or $\left.f\right|_{\partial \Omega}$ and eikonal equation . $\quad 50$

3.3.1 The Agmon distance near critical points of $f \ldots \ldots . . . .55$

3.3.2 The Agmon distance near critical points of $\left.f\right|_{\partial \Omega} \ldots \ldots \ldots$

3.4 Curves realizing the Agmon distance . . . . . . . . . . . 555

3.4 .1 Preliminary results . . . . . . . . . . . . . . 55

3.4 .2 Proof of Theorem $3 \ldots \ldots \ldots \ldots$

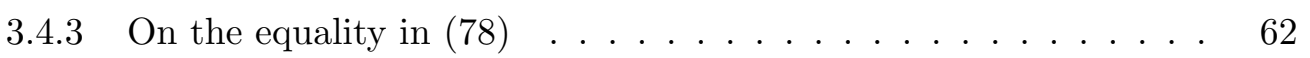

3.5 Agmon distance in a neighborhood of the basin of attraction of a local minimum of $\left.f\right|_{\partial \Omega}$ and eikonal equation . . . . . . . . . . . 66

4 Construction of the quasi-modes and proof of Theorem 1

4.1 Geometric setting and definition of the Witten Laplacians with mixed boundary conditions . . . . . . . . . . . . . . 72

4.1.1 Trace estimates for differential forms and Witten Laplacians on Lipschitz domain with mixed boundary conditions . . . . . . . 72

4.1.2 Construction of the domain $\dot{\Omega}_{i} \ldots \ldots \ldots \ldots$. . . . . . 79

4.1.3 On the spectrum of the Witten Laplacian $\Delta_{f, h}^{M}\left(\dot{\Omega}_{i}\right) \ldots \ldots$. . 87

4.2 Definition of the quasi-modes . . . . . . . . . . . 92

4.2.1 Definition of the quasi-mode $\tilde{u} \ldots \ldots \ldots \ldots$. . . . . . . . . . . . . .

4.2.2 Definition of the quasi-mode $\tilde{\phi}_{i}$ attached to $z_{i} \ldots \ldots \ldots$ 
4.3 Agmon estimates on $u_{h, i}^{(1)} \ldots \ldots \ldots \ldots \ldots \ldots$

4.4 Comparison of the eigenform $u_{h, i}^{(1)}$ and its WKB approximation . . . . 101 4.4.1 Construction of the WKB expansion of $u_{h}^{(1)} \ldots \ldots \ldots$. . . . 102

4.4.2 A first estimate of the accuracy of the WKB approximation . . . 107

4.4.3 A more accurate comparison on the WKB approximation . . . . 110

4.5 Proof of Theorem 1. . . . . . . . . . . . . . . . . 118

5 Consequences and generalizations of Theorem 1

5.1 Proofs of Proposition 12, Proposition 13, Corollary 14 and Corollary 16 125

5.1 .1 Proof of Proposition $12 \ldots \ldots \ldots$. . . . . . . . 125

5.1 .2 Proof of Proposition $13 \ldots \ldots \ldots \ldots$

5.1 .3 Proof of Corollary $14 . \ldots \ldots \ldots \ldots$

5.1 .4 Proof of Corollary $16 \ldots \ldots$. . . . . . . . . . . 128

5.2 Proofs of Theorem 2 and Corollary 17 . . . . . . . . . . . . . 131

5.2.1 Statement of the assumptions required for the quasi-modes . . . 131

5.2 .2 Proof of Proposition $96 \ldots \ldots$. . . . . . . . . . 133

5.2.3 Construction of the quasi-modes which satisfy the estimates of Proposition $96 \ldots \ldots \ldots$

5.2 .4 Proof of Corollary $17 . \ldots \ldots \ldots$

\section{Motivation and presentation of the results}

In materials science, biology and chemistry, atomistic models are now used on a daily basis in order to predict the macroscopic properties of matter from a microscopic description. The basic ingredient is a potential energy function $f: \mathbb{R}^{d} \rightarrow \mathbb{R}$ which associates to a set of coordinates of particles the energy of the system. In practice, $d$ is very large, since the system contains many particles (from tens of thousands to millions).

Using this function $f$, two types of models are built: continuous state space Markov models (stochastic differential equations), such as the Langevin or overdamped Langevin dynamics, and discrete state space Markov models (jump Markov processes). The objective of the analysis presented in this work is to make a rigorous link between these two types of approaches, and in particular to provide a justification of the use of Eyring-Kramers laws to parameterize jump Markov models, by studying the exit event from a metastable state for the overdamped Langevin dynamics.

Jump Markov models are used by practitioners for many reasons. From a modelling viewpoint, new insights can be gained by building such coarse-grained models, that are easier to handle than a large-dimensional stochastic differential equation. From a numerical viewpoint, it is possible to simulate a jump Markov model over timescales which are much larger than the original Langevin dynamics. Moreover, there are many algorithms which use the underlying jump Markov model in order to accelerate the sampling of the original dynamics $76,79,80$.

This section is organized as follows. First, the two models under consideration are introduced, namely the overdamped Langevin dynamics in Section 1.1, and the underlying jump Markov process in Section 1.2. Next, Section 1.3 is devoted to a review of the mathematical literature dealing with metastable processes and the exit event from a metastable state. In Section 1.4, the notion of quasi stationary distribution 
is reviewed. This is a crucial tool in our analysis, in order to connect the overdamped Langevin dynamics to a jump Markov process. Then, in Section 1.5, our main result (Theorem 1) is stated. In Section 1.6, we generalize Theorem 1 in various directions and discuss the geometric assumptions used to state Theorem 1. Finally, in Section 1.7 . we give an outline of the proof of Theorem 1, together with the general organization of the paper.

\subsection{Overdamped Langevin dynamics and metastability}

The continuous state space Markov model we consider in this work is the so-called overdamped Langevin dynamics in $\mathbb{R}^{d}$

$$
d X_{t}=-\nabla f\left(X_{t}\right) d t+\sqrt{h} d B_{t}
$$

driven by the potential function $f: \mathbb{R}^{d} \rightarrow \mathbb{R}$. We assume in the following that $f$ is a $C^{\infty}$ Morse function (all the critical points are non degenerate). The parameter $h=2 k_{B} T>0$ is proportional to the temperature $T$ and $\left(B_{t}\right)_{t \geq 0}$ is a standard $d$ dimensional Brownian motion. One henceforth assumes that

$$
\exists h_{0}, \forall h<h_{0}, \int_{\mathbb{R}^{d}} e^{-\frac{2}{h} f(x)} d x<\infty .
$$

The invariant probability measure of (1) is

$$
\frac{e^{-\frac{2}{h} f(x)} d x}{\int_{\mathbb{R}^{d}} e^{-\frac{2}{h} f(y)} d y}
$$

The basic observation which motivates the use of a jump Markov model to obtain a reduced description of the dynamics (1) is the following. In many practical cases of interest in biology, physics or chemistry, the dynamics (1) is metastable, meaning that the process $\left(X_{t}\right)_{t \geq 0}$ remains trapped for very long times in some regions (called metastable states). If a state is metastable, the way the process leaves this state should not depend too much on the way it enters the state. It is thus tempting to introduce an underlying jump process among these metastable states.

Let us consider a region $\Omega \subset \mathbb{R}^{d}$ and the associated exit event from $\Omega$. More precisely, let us introduce

$$
\tau_{\Omega}=\inf \left\{t \geq 0 \mid X_{t} \notin \Omega\right\}
$$

the first exit time from $\Omega$. The exit event from $\Omega$ is fully characterized by the couple of random variables $\left(\tau_{\Omega}, X_{\tau_{\Omega}}\right)$. The focus of this work is the justification of a jump Markov process to model the exit event from the region $\Omega$, in the small temperature regime $h \rightarrow 0$.

\subsection{From the potential function to a jump Markov process}

The potential function $f$ can also be used to build a jump Markov process to describe the evolution of the system. Jump Markov models are continuous-time Markov processes with values in a discrete state space. In molecular dynamics such processes are known as kinetic Monte Carlo models 81] or Markov state models 7, 72,73. 
Kinetic Monte Carlo models. The basic requirement to build a kinetic Monte Carlo model is a discrete collection of states $D \subset \mathbb{N}$, with associated rates $k_{i, j} \geq 0$ for transitions from state $i$ to state $j$, where $(i, j) \in D \times D$ and $i \neq j$. The neighboring states of state $i$ are those states $j$ such that $k_{i, j}>0$. The dynamics is then given by a jump Markov process $\left(Z_{t}\right)_{t \geq 0}$ with infinitesimal generator $L \in \mathbb{R}^{D \times D}$, where $L_{i, j}=k_{i, j}$ for $i \neq j$.

To be more precise, let us describe how to build the jump process $\left(Z_{t}\right)_{t \geq 0}$ by defining the residence times $\left(T_{n}\right)_{n \geq 0}$ and the subordinated Markov chain $\left(Y_{n}\right)_{n \geq 0}$. Starting at time 0 from a state $Y_{0} \in D$, the model consists in iterating the following two steps over $n \geq 0$ : given $Y_{n}$,

- first sample the residence time $T_{n}$ in $Y_{n}$ as an exponential random variable with parameter $\sum_{j \neq Y_{n}} k_{Y_{n}, j}: \forall i \in D, \forall t>0$,

$$
\mathbb{P}\left(T_{n}>t \mid Y_{n}=i\right)=\exp \left(-\sum_{j \neq i} k_{i, j} t\right),
$$

- and then sample independently from $T_{n}$ the next visited state $Y_{n+1}$ using the following law:

$$
\forall j \neq i, \mathbb{P}\left(Y_{n+1}=j \mid Y_{n}=i\right)=\frac{k_{i, j}}{\sum_{j \neq i} k_{i, j}} .
$$

The continuous time Markov process $\left(Z_{t}\right)_{t \geq 0}$ is then defined as:

$$
\forall n \geq 0, \forall t \in\left[\sum_{m=0}^{n-1} T_{m}, \sum_{m=0}^{n} T_{m}\right), Z_{t}=Y_{n}
$$

with the convention $\sum_{m=0}^{-1} T_{m}=0$.

Transition rates and Eyring Kramers law. Starting from the potential function $f: \mathbb{R}^{d} \rightarrow \mathbb{R}$, one approach to build a kinetic Monte Carlo model is to consider a collection of disjoint subsets $\left(\Omega_{k}\right)_{k \in D}$ which form a partition of the space $\mathbb{R}^{d}$ and to set the transition rates $k_{i, j}$ by considering transitions between these subsets, see for example $9,30,81,82$.

The concept of jump rate between two states is one of the fundamental notions in the modelling of materials. Many papers have been devoted to the rigorous evaluation of jump rates from a full-atom description. The most famous formula is probably the rate derived in the harmonic transition state theory [60,78], which gives an explicit expression for the rate in terms of the underlying potential energy function: this is the Eyring-Kramers formula, that we now introduce.

Let us consider a subset $\Omega$ of $\mathbb{R}^{d}$, which should be thought as one of the subsets $\left(\Omega_{k}\right)_{k \in D}$ introduced above, say the state $k=0$. If $\Omega$ is metastable (in a sense which will be made precise below), it seems sensible to model the exit event from $\Omega$ using a jump Markov model, as introduced in the previous paragraph. As explained above, this requires to define jump rates $\left(k_{0, j}\right)$ from the state 0 to the neighboring states $j$. The aim of this paper is to prove that the exit from $\Omega$ for the dynamics (1) can be approximated using a kinetic Monte-Carlo model with transition rates computed with the Eyring-Kramers formula:

$$
k_{0, j}=A_{0, j} e^{-\frac{2}{h}\left(f\left(z_{j}\right)-f\left(x_{0}\right)\right)}
$$


where $A_{0, j}>0$ is a prefactor, $x_{0}=\arg \min _{x \in \Omega} f(x)$ is the global minimum of $f$ on $\Omega$ which is assumed to be unique and $z_{j}=\arg \min _{z \in \partial \Omega_{j}} f(z)$ where $\partial \Omega_{j}$ denotes the part of the boundary $\partial \Omega$ which connects the region $\Omega$ (numbered 0 ) with the neighboring region numbered $j$ (see Figure 1 for a schematic representation when $\Omega$ has 4 neighboring states).

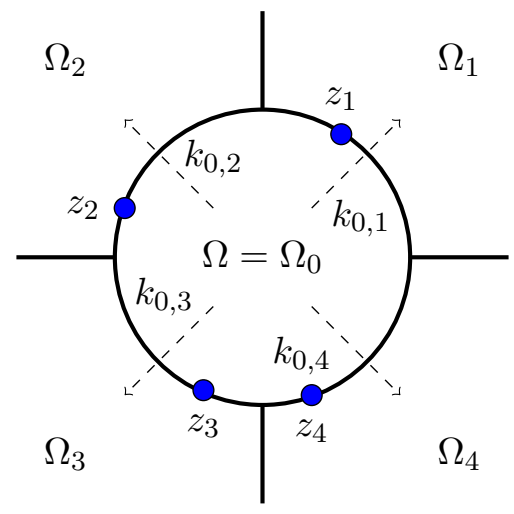

Figure 1: The domain $\Omega=\Omega_{0}$ is associated with state 0 . The neighboring domains $\left(\Omega_{i}\right)_{i=1, \ldots, 4}$ correspond to the four possible next visited states, with associated rates $\left(k_{0, i}\right)_{i=1, \ldots, 4}$. For $i=1, \ldots, 4$, the point $z_{i}$ represent the lowest energy point on $\partial \Omega \cap \partial \Omega_{i}$.

The prefactors $A_{0, j}$ depend on the dynamics under consideration and on the potential function $f$ around $x_{0}$ and $z_{j}$. If $\Omega$ is the basin of attraction of $x_{0}$ for the gradient dynamics $\dot{x}=-\nabla f(x)$ so that the points $z_{j}$ are order one saddle points, the prefactor writes for the overdamped Langevin dynamics (1)

$$
A_{0, j}=\frac{1}{2 \pi}\left|\lambda^{-}\left(z_{j}\right)\right| \sqrt{\frac{\operatorname{det}(\operatorname{Hess} f)\left(x_{0}\right)}{\left|\operatorname{det}(\operatorname{Hess} f)\left(z_{j}\right)\right|}},
$$

where $\lambda^{-}\left(z_{j}\right)$ is the negative eigenvalue of the Hessian of $f$ at $z_{j}$. The formulas (6)-(7) have been obtained by Kramers [49], but also by many authors previously, see the exhaustive review of the literature reported in [34. We also refer to [34] for generalizations to the Langevin dynamics.

\subsection{Review of the mathematical literature on the Eyring-Kramers for- mula}

Let us give the main mathematical approaches to rigorously derive the Eyring-Kramers formula or to study of the exit event from a domain for a stochastic process. See also 2 for a nice review.

Global approaches. Some authors adopt a global approach: they look at the spectrum of the infinitesimal generator of the continuous space dynamics in the small temperature regime $h \rightarrow 0$. It can be shown that there are exactly $m$ small eigenvalues, $m$ being the number of local minima $\left\{x_{1}, \ldots, x_{m}\right\}$ of $f$, and that each of these eigenvalues writes asymptotically when $h \rightarrow 0, A_{i} e^{-\frac{2}{h}\left(f\left(z_{i}\right)-f\left(x_{i}\right)\right)}$, for $i \in\{1, \ldots, m\}$. One thus recovers the form of the Eyring-Kramers formula (6). Here, the saddle point $z_{i}$ attached 
to the local minimum $x_{i}$ is defined by (it is here implicitly assumed that the inf sup value is attained at a single saddle point $z_{i}$ )

$$
f\left(z_{i}\right)=\inf _{\gamma \in \mathcal{P}\left(x_{i}, B_{i}\right)} \sup _{t \in[0,1]} f(\gamma(t))
$$

where $\mathcal{P}\left(x_{i}, B_{i}\right)$ denotes the set of continuous paths from $[0,1]$ to $\mathbb{R}^{d}$ such that $\gamma(0)=x_{i}$ and $\gamma(1) \in B_{i}$ with $B_{i}$ the union of small balls around local minima lower in energy than $x_{i}$. For the dynamics (1), we refer for example to the work [38] based on semiclassical analysis results for Witten Laplacian and the articles [5,6, 26] where a potential theoretic approach is adopted. In the latter results, a connection is made between the small eigenvalues and mean transition times between metastable states. Let us also mention the earlier results $15,16,45,64$. These spectral approaches give the cascade of relevant time scales to reach from a local minimum an other local minimum which is lower in energy. They do not give any information about the typical time scale to go from one local minimum to any other local minimum (say from the global minimum to the second lower minimum for example). These global approaches can be used to build jump Markov models using a Galerkin projection of the infinitesimal generator of $\left(X_{t}\right)_{t \geq 0}$ onto the first $m$ eigenmodes, which gives an excellent approximation of the infinitesimal generator. This has been extensively investigated by Schütte and his collaborators [73], starting with the seminal work 72 .

Local approaches. In this work, we are interested in a local approach, namely the study of the exit event (exit time and exit point) from a fixed given metastable state $\Omega$. The most famous approach to study the exit event in the small temperature limit is the large deviation theory [31]. It relies essentially on the study of slices of the process defined with a suitable sequence of increasing stopping times. In the theory of large deviations, the notion of rate functional is fundamental and gives the cost of deviating from a deterministic trajectory.

In the small temperature regime, large deviation results provide the exponential rates (6), but without the prefactors and without precise error bounds. It can also be proven that the exit time is exponentially distributed in this regime, see [17. For the dynamics (1), a typical result on the exit point distribution is the following (see 31 , Theorem 5.1 in Section 5]): for all $\Omega^{\prime}$ compactly embedded in $\Omega$, for any $\gamma>0$, for any $\delta>0$, there exists $\delta_{0} \in(0, \delta]$ and $h_{0}>0$ such that for all $h<h_{0}$, for all $x \in \Omega^{\prime}$ such that $f(x)<\min _{\partial \Omega} f$, and for all $y \in \partial \Omega$,

$$
\mathrm{e}^{-\frac{2}{h}\left(f(y)-\min _{\partial \Omega} f+\gamma\right)} \leq \mathbb{P}_{x}\left(X_{\tau_{\Omega}} \in \mathcal{V}_{\delta_{0}}(y)\right) \leq \mathrm{e}^{-\frac{2}{h}\left(f(y)-\min _{\partial \Omega} f-\gamma\right)}
$$

where $\mathcal{V}_{\delta_{0}}(y)$ is a $\delta_{0}$-neighborhood of $y$ in $\partial \Omega$. Here and in the following, the subscript $x$ indicates that the stochastic process starts from $x \in \mathbb{R}^{d}: X_{0}=x$.

The strength of large deviation theory is that it is very general: it applies to any dynamics (reversible or non reversible) and in a very general geometric setting, even though it may be difficult in such general cases to make explicit the rate functional, and thus to determine the exit rates. See for example 4,51] for recent contributions to the non reversible case.

Many authors have developed partial differential approach to the same problem. We refer to 20] for a comprehensive review. In particular, formal approaches to study the 
exit time and the exit point distribution have been developed by Matkowsky, Schuss and collaborators in 63, 66, 70, 71] and by Maier and Stein in [58, 59], using formal expansions for singularly perturbed elliptic equations. Some of the results cited above actually consider more general dynamics than (1). Rigorous version of these derivations have been obtained in $21,22,27,45,47,61,62,68$.

Rescaling in time and convergence to a jump process. Finally, some authors prove the convergence to a jump Markov process using a rescaling in time. See for example 48 for a one-dimensional diffusion in a double well, and 32, 62 for a similar problem in larger dimension. In [77, a rescaled in time diffusion process converges to a jump Markov process living on the global minima of the potential $f$, assuming they are separated by saddle points having the same heights. We also refer to the recent review paper [50] for related results.

In this work, we are interested in precise asymptotics of the distribution of $X_{\tau_{\Omega}}$. Our approach is local, justifies the Eyring-Kramers formula (6) with the prefactors and provides sharp error estimates (see (25)). It uses techniques developed in particular in the previous works $38,39,54,56$. Our analysis requires to combine various tools from semiclassical analysis to address new questions: sharp estimates on quasimodes far from the critical points for Witten Laplacians on manifolds with boundary, a precise analysis of the normal derivative on the boundary of the first eigenfunction of Witten Laplacians, and fine properties of the Agmon distance on manifolds with boundary.

Let us finally mention that a summary of the results of this work appeared in [23,55].

\subsection{Quasi stationary distribution}

The quasi stationary distribution is the cornerstone of our analysis. The quasi stationary distribution can be seen as a local equilibrium for a metastable stochastic process when it is trapped in a metastable region. It is actually useful in order to make precise quantitatively what a metastable domain is. In all what follows, we focus on the overdamped Langevin dynamics (1) and a domain $\Omega \subset \mathbb{R}^{d}$. For generalizations to other processes, we refer to $10,11,13$ and in particular to 67 for the spectral analysis of the Langevin dynamics on a bounded domain.

\subsubsection{Definition and a first property of quasi stationary distributions}

Let us first define the quasi stationary distribution.

Definition 1. Let $\Omega \subset \mathbb{R}^{d}$ and consider the dynamics (1). A quasi stationary distribution is a probability measure $\nu_{h}$ supported in $\Omega$ such that

$$
\forall t \geq 0, \nu_{h}(A)=\frac{\int_{\Omega} \mathbb{P}_{x}\left[X_{t} \in A, t<\tau_{\Omega}\right] \nu_{h}(d x)}{\int_{\Omega} \mathbb{P}_{x}\left[t<\tau_{\Omega}\right] \nu_{h}(d x)} .
$$

In words, if $X_{0}$ is distributed according to $\nu_{h}$, then $\forall t>0, X_{t}$ is still distributed according to $\nu_{h}$ conditionally on $X_{s} \in \Omega$ for all $s \in(0, t)$. It is important to notice 
that $\nu_{h}$ is not the invariant measure (2) of the original process restricted to $\Omega$, i.e. $\nu_{h} \neq \mathbf{1}_{\Omega}(x) \frac{e^{-\frac{2}{h} f(x)} d x}{\int_{\mathbb{R}^{d}} e^{-\frac{2}{h} f(y)} d y}$.

In all the following, we will consider that $\Omega$ is a bounded domain in $\mathbb{R}^{d}$. In this context, we have the following results from [53].

Proposition 2. Let $\Omega \subset \mathbb{R}^{d}$ be a bounded domain and consider the dynamics (1). Then, there exists a probability measure $\nu_{h}$ with support in $\Omega$ such that, whatever the law of the initial condition $X_{0}$ with support in $\Omega$,

$$
\lim _{t \rightarrow \infty}\left\|\operatorname{Law}\left(X_{t} \mid t<\tau_{\Omega}\right)-\nu_{h}\right\|_{T V}=0 .
$$

Here and in the following, $\operatorname{Law}\left(X_{t} \mid t<\tau_{\Omega}\right)$ denotes the law of $X_{t}$ conditional to the event $\left\{t<\tau_{\Omega}\right\}$. A corollary of this proposition is that the quasi stationary distribution $\nu_{h}$ exists and is unique.

This proposition also explains why it is relevant to consider the quasi stationary distribution for a metastable domain. The domain $\Omega$ is metastable if the convergence in $(9)$ is much quicker than the exit from $\Omega$. In the following of this paper, we will assume that $\Omega$ is a metastable domain, and we will thus consider the exit event from $\Omega$, assuming that $X_{0}$ is distributed according to the quasi stationary distribution $\nu_{h}$. Let us also mention that we will obtain results starting form deterministic initial conditions $X_{0}=x \in \Omega$ which are sufficiently low in energy (see Section 1.6.1).

\subsubsection{An eigenvalue problem related to the quasi stationary distribution}

In this section, a connection is made between the quasi stationary distribution and an eigenvalue problem for the infinitesimal generator of the dynamics (1)

$$
L_{f, h}^{(0)}=-\nabla f \cdot \nabla+\frac{h}{2} \Delta
$$

with Dirichlet boundary conditions on $\partial \Omega$. In the notation $L_{f, h}^{(0)}$, the superscript (0) indicates that we consider an operator on functions, namely 0 -forms. Here and in the following, we assume that the domain $\Omega$ is a connected open bounded $C^{\infty}$ domain in $\mathbb{R}^{d}$. Let us introduce the weighted $L^{2}$ space

$$
L_{w}^{2}(\Omega)=\left\{u: \Omega \rightarrow \mathbb{R}, \int_{\Omega} u^{2}(x) e^{-\frac{2}{h} f(x)} d x<\infty\right\}
$$

(the weighted Sobolev spaces $H_{w}^{k}(\Omega)$ are defined similarly). The subscript $w$ in the notation $L_{w}^{2}(\Omega)$ and $H_{w}^{k}(\Omega)$ refers to the fact that the weight function $x \in \Omega \mapsto e^{-\frac{2}{h} f(x)}$ appears in the inner product.

The basic observation to define our functional framework is that the operator $L_{f, h}^{(0)}$ is self-adjoint on $L_{w}^{2}(\Omega)$. Indeed, for any smooth test functions $u$ and $v$ with compact supports in $\Omega$, one has

$$
\int_{\Omega}\left(L_{f, h}^{(0)} u\right) v e^{-\frac{2}{h} f}=\int_{\Omega}\left(L_{f, h}^{(0)} v\right) u e^{-\frac{2}{h} f}=-\frac{h}{2} \int_{\Omega} \nabla u \cdot \nabla v e^{-\frac{2}{h} f} .
$$

This gives a proper framework to introduce the Dirichlet realization $L_{f, h}^{D,(0)}(\Omega)$ on $\Omega$ of the operator $L_{f, h}^{(0)}$ : 
Proposition 3. The Friedrichs extension associated with the quadratic form

$$
\phi \in C_{c}^{\infty}(\Omega) \mapsto \frac{h}{2} \int_{\Omega}|\nabla \phi|^{2} e^{-\frac{2}{h} f(x)} d x,
$$

on $L_{w}^{2}(\Omega)$, is denoted $-L_{f, h}^{D,(0)}(\Omega)$. It is a non negative unbounded self adjoint operator on $L_{w}^{2}(\Omega)$ with domain

$$
D\left(L_{f, h}^{D,(0)}(\Omega)\right)=H_{w, 0}^{1}(\Omega) \cap H_{w}^{2}(\Omega)
$$

where $H_{w, 0}^{1}(\Omega)=\left\{u \in H_{w}^{1}(\Omega), u=0\right.$ on $\left.\partial \Omega\right\}$.

The compact injection $H_{w}^{1}(\Omega) \subset L_{w}^{2}(\Omega)$ (which follows from 28 , Theorem 1 in Section 5.7] together with the fact that if a sequence $\left(u_{n}\right)_{n \in \mathbb{N}}$ is bounded in $H_{w}^{1}(\Omega)$ then, $\left(e^{-\frac{1}{h} f} u_{n}\right)_{n \in \mathbb{N}}$ is bounded in $\left.H^{1}(\Omega)\right)$, implies that the operator $L_{f, h}^{D,(0)}(\Omega)$ has compact resolvent. Consequently, its spectrum is purely discrete. Let us introduce $\lambda_{h}>0$ the smallest eigenvalue of $-L_{f, h}^{D,(0)}(\Omega)$. One has the following proposition, which follows from standard results for the first eigenfunction of an elliptic operator, see for example [28, Section 6.3 and Theorem 2 in Section 6.5] and [53, page 128].

Proposition 4. The smallest eigenvalue $\lambda_{h}$ of $-L_{f, h}^{D,(0)}(\Omega)$ is non degenerate and its associated eigenfunction $u_{h}$ has a sign on $\Omega$. Moreover $u_{h} \in C^{\infty}(\bar{\Omega})$.

Without loss of generality, one can assume that:

$$
u_{h}>0 \text { on } \Omega \text { and } \int_{\Omega} u_{h}^{2}(x) e^{-\frac{2}{h} f(x)} d x=1 .
$$

The eigenvalue-eigenfunction couple $\left(\lambda_{h}, u_{h}\right)$ satisfies:

$$
\left\{\begin{aligned}
-L_{f, h}^{(0)} u_{h} & =\lambda_{h} u_{h} \text { on } \Omega, \\
u_{h} & =0 \text { on } \partial \Omega .
\end{aligned}\right.
$$

The link between the quasi stationary distribution $\nu_{h}$ (see Definition 1) and $u_{h}$ is given by the following proposition (see for example [53]):

Proposition 5. The unique quasi stationary distribution $\nu_{h}$ associated with the dynamics (1) and the domain $\Omega$ is given by:

$$
\nu_{h}(d x)=\frac{u_{h}(x) e^{-\frac{2}{h} f(x)}}{\int_{\Omega} u_{h}(y) e^{-\frac{2}{h} f(y)} d y} d x,
$$

where $u_{h}$ is the eigenfunction associated with the smallest eigenvalue of $-L_{f, h}^{D,(0)}(\Omega)$ (see Proposition 4).

\subsubsection{Back to the jump Markov process}

As explained in Section 1.4.1, if the process remains for a sufficiently long time in the domain $\Omega$, it is natural to consider the exit event starting from the quasi stationary distribution attached to $\Omega$. The next proposition characterizes the law of this exit event (see for example [53]). 
Proposition 6. Let us consider the dynamics (1) and the quasi stationary distribution $\nu_{h}$ associated with the domain $\Omega$. If $X_{0}$ is distributed according to $\nu_{h}$, the random variables $\tau_{\Omega}$ and $X_{\tau_{\Omega}}$ are independent. Furthermore, $\tau_{\Omega}$ is exponentially distributed with parameter $\lambda_{h}$ and the law of $X_{\tau_{\Omega}}$ has a density with respect to the Lebesgue measure on $\partial \Omega$ given by

$$
z \in \partial \Omega \mapsto-\frac{h}{2 \lambda_{h}} \frac{\partial_{h} u_{h}(z) e^{-\frac{2}{h} f(z)}}{\int_{\Omega} u_{h}(y) e^{-\frac{2}{h} f(y)} d y},
$$

where $u_{h}$ is the eigenfunction associated with the smallest eigenvalue $\lambda_{h}$ of $-L_{f, h}^{D,(0)}(\Omega)$ (see Proposition 4).

Here and in the following, $\partial_{n}=n \cdot \nabla$ stands for the normal derivative and $n$ is the unit outward normal on $\partial \Omega$.

This proposition shows that, starting from the quasi-stationary distribution in the domain $\Omega$, the exit event can be modeled by a jump Markov process without any approximation. Indeed, using the notation of Section 1.2 , let us consider that $\Omega \subset \mathbb{R}^{d}$ is associated with the state 0 . Let us assume that $\Omega$ is surrounded be $n$ neighbourding states, associated with domains $\left(\Omega_{i}\right)_{i=1, \ldots, n}$ (see Figure 1 for a schematic representation when $n=4)$. Let us define the transition rates:

$$
\forall i \in\{1, \ldots n\}, k_{0, i}=\frac{\mathbb{P}_{\nu_{h}}\left(X_{\tau_{\Omega}} \in \partial \Omega \cap \Omega_{i}\right)}{\mathbb{E}_{\nu_{h}}\left(\tau_{\Omega}\right)} .
$$

Then, by Proposition 6, the exit event is such that:

- The residence time $\tau_{\Omega}$ is exponentially distributed with parameters $\sum_{i=1}^{n} k_{0, i}$.

- The next visited state is independent of the residence time and is $i$ with probability $\frac{k_{0, i}}{\sum_{j=1}^{n} k_{0, j}}$.

This is exactly the two properties (4) and (5) which are required to define a transition using a jump Markov process. The quasi stationary distribution can thus be used to parameterize the underlying jump Markov process if the domains are metastable.

The question we would like to address in this work is now the following: what is the error introduced when one approximates the exact rates (15) using the Eyring-Kramers formula (6)-(7). From Proposition 6 , since $\mathbb{E}_{\nu_{h}}\left(\tau_{\Omega}\right)=1 / \lambda_{h}$, one has the following formula for the exact rates:

$$
k_{0, i}=-\frac{h}{2} \frac{\int_{\partial \Omega \cap \partial \Omega_{i}}\left(\partial_{n} u_{h}\right)(z) e^{-\frac{2}{h} f(z)} \sigma(d z)}{\int_{\Omega} u_{h}(y) e^{-\frac{2}{h} f(y)} d y}
$$

where $\sigma$ denotes the Lebesgue measure on $\partial \Omega$. We will be able to prove that in the small temperature regime $h \rightarrow 0$, the exact rates (16) can indeed be accurately approximated by the Eyring-Kramers formula (6) with explicit error bounds. The asymptotic analysis is done directly on the rates, and not only on the logarithm of the rates (which is the typical result obtained with the large deviation theory for example, see Section 1.3). 


\subsection{Statement of the main result}

We state in this section the main result of this work (Theorem 1) on the asymptotic behavior of the normal derivative $\partial_{n} u_{h}$ in the regime $h \rightarrow 0$, as well as its corollary on the exit point density and the accuracy of the approximation of the exit rates by the Eyring-Kramers formula.

This section is organized as follows. We introduce in Section 1.5.1 a crucial tool in our analysis, the Agmon distance. Then, in Section 1.5.2, we give the set of hypotheses which will be needed throughout this work. Finally, Section 1.5 .3 is dedicated to the statement of our main result.

\subsubsection{Agmon distance}

Our results hold under some geometric assumptions which require to introduce the so-called Agmon distance. The objective of this section is to introduce this distance, which is particularly useful to quantify the decay of eigenfunctions away from critical points 40,75 . We introduce the Agmon distance in a general setting, namely for $\Omega$ a Riemannian manifold, but one could think of $\Omega$ as a $C^{\infty}$ connected open bounded subset of $\mathbb{R}^{d}$.

Definition 7. Let $\bar{\Omega}$ be a $C^{\infty}$ oriented connected compact Riemannian manifold of dimension d with boundary $\partial \Omega$ and $f: \bar{\Omega} \rightarrow \mathbb{R}$ be $C^{\infty}$. Define $g: \bar{\Omega} \rightarrow \mathbb{R}$ by

$$
\forall x \in \Omega, g(x)=|\nabla f(x)| \text { and } \forall x \in \partial \Omega, g(x)=\left|\nabla_{T} f(x)\right|,
$$

where for any $x \in \partial \Omega, \nabla_{T} f(x)$ denotes the tangential gradient of the function $f$ on $\partial \Omega$, i.e. $\nabla_{T} f(x)=\nabla f(x)-(\nabla f(x) \cdot n) n$, where $n$ is the unit outward normal to $\partial \Omega$ at $x$. One defines the length $L$ of a Lipschitz curve $\gamma: I \rightarrow \bar{\Omega}$, where $I \subset \mathbb{R}$ is an interval, by

$$
L(\gamma, I):=\int_{I} g(\gamma(t))\left|\gamma^{\prime}(t)\right| d t \in[0+\infty] .
$$

Let us recall that the Rademacher theorem (see for example [29]) states that every Lipschitz function admits almost everywhere a derivative (which is then bounded by the Lipschitz constant). Therefore, if $I$ is bounded, then $L(\gamma, I)<\infty$. Let us now define the Agmon distance.

Definition 8. Let $g$ be the function introduced in Definition 7 . The Agmon distance between $x \in \bar{\Omega}$ and $y \in \bar{\Omega}$ is defined by

$$
d_{a}(x, y)=\inf _{\gamma \in \operatorname{Lip}(x, y)} L(\gamma,(0,1))
$$

where $\operatorname{Lip}(x, y)$ is the set of curve $\gamma:[0,1] \rightarrow \bar{\Omega}$ which are Lipschitz with $\gamma(0)=x$, $\gamma(1)=y$.

The Agmon distance is obviously symmetric, non negative and satisfies the triangular inequality. It is a distance if the critical points of $f$ and $f_{\left.\right|_{\partial \Omega}}$ are isolated (see Proposition 42 below). Let us mention that in the case when $\Omega$ is a manifold without boundary, the Agmon distance introduced in Definition 8 coincides with the Agmon distance defined in [43, Appendix 2]. 
We will give in Section 3 more details about the Agmon distance we consider. In particular, it will be shown that the Agmon distance to the critical points of $\left.f\right|_{\partial \Omega}$ coincides with the solution to the eikonal equation $|\nabla \Phi|^{2}=|\nabla f|^{2}$ in neighborhoods of the critical points. This requires to use the tangential gradient of $f$ on $\partial \Omega$ in the definition of the Agmon distance (see (17)).

\subsubsection{Notations and hypotheses}

As already stated above, we assume that $\Omega$ is a connected open bounded $C^{\infty}$ domain of $\mathbb{R}^{d}$ and $f: \bar{\Omega} \rightarrow \mathbb{R}$ is a $C^{\infty}$ function 11 We will need the following set of assumptions:

[H1] The function $f: \bar{\Omega} \rightarrow \mathbb{R}$ is a Morse function on $\Omega$ and the restriction of $f$ to the boundary of $\Omega$ denoted by $\left.f\right|_{\partial \Omega}$, is a Morse function. The function $f$ does not have any critical point on $\partial \Omega$.

[H2] The function $f$ has a unique global minimum $x_{0} \in \Omega$ in $\bar{\Omega}$ :

$$
\min _{\partial \Omega} f>\min _{\bar{\Omega}} f=\min _{\Omega} f=f\left(x_{0}\right) .
$$

The point $x_{0}$ is the unique critical point of $f$ in $\bar{\Omega}$. The function $\left.f\right|_{\partial \Omega}$ has exactly $n \geq 1$ local minima denoted by $\left(z_{i}\right)_{i=1, \ldots, n}$ such that $f\left(z_{1}\right) \leq f\left(z_{2}\right) \leq \ldots \leq f\left(z_{n}\right)$.

[H3] $\partial_{n} f>0$ on $\partial \Omega$.

In the following, $n_{0} \in\{1, \ldots, n\}$ denotes the number of points in $\left.\arg \min f\right|_{\partial \Omega}$ :

$$
f\left(z_{1}\right)=\ldots=f\left(z_{n_{0}}\right)<f\left(z_{n_{0}+1}\right) \leq \ldots \leq f\left(z_{n}\right) .
$$

We will need to define the basins of attraction of the local minima $z_{i}$ for the dynamics $\dot{x}=-\nabla_{T} f(x)$ in $\partial \Omega$, where, we recall, for any $x \in \partial \Omega, \nabla_{T} f(x)$ denotes the tangential gradient of $f$ on $\partial \Omega$.

Definition 9. Assume that [H1] holds. For each local minimum $z \in \partial \Omega$, one denotes by $B_{z} \subset \partial \Omega$ the basin of attraction of $z$ for the dynamics in $\partial \Omega \dot{x}=-\nabla_{T} f(x)$ : denoting by $\varphi_{t}(y)$ the solution to $\frac{d}{d t} \varphi_{t}(y)=-\nabla_{T} f\left(\varphi_{t}(y)\right)$ with initial condition $\varphi_{0}(y)=y \in \partial \Omega$, one has $B_{z}:=\left\{y \in \partial \Omega, \lim _{t \rightarrow \infty} \varphi_{t}(y)=z\right\}$. Notice that $B_{z}$ is an open subset of $\partial \Omega$. Additionally, one defines $B_{z}^{c}:=\partial \Omega \backslash B_{z}$.

From this definition, one obviously has that for each local minimum $z \in \partial \Omega$, for any $x \in B_{z}, f(x) \geq f(z)$. On Figure 2, one gives a schematic representation in dimension 2 of a function $f$ satisfying the assumptions [H1], [H2], and [H3], and of its restriction to $\partial \Omega$, in the case $n=4$ and $n_{0}=2$.

As a consequence of the assumption [H1], the determinants of the Hessians of $f$ (resp. of $\left.f\right|_{\partial \Omega}$ ) at the critical points of $f$ (resp. of $\left.f\right|_{\partial \Omega}$ ) are non zero. These quantities appear in the prefactors of the Eyring-Kramers law (see Equation 25 below).

\footnotetext{
${ }^{1}$ Actually, as explained in Section 2 we will perform the analysis in a more general setting, namely when $\bar{\Omega}$ is a $C^{\infty}$ oriented connected compact Riemannian manifold. In this introductory section, we stick to a simpler presentation, with $\Omega$ a subset of $\mathbb{R}^{d}$.
} 

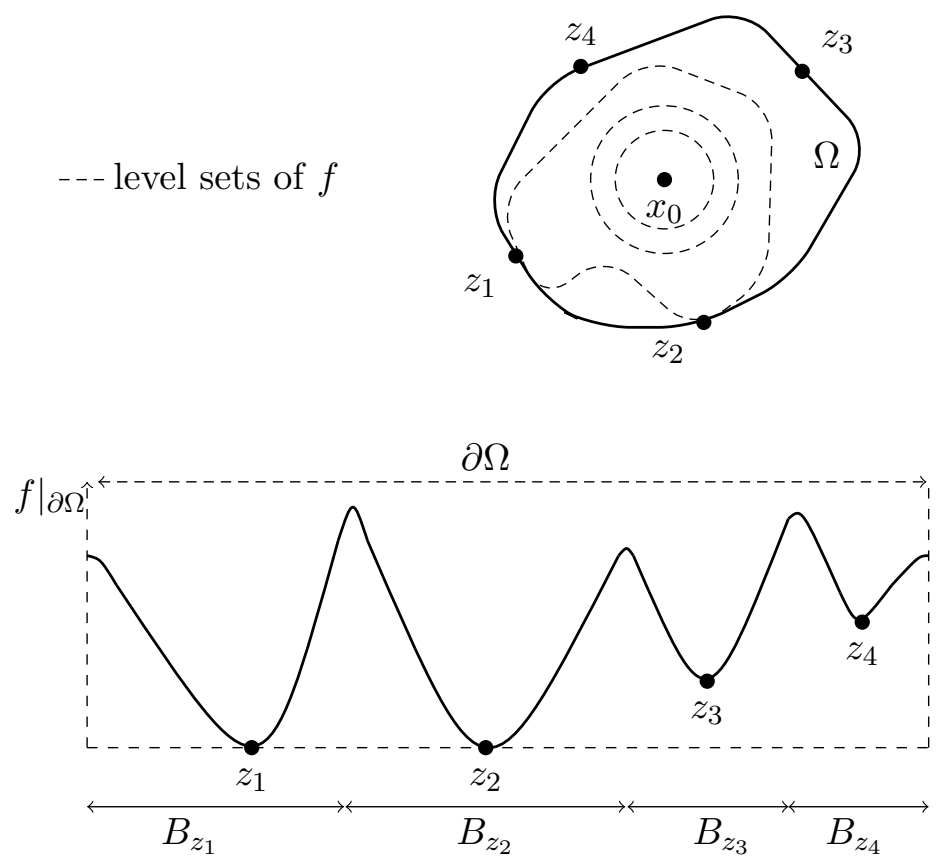

Figure 2: Schematic representation in dimension 2 of a function $f$ satisfying the assumptions [H1], [H2], and [H3], and of its restriction $\left.f\right|_{\partial \Omega}$ to $\partial \Omega$. On the figure, $n=4$ and $n_{0}=2$.

Remark 10. Let us recall how the Hessians are defined. Let $\phi: N \rightarrow \mathbb{R}$ be a $C^{\infty}$ function defined on a Riemannian $C^{\infty}$ manifold $N$ of dimension d. By standard results of Riemannian geometry, the Hessian Hess $\phi(x)$ of $\phi$ at a point $x \in N$ is defined as a bilinear symmetric form acting on vectors in the tangent space $T_{x} N$ as:

$$
\forall X, Y \in \Gamma(T N), \operatorname{Hess} \phi(X, Y)=\nabla_{X} d \phi(Y)
$$

where $\nabla$ is the covariant derivative (Levi-Civita connection) and $d \phi$ is the differential of $\phi$. Then, detHess $\phi(x)$ is defined as the determinant of the bilinear form Hess $\phi(x)$ in any orthonormal basis of $T_{x} N$.

In practice, det Hess $\phi(x)$ can be computed at a critical point of $\phi$ using a local chart as follows. Let us assume that $x_{0}$ is a critical point of $\phi: d_{x_{0}} \phi=0$. Let us introduce $\psi: y \in U \mapsto \psi(y) \in V$ a local chart around $x_{0}$, where $U \subset \mathbb{R}^{d}$ is a neighborhood of $0, V \subset N$ is a neighborhood of $x_{0}$ and $\psi(0)=x_{0}$. Let us assume in addition that the vectors $\left(e_{i}\right)_{i=1, \ldots, d}:=\left(\frac{\partial \psi}{\partial y_{i}}(0)\right)_{i=1, \ldots, d}$ are orthonormal (thus defining an orthonormal basis of $\left.T_{x_{0}} N\right)$. Let us introduce the symmetric matrix $H$ associated with the second order differential of $\phi \circ \psi$ at point $0: \forall(u, v) \in \mathbb{R}^{d} \times \mathbb{R}^{d}$

$$
D_{0}^{2}(\phi \circ \psi)\left(\sum_{i=1}^{d} u_{i} e_{i}, \sum_{i=1}^{d} v_{i} e_{i}\right)=u^{T} H v .
$$

Then

$$
\operatorname{det} \operatorname{Hess} \phi\left(x_{0}\right)=\operatorname{det} H .
$$

This formula is only valid at a critical point and is a direct consequence of the definition (19) of the Hessian and the explicit expression of the Levi Civita connection in the 
local chart $\psi$ :

$$
\left.\nabla_{X} d \phi(Y)\right|_{x}=\sum_{i, j=1}^{d}\left(\frac{\partial^{2}(\phi \circ \psi)}{\partial y_{i} \partial y_{j}}(y)-\sum_{k=1}^{d} \Gamma_{i, j}^{k}(\psi(y)) \frac{\partial(\phi \circ \psi)}{\partial y_{k}}(y)\right) Y_{i} X_{j}
$$

where $x=\psi(y) \in V, \Gamma_{i, j}^{k}(x)$ are the Christoffel symbols of the connection $\nabla$ associated with the basis $\left(\left(\partial_{y_{j}} \psi\right)\left(\psi^{-1}(x)\right)_{j=1, \ldots, n}\right.$ of $T_{x} N$ and $\left(X_{j}\right)_{j=1, \ldots, n}$ (respectively $\left(Y_{j}\right)_{j=1, \ldots, n}$ ) are the coordinates of $X$ (respectively $Y$ ) in this basis.

\subsubsection{Main result}

In view of equations (14) and (16), we need to give an estimate of three quantities in order to analyze the exit point density and the asymptotic of the transition rates in the regime $h \rightarrow 0: \int_{\Sigma}\left(\partial_{n} u_{h}\right) e^{-\frac{2}{h} f}$ for a subset $\Sigma$ of $\partial \Omega, \int_{\Omega} u_{h} e^{-\frac{2}{h} f}$ and $\lambda_{h}$, where, we recall $\left(\lambda_{h}, u_{h}\right)$ is defined by (12). We will consider a subset $\Sigma$ such that $\Sigma \subset B_{z_{i}}$ for a local minimum $z_{i}$ (see Definition 9 for the definition of $B_{z_{i}}$ ).

Theorem 1. Assume that [H1], [H2] and [H3] hold. Moreover assume that

- $\forall i \in\{1, \ldots, n\}$,

$$
\inf _{z \in B_{z_{i}}^{c}} d_{a}\left(z, z_{i}\right)>\max \left[f\left(z_{n}\right)-f\left(z_{i}\right), f\left(z_{i}\right)-f\left(z_{1}\right)\right],
$$

- and

$$
f\left(z_{1}\right)-f\left(x_{0}\right)>f\left(z_{n}\right)-f\left(z_{1}\right) .
$$

Then, for all $i \in\{1, \ldots, n\}$ and all open set $\Sigma_{i} \subset \partial \Omega$ containing $z_{i}$ and such that $\bar{\Sigma}_{i} \subset B_{z_{i}}$, in the limit $h \rightarrow 0$

$$
\int_{\Sigma_{i}}\left(\partial_{n} u_{h}\right) e^{-\frac{2}{h} f} d \sigma=A_{i}(h) e^{-\frac{2 f\left(z_{i}\right)-f\left(x_{0}\right)}{h}}(1+O(h)),
$$

where $u_{h}$ is the eigenfunction associated with the smallest eigenvalue of $-L_{f, h}^{D,(0)}(\Omega)$ (see Proposition 4) which satisfies (11) and

$$
A_{i}(h)=-\frac{\left(\operatorname{det} \operatorname{Hess} f\left(x_{0}\right)\right)^{1 / 4} \partial_{n} f\left(z_{i}\right) 2 \pi^{\frac{d-2}{4}}}{\sqrt{\left.\operatorname{det} \operatorname{Hess} f\right|_{\partial \Omega}\left(z_{i}\right)}} h^{\frac{d-6}{4}} .
$$

Let us mention that the importance of the geometric assumptions (20) and (21) will be discussed in Section 1.6.2

Remark 11. As will become clear in the proof of Theorem 1, it can actually be proven that for all $i \in\{1, \ldots, n\}$, the residual $r_{i}(h)=O(h)$ appearing in 22 admits a full asymptotic expansion in $h$ : there exists a sequence $\left(b_{k, i}\right)_{k \geq 0} \in \mathbb{R}^{\mathbb{N}}$ such that for all $N \in \mathbb{N}$, in the limit $h \rightarrow 0$,

$$
r_{i}(h)=h \sum_{k=0}^{N} b_{k, i} h^{k}+O\left(h^{N+2}\right) .
$$

We do not state our main result with this expansion since, for general domains $\Omega$, the explicit computations of the sequence $\left(b_{k, i}\right)_{k \geq 0}$ is not possible in practice. This remark also holds for all the residuals $O(h)$ in the next results. 
Proposition 12. Assume that [H1], [H2] and [H3] hold. Then when $h \rightarrow 0$

$$
\int_{\Omega} u_{h}(x) e^{-\frac{2}{h} f(x)} d x=\frac{\pi^{\frac{d}{4}}}{\left(\operatorname{det} \operatorname{Hess} f\left(x_{0}\right)\right)^{1 / 4}} h^{\frac{d}{4}} e^{-\frac{1}{h} f\left(x_{0}\right)}(1+O(h)),
$$

where $u_{h}$ is the eigenfunction associated with the smallest eigenvalue of $-L_{f, h}^{D,(0)}(\Omega)($ see Proposition 4) which satisfies (11).

Proposition 13. Assume that [H1], [H2], and [H3] hold. Then, in the limit $h \rightarrow 0$,

$$
\lambda_{h}=\frac{\sqrt{\operatorname{det} \operatorname{Hess} f\left(x_{0}\right)}}{\sqrt{\pi h}} \sum_{i=1}^{n_{0}} \frac{\partial_{n} f\left(z_{i}\right)}{\sqrt{\left.\operatorname{det} \operatorname{Hess} f\right|_{\partial \Omega}\left(z_{i}\right)}} e^{-\frac{2}{h}\left(f\left(z_{1}\right)-f\left(x_{0}\right)\right)}(1+O(h)),
$$

where $\lambda_{h}$ is the smallest eigenvalue of $-L_{f, h}^{D,(0)}(\Omega)$ (see Proposition 4).

Theorem 1 is the main contribution of this work. Actually Theorem 1 will be proven in a more general framework: namely when $\bar{\Omega}$ is a $C^{\infty}$ connected compact oriented Riemannian $d$-dimensional manifold with boundary $\partial \Omega$. Theorem 1, Proposition 12 and Proposition 13 are respectively proved in Sections 4.5, 5.1.1 and 5.1.2. For the sake of completeness, we provide a proof of Proposition 13 in our specific setting, but this result actually holds under weaker geometric assumptions, see [24] or [39].

These results have the following consequence on the first exit point distribution and the estimate of the exact rates $\left(k_{0, i}\right)_{i=1, \ldots, n}$ using the Eyring-Kramers formula (see Section 1.4.3). We recall that $\left(X_{t}\right)_{t \geq 0}$ denotes the solution to (1), $\tau_{\Omega}$ is the exit time from the domain $\Omega$ and $\nu_{h}$ is the quasi stationary distribution associated with $\left(X_{t}\right)_{t \geq 0}$ and $\Omega$.

Corollary 14. Under the hypotheses of Theorem 1, for $i \in\{1, \ldots, n\}$ and for all open sets $\Sigma_{i} \subset \partial \Omega$ containing $z_{i}$ and such that $\bar{\Sigma}_{i} \subset B_{z_{i}}$, in the limit $h \rightarrow 0$ :

$$
\begin{aligned}
\mathbb{P}_{\nu_{h}}\left[X_{\tau_{\Omega}} \in \Sigma_{i}\right]= & \frac{\partial_{n} f\left(z_{i}\right)}{\sqrt{\operatorname{det} \operatorname{Hess} f_{\mid \partial \Omega}\left(z_{i}\right)}}\left(\sum_{k=1}^{n_{0}} \frac{\partial_{n} f\left(z_{k}\right)}{\sqrt{\operatorname{det} \operatorname{Hess} f_{\mid \partial \Omega}\left(z_{k}\right)}}\right)^{-1} \\
& \times e^{-\frac{2}{h}\left(f\left(z_{i}\right)-f\left(z_{1}\right)\right)}(1+O(h)) .
\end{aligned}
$$

As a simple consequence of Corollary 14 , we obtain the expected result that $\left(X_{t}\right)_{t \geq 0}$ leaves $\Omega$ around the global minima of $f$ on $\partial \Omega$ : for any collection of open sets $\left(\Sigma_{j}\right)_{1 \leq j \leq n_{0}}$ such that for all $j \in\left\{1, \ldots, n_{0}\right\}, \bar{\Sigma}_{j} \subset B_{z_{j}}$ and $z_{j} \in \Sigma_{j}$, in the limit $h \rightarrow 0$, $\mathbb{P}_{\nu_{h}}\left[X_{\tau_{\Omega}} \in \bigcup_{j=1}^{n_{0}} \Sigma_{j}\right]=1+O(h)$. Actually, this latter result can be proven with an exponentially small residual $\left(O(h)\right.$ is replaced by $O\left(e^{-c / h}\right)$ for some positive $\left.c\right)$ in a more general setting (see for instance $[18,19,31,47,68,77]$ ). Let us also refer to [24] where we discuss this result in a more general setting (for example $f$ can have several critical points in $\Omega$ and the assumptions (20) and (21) are not needed).

Corollary 15. Let us assume that the hypotheses of Theorem 1 are satisfied. Let $i \in\{1, \ldots, n\}$ and $\Sigma_{i} \subset \partial \Omega$ be an open set containing $z_{i}$ such that $\bar{\Sigma}_{i} \subset B_{z_{i}}$. Using the notation of Section 1.4.3, assume that $\Sigma_{i}$ is the common boundary between $\Omega$ and another domain $\Omega_{i} \subset \mathbb{R}^{d}$. Under the hypotheses of Theorem 1 , the transition rate given by (15), to go from $\Omega$ to $\Omega_{i}$ satisfies, in the limit $h \rightarrow 0$,

$$
k_{0, i}=\frac{1}{\sqrt{\pi h}} \partial_{n} f\left(z_{i}\right) \frac{\sqrt{\operatorname{det} \operatorname{Hess} f\left(x_{0}\right)}}{\sqrt{\operatorname{det} \operatorname{Hess} f_{\mid \partial \Omega}\left(z_{i}\right)}} e^{-\frac{2}{h}\left(f\left(z_{i}\right)-f\left(x_{0}\right)\right)}(1+O(h)) .
$$


This corollary thus gives a justification of the Eyring-Kramers formula and the Transition State Theory to build Markov models. As stated in the assumptions, the exit rates are obtained assuming $\partial_{n} f>0$ on $\partial \Omega$ : the local minima $z_{1}, \ldots, z_{n}$ of $f$ on $\partial \Omega$ are therefore not saddle points of $f$ but so-called generalized saddle points (see [39, 54]). This appellation "generalized saddle points" is justified by the fact that, under [H1], [H2], [H3] and when $f$ is extended by $-\infty$ outside $\bar{\Omega}$ (which is consistent with the Dirichlet boundary conditions used to define $\left.L_{f, h}^{D,(0)}\right)$, the points $\left(z_{i}\right)_{i=1, \ldots, n}$ are geometrically saddle points of $f: z_{i}$ is a local minimum of $\left.f\right|_{\partial \Omega}$ and a local maximum of $\left.f\right|_{D_{i}}$, where $D_{i}$ is the straight line passing through $z_{i}$ and orthogonal to $\partial \Omega$ at $z_{i}$. In a future work, we intend to extend these results to the case where the points $\left(z_{i}\right)_{1 \leq i \leq n}$ are saddle points of $f$, in which case we expect to prove the same result 25 for the exit rates, with a modified prefactor:

$$
A_{0, i}=\frac{1}{\pi}\left|\lambda^{-}\left(z_{i}\right)\right| \frac{\sqrt{\operatorname{det} \operatorname{Hess} f\left(x_{0}\right)}}{\sqrt{\left|\operatorname{det} \operatorname{Hess} f\left(z_{i}\right)\right|}}
$$

(this formula can be obtained using formal expansions on the exit time and Laplace's method). Notice that the latter formula differs from (6)-(7) by a multiplicative factor $1 / 2$ since $\lambda_{h}$ is the exit rate from $\Omega$ and not the transition rate to one of the neighboring state. Concerning this multiplicative factor $1 / 2$, we refer for example to the remark on page 408 in [5], 55, Remark 10], and the results on asymptotic exit times in [58]. This factor is due to the fact that once on the saddle point, the process has a probability one half to go back to $\Omega$, and a probability one half to effectively leave $\Omega$, in the limit $h \rightarrow 0$. This multiplicative factor does not have any influence on the law of the next visited state which only involves ratio of the rates $k_{0, i}$, see Section 1.4.3 and Equation (24).

\subsection{Discussion and generalizations}

As explained above, the interest of Theorem 1 is that it justifies the use of the EyringKramers formula to model the exit event using a jump Markov model including the prefactors. It gives in particular the relative probability to leave $\Omega$ through each of the local minima $z_{i}$ of $f$ on the boundary $\partial \Omega$. Moreover, one obtains an estimate of the relative error on the exit probabilities (and not only on the logarithm of the exit probabilities as in (8)): it is of order $h$, see Equation (24).

In Section 1.6.1, we explain how this result can be generalized to a situation where the process $\left(X_{t}\right)_{t \geq 0}$ is assumed to start under another initial condition than the quasi stationary distribution. The importance of the geometric assumption 20)-21] (resp. assumption (26) to obtain the asymptotic result of Corollary 14 (resp. its generalization to deterministic initial conditions, see Corollary 16) is discussed in Section 1.6.2. Finally, in Section 1.6.3. we discuss extensions to less stringent conditions than 20(21). Moreover the exit through subsets of $\partial \Omega$ which do not necessarily contain one of the local minima $z_{i}$ of $\left.f\right|_{\partial \Omega}$ is considered: this shows in particular the interest of estimating the prefactors in the asymptotic approximations of the exit rates.

\subsubsection{Extension of the result to other initial conditions}

The question we would like to address in this section is how to generalize Corollary 14. to a deterministic initial condition: $X_{0}=x$ for $x \in \Omega$. 
Corollary 16. Let us assume that all the hypotheses of Corollary 14 are satisfied, and that in addition there exists $i_{0} \in\{2, \ldots, n\}$ such that

$$
2\left(f\left(z_{i_{0}}\right)-f\left(z_{1}\right)\right)<f\left(z_{1}\right)-f\left(x_{0}\right) .
$$

Let $j \in\left\{1, \ldots, i_{0}\right\}$ and $\alpha \in \mathbb{R}$ be such that

$$
f\left(x_{0}\right)<\alpha<2 f\left(z_{1}\right)-f\left(z_{j}\right) .
$$

Then, for $i \in\{1, \ldots, j\}$ and for all open sets $\Sigma_{i} \subset \partial \Omega$ containing $z_{i}$ and such that $\bar{\Sigma}_{i} \subset B_{z_{i}}$, we have uniformly in $x \in f^{-1}((-\infty, \alpha]) \cap \Omega$, in the limit $h \rightarrow 0$ :

$\mathbb{P}_{x}\left[X_{\tau_{\Omega}} \in \Sigma_{i}\right]=\frac{\partial_{n} f\left(z_{i}\right)}{\sqrt{\operatorname{det} \operatorname{Hess} f_{\mid \partial \Omega}\left(z_{i}\right)}}\left(\sum_{k=1}^{n_{0}} \frac{\partial_{n} f\left(z_{k}\right)}{\sqrt{\operatorname{det} \operatorname{Hess} f_{\mid \partial \Omega}\left(z_{k}\right)}}\right)^{-1} e^{-\frac{2}{h}\left(f\left(z_{i}\right)-f\left(z_{1}\right)\right)}(1+O(h))$.

Let us give a simple example to illustrate this result. In a situation where $n=2$, this corollary shows that the estimates we have obtained on the probability to exit in a neighborhood of $z_{2}$ under the assumption $X_{0} \sim \nu_{h}$ are still valid if $X_{0}=x$ for $x \in$ $f^{-1}\left(\left(-\infty, 2 f\left(z_{1}\right)-f\left(z_{2}\right)\right)\right) \cap \Omega$ under the assumption $f\left(z_{1}\right)-f\left(x_{0}\right)>2\left(f\left(z_{2}\right)-f\left(z_{1}\right)\right)$, which is a stronger assumption than (21).

\subsubsection{On the geometric assumptions (20), 21) and (26)}

On the geometric assumption 20).

The question we would like to address is the following: is the assumption (20) necessary for the result on the exit point density (24) to hold?

In order to test this assumption numerically, we consider the following simple twodimensional setting. The potential function is

$$
f(x, y)=x^{2}+y^{2}-a x,
$$

with $a \in(0,1 / 9)$, and the domain $\Omega$ is defined by (see Figure 3):

$$
\Omega=(-1,1)^{2} \cup\left\{(x, y) \mid x^{2}+(y-1)^{2}<1\right\} \cup\left\{(x, y) \mid x^{2}+(y+1)^{2}<1\right\} .
$$

The two local minima of $f$ on $\partial \Omega$ are $z_{1}=(1,0)$ and $z_{2}=(-1,0)$. Notice that $f\left(z_{2}\right)-$ $f\left(z_{1}\right)=2 a>0$. The potential $f$ has a unique critical point in $\Omega$, namely the global minimum $x_{0}=(a / 2,0)$. Let us check that the assumptions of Theorem 1 are satisfied in this setting (i.e. for $\left.a \in\left(0, \frac{1}{9}\right)\right)$. Indeed, the inequality $f\left(z_{1}\right)-f\left(x_{0}\right)>f\left(z_{2}\right)-f\left(z_{1}\right)$ is satisfied if and only if $1-3 a+\frac{a^{2}}{4}>0$ i.e. if and only if $a \notin(2(3-\sqrt{8}), 2(3+\sqrt{8}))$. Moreover, using Proposition 46, the inequality $d_{a}\left(z_{1}, B_{z_{1}}^{c}\right)>f\left(z_{2}\right)-f\left(z_{1}\right)$ is satisfied. Finally, to check that the inequality $d_{a}\left(z_{2}, B_{z_{2}}^{c}\right)>f\left(z_{2}\right)-f\left(z_{1}\right)$ is satisfied we use Proposition 45 with $W=\left\{(x, y) \in \mathbb{R}^{2},\left|(x, y)-z_{2}\right| \leq \frac{1}{3}\right\} \cap \bar{\Omega}$ and $W^{\prime}=\{(x, y) \in$ $\left.\mathbb{R}^{2},\left|(x, y)-z_{2}\right| \leq \frac{2}{3}\right\} \cap \bar{\Omega}$. In that case, one has $\alpha=\frac{1}{3}$ (where $\alpha$ is defined by (87)) and thus the inequality

$$
\alpha \inf _{x \in W^{\prime} \backslash W} g(x)=\frac{1}{3} \min \left(\frac{2}{3},\left|2\left(-1+\frac{2}{3}\right)-a\right|\right)=\frac{1}{3} \min \left(\frac{2}{3}, \frac{2}{3}+a\right)>f\left(z_{2}\right)-f\left(z_{1}\right)=2 a
$$




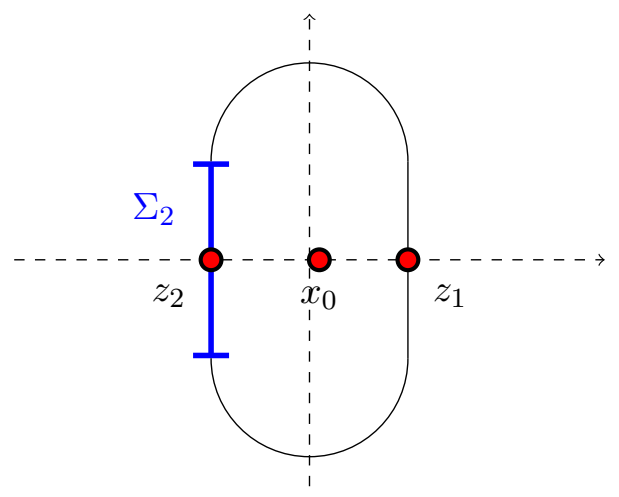

Figure 3: The domain $\Omega$.

is satisfied if and only if $a<\frac{1}{9}$.

Let us consider the segment $\Sigma_{2}$ joining the two points $(-1,-1)$ and $(-1,1)$. This subset of $\partial \Omega$ contains the highest saddle point $z_{2}$ and is included in $B_{z_{2}}$. From Theorem 1, we expect that, in the limit $h \rightarrow 0$,

$$
\mathbb{P}_{\nu_{h}}\left[X_{\tau_{\Omega}} \in \Sigma_{2}\right]=\exp \left(G\left(\frac{2}{h}\right)\right)(1+O(h))
$$

where

$$
G(x)=\ln \left[\frac{\partial_{n} f\left(z_{2}\right) \sqrt{\left.\operatorname{det} \operatorname{Hess} f\right|_{\partial \Omega}\left(z_{1}\right)}}{\partial_{n} f\left(z_{1}\right) \sqrt{\left.\operatorname{det} \operatorname{Hess} f\right|_{\partial \Omega}\left(z_{2}\right)}}\right]-x\left(f\left(z_{2}\right)-f\left(z_{1}\right)\right) .
$$

The function $G$ is compared for various value sod $h$ to the numerically estimated function $F$ defined by $F\left(\frac{2}{h}\right)=\ln \left(\mathbb{P}_{\nu_{h}}\left[X_{\tau_{\Omega}} \in \Sigma_{2}\right]\right)$. In practice, the quasi stationary distribution $\nu_{h}$ is sampled using a Fleming-Viot particle system (the convergence diagnostics is based on a Gelman-Rubin statistics, see [3]) composed of $10^{5}$ particles. The probability $\mathbb{P}_{\nu_{h}}\left(X_{\tau_{\Omega}} \in \Sigma_{2}\right)$ is estimated using a Monte Carlo procedure using $6 \times 10^{5}$ particles distributed according to the quasi stationary distribution $\nu_{h}$. The dynamics (1) is discretized in time using an Euler-Maruyama scheme with a timestep $\Delta t$ which is made precise in the captions of the figures. On Figures 4 and 5, we observe an excellent agreement between the theory and the numerical results.

Now, the potential function $f$ is modified such that the assumption $(20)$ is not satisfied anymore. More precisely, the potential function is

$$
f(x, y)=\left(y^{2}-2 a(x)\right)^{3},
$$

with $a(x)=a_{1} x^{2}+b_{1} x+0.5$ where $a_{1}$ and $b_{1}$ are chosen such that $a(-1+\delta)=0$, $a(1)=1 / 4$ for $\delta=0.05$. We have $f\left(z_{1}\right)=-1 / 8$ and $f\left(z_{2}\right)=-8(a(-1))^{3}>0>f\left(z_{1}\right)$. Moreover, two 'corniches' (which are in the level set $f^{-1}(\{0\})$ of $f$, and on which $|\nabla f|=$ 0 ) on the 'slopes of the hills' of the potential $f$ join the point $(-1+\delta, 0)$ to $B_{z_{2}}^{c}$ (at the points $(1,-1 / \sqrt{2}) \in B_{z_{2}}^{c}$ and $\left.(1,1 / \sqrt{2}) \in B_{z_{2}}^{c}\right)$ so that $\inf _{z \in B_{z_{2}}^{c}} d_{a}\left(z, z_{2}\right)<f\left(z_{2}\right)-f\left(z_{1}\right)$. 


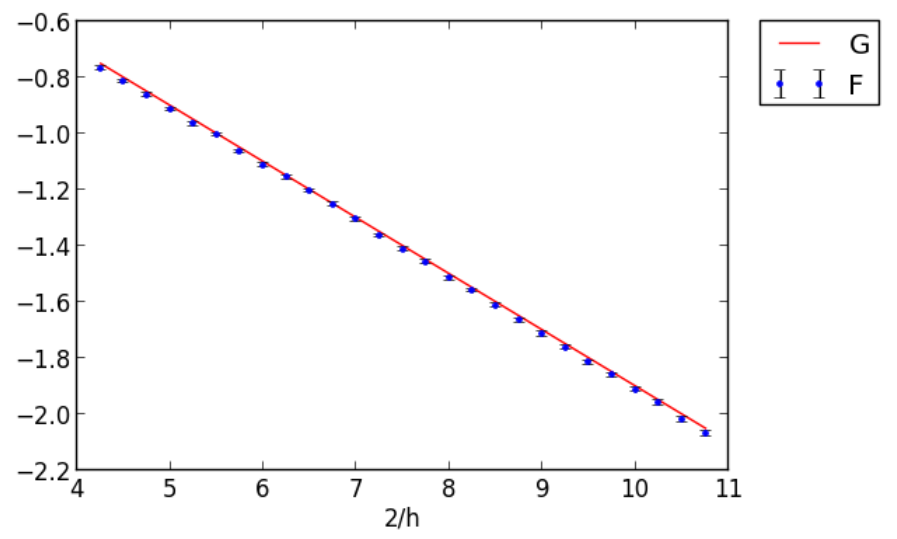

Figure 4: Logarithm of the probability $\mathbb{P}_{\nu_{h}}\left(X_{\tau_{\Omega}} \in \Sigma_{2}\right)$ as a function of $\frac{2}{h}$ : comparison of the theoretical result function $(G)$ with the numerical result (function $F, \Delta t=5.10^{-3}$ ); $a=1 / 10$.

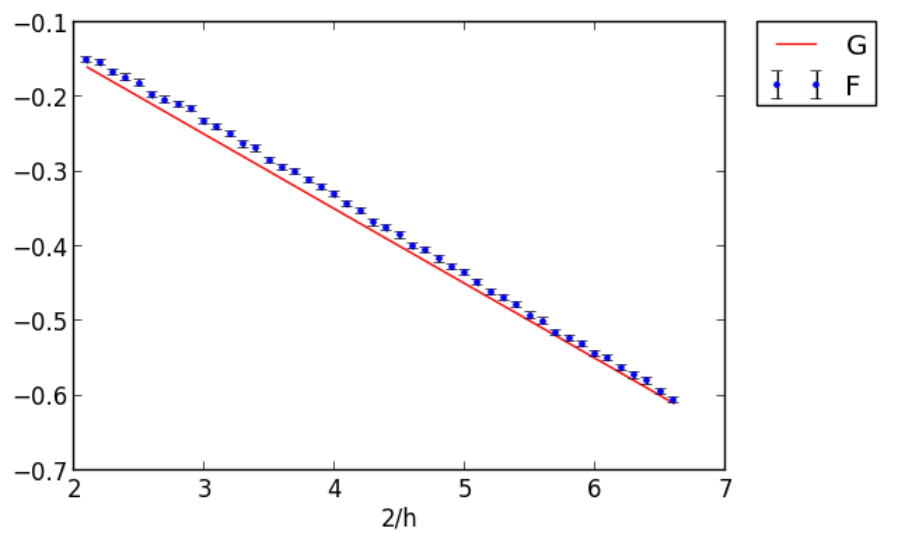

Figure 5: Logarithm of the probability $\mathbb{P}_{\nu_{h}}\left(X_{\tau_{\Omega}} \in \Sigma_{2}\right)$ as function of $\frac{2}{h}$ as a function of $\frac{2}{h}$ : comparison of the theoretical result function $(G)$ with the numerical result (function $\left.F, \Delta t=2.10^{-3}\right) ; a=1 / 20$.

Indeed, in that case assumption 20 is not satisfied since

$$
\begin{aligned}
\inf _{z \in B_{z_{2}}^{c}} d_{a}\left(z, z_{2}\right) & \leq d_{a}\left(z_{2},(1,1 / \sqrt{2})\right) \\
& \leq d_{a}\left(z_{2},(0,-1+\delta)\right)+d_{a}((0,-1+\delta),(1,1 / \sqrt{2})) \\
& =f\left(z_{2}\right)-f(0,-1+\delta)+0 \\
& =f\left(z_{2}\right)<f\left(z_{2}\right)-f\left(z_{1}\right) .
\end{aligned}
$$

Notice that the Hessians (Hess $\left.\left.f\right|_{\partial \Omega}\right)\left(z_{1}\right)$ and (Hess $\left.\left.f\right|_{\partial \Omega}\right)\left(z_{2}\right)$ are nonsingular. The functions $f_{\mid \Omega}$ and $\left.f\right|_{\partial \Omega}$ are not Morse functions, but an arbitrarily small perturbation (which we neglect here) turns them into Morse functions. When comparing the numerically estimated probability $\mathbb{P}_{\nu_{h}}\left(X_{\tau_{\Omega}} \in \Sigma_{2}\right)$, with the theoretical asymptotic result in the limit $h \rightarrow 0$, we observe a discrepancy on the prefactors, see Figure 6 .

Therefore, it seems that assumption $(20)$ is indeed required to get an accurate description of the dynamics by the jump Markov process using the Eyring-Kramers law to estimate the rates between the neighboring states. 


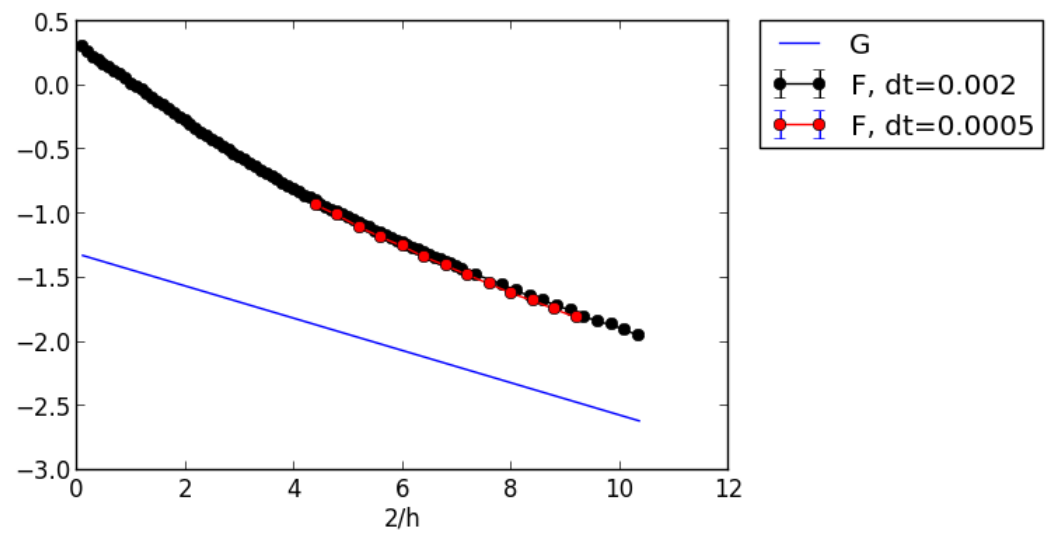

Figure 6: Logarithm of the probability $\mathbb{P}_{\nu_{h}}\left(X_{\tau_{\Omega}} \in \Sigma_{2}\right)$ as a function of $\frac{2}{h}$ : comparison of the theoretical result function $(G)$ with the numerical result (function $F, \Delta t=2.10^{-3}$ and $\left.\Delta t=5 \cdot 10^{-4}\right)$.

On the geometric assumptions (21) and (26).

To discuss the necessity of the assumptions (26) in Corollary 16 and (21) in Corollary 14 , we consider a one-dimensional case, where the law of $X_{\tau_{\Omega}}$ when $X_{0}=x$ has an explicit expression. Let $f: \mathbb{R} \rightarrow \mathbb{R}$ be $C^{\infty}$ and let $z_{1}, z_{2} \in \mathbb{R}$ such that $z_{1}<z_{2}$. Let us assume that $f^{\prime}\left(z_{1}\right)<0, f^{\prime}\left(z_{2}\right)>0, f\left(z_{1}\right)<f\left(z_{2}\right)$ and $f$ has only one critical point in $\left(z_{1}, z_{2}\right)$ denoted by $x_{0}$. This implies in particular that $f\left(x_{0}\right)=\min _{\left[z_{1}, z_{2}\right]} f<f\left(z_{1}\right)$. Moreover let us assume that $f^{\prime \prime}\left(x_{0}\right)>0$. Therefore, the hypotheses [H1]-[H2]-[H3] hold. For $x \in\left[z_{1}, z_{2}\right]$, let us denote by $w_{h}(x)=\mathbb{P}_{x}\left[X_{\tau_{\left(z_{1}, z_{2}\right)}}=z_{2}\right]$. It is standard that using a Feynman-Kac formula, $w_{h}$ solves the elliptic boundary value poblem

$$
\frac{h}{2} w_{h}^{\prime \prime}-w_{h}^{\prime} f^{\prime}=0, w_{h}\left(z_{1}\right)=0, w_{h}\left(z_{2}\right)=1 .
$$

Therefore, one has for $x \in\left[z_{1}, z_{2}\right]$ :

$$
w_{h}(x)=\frac{\int_{z_{1}}^{x} e^{\frac{2}{h} f}}{\int_{z_{1}}^{z_{2}} e^{\frac{2}{h} f}} .
$$

Let $x \in\left[z_{1}, z_{2}\right]$. Using Laplace's method, if $f(x)<f\left(z_{1}\right)$, one obtains in the limit $h \rightarrow 0$ :

$$
\mathbb{P}_{x}\left[X_{\tau_{\left(z_{1}, z_{2}\right)}}=z_{2}\right]=-\frac{f^{\prime}\left(z_{2}\right)}{f^{\prime}\left(z_{1}\right)} e^{-\frac{2}{h}\left(f\left(z_{2}\right)-f\left(z_{1}\right)\right)}(1+O(h)),
$$

if $f(x)=f\left(z_{1}\right), x \neq z_{1}$, it holds in the limit $h \rightarrow 0$ :

$$
\mathbb{P}_{x}\left[X_{\tau_{\left(z_{1}, z_{2}\right)}}=z_{2}\right]=f^{\prime}\left(z_{2}\right)\left(\frac{1}{f^{\prime}(x)}-\frac{1}{f^{\prime}\left(z_{1}\right)}\right) e^{-\frac{2}{h}\left(f\left(z_{2}\right)-f\left(z_{1}\right)\right)}(1+O(h)),
$$

and if $f(x)>f\left(z_{1}\right)$, it holds in the limit $h \rightarrow 0$ :

$$
\mathbb{P}_{x}\left[X_{\tau_{\left(z_{1}, z_{2}\right)}}=z_{2}\right]=\frac{f^{\prime}\left(z_{2}\right)}{f^{\prime}(x)} e^{-\frac{2}{h}\left(f\left(z_{2}\right)-f(x)\right)}(1+O(h)) .
$$

Therefore, in dimension one, the estimate (27) holds if and only if $x \in f^{-1}\left(\left(-\infty, f\left(z_{1}\right)\right)\right)$. In accordance with Corollary 16, the asymptotic (27) only holds for initial conditions 
which are sufficiently low in energy. However, we observe that in this simple onedimensional setting, the assumption (26) is not needed. We do not know if the result of Corollary 16 would hold in general without the assumption 26.

Let us now discuss the assumption (21) in the framework of Theorem 1 and Corollary 14. From (13), one has:

$$
\mathbb{P}_{\nu_{h}}\left[X_{\tau_{\left(z_{1}, z_{2}\right)}}=z_{2}\right]=\frac{\int_{z_{1}}^{z_{2}} u_{h} w_{h} e^{-\frac{2}{h} f}}{\int_{z_{1}}^{z_{2}} u_{h} e^{-\frac{2}{h} f}} .
$$

Using Lemma 85, Lemma 26 and (60), one has for some $c>0$, for any $\delta>0$ and for $h$ small enough:

$$
u_{h}(x)=\frac{\chi(x)}{\sqrt{\int_{z_{1}}^{z_{2}} \chi^{2} e^{-\frac{2}{h} f}}}\left(1+\alpha_{h}\right)+r(x), \text { for } x \in \bar{\Omega}
$$

with $\alpha_{h} \in \mathbb{R}, \alpha_{h}=O\left(e^{-\frac{c}{h}}\right), \int_{z_{1}}^{z_{2}} r^{2} e^{-\frac{2}{h} f}=O\left(e^{-\frac{2}{h}\left(f\left(z_{1}\right)-f\left(x_{0}\right)-\delta\right)}\right)$ and where $\chi \in$ $C_{c}^{\infty}\left(z_{1}, z_{2}\right)$ is given by Lemma 85 . Therefore, one has:

$\mathbb{P}_{\nu_{h}}\left[X_{\tau_{\left(z_{1}, z_{2}\right)}}=z_{2}\right]=\frac{1}{\int_{z_{1}}^{z_{2}} u_{h} e^{-\frac{2}{h} f}}\left[\frac{\int_{z_{1}}^{z_{2}} \chi(x) \int_{z_{1}}^{x} e^{\frac{2}{h}(f(y)-f(x))} d y d x}{\int_{z_{1}}^{z_{2}} e^{\frac{2}{h} f} \sqrt{\int_{z_{1}}^{z_{2}} \chi^{2} e^{-\frac{2}{h} f}}}\left(1+\alpha_{h}\right)+\int_{z_{1}}^{z_{2}} r w_{h} e^{-\frac{2}{h} f}\right]$.

Using Proposition 12 and Laplace's method, one gets for any $\delta>0$, in the limit $h \rightarrow 0$ :

$\mathbb{P}_{\nu_{h}}\left[X_{\tau_{\left(z_{1}, z_{2}\right)}}=z_{2}\right]=-\frac{f^{\prime}\left(z_{2}\right)}{f^{\prime}\left(z_{1}\right)} e^{-\frac{2}{h}\left(f\left(z_{2}\right)-f\left(z_{1}\right)\right)}(1+O(h))+O\left(e^{-\frac{1}{h}\left(f\left(z_{2}\right)-f\left(x_{0}\right)+f\left(z_{1}\right)-f\left(x_{0}\right)-\delta\right)}\right)$.

Therefore, the result of Corollary 14 holds if

$$
2\left(f\left(z_{1}\right)-f\left(x_{0}\right)\right)>f\left(z_{2}\right)-f\left(z_{1}\right) .
$$

This explicit computation in dimension one shows that the result of Corollary 1 indeed requires an assumption of the type: the height of the energy barrier to leave the well $f\left(z_{1}\right)-f\left(x_{0}\right)$ is sufficiently large compared to the largest difference in energy of the saddle points $f\left(z_{2}\right)-f\left(z_{1}\right)$. Notice that (28) differs from (21) by a multiplicative factor $\frac{1}{2}$. We do not know if the result of Corollary 14 would hold in general under the weaker assumption (28). Finally, let us mention that when $d=1,(20)$ is always satisfied.

\subsubsection{Extension of the results to a subset of generalized saddle points and to more general subsets of $\partial \Omega$}

It is actually possible to generalize the result of Theorem 1 and Corollary 14 to less stringent conditions than (20)-(21) and to more general subsets $\Sigma \subset \partial \Omega$.

Theorem 2. Assume that [H1], [H2] and [H3] hold. Assume that there exist $k_{0} \in$ $\{1, \ldots, n\}$ and $f^{*} \in \mathbb{R}$ such that $f\left(z_{k_{0}}\right) \leq f^{*} \leq f\left(z_{k_{0}+1}\right)$ (with the convention $f\left(z_{k_{0}+1}\right)=$ $+\infty$ if $k_{0}=n$ ),

$$
\left\{\begin{array}{l}
\forall i \in\left\{1, \ldots, k_{0}\right\}, \inf _{z \in B_{z_{i}}^{c}} d_{a}\left(z, z_{i}\right)>\max \left[f^{*}-f\left(z_{i}\right), f\left(z_{i}\right)-f\left(z_{1}\right)\right] \\
\forall i \in\left\{k_{0}+1, \ldots, n\right\}, \inf _{z \in B_{z_{i}}^{c}} d_{a}\left(z, z_{i}\right)>f^{*}-f\left(z_{1}\right)
\end{array}\right.
$$


and,

$$
f\left(z_{1}\right)-f\left(x_{0}\right)>f^{*}-f\left(z_{1}\right) .
$$

Let $u_{h}$ be the eigenfunction associated with the smallest eigenvalue of $-L_{f, h}^{D,(0)}(\Omega)$ (see Proposition 4) which satisfies (11).

1. For all $i \in\left\{1, \ldots, k_{0}\right\}$ and for all smooth open set $\Sigma_{i} \subset \partial \Omega$ containing $z_{i}$ and such that $\bar{\Sigma}_{i} \subset B_{z_{i}}$, the limit (22) holds for $\int_{\Sigma_{i}}\left(\partial_{n} u_{h}\right) e^{-\frac{2}{h} f} d \sigma$ and the limit (24) holds for $\mathbb{P}_{\nu_{h}}\left[X_{\tau_{\Omega}} \in \Sigma_{i}\right]$. Moreover, if $f\left(z_{k_{0}+1}\right)>f\left(z_{k_{0}}\right)$, for all $i \in\left\{k_{0}+1, \ldots, n\right\}$ and for all smooth open set $\Sigma_{i} \subset \partial \Omega$ containing $z_{i}$ and such that $\bar{\Sigma}_{i} \subset B_{z_{i}}$, there exist $\varepsilon>0$ and $h_{0}>0$ such that for all $h \in\left(0, h_{0}\right)$

$$
\int_{\Sigma_{i}}\left(\partial_{n} u_{h}\right) e^{-\frac{2}{h} f} d \sigma=\left(\int_{\Sigma_{k_{0}}}\left(\partial_{n} u_{h}\right) e^{-\frac{2}{h} f} d \sigma\right) O\left(e^{-\frac{\varepsilon}{h}}\right)
$$

and

$$
\mathbb{P}_{\nu_{h}}\left[X_{\tau_{\Omega}} \in \Sigma_{i}\right]=\mathbb{P}_{\nu_{h}}\left[X_{\tau_{\Omega}} \in \Sigma_{k_{0}}\right] O\left(e^{-\frac{\varepsilon}{h}}\right)
$$

2. Let $j_{0} \in\left\{1, \ldots, k_{0}\right\}$ and $\Sigma \subset \partial \Omega$ be a smooth open set such that $\bar{\Sigma} \subset B_{z_{j_{0}}}$ and $\inf _{\Sigma} f=f^{*}$. Let $\left(B^{*}, p^{*}\right) \in \mathbb{R}_{+}^{*} \times \mathbb{R}$ be such that

$$
\int_{\Sigma}\left(\partial_{n} f\right) e^{-\frac{2}{h} f} d \sigma=B^{*} h^{p^{*}} e^{-\frac{2}{h} f^{*}}(1+O(h)) .
$$

Then, one obtains in the limit $h \rightarrow 0$

$$
\int_{\Sigma}\left(\partial_{n} u_{h}\right) e^{-\frac{2}{h} f} d \sigma=-\frac{2 B^{*}\left(\operatorname{det} \operatorname{Hess} f\left(x_{0}\right)\right)^{\frac{1}{4}}}{\pi^{\frac{d}{4}}} h^{p^{*}-\frac{d}{4}-1} e^{-\frac{1}{h}\left(2 f^{*}-f\left(x_{0}\right)\right)}(1+O(h))
$$

and

$$
\mathbb{P}_{\nu_{h}}\left[X_{\tau_{\Omega}} \in \Sigma\right]=\frac{B^{*}}{\pi^{\frac{d-1}{2}}}\left(\sum_{k=1}^{n_{0}} \frac{\partial_{n} f\left(z_{k}\right)}{\sqrt{\operatorname{det} \operatorname{Hess} f_{\mid \partial \Omega}\left(z_{k}\right)}}\right)^{-1} h^{p^{*}-\frac{d-1}{2}} e^{-\frac{2}{h}\left(f^{*}-f\left(z_{1}\right)\right)}(1+O(h)) .
$$

In practice, the expansion (33) is given by Laplace's method. Theorem 2 is a generalization of Theorem 1. Indeed, (29)-(30) is weaker than (20)- 21) (20)- 21 implies 299)-(30) for $k_{0}=n$ and $\left.f^{*}=f\left(z_{n}\right)\right)$ and item 2 gives an asymptotic result on the exit probability through $\Sigma \subset B_{z_{j_{0}}}$ even if $z_{j_{0}} \notin \bar{\Sigma}$.

As an illustration, let us state a corollary of this theorem, which demonstrates the importance of obtaining a precise asymptotic result including the prefactors. Let us consider a simple situation with only two local minima $z_{1}$ and $z_{2}$ on the boundary, with $f\left(z_{1}\right)<f\left(z_{2}\right)$. Let us now compare the two exit probabilities (see Figure 7 for a schematic representation of the geometric setting):

- The probability to leave through $\Sigma_{2}$ such that $\overline{\Sigma_{2}} \subset B_{z_{2}}$ and $z_{2} \in \Sigma_{2}$;

- The probability to leave through $\Sigma$ such that $\bar{\Sigma} \subset B_{z_{1}}$ and $\inf _{\Sigma} f=f\left(z_{2}\right)$.

By classic results from the large deviation theory (see for example (8)) the probability to exit through $\Sigma$ and $\Sigma_{2}$ both scale like a prefactor times $\mathrm{e}^{-\frac{2}{h}\left(f\left(z_{2}\right)-f\left(z_{1}\right)\right)}$ : the difference 
can only be read from the prefactors. Actually, using item 2 in Theorem 2 , one obtains the existence of $C>0$ such that in the limit $h \rightarrow 0$ (see Corollary 17 below),

$$
\frac{\mathbb{P}_{\nu_{h}}\left(X_{\tau_{\Omega}} \in \Sigma\right)}{\mathbb{P}_{\nu_{h}}\left(X_{\tau_{\Omega}} \in \Sigma_{2}\right)} \sim C \sqrt{h}
$$

The probability to leave through $\Sigma_{2}$ (namely through the generalized saddle point $z_{2}$ ) is thus larger than through $\Sigma$, even though the two regions are at the same height. This result explains why the local minima of $f$ on the boundary (namely the generalized saddle points) play such an important role when studying the exit event. Let us now state the precise result.

Corollary 17. Assume [H1], [H2], [H3]. Assume that $\left.f\right|_{\partial \Omega}$ has only two local minima $z_{1}$ and $z_{2}$ such that $f\left(z_{1}\right)<f\left(z_{2}\right)$ and,

$$
\text { for } j \in\{1,2\}, \inf _{z \in B_{z_{j}}^{c}} d_{a}\left(z, z_{j}\right)>f\left(z_{2}\right)-f\left(z_{1}\right),
$$

and

$$
f\left(z_{1}\right)-f\left(x_{0}\right)>f\left(z_{2}\right)-f\left(z_{1}\right) .
$$

Let $\Sigma \subset \partial \Omega$ be a smooth open set such that $\bar{\Sigma} \subset B_{z_{1}}$. Assume moreover that $\inf _{\Sigma} f=$ $f\left(z_{2}\right)$ and that the infimum is attained at a single point $z^{*}: \inf _{\Sigma} f=f\left(z^{*}\right)$ (necessarily $\left.z^{*} \in \partial \Sigma\right)$. Finally, let us assume that $z^{*}$ is a non degenerate minimum of $f_{\mid \partial \Sigma}$ and $\partial_{n(\partial \Sigma)} f_{\mid \partial \Sigma}\left(z^{*}\right)<0$ where $n(\partial \Sigma)$ is the unit outward normal to $\partial \Sigma \subset \partial \Omega$. Then, one has the following asymptotic expansion of $\mathbb{P}_{\nu_{h}}\left[X_{\tau_{\Omega}} \in \Sigma\right]$ in the limit $h \rightarrow 0$ :

$$
\begin{aligned}
\mathbb{P}_{\nu_{h}}\left[X_{\tau_{\Omega}} \in \Sigma\right]= & -\frac{\sqrt{h}}{2 \sqrt{\pi}} \frac{\partial_{n} f\left(z^{*}\right)}{\partial_{n(\partial \Sigma)} f\left(z^{*}\right) \sqrt{\operatorname{det} \operatorname{Hess} f_{\mid \partial \Sigma}\left(z^{*}\right)}}\left(\sum_{k=1}^{n_{0}} \frac{\partial_{n} f\left(z_{k}\right)}{\sqrt{\operatorname{det} \operatorname{Hess} f_{\mid \partial \Omega}\left(z_{k}\right)}}\right)^{-1} \\
& \times e^{-\frac{2}{h}\left(f\left(z_{2}\right)-f\left(z_{1}\right)\right)}(1+O(h)),
\end{aligned}
$$

with by convention, $\operatorname{det} \operatorname{Hess} f_{\mid \partial \Sigma}\left(z^{*}\right)=1$ if $d=2$.

Corollaries 14, 16 and 17 imply the result (36) announced above.

Remark 18. By using Laplace's method, one can check that the asymptotic results obtained in Corollaries 14, 16 and 17 on the law of $X_{\tau_{\Omega}}$ imply that the density of $X_{\tau_{\Omega}}$ with respect to the Lebesgue measure on $\partial \Omega$ is, in the limit $h \rightarrow 0$,

$$
z \in \partial \Omega \mapsto \frac{\partial_{n} f(z) e^{-\frac{2}{h} f(z)}}{\int_{\partial \Omega} \partial_{n} f e^{-\frac{2}{h} f} d \sigma}(1+O(h)) .
$$

This indeed yields the same asymptotic limits on the exit distribution. This is reminiscent of previous results obtained in [19, 47, 68], where the authors proved that, starting from a deterministic initial condition in $\Omega, X_{\tau_{\Omega}}$ has a density with respect to the Lebesgue measure on $\partial \Omega$ which satisfies, in the limit $h \rightarrow 0, z \in \partial \Omega \mapsto \frac{\partial_{n} f(z) e^{-\frac{2}{h} f(z)}}{\int_{\partial \Omega} \partial_{n} f e^{-\frac{2}{h} f} d \sigma}+o(1)$, which is however a less precise estimate than (39). 


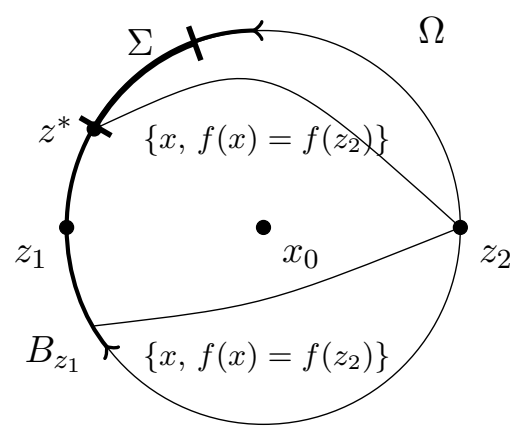

Figure 7: Schematic representation of the geometric setting of Corollary 17. The subset $\Sigma$ is such that $\bar{\Sigma} \subset B_{z_{1}}$ and $\inf _{\Sigma} f=f\left(z_{2}\right)$.

\subsection{Strategy for the proof of Theorem 1 and outline of the paper}

The aim of this section is to give an overview of the strategy for the proof of Theorem 1 . In view of $(22)$, we would like to identify the asymptotic behavior of the normal derivative $\partial_{n} u_{h}$ on $\partial \Omega$ in the limit $h \rightarrow 0$. We recall that $\left(\lambda_{h}, u_{h}\right)$ satisfies the eigenvalue problem (12). By differentiating (12), we observe that $\nabla u_{h}$ satisfies

$$
\left\{\begin{aligned}
L_{f, h}^{(1)} \nabla u_{h} & =-\lambda_{h} \nabla u_{h} \text { on } \Omega, \\
\nabla_{T} u_{h} & =0 \text { on } \partial \Omega, \\
\left(\frac{h}{2} \operatorname{div}-\nabla f \cdot\right) \nabla u_{h} & =0 \text { on } \partial \Omega,
\end{aligned}\right.
$$

where

$$
L_{f, h}^{(1)}=\frac{h}{2} \Delta-\nabla f \cdot \nabla-\operatorname{Hess} f
$$

is an operator acting on 1-forms (namely on vector fields). Therefore $\nabla u_{h}$ is an eigen1-form of the operator $-L_{f, h}^{(1)}$ with tangential Dirichlet boundary conditions (see 40p), associated with the small eigenvalue $\lambda_{h}$.

It is known (see for example 39 ) that in our geometric setting, $-L_{f, h}^{D,(0)}(\Omega)$ admits exactly one eigenvalue smaller than $\frac{\sqrt{h}}{2}$, namely $\lambda_{h}$ with associated eigenfunction $u_{h}$ (this is because $f$ has only one local minimum in $\Omega$ ) and that $-L_{f, h}^{D,(1)}(\Omega)$ admits exactly $n$ eigenvalues smaller than $\frac{\sqrt{h}}{2}$ (where, we recall, $n$ is the number of local minima of $f$ on $\partial \Omega$ ). Actually, all these small eigenvalues are exponentially small in the regime $h \rightarrow 0$, the other eigenvalues being bounded from below by a constant in this regime. The idea is then to construct an appropriate basis (with so called quasi-modes, which are localized on the generalized saddle points $\left.\left(z_{i}\right)_{i=1, \ldots, n}\right)$ of the eigenspace associated with small eigenvalues for $L_{f, h}^{D,(1)}(\Omega)$, and then to decompose $\nabla u_{h}$ along this basis.

The article is organized as follows. In Section 2, we introduce the general setting for the proof of our results, and the Gram-Schmidt procedure which allows, starting from a set of quasi-modes, to compute the projection of (an approximation of) $\nabla u_{h}$ along the quasi-modes. In order to quantify the distance between the space spanned by these quasi-modes and the eigenspace of $L_{f, h}^{D,(1)}(\Omega)$ associated with small eigenvalues, we need to use so-called Agmon estimates. Section 3 is devoted to a presentation of the main properties of the Agmon distance which intervenes in these estimates. The 
most technical part is the effective construction of the quasi-modes using auxiliary simpler eigenvalue problems associated with each of the local minima $\left(z_{i}\right)_{i=1, \ldots, n}$. This is explained in Section 4 which concludes the proof of Theorem 1. Finally, Section 5 concludes the paper by providing the proofs of all the other results stated above, in particular Theorem 2 .

For the ease of the reader, a list of the main notation used in this work is provided at the end of this work.

\section{General setting and strategy for the proof of Theorem 1}

The general setting for proving the results presented in Section 1 will be the following: $\bar{\Omega}$ is a $C^{\infty}$ oriented connected compact Riemannian manifold of dimension $d$ with boundary $\partial \Omega$ and the function $f$ is a $C^{\infty}$ real valued function defined on $\bar{\Omega}$. One defines $\Omega:=$ $\bar{\Omega} \backslash \partial \Omega$. In particular, Theorem 1 will actually be proven in this framework. Notice that the assumptions [H1], [H2] and [H3] are still meaningful in this more general setting.

In order to use previous results from the literature on semi-classical analysis, we will transform the original problem $(12)$ on $\left(\lambda_{h}, u_{h}\right)$ associated with weighted Hilbert space $H_{w}^{q}(\Omega)$ to an eigenvalue problem on the standard (non-weighted) Hilbert spaces $H^{q}(\Omega)$, by using a unitary transformation which relates the operator $L_{f, h}^{(p)}$ to the Witten Laplacians $\Delta_{f, h}^{(p)}$. This is explained in Section 2.1, together with some first well-known results on the spectrum of Witten Laplacians. Then, in Section 2.2, we explain what are the requirements on the quasi-modes we will build in order to obtain the estimate (22), see Proposition 25. Section 2.3 is finally devoted to the proof of Proposition 25.

\subsection{Witten Laplacians}

\subsubsection{Notations for Sobolev spaces}

For $p \in\{0, \ldots, d\}$, one denotes by $\Lambda^{p} C^{\infty}(\bar{\Omega})$ the space of $C^{\infty} p$-forms on $\bar{\Omega}$. Moreover, $\Lambda^{p} C_{T}^{\infty}(\bar{\Omega})$ is the set of $C^{\infty} p$-forms $v$ such that $\mathbf{t} v=0$ on $\partial \Omega$, where $\mathbf{t}$ denotes the tangential trace on forms (see for instance $[74$ p. 27$]$ ). Likewise, the set $\Lambda^{p} C_{N}^{\infty}(\bar{\Omega})$ is the set of $C^{\infty} p$-forms $v$ such that $\mathbf{n} v=0$ on $\partial \Omega$, where $\mathbf{n}$ denotes the normal trace on forms defined by: for all $w \in \Lambda^{p} C^{\infty}(\bar{\Omega}), \mathbf{n} w:=\left.w\right|_{\partial \Omega}-\mathbf{t} w$.

For $p \in\{0, \ldots, d\}$ and $q \in \mathbb{N}$, one denotes by $\Lambda^{p} H_{w}^{q}(\Omega)$ the weighted Sobolev spaces of $p$ forms with regularity index $q$, for the weight $e^{-\frac{2}{h} f(x)} d x$ on $\Omega: v \in \Lambda^{p} H_{w}^{q}(\Omega)$ if and only if for all multi-index $\alpha$ with $|\alpha| \leq q$, the $\alpha$ derivative of $v$ is in $\Lambda^{p} L_{w}^{2}(\Omega)$ where $\Lambda^{p} L_{w}^{2}(\Omega)$ is the completion of the space $\Lambda^{p} C^{\infty}(\bar{\Omega})$ for the norm

$$
w \in \Lambda^{p} C^{\infty}(\bar{\Omega}) \mapsto \sqrt{\int_{\Omega}|w|^{2} e^{-\frac{2}{h} f}} .
$$

See for example $[74$ for an introduction to Sobolev spaces on manifolds with boundaries. For $p \in\{0, \ldots, d\}$ and $q>\frac{1}{2}$, the set $\Lambda^{p} H_{w, T}^{q}(\Omega)$ is defined by

$$
\Lambda^{p} H_{w, T}^{q}(\Omega):=\left\{v \in \Lambda^{p} H_{w}^{q}(\Omega) \mid \mathbf{t} v=0 \text { on } \partial \Omega\right\} .
$$

Notice that $\Lambda^{p} L_{w}^{2}(\Omega)$ is the space $\Lambda^{p} H_{w}^{0}(\Omega)$, and that $\Lambda^{0} H_{w, T}^{1}(\Omega)$ is the space $H_{w, 0}^{1}(\Omega)$ that we introduced in Proposition 3 to define the domain of $L_{f, h}^{D,(0)}(\Omega)$. Likewise for 
$p \in\{0, \ldots, d\}$ and $q>\frac{1}{2}$, the set $\Lambda^{p} H_{w, N}^{q}(\Omega)$ is defined by

$$
\Lambda^{p} H_{w, N}^{q}(\Omega):=\left\{v \in \Lambda^{p} H_{w}^{q}(\Omega) \mid \mathbf{n} v=0 \text { on } \partial \Omega\right\} .
$$

We will denote by $\|\cdot\|_{H_{w}^{q}}$ the norm on the weighted space $\Lambda^{p} H_{w}^{q}(\Omega)$. Moreover $\langle\cdot, \cdot\rangle_{L_{w}^{2}}$ denotes the scalar product in $\Lambda^{p} L_{w}^{2}(\Omega)$.

Finally, we will also use the same notation without the index $w$ to denote the standard Sobolev spaces defined with respect to the Lebesgue measure on $\Omega$.

\subsubsection{Witten Laplacians on a manifold with boundary}

Let us first recall some basic properties of Witten Laplacians, as well as the link between those and the operators $L_{f, h}^{(p)}$ introduced above (see 10 and 41$)$ ). As already explained above, we will actually need in this article to work only with 0 and 1-forms $(p \in\{0,1\})$. For an introduction to the Hodge theory and the Hodge Laplacians on manifolds with boundary, one can refer to 74 .

Denote by $d$ the exterior derivative on $\Omega$,

$$
d^{(p)}: \Lambda^{p} C^{\infty}(\Omega) \rightarrow \Lambda^{p+1} C^{\infty}(\Omega),
$$

and $\left(d^{(p)}\right)^{*}$ its adjoint. The exterior derivative is 2 nilpotent,

$$
d^{(p+1)} \circ d^{(p)}=0,
$$

and therefore $\left(d^{(p)}\right)^{*} \circ\left(d^{(p+1)}\right)^{*}=0$. In all what follows, the superscript $(p)$ may be omitted when there is no ambiguity.

Let us now introduce the so called distorted exterior derivative

$$
d_{f, h}^{(p)}:=e^{-\frac{f}{h}} h d^{(p)} e^{\frac{f}{h}}=h d^{(p)}+d f \wedge
$$

and its formal adjoint

$$
\left(d_{f, h}^{(p)}\right)^{*}:=e^{\frac{f}{h}} h\left(d^{(p)}\right)^{*} e^{\frac{-f}{h}}=h\left(d^{(p)}\right)^{*}+\mathbf{i}_{\nabla f} .
$$

The distorted exterior derivative was firstly introduced by Witten in [83].

Definition 19. The Witten Laplacian is the non negative differential operator

$$
\Delta_{f, h}^{(p)}:=\left(d_{f, h}^{(p)}+\left(d_{f, h}^{(p)}\right)^{*}\right)^{2} .
$$

An equivalent expression of the Witten Laplacians is

$$
\Delta_{f, h}^{(p)}=h^{2} \Delta_{H}^{(p)}+|\nabla f|^{2}+h\left(\mathcal{L}_{\nabla f}+\mathcal{L}_{\nabla f}^{*}\right),
$$

where $\mathcal{L}$ stands for the Lie derivative, $\nabla$ is the covariant derivative associated to the metric on $\Omega$ and $\Delta_{H}^{(p)}$ is the Hodge Laplacian acting on $p$-forms, defined by:

$$
\Delta_{H}^{(p)}:=\left(d^{(p)}+\left(d^{(p)}\right)^{*}\right)^{2}
$$

We recall that $\Delta_{H}^{(p)}$ is a positive operator (in $\mathbb{R}^{d}, \Delta_{H}^{(0)}=-\sum_{i=1}^{d} \partial_{x_{i}, x_{i}}^{2}$ ). The operator $\mathcal{L}_{\nabla f}+\mathcal{L}_{\nabla f}^{*}$ is an operator of order 0 (namely a multiplicative operator). On 0 -forms, namely on functions, the Witten Laplacian has the following expression

$$
\Delta_{f, h}^{(0)}=h^{2} \Delta_{H}^{(0)}+|\nabla f|^{2}+h \Delta_{H}^{(0)} f .
$$

Let us now make precise the natural Dirichlet and Neumann boundary conditions for Witten Laplacians on a manifold with boundary (see [39]). 
Proposition 20. The Dirichlet realization of $\Delta_{f, h}^{(p)}$ on $\Omega$ is the operator $\Delta_{f, h}^{D,(p)}(\Omega)$ with domain

$$
D\left(\Delta_{f, h}^{D,(p)}(\Omega)\right)=\left\{v \in \Lambda^{p} H^{2}(\Omega) \mid \mathbf{t} v=0, \mathbf{t} d_{f, h}^{*} v=0\right\} .
$$

The Neumann realization of $\Delta_{f, h}^{(p)}$ on $\Omega$ is the operator $\Delta_{f, h}^{N,(p)}(\Omega)$ with domain

$$
D\left(\Delta_{f, h}^{N,(p)}(\Omega)\right)=\left\{v \in \Lambda^{p} H^{2}(\Omega) \mid \mathbf{n} v=0, \mathbf{n} d_{f, h} v=0\right\} .
$$

The operators $\Delta_{f, h}^{D,(p)}(\Omega)$ and $\Delta_{f, h}^{N,(p)}(\Omega)$ are both self adjoint operators with compact resolvent.

We recall that $\mathbf{t}$ denotes the tangential trace on forms and that $\mathbf{n} \omega=\omega-\mathbf{t} \omega$ is the normal trace. The proof of Proposition 20 can be found in [39, Section 2.4] and in [54, Section 4.2.3]. It is a generalization of what is stated in 74 for the Hodge Laplacians. One can check that the operator $\Delta_{f, h}^{D,(p)}(\Omega)$ is actually the Friedrichs extension associated to the quadratic form

$$
v \in \Lambda^{p} H_{T}^{1}(\Omega) \mapsto\left\|d_{f, h}^{(p)} v\right\|_{L^{2}}^{2}+\left\|\left(d_{f, h}^{(p)}\right)^{*} v\right\|_{L^{2}}^{2} .
$$

The following properties are easily checked for $v \in D\left(\Delta_{f, h}^{D,(p)}(\Omega)\right)$ such that $d_{f, h} v \in$ $D\left(\Delta_{f, h}^{D,(p+1)}(\Omega)\right)$ and $d_{f, h}^{*} v \in D\left(\Delta_{f, h}^{D,(p-1)}(\Omega)\right)$ :

$$
d_{f, h} \Delta_{f, h}^{D,(p)}(\Omega) v=\Delta_{f, h}^{D,(p+1)}(\Omega) d_{f, h} v \text { and } d_{f, h}^{*} \Delta_{f, h}^{D,(p)}(\Omega) v=\Delta_{f, h}^{D,(p-1)}(\Omega) d_{f, h}^{*} v .
$$

Similar relations hold for $\Delta_{f, h}^{N}(\Omega)$.

One can relate the infinitesimal generator $L_{f, h}^{(0)}$ of the diffusion 11 to the Witten Laplacian $\Delta_{f, h}^{(0)}$ through the unitary transformation:

$$
\phi \in L_{w}^{2}(\Omega) \mapsto e^{-\frac{f}{h}} \phi \in L^{2}(\Omega) .
$$

Indeed, one can check that

$$
\Delta_{f, h}^{D,(0)}(\Omega)=-2 h e^{-\frac{f}{h}} L_{f, h}^{D,(0)}(\Omega) e^{\frac{f}{h}} .
$$

Let us now generalize this to $p$-forms, using extensions of $L_{f, h}^{(0)}$ to $p$-forms.

Proposition 21. The Friedrichs extension associated with the quadratic form

$$
v \in \Lambda^{p} C_{T}^{\infty}(\Omega) \mapsto \frac{h}{2}\left[\left\|d^{(p)} v\right\|_{L_{w}^{2}(\Omega)}^{2}+\left\|e^{\frac{2 f}{h}}\left(d^{(p)}\right)^{*} e^{-\frac{2 f}{h}} v\right\|_{L_{w}^{2}(\Omega)}^{2}\right]
$$

on $\Lambda^{p} L_{w}^{2}(\Omega)$, is denoted $\left(-L_{f, h}^{D,(p)}(\Omega), D\left(-L_{f, h}^{D,(p)}(\Omega)\right)\right)$. The operator $-L_{f, h}^{D,(p)}(\Omega)$ is a positive unbounded self adjoint operator on $\Lambda^{p} L_{w}^{2}(\Omega)$. Besides, one has

$$
D\left(-L_{f, h}^{D,(p)}(\Omega)\right)=\left\{v \in \Lambda^{p} H_{w}^{2}(\Omega) \mid \mathbf{t} v=0, \mathbf{t} d^{*}\left(e^{-\frac{2 f}{h}} v\right)=0\right\} .
$$

For $p=0$, the differential operator

$$
L_{f, h}^{(0)}=-\frac{h}{2} \Delta_{H}^{(0)}-\nabla f \cdot \nabla
$$


is the infinitesimal generator (10) of the overdamped Langevin dynamics (1). For $p=1$ one gets the operator already introduced in (41):

$$
L_{f, h}^{(1)}=-\frac{h}{2} \Delta_{H}^{(1)}-\nabla f \cdot \nabla-\operatorname{Hess} f,
$$

where we recall Hess $f$ is the Hessian of $f$, see Remark 10. The generalisation of (47) to $p$-forms is:

$$
\Delta_{f, h}^{D,(p)}(\Omega)=-2 h e^{-\frac{f}{h}}\left(L_{f, h}^{D,(p)}(\Omega)\right) e^{\frac{f}{h}} .
$$

The intertwining relation 46 writes on $L_{f, h}^{D,(p)}(\Omega)$ :

$$
L_{f, h}^{D,(p+1)}(\Omega) d=d L_{f, h}^{D,(p)}(\Omega) \text { and } L_{f, h}^{D,(p-1)}(\Omega) d_{2 f, h}^{*}=d_{2 f, h}^{*} L_{f, h}^{D,(p)}(\Omega) .
$$

Thanks to the relation (49), the operators $L_{f, h}^{D,(p)}(\Omega)$ and $\Delta_{f, h}^{D,(p)}(\Omega)$ have the same spectral properties. In particular the operators $L_{f, h}^{D,(p)}(\Omega)$ and $\Delta_{f, h}^{D,(p)}(\Omega)$ both have compact resolvents, and thus a discrete spectrum. The generalization of Proposition 4 is the following:

Proposition 22. The smallest eigenvalue of $-L_{f, h}^{D,(0)}(\Omega)$, denoted by $\lambda_{h}$, is positive and non degenerate. The associated eigenfunction $u_{h}$ has sign on $\Omega$. Moreover $u_{h} \in$ $C^{\infty}(\bar{\Omega}, \mathbb{R})$.

Without loss of generality, one can assume that $u_{h}$ satisfies (11). Thanks to the relation (49), the couple $\left(\mu_{h}, v_{h}\right):=\left(2 h \lambda_{h}, u_{h} e^{-\frac{f}{h}}\right)$ is the first eigenvalue and eigenfunction of $\Delta_{f, h}^{D,(0)}(\Omega)$. The couple $\left(\mu_{h}, v_{h}\right)$ satisfies

$$
\left\{\begin{aligned}
\Delta_{f, h}^{(0)} v_{h} & =\mu_{h} v_{h} \text { on } \Omega, \\
v_{h} & =0 \text { on } \partial \Omega .
\end{aligned}\right.
$$

Moreover, $v_{h}>0$ on $\Omega$ and

$$
\int_{\Omega} v_{h}^{2}(x) d x=1
$$

The following lemma, which is a direct consequence of the spectral theorem (see for instance [37, Theorem 8.15]), will be instrumental throughout this work.

Lemma 23. Let $(A, D(A))$ be a non negative self adjoint operator on a Hilbert Space $(\mathcal{H},\|\|$.$) with associated quadratic form q_{A}(u)=(u, A u)$ with domain $D\left(q_{A}\right)$. Then for any $u \in D\left(q_{A}\right)$ and $b>0$

$$
\left\|\pi_{[b,+\infty)}(A) u\right\|^{2} \leq \frac{q_{A}(u)}{b}
$$

where, for $E \subset \mathbb{R}$ a Borel set, $\pi_{E}(A)$ is the spectral projection of the operator $A$ on $E$.

This lemma will be in particular applied to the non negative self adjoint operators $\Delta_{f, h}^{D,(p)}(\Omega)$ and $-L_{f, h}^{D,(p)}(\Omega)$ and their associated quadratic forms. 


\subsubsection{Small eigenvalues of $\Delta_{f, h}^{D,(0)}(\Omega)$ and $L_{f, h}^{D,(0)}(\Omega)$}

According to [39, Corollary 2.4.4], the following relations hold for all $v \in \Lambda^{p} H_{T}^{1}(\Omega)$ :

$$
\pi_{\left[0, h^{3 / 2}\right)}\left(\Delta_{f, h}^{D,(p+1)}(\Omega)\right) d_{f, h} v=d_{f, h} \pi_{\left[0, h^{3 / 2}\right)}\left(\Delta_{f, h}^{D,(p)}(\Omega)\right) v,
$$

and

$$
\pi_{\left[0, h^{3 / 2}\right)}\left(\Delta_{f, h}^{D,(p-1)}(\Omega)\right) d_{f, h}^{*} v=d_{f, h}^{*} \pi_{\left[0, h^{3 / 2}\right)}\left(\Delta_{f, h}^{D,(p)}(\Omega)\right) v .
$$

For $p \in\{0, \ldots, n\}$, let us define $F_{h}^{(p)}:=\operatorname{Ran}\left(\pi_{\left[0, h^{3 / 2}\right)}\left(\Delta_{f, h}^{D,(p)}(\Omega)\right)\right)$. Then, according to the previous intertwining relations, one can define a finite dimensional Dirichlet complex structure (see [12], [39] and [52]):

$$
\begin{gathered}
\{0\} \longrightarrow F_{h}^{(0)} \stackrel{d_{f, h}}{\longrightarrow} F_{h}^{(1)} \stackrel{d_{f, h}}{\longrightarrow} \cdots F_{h}^{(d)} \stackrel{d_{f, h}}{\longrightarrow}\{0\} \\
\{0\} \stackrel{d_{f, h}^{*}}{\longleftarrow} F_{h}^{(0)} \stackrel{d_{f, h}^{*}}{\longleftarrow} F_{h}^{(1)} \cdots \stackrel{d_{f, h}^{*}}{\longleftarrow} F_{h}^{(d)} \longleftarrow\{0\} .
\end{gathered}
$$

For $p \in\{0, \ldots, n\}$, the dimension of the vector space $F_{h}^{(p)}$ in the regime $h \rightarrow 0$ have been studied in 39, Section 3] when $\nabla f \neq 0$ on $\partial \Omega$ and when $f: \bar{\Omega} \rightarrow \mathbb{R}$ and $f_{\mid \partial \Omega}$ are Morse functions. In particular, it is proved there that the dimension of $F_{h}^{(0)}$ (respectively $F_{h}^{(1)}$ ) is equal to the number of local minima of $f$ (respectively to the number of generalized critical points of index 1). A generalized critical point of index 1 for $\Delta_{f, h}^{D,(1)}(\Omega)$ is either a local minimum of $\left.f\right|_{\partial \Omega}$ such that $\partial_{n} f\left(z_{i}\right)>0$ or a saddle point of index 1 of $f$ inside $\Omega$. In our setting, thanks to assumptions [H1], [H2] and [H3], there are $n$ generalized critical points of index 1 , which are the local minima $\left(z_{i}\right)_{i=1, \ldots, n}$ of $\left.f\right|_{\partial \Omega}$.

Proposition 24. Under [H1], [H2], and [H3], there exists $h_{0}>0$ such that for all $h \in\left(0, h_{0}\right)$,

$$
\operatorname{dim} F_{h}^{(0)}=1 \quad \text { and } \quad \operatorname{dim} F_{h}^{(1)}=n .
$$

We refer to [39, Theorem 3.2.3] for the proof of this proposition.

From 39, each eigenvalue $\mu$ of $\Delta_{f, h}^{D,(1)}(\Omega)$ which is smaller than $h^{3 / 2}$ is exponentially small when $h \rightarrow 0$, i.e.

$$
\limsup _{h \rightarrow 0} h \ln \mu<0 \text {. }
$$

Thanks to 49 , similar results hold for $L_{f, h}^{D,(p)}(\Omega)$ : there exists $h_{0}>0$ such that for all $h \in\left(0, h_{0}\right)$

$$
\operatorname{dim} \operatorname{Ran} \pi_{\left[0, \frac{\sqrt{h}}{2}\right)}\left(-L_{f, h}^{D,(0)}(\Omega)\right)=1 \text { and } \operatorname{dim} \operatorname{Ran} \pi_{\left[0, \frac{\sqrt{h}}{2}\right)}\left(-L_{f, h}^{D,(1)}(\Omega)\right)=n .
$$

The spectral projection $\pi_{\left[0, \frac{\sqrt{h}}{2}\right)}\left(-L_{f, h}^{D,(0)}(\Omega)\right)$ is the orthogonal projection in $L_{w}^{2}(\Omega)$ on $\operatorname{span}\left(u_{h}\right)$ and thanks to the intertwining property (50), we have the following crucial property:

$$
\nabla u_{h} \in \operatorname{Ran} \pi_{\left[0, \frac{\sqrt{h}}{2}\right)}\left(-L_{f, h}^{D,(1)}(\Omega)\right) .
$$

For the ease of notation, for $p \in\{0,1\}$, we use in the following the notation:

$$
\pi_{h}^{(p)}:=\pi_{\left[0, \frac{\sqrt{h}}{2}\right)}\left(-L_{f, h}^{D,(p)}(\Omega)\right) .
$$




\subsection{Statement of the assumptions required for the quasi-modes}

2.2.1 Assumptions on quasi-modes for $L_{f, h}^{D,(p)}, p \in\{0,1\}$

The next proposition gives the assumption we need on the quasi-modes $\left(\tilde{\psi}_{i}\right)_{i=1, \ldots, n}$ whose span approximates $\operatorname{Ran} \pi_{h}^{(1)}$, and $\tilde{u}$ whose span approximates $\operatorname{Ran} \pi_{h}^{(0)}$, in order to prove Theorem 1 .

Proposition 25. Assume [H1], [H2] and [H3]. As in the statement of Theorem 1 , for all $i \in\{1, \ldots, n\}, \Sigma_{i}$ denotes an open set included in $\partial \Omega$ containing $z_{i}$ and such that $\bar{\Sigma}_{i} \subset B_{z_{i}}$.

Let us assume in addition that there exist $n$ quasi-modes $\left(\tilde{\psi}_{i}\right)_{i=1, \ldots, n}$ and a family of quasi-modes $\left(\tilde{u}=\tilde{u}_{\delta}\right)_{\delta>0}$ satisfying the following conditions:

1. For all $i \in\{1, \ldots, n\}, \tilde{\psi}_{i} \in \Lambda^{1} H_{w, T}^{1}(\Omega)$ and $\tilde{u} \in \Lambda^{0} H_{w, T}^{1}(\Omega)$. The function $\tilde{u}$ is non negative in $\Omega$. Moreover, one assumes the following normalization: for all $i \in\{1, \ldots, n\}$,

$$
\left\|\tilde{\psi}_{i}\right\|_{L_{w}^{2}}=\|\tilde{u}\|_{L_{w}^{2}}=1 \text {. }
$$

2. (a) There exists $\varepsilon_{1}>0$ such that for all $i \in\{1, \ldots, n\}$, in the limit $h \rightarrow 0$ :

$$
\left\|\left(1-\pi_{h}^{(1)}\right) \tilde{\psi}_{i}\right\|_{H_{w}^{1}}^{2}=O\left(e^{-\frac{2}{h}\left(\max \left[f\left(z_{n}\right)-f\left(z_{i}\right), f\left(z_{i}\right)-f\left(z_{1}\right)\right]+\varepsilon_{1}\right)}\right) .
$$

(b) For any $\delta>0$, in the limit $h \rightarrow 0:\|\nabla \tilde{u}\|_{L_{w}^{2}}^{2}=O\left(e^{-\frac{2}{h}\left(f\left(z_{1}\right)-f\left(x_{0}\right)-\delta\right)}\right)$.

3. There exists $\varepsilon_{0}>0$ such that $\forall(i, j) \in\{1, \ldots, n\}^{2}$ with $i<j$, in the limit $h \rightarrow 0$ :

$$
\left\langle\tilde{\psi}_{i}, \tilde{\psi}_{j}\right\rangle_{L_{w}^{2}}=O\left(e^{-\frac{1}{h}\left(f\left(z_{j}\right)-f\left(z_{i}\right)+\varepsilon_{0}\right)}\right) .
$$

4. (a) There exist constants $\left(C_{i}\right)_{i=1, \ldots, n}$ and $p$ which do not depend on $h$ such that for all $i \in\{1, \ldots, n\}$, in the limit $h \rightarrow 0$ :

$$
\left\langle\nabla \tilde{u}, \tilde{\psi}_{i}\right\rangle_{L_{w}^{2}}=C_{i} h^{p} e^{-\frac{1}{h}\left(f\left(z_{i}\right)-f\left(x_{0}\right)\right)}(1+O(h)) .
$$

(b) There exist constants $\left(B_{i}\right)_{i=1, \ldots, n}$ and $m$ which do not depend on $h$ such that for all $(i, j) \in\{1, \ldots, n\}^{2}$, in the limit $h \rightarrow 0$ :

$$
\int_{\Sigma_{i}} \tilde{\psi}_{j} \cdot n e^{-\frac{2}{h} f} d \sigma= \begin{cases}B_{i} h^{m} e^{-\frac{1}{h} f\left(z_{i}\right)}(1+O(h)) & \text { if } i=j \\ 0 & \text { if } i \neq j .\end{cases}
$$

Then, for all $i \in\{1, \ldots, n\}$, in the limit $h \rightarrow 0$ :

$$
\int_{\Sigma_{i}}\left(\partial_{n} u_{h}\right) e^{-\frac{2}{h} f} d \sigma=C_{i} B_{i} h^{p+m} e^{-\frac{1}{h}\left(2 f\left(z_{i}\right)-f\left(x_{0}\right)\right)}(1+O(h)),
$$

where $u_{h}$ is the eigenfunction associated with the smallest eigenvalue of $-L_{f, h}^{D,(0)}(\Omega)$ (see Proposition 22) which satisfies (11). 
Let us comment on the assumptions made on the quasi-modes. Assumption 1 gives the proper functional setting and the normalization. Assumption 2 will be used to show that $\operatorname{span}\left(\tilde{\psi}_{i}, i=1, \ldots, n\right)$ (respectively $\operatorname{span}(\tilde{u})$ ) is included in $\operatorname{Ran}\left(\pi_{h}^{(1)}\right)$ (respectively in $\left.\operatorname{Ran}\left(\pi_{h}^{(0)}\right)=\operatorname{span}\left(u_{h}\right)\right)$ up to exponentially small terms. Assumption 3 states the quasi-orthonormality of the quasi-modes $\left(\tilde{\psi}_{i}\right)_{i=1, \ldots, n}$. Finally, Assumption 4(a) gives the components of the decomposition of $\nabla \tilde{u}$ over $\operatorname{span}\left(\tilde{\psi}_{i}, i=1, \ldots, n\right)$, and Assumption 4 (b) is then used to find the asymptotic behavior of $\int_{\Sigma_{i}}\left(\partial_{n} u_{h}\right) e^{-\frac{2}{h} f} d \sigma$, knowing those of $\int_{\Sigma_{i}} \tilde{\psi}_{j} \cdot n e^{-\frac{2}{h} f} d \sigma$.

Theorem 1 is a consequence of the existence of quasi-modes satisfying this proposition. The construction of such quasi-modes $\tilde{u}$ and $\left(\tilde{\psi}_{i}\right)_{i=1, \ldots, n}$ satisfying the requirements of Proposition 25 will be the focus of Section 4. where explicit values for the constants $B_{i}$, $C_{i}, p$ and $m$ will be given in (241) and (245). Let us mention that, from (241) and 245), it holds for all $i \in\{1, \ldots, n\}, C_{i} B_{i}<0$, which is consistent with the fact that $\partial_{n} u_{h}<0$ on $\partial \Omega$ (due to the first statement in (11) and the Hopf Lemma, see [28, Section 6.4.2]).

\subsubsection{Assumptions on quasi-modes for $\Delta_{f, h}^{D,(p)}, p \in\{0,1\}$}

The quasi-modes $\left(\tilde{\psi}_{i}\right)_{i \in\{1, \ldots, n\}}$ will be built using eigenforms of some Witten Laplacians. It will thus be more convenient to work in non weighted Sobolev spaces, and to actually consider the 1-forms (see 47): for $i \in\{1, \ldots, n\}$,

$$
\tilde{\phi}_{i}:=e^{-\frac{1}{h} f} \tilde{\psi}_{i} \in \Lambda^{1} H_{T}^{1}(\Omega) .
$$

For further references, let us rewrite the hypotheses on the 1 -forms $\left(\tilde{\psi}_{j}\right)_{j=1, \ldots, n}$ stated in Proposition 25 in terms of the 1 -forms $\left(\tilde{\phi}_{i}\right)_{i=1, \ldots, n}$ defined by (55):

1. For all $i \in\{1, \ldots, n\}, \tilde{\phi}_{i} \in \Lambda^{1} H_{T}^{1}(\Omega)$ and $\left\|\tilde{\phi}_{i}\right\|_{L^{2}}=1$.

2. There exist $\varepsilon_{1}>0$ such that for all $i \in\{1, \ldots, n\}$, in the limit $h \rightarrow 0$ :

$$
\left\|\left(1-\pi_{\left[0, h^{\frac{3}{2}}\right)}\left(\Delta_{f, h}^{D,(1)}(\Omega)\right)\right) \tilde{\phi}_{i}\right\|_{H^{1}}^{2}=O\left(e^{-\frac{2}{h}\left(\max \left[f\left(z_{n}\right)-f\left(z_{i}\right), f\left(z_{i}\right)-f\left(z_{1}\right)\right]+\varepsilon_{1}\right)}\right) .
$$

3. There exists $\varepsilon_{0}>0$ such that $\forall(i, j) \in\{1, \ldots, n\}^{2}, i<j$, in the limit $h \rightarrow 0$ :

$$
\int_{\Omega} \tilde{\phi}_{i}(x) \cdot \tilde{\phi}_{j}(x) d x=O\left(e^{-\frac{1}{h}\left[f\left(z_{j}\right)-f\left(z_{i}\right)+\varepsilon_{0}\right]}\right) .
$$

4. (a) There exist constants $\left(C_{i}\right)_{i=1, \ldots, n}$ and $p$ which do not depend on $h$ such that for all $i \in\{1, \ldots, n\}$, in the limit $h \rightarrow 0$ :

$$
\int_{\Omega} \nabla \tilde{u} \cdot \tilde{\phi}_{i} e^{-\frac{1}{h} f}=C_{i} h^{p} e^{-\frac{1}{h}\left(f\left(z_{i}\right)-f\left(x_{0}\right)\right)}(1+O(h)) .
$$

(b) There exist constants $\left(B_{i}\right)_{i=1, \ldots, n}$ and $m$ which do not depend on $h$ such that for all $(i, j) \in\{1, \ldots, n\}^{2}$, in the limit $h \rightarrow 0$ :

$$
\int_{\Sigma_{i}} \tilde{\phi}_{j} \cdot n e^{-\frac{1}{h} f} d \sigma= \begin{cases}B_{i} h^{m} e^{-\frac{1}{h} f\left(z_{i}\right)}(1+O(h)) & \text { if } i=j \\ 0 & \text { if } i \neq j\end{cases}
$$


As mentioned above, the construction of quasi-modes $\tilde{u}$ and $\left(\tilde{\phi}_{i}\right)_{i=1, \ldots, n}$ satisfying those estimates will be the purpose of Section 4.

Let us comment on the equivalence between the first assumption here (namely (56)) and assumption 2(a) in Proposition 25 (namely (54)). This equivalence is a consequence of the following relation between the projectors which comes from 49):

$$
\pi_{\left[0, h^{\frac{3}{2}}\right)}\left(\Delta_{f, h}^{D,(1)}(\Omega)\right)=e^{-\frac{1}{h} f} \pi_{h}^{(1)} e^{\frac{1}{h} f} .
$$

Indeed, using this relation, one has:

$$
\left\|e^{-\frac{1}{h} f}\left(1-\pi_{h}^{(1)}\right) \tilde{\psi}_{i}\right\|_{H^{1}}=\left\|\left(1-\pi_{\left[0, h^{\frac{3}{2}}\right)}\left(\Delta_{f, h}^{D,(1)}(\Omega)\right)\right) \tilde{\phi}_{i}\right\|_{H^{1}} .
$$

Moreover, one easily checks that there exists $C>0$ such that, for all $h \in(0,1)$ and for all $u \in \Lambda^{p} H^{1}(\Omega)$,

Therefore

$$
\|u\|_{H_{w}^{1}} \leq \frac{C}{h}\left\|u e^{-\frac{1}{h} f}\right\|_{H^{1}} .
$$

$$
\left\|\left(1-\pi_{h}^{(1)}\right) \tilde{\psi}_{i}\right\|_{H_{w}^{1}} \leq \frac{C}{h}\left\|\left(1-\pi_{\left[0, h^{\frac{3}{2}}\right)}\left(\Delta_{f, h}^{D,(1)}(\Omega)\right)\right) \tilde{\phi}_{i}\right\|_{H^{1}}
$$

which shows that (56) (with $\varepsilon_{1}$ ) implies (54) (with $\varepsilon_{1} / 2$ ). A similar reasoning shows that (54) also implies (56), but this will not be used in the following.

\subsection{Proof of Proposition 25}

In all this section, we assume that the hypotheses [H1], [H2] and [H3] hold and we assume the existence of $n+1$ quasi-modes $\left(\tilde{u},\left(\tilde{\psi}_{i}\right)_{i=1, \ldots, n}\right)$ satisfying the conditions of Proposition 25. In the following, $\varepsilon$ denotes a positive constant independent of $h$, smaller than $\min \left(\varepsilon_{1}, \varepsilon_{0}\right)$, and whose precise value may vary (a finite number of times) from one occurrence to the other.

Let us start the proof with two preliminary lemmas relating $\tilde{u}$ with $u_{h}$ on the one hand, and $\operatorname{span}\left(\tilde{\psi}_{j}, j=1, \ldots, n\right)$ with $\operatorname{Ran} \pi_{h}^{(1)}$ on the other hand.

Lemma 26. Let us assume that the assumptions of Proposition 25 hold. Then there exist $c>0$ and $h_{0}>0$ such that for $h \in\left(0, h_{0}\right)$,

$$
\left\|\pi_{h}^{(0)} \tilde{u}\right\|_{L_{w}^{2}}=1+O\left(e^{-\frac{c}{h}}\right) .
$$

Proof. Since $\tilde{u} \in \Lambda^{0} H_{w, T}^{1},\left\|\left(1-\pi_{h}^{(0)}\right) \tilde{u}\right\|_{L_{w}^{2}}$ is bounded from above by $h^{1 / 4}\|\nabla \tilde{u}\|_{L_{w}^{2}}$ thanks to Lemma 23 In addition since $\|\nabla \tilde{u}\|_{L_{w}^{2}}^{2}=O\left(e^{-\frac{2}{h}\left[f\left(z_{1}\right)-f\left(x_{0}\right)-\delta\right]}\right)$ (see assumption 2(b) in Proposition 25), by taking $\delta \in\left(0, f\left(z_{1}\right)-f\left(x_{0}\right)\right)$, one gets that $\left\|\pi_{h}^{(0)} \tilde{u}\right\|_{L_{w}^{2}}=1+O\left(e^{-\frac{c}{h}}\right)$.

As a direct consequence of Lemma 26, one has that for $h$ small enough $\pi_{h}^{(0)} \tilde{u} \neq 0$ and therefore (since moreover $\tilde{u}$ is non negative in $\Omega$ : $\left\langle u_{h}, \pi_{h} \tilde{u}\right\rangle_{L_{w}^{2}}=\left\langle u_{h}, \tilde{u}\right\rangle_{L_{w}^{2}} \geq 0$ ),

$$
u_{h}=\frac{\pi_{h}^{(0)} \tilde{u}}{\left\|\pi_{h}^{(0)} \tilde{u}\right\|_{L_{w}^{2}}} .
$$

Additionally, one has the following lemma concerning the 1-forms. 
Lemma 27. Let us assume that the assumptions of Proposition 25 hold. Then there exists $h_{0}$ such that for all $h \in\left(0, h_{0}\right)$,

$$
\operatorname{span}\left(\pi_{h}^{(1)} \tilde{\psi}_{i}, i=1, \ldots, n\right)=\operatorname{Ran} \pi_{h}^{(1)} .
$$

Proof. The determinant of the Gram matrix of the 1 -forms $\left(\pi_{h}^{(1)} \tilde{\psi}_{i}\right)_{i=1, \ldots, n}$ is $1+$ $O\left(e^{-c / h}\right)$ thanks to the following identity

$$
\left\langle\pi_{h}^{(1)} \tilde{\psi}_{i}, \pi_{h}^{(1)} \tilde{\psi}_{j}\right\rangle_{L_{w}^{2}}=-\left\langle\left(\pi_{h}^{(1)}-1\right) \tilde{\psi}_{i},\left(\pi_{h}^{(1)}-1\right) \tilde{\psi}_{j}\right\rangle_{L_{w}^{2}}+\left\langle\tilde{\psi}_{i}, \tilde{\psi}_{j}\right\rangle_{L_{w}^{2}}
$$

and the fact that, from assumptions 1, 2(a) and 3 in Proposition 25, there exist $h_{0}>0$, $c>0$ such that for $h \in\left(0, h_{0}\right)$,

$$
\left\langle\tilde{\psi}_{i}, \tilde{\psi}_{j}\right\rangle_{L_{w}^{2}}=\left(1-\delta_{i, j}\right) O\left(e^{-\frac{c}{h}}\right)+\delta_{i, j} \text { and }\left\|\left(1-\pi_{h}^{(1)}\right) \tilde{\psi}_{i}\right\|_{H_{w}^{1}}^{2}=O\left(e^{-\frac{c}{h}}\right) .
$$

Moreover, from Proposition 24, $\operatorname{dim} \operatorname{Ran} \pi_{h}^{(1)}=n$. This proves Lemma 27 .

Thanks to Lemma 27, one can build on orthonormal basis $\left(\psi_{i}\right)_{i=1, \ldots, n}$ of $\operatorname{Ran}\left(\pi_{h}^{(1)}\right)$ using a Gram-Schmidt orthonormalization procedure on $\left(\pi_{h}^{(1)}\left(\tilde{\psi}_{i}\right)\right)_{i=1, \ldots, n}$. This will be done in Section 2.3.1 below. Then, since

$$
\nabla u_{h} \in \operatorname{Ran}\left(\pi_{h}^{(1)}\right)=\operatorname{span}\left(\psi_{j}, j=1, \ldots, n\right)
$$

(see (52)) and $\left\|\psi_{j}\right\|_{L_{w}^{2}}=1$, one has

$$
\int_{\Sigma_{k}}\left(\partial_{n} u_{h}\right) e^{-\frac{2}{h} f} d \sigma=\sum_{j=1}^{n}\left\langle\nabla u_{h}, \psi_{j}\right\rangle_{L_{w}^{2}} \int_{\Sigma_{k}} \psi_{j} \cdot n e^{-\frac{2}{h} f} d \sigma .
$$

The proof of Proposition 25 then consists in replacing, in the right-hand side of $(62)$, the function $u_{h}$ by its expression 60 in terms of $\tilde{u}$, and the $\left(\psi_{i}\right)_{i=1, \ldots, n}$ by the $\left(\tilde{\psi}_{i}\right)_{i=1, \ldots, n}$, and to use the assumptions of Proposition 25 to get an asymptotic equivalent of $\int_{\Sigma_{k}}\left(\partial_{n} u_{h}\right) e^{-\frac{2}{h} f} d \sigma$ when $h \rightarrow 0$. In Section 2.3.2. one provides asymptotic equivalents on $\left\langle\nabla u_{h}, \psi_{j}\right\rangle_{L_{w}^{2}}$ for $j \in\{1, \ldots, n\}$. In Section 2.3.3, one gives asymptotic equivalents on $\int_{\Sigma_{k}} \psi_{j} \cdot n e^{-\frac{2}{h} f}$ for $(k, j) \in\{1, \ldots, n\}^{2}$. All these results are then gathered to conclude the proof of Proposition 25 in Section 2.3.4.

\subsubsection{Gram-Schmidt orthonormalization}

Using a Gram-Schmidt procedure, one obtains the following result.

Lemma 28. Let us assume that the assumptions of Proposition 25 hold. Then for all $j \in\{1, \ldots, n\}$, there exist $\left(\kappa_{j i}\right)_{i=1, \ldots, j-1} \in \mathbb{R}^{j-1}$ such that the 1 -forms

$$
v_{j}:=\pi_{h}^{(1)}\left[\tilde{\psi}_{j}+\sum_{i=1}^{j-1} \kappa_{j i} \tilde{\psi}_{i}\right]
$$

(with the convention $\sum_{i=1}^{0}=0$ ) satisfy 
- for all $k \in\{1, \ldots, n\}, \operatorname{span}\left(v_{i}, i=1, \ldots, k\right)=\operatorname{span}\left(\pi_{h}^{(1)} \tilde{\psi}_{i}, i=1, \ldots, k\right)$,

- for all $i \neq j,\left\langle v_{i}, v_{j}\right\rangle_{L_{w}^{2}}=0$.

In the following, we denote by

$$
Z_{j}:=\left\|v_{j}\right\|_{L_{w}^{2}} \text { and } \psi_{j}:=\frac{v_{j}}{Z_{j}}
$$

the normalized 1-forms.

We are first interested in estimating $\kappa_{j i}$ and $Z_{j}$.

Lemma 29. Let us assume that the assumptions of Proposition 25 hold. There exist $\varepsilon>0$ and $h_{0}>0$ such that for all $(i, j) \in\{1, \ldots, n\}^{2}$ with $i<j$ and all $h \in\left(0, h_{0}\right)$

$$
\left\langle\pi_{h}^{(1)} \tilde{\psi}_{i}, \pi_{h}^{(1)} \tilde{\psi}_{j}\right\rangle_{L_{w}^{2}}=O\left(e^{-\frac{1}{h}\left(f\left(z_{j}\right)-f\left(z_{i}\right)+\varepsilon\right)}\right) .
$$

Proof. Using assumption 2(a) in Proposition 25, one gets: for $i<j$,

$$
\begin{aligned}
\left\langle\left(1-\pi_{h}^{(1)}\right) \tilde{\psi}_{j},\left(1-\pi_{h}^{(1)}\right) \tilde{\psi}_{i}\right\rangle_{L_{w}^{2}} & \leq\left\|\left(1-\pi_{h}^{(1)}\right) \tilde{\psi}_{k}\right\|_{H_{w}^{1}}\left\|\left(1-\pi_{h}^{(1)}\right) \tilde{\psi}_{k}\right\|_{H_{w}^{1}} \\
& =O\left(e^{-\frac{1}{h}\left(f\left(z_{n}\right)-f\left(z_{i}\right)+f\left(z_{j}\right)-f\left(z_{1}\right)+\varepsilon\right)}\right) \\
& =O\left(e^{-\frac{1}{h}\left(f\left(z_{j}\right)-f\left(z_{i}\right)+\varepsilon\right)}\right) .
\end{aligned}
$$

The result is then a consequence of assumption 3 in Proposition 25 and the identity (61).

Notice that since $\pi_{h}^{(1)}$ is an $L_{w}^{2}$-projection, $\left\langle\pi_{h}^{(1)} \tilde{\psi}_{i}, \pi_{h}^{(1)} \tilde{\psi}_{j}\right\rangle_{L_{w}^{2}}=\left\langle\pi_{h}^{(1)} \tilde{\psi}_{i}, \tilde{\psi}_{j}\right\rangle_{L_{w}^{2}}$. This will be used extensively in the following.

Lemma 30. Let us assume that the assumptions of Proposition 25 hold. Then there exist $h_{0}>0, \varepsilon>0$ and $c>0$ such that for all $j \in\{1, \ldots, n\}, i \in\{1, \ldots, j-1\}$ and $h \in\left(0, h_{0}\right)$

$$
\kappa_{j i}=O\left(e^{-\frac{1}{h}\left(f\left(z_{j}\right)-f\left(z_{i}\right)+\varepsilon\right)}\right) \text { and } Z_{j}=1+O\left(e^{-\frac{c}{h}}\right) .
$$

Proof. Let us introduce the notation: for all $i \in\{1, \ldots, n\}$,

$$
r_{i}:=\left\|\left(1-\pi_{h}^{(1)}\right) \tilde{\psi}_{i}\right\|_{H_{w}^{1}}^{2} .
$$

Let us prove this lemma by induction. Concerning $\psi_{1}$, one has from Lemma 28

$$
\psi_{1}=\frac{v_{1}}{Z_{1}} \text { with } v_{1}=\pi_{h}^{(1)} \tilde{\psi}_{1} .
$$

Since $\left\|\tilde{\psi}_{1}\right\|_{L_{w}^{2}}=1$, one has $Z_{1}=\left\|\pi_{h}^{(1)} \tilde{\psi}_{1}\right\|_{L_{w}^{2}} \leq 1$. In addition, by Pythagorean Theorem and by assumption 2(a) in Proposition 25 on $r_{1}$, there exists $c>0$ such that for $h$ small enough

$$
Z_{1}^{2} \geq 1-\left\|\left(1-\pi_{h}^{(1)}\right) \tilde{\psi}_{1}\right\|_{L_{w}^{2}}^{2} \geq 1-r_{1} \geq 1-e^{-\frac{c}{h}}
$$


Thus $Z_{1}=1+O\left(e^{-\frac{c}{h}}\right)$. Now, concerning $\psi_{2}$, one has

$$
\psi_{2}=\frac{v_{2}}{Z_{2}} \text { with } v_{2}=\pi_{h}^{(1)} \tilde{\psi}_{2}-\left\langle\pi_{h}^{(1)} \tilde{\psi}_{2}, \psi_{1}\right\rangle_{L_{w}^{2}} \psi_{1},
$$

and thus $\kappa_{21}=-\frac{1}{Z_{1}^{2}}\left\langle\pi_{h}^{(1)} \tilde{\psi}_{1}, \tilde{\psi}_{2}\right\rangle_{L_{w}^{2}}=O\left(e^{-\frac{1}{h}\left(f\left(z_{2}\right)-f\left(z_{1}\right)+\varepsilon\right)}\right)$ (by Lemma 29. Then one obtains that $Z_{2}=1+O\left(e^{-\frac{c}{h}}\right)$ by a similar reasoning as the one we used above for $Z_{1}$.

In order to prove Lemma 30 by induction, let us now assume that for $k \in\{1, \ldots, n\}$ and for all $j \in\{1, \ldots, k\}, i \in\{1, \ldots, j-1\}$,

$$
\kappa_{j i}=O\left(e^{-\frac{1}{h}\left[f\left(z_{j}\right)-f\left(z_{i}\right)+\varepsilon\right]}\right) \text { and } Z_{j}=1+O\left(e^{-\frac{c}{h}}\right) .
$$

One gets by the Gram-Schmidt procedure which defines the $\left(\psi_{i}\right)_{i=1, \ldots, n}$,

$$
\psi_{k+1}=\frac{v_{k+1}}{Z_{k+1}}
$$

where, using the notation $\kappa_{i i}=1$,

$$
\begin{aligned}
v_{k+1} & =\pi_{h}^{(1)} \tilde{\psi}_{k+1}-\sum_{j=1}^{k}\left\langle\pi_{h}^{(1)} \tilde{\psi}_{k+1}, \psi_{j}\right\rangle_{L_{w}^{2}} \psi_{j} \\
& =\pi_{h}^{(1)} \tilde{\psi}_{k+1}-\sum_{j=1}^{k} \sum_{l, q=1}^{j} \frac{1}{Z_{j}^{2}}\left\langle\pi_{h}^{(1)} \tilde{\psi}_{k+1}, \pi_{h}^{(1)} \tilde{\psi}_{l}\right\rangle_{L_{w}^{2}} \kappa_{j l} \kappa_{j q} \pi_{h}^{(1)} \tilde{\psi}_{q} \\
& =\pi_{h}^{(1)} \tilde{\psi}_{k+1}-\sum_{q=1}^{k} \pi_{h}^{(1)} \tilde{\psi}_{q} \sum_{j=q}^{k} \sum_{l=1}^{j} \frac{1}{Z_{j}^{2}}\left\langle\pi_{h}^{(1)} \tilde{\psi}_{k+1}, \pi_{h}^{(1)} \tilde{\psi}_{l}\right\rangle_{L_{w}^{2}} \kappa_{j l} \kappa_{j q} .
\end{aligned}
$$

Then for $q \in\{1, \ldots, k\}$,

$$
\kappa_{(k+1) q}=-\sum_{j=q}^{k} \sum_{l=1}^{j} \frac{1}{Z_{j}^{2}}\left\langle\pi_{h}^{(1)} \tilde{\psi}_{k+1}, \pi_{h}^{(1)} \tilde{\psi}_{l}\right\rangle_{L_{w}^{2}} \kappa_{j l} \kappa_{j q} .
$$

Since $Z_{j}=1+O\left(e^{-\frac{c}{h}}\right)$, one gets $Z_{j}^{-1}=1+O\left(e^{-\frac{c}{h}}\right)$. Using Lemma 29 , one obtains $\left\langle\pi_{h}^{(1)} \tilde{\psi}_{k+1}, \pi_{h}^{(1)} \tilde{\psi}_{l}\right\rangle_{L_{w}^{2}}=O\left(e^{-\frac{1}{h}\left[f\left(z_{k+1}\right)-f\left(z_{l}\right)+\varepsilon\right]}\right)$, since $l<k+1$. Moreover, since $l \leq j$ and $q \leq j$, it holds:

$$
\kappa_{j l}=O\left(e^{-\frac{1}{h}\left[f\left(z_{j}\right)-f\left(z_{l}\right)\right]}\right) \text { and } \kappa_{j q}=O\left(e^{-\frac{1}{h}\left[f\left(z_{j}\right)-f\left(z_{q}\right)\right]}\right) .
$$

Consequently, one obtains that

$$
\frac{1}{Z_{j}^{2}}\left\langle\pi_{h}^{(1)} \tilde{\psi}_{k+1}, \pi_{h}^{(1)} \tilde{\psi}_{l}\right\rangle_{L_{w}^{2}} \kappa_{j l} \kappa_{j q}=O\left(e^{-\frac{1}{h}\left[f\left(z_{k+1}\right)-f\left(z_{q}\right)+2\left(f\left(z_{j}\right)-f\left(z_{l}\right)\right)+\varepsilon\right]}\right) .
$$

Thus, since $f\left(z_{j}\right) \geq f\left(z_{l}\right)$, one obtains from 65$)$, that for $q \in\{1, \ldots, k\}$, there exists $\varepsilon>0$ such that for $h$ small enough:

$$
\kappa_{(k+1) q}=O\left(e^{-\frac{1}{h}\left[f\left(z_{k+1}\right)-f\left(z_{q}\right)+\varepsilon\right]}\right) .
$$

The estimate $Z_{k+1}=1+O\left(e^{-\frac{c}{h}}\right)$ is a consequence of the fact that $\left(\kappa_{(k+1) q}\right)_{q \in\{1, \ldots, k\}}$ are exponentially small together with the estimate

$$
\left\|\pi_{h}^{(1)} \tilde{\psi}_{k+1}\right\|_{L_{w}^{2}}=1+O\left(e^{-\frac{c}{h}}\right) .
$$

This concludes the proof of Lemma 30 by induction. 


\subsubsection{Estimates on the interaction terms $\left(\left\langle\nabla u_{h}, \psi_{j}\right\rangle_{L_{w}^{2}}\right)_{j \in\{1, \ldots, n\}}$}

Lemma 31. Let us assume that the assumptions of Proposition 25 hold. Then for $j \in\{1, \ldots, n\}$, one has

$$
\left\langle\nabla u_{h}, \psi_{j}\right\rangle_{L_{w}^{2}}=C_{j} h^{p} e^{-\frac{1}{h}\left(f\left(z_{j}\right)-f\left(x_{0}\right)\right)}(1+O(h)) .
$$

Proof. From (50), for any $\phi \in H_{w, T}^{1}(\Omega)$ and $v \in L_{w}^{2}(\Omega)$, it holds,

$$
\left\langle\nabla \pi_{h}^{(0)} \phi, \pi_{h}^{(1)} v\right\rangle_{L_{w}^{2}}=\left\langle\nabla \phi, \pi_{h}^{(1)} v\right\rangle_{L_{w}^{2}} .
$$

Using 60 -63 - 64 - 66 , for all $j \in\{1, \ldots, n\}$, one has

$$
\begin{aligned}
\left\langle\nabla u_{h}, \psi_{j}\right\rangle_{L_{w}^{2}}= & \frac{Z_{j}^{-1}}{\left\|\pi_{h}^{(0)} \tilde{u}\right\|_{L_{w}^{2}}}\left[\left\langle\nabla \tilde{u}, \pi_{h}^{(1)} \tilde{\psi}_{j}\right\rangle_{L_{w}^{2}}+\sum_{i=1}^{j-1} \kappa_{j i}\left\langle\nabla \tilde{u}, \pi_{h}^{(1)} \tilde{\psi}_{i}\right\rangle_{L_{w}^{2}}\right] \\
= & \frac{Z_{j}^{-1}}{\left\|\pi_{h}^{(0)} \tilde{u}\right\|_{L_{w}^{2}}}\left[\left\langle\nabla \tilde{u}, \tilde{\psi}_{j}\right\rangle_{L_{w}^{2}}+\left\langle\nabla \tilde{u},\left(\pi_{h}^{(1)}-1\right) \tilde{\psi}_{j}\right\rangle_{L_{w}^{2}}\right] \\
& +\frac{Z_{j}^{-1}}{\left\|\pi_{h}^{(0)} \tilde{u}\right\|_{L_{w}^{2}}}\left[\sum_{i=1}^{j-1} \kappa_{j i}\left(\left\langle\nabla \tilde{u}, \tilde{\psi}_{i}\right\rangle_{L_{w}^{2}}+\left\langle\nabla \tilde{u},\left(\pi_{h}^{(1)}-1\right) \tilde{\psi}_{i}\right\rangle_{L_{w}^{2}}\right)\right] .
\end{aligned}
$$

From Lemmata 26 and 30 , one has $\frac{Z_{j}^{-1}}{\left\|\pi_{h}^{(0)} \tilde{u}\right\|_{L_{w}^{2}}}=1+O\left(e^{-\frac{c}{h}}\right)$. Using assumptions 2 and 4(a) in Proposition 25 and Lemma 30 , there exists $\delta_{0}>0$ such that for all $\delta \in\left(0, \delta_{0}\right)$,

$$
\begin{aligned}
\left\langle\nabla u_{h}, \psi_{j}\right\rangle_{L_{w}^{2}}= & C_{j} h^{p} e^{-\frac{1}{h}\left[f\left(z_{j}\right)-f\left(x_{0}\right)\right]}(1+O(h)) \\
& +O\left(e^{-\frac{1}{h}\left[f\left(z_{1}\right)-f\left(x_{0}\right)-\delta+f\left(z_{j}\right)-f\left(z_{1}\right)+\varepsilon\right]}\right) \\
& +\sum_{i=1}^{j-1} O\left(e^{-\frac{1}{h}\left[f\left(z_{j}\right)-f\left(z_{i}\right)+\varepsilon+f\left(z_{i}\right)-f\left(x_{0}\right)-\delta\right]}\right) \\
& +\sum_{i=1}^{j-1} O\left(e^{-\frac{1}{h}\left[f\left(z_{j}\right)-f\left(z_{i}\right)+\varepsilon+f\left(z_{1}\right)-f\left(x_{0}\right)-\delta+f\left(z_{i}\right)-f\left(z_{1}\right)+\varepsilon\right]}\right) .
\end{aligned}
$$

Therefore choosing $\delta<\varepsilon$, there exists $\varepsilon^{\prime}>0$ such that

$$
\left\langle\nabla u_{h}, \psi_{j}\right\rangle_{L_{w}^{2}}=C_{j} h^{p} e^{-\frac{1}{h}\left[f\left(z_{j}\right)-f\left(x_{0}\right)\right]}(1+O(h))+O\left(e^{-\frac{1}{h}\left[f\left(z_{j}\right)-f\left(x_{0}\right)+\varepsilon^{\prime}\right]}\right) .
$$

This concludes the proof of Lemma 31 .

\subsubsection{Estimates on the boundary terms $\left(\int_{\Sigma_{k}} \psi_{j} \cdot n e^{-\frac{2}{h} f} d \sigma\right)_{(j, k) \in\{1, \ldots, n\}^{2}}$}

One denotes in this section, for $k \in\{1, \ldots, n\}, K_{k}:=\max \left(f\left(z_{n}\right)-f\left(z_{k}\right), f\left(z_{k}\right)-f\left(z_{1}\right)\right) \geq$ 0 .

Lemma 32. Let us assume that the assumptions of Proposition 25 hold. Then for all $(j, k) \in\{1, \ldots, n\}^{2}$, there exists $\varepsilon>0$ such that it holds

$$
\int_{\Sigma_{k}} \psi_{j} \cdot n e^{-\frac{2}{h} f} d \sigma= \begin{cases}O\left(e^{-\frac{1}{h}\left[f\left(z_{j}\right)+\varepsilon\right]}\right) & \text { if } k<j, \\ B_{j} h^{m} e^{-\frac{1}{h} f\left(z_{j}\right)}(1+O(h)) & \text { if } k=j, \\ O\left(e^{-\frac{1}{h}\left[K_{j}+f\left(z_{k}\right)+\varepsilon\right]}\right)+\sum_{i=1}^{j-1} O\left(e^{-\frac{1}{h}\left[f\left(z_{j}\right)-f\left(z_{i}\right)+K_{i}+f\left(z_{k}\right)+\varepsilon\right]}\right) & \text { if } k>j .\end{cases}
$$


Proof. Using 63)-64 and writing $\pi_{h}^{(1)} \tilde{\psi}_{i}=\tilde{\psi}_{i}+\left(\pi_{h}^{(1)}-1\right) \tilde{\psi}_{i}$, one obtains that

$$
\int_{\Sigma_{k}} \psi_{j} \cdot n e^{-\frac{2}{h} f} d \sigma=a_{j k}+b_{j k}+\sum_{i=1}^{j-1}\left(c_{j k i}+d_{j k i}\right)
$$

with for $(j, k) \in\{1, \ldots, n\}^{2}$ and $i \in\{1, \ldots, j-1\}$,

$$
\begin{gathered}
a_{j k}=Z_{j}^{-1} \int_{\Sigma_{k}} \tilde{\psi}_{j} \cdot n e^{-\frac{2}{h} f} d \sigma, b_{j k}=Z_{j}^{-1} \int_{\Sigma_{k}}\left(\pi_{h}^{(1)}-1\right) \tilde{\psi}_{j} \cdot n e^{-\frac{2}{h} f} d \sigma \\
c_{j k i}=Z_{j}^{-1} \kappa_{j i} \int_{\Sigma_{k}} \tilde{\psi}_{i} \cdot n e^{-\frac{2}{h} f} d \sigma \text { and } d_{j k i}=Z_{j}^{-1} \kappa_{j i} \int_{\Sigma_{k}}\left(\pi_{h}^{(1)}-1\right) \tilde{\psi}_{i} \cdot n e^{-\frac{2}{h} f} d \sigma .
\end{gathered}
$$

Using the trace theorem and assumption 2(a) in Proposition 25, one has, for some universal constant $C$,

$$
\begin{aligned}
\int_{\Sigma_{k}}\left(\pi_{h}^{(1)}-1\right) \tilde{\psi}_{j} \cdot n e^{-\frac{2}{h} f} d \sigma & \leq \frac{C}{h}\left\|\left(\pi_{h}^{(1)}-1\right) \tilde{\psi}_{j}\right\|_{H_{w}^{1}} \sqrt{\int_{\Sigma_{k}} e^{-\frac{2}{h} f}} \\
& =O\left(e^{-\frac{1}{h}\left[K_{j}+f\left(z_{k}\right)+\varepsilon\right]}\right) .
\end{aligned}
$$

If $k=j$ and $i \in\{1, \ldots, j-1\}$, one gets, using (68), Lemma 30 and assumption 4(b) in Proposition 25.

$$
\begin{gathered}
a_{j k}=B_{j} h^{m} e^{-\frac{1}{h} f\left(z_{j}\right)}(1+O(h)), b_{j k}=O\left(e^{-\frac{1}{h}\left[K_{j}+f\left(z_{j}\right)+\varepsilon\right]}\right), \\
c_{j k i}=0 \text { and } d_{j k i}=O\left(e^{-\frac{1}{h}\left[f\left(z_{j}\right)-f\left(z_{i}\right)+K_{i}+f\left(z_{j}\right)+\varepsilon\right]}\right) .
\end{gathered}
$$

If $k \neq j$ and $i \in\{1, \ldots, j-1\}$, one gets using again (68), Lemma 30 and assumption 4(b) in Proposition 25:

$$
\begin{gathered}
a_{j k}=0, b_{j k}=O\left(e^{-\frac{1}{h}\left[K_{j}+f\left(z_{k}\right)+\varepsilon\right]}\right), \\
c_{j k i}=\left\{\begin{array}{ll}
O\left(e^{-\frac{1}{h}\left[f\left(z_{j}\right)+\varepsilon\right]}\right) & \text { if } k=i, \\
0 & \text { if } k \neq i,
\end{array} \text { and } d_{j k i}=O\left(e^{-\frac{1}{h}\left[f\left(z_{j}\right)-f\left(z_{i}\right)+K_{i}+f\left(z_{k}\right)+\varepsilon\right]}\right) .\right.
\end{gathered}
$$

Notice that $c_{j k i}=0$ if $j<k$ and that if $j>k$ there exists only one $i$ such that $c_{j k i} \neq 0$, namely $i=k$. This concludes the proof of the Lemma 32 .

\subsubsection{Estimates on $\left(\int_{\Sigma_{k}}\left(\partial_{n} u_{h}\right) e^{-\frac{2}{h} f} d \sigma\right)_{k \in\{1, \ldots, n\}}$}

We are now in position to conclude the proof of Proposition 25, by proving that for $k \in\{1, \ldots, n\}$, one has

$$
\int_{\Sigma_{k}}\left(\partial_{n} u_{h}\right) e^{-\frac{2}{h} f} d \sigma=C_{k} B_{k} h^{p+m} e^{-\frac{1}{h}\left(2 f\left(z_{k}\right)-f\left(x_{0}\right)\right)}(1+O(h)) .
$$

Proof. Let us assume that the assumptions of Proposition 25 hold. Let us recall the decomposition 62):

$$
\int_{\Sigma_{k}}\left(\partial_{n} u_{h}\right) e^{-\frac{2}{h} f} d \sigma=\sum_{j=1}^{n}\left\langle\nabla u_{h}, \psi_{j}\right\rangle_{L_{w}^{2}} \int_{\Sigma_{k}} \psi_{j} \cdot n e^{-\frac{2}{h} f} d \sigma
$$


Using Lemmas 31 and 32 , we can now estimate the terms in the sum in the right-hand side. If $j>k$, there exist $\varepsilon>0$ and $h_{0}>0$ such that for all $h \in\left(0, h_{0}\right)$

$$
\begin{aligned}
\left\langle\nabla u_{h}, \psi_{j}\right\rangle_{L_{w}^{2}} \int_{\Sigma_{k}} \psi_{j} \cdot n e^{-\frac{2}{h} f} d \sigma & =C_{j} h^{p} O\left(e^{-\frac{1}{h}\left[f\left(z_{j}\right)-f\left(x_{0}\right)\right]} e^{-\frac{1}{h}\left[f\left(z_{j}\right)+\varepsilon\right]}\right) \\
& =C_{j} h^{p} O\left(e^{-\frac{1}{h}\left[2 f\left(z_{j}\right)-f\left(x_{0}\right)+\varepsilon\right]}\right) \\
& =C_{j} h^{p} O\left(e^{-\frac{1}{h}\left[2 f\left(z_{k}\right)-f\left(x_{0}\right)+\varepsilon\right]}\right) .
\end{aligned}
$$

If $j<k$, there exist $\varepsilon>0$ and $h_{0}>0$ such that for all $h \in\left(0, h_{0}\right)$

$$
\begin{aligned}
& \left\langle\nabla u_{h}, \psi_{j}\right\rangle_{L_{w}^{2}} \int_{\Sigma_{k}} \psi_{j} \cdot n e^{-\frac{2}{h} f} d \sigma \\
& =O\left(e^{-\frac{1}{h}\left[f\left(z_{j}\right)-f\left(x_{0}\right)+K_{j}+f\left(z_{k}\right)+\varepsilon\right]}\right)+\sum_{i=1}^{j-1} O\left(e^{-\frac{1}{h}\left[f\left(z_{j}\right)-f\left(x_{0}\right)+f\left(z_{j}\right)-f\left(z_{i}\right)+K_{i}+f\left(z_{k}\right)+\varepsilon\right]}\right) \\
& =O\left(e^{-\frac{1}{h}\left[f\left(z_{j}\right)-f\left(x_{0}\right)+f\left(z_{n}\right)-f\left(z_{j}\right)+f\left(z_{k}\right)+\varepsilon\right]}\right)+\sum_{i=1}^{j-1} O\left(e^{-\frac{1}{h}\left[f\left(z_{j}\right)-f\left(x_{0}\right)+f\left(z_{j}\right)-f\left(z_{i}\right)+f\left(z_{n}\right)-f\left(z_{i}\right)+f\left(z_{k}\right)+\varepsilon\right]}\right) \\
& =O\left(e^{-\frac{1}{h}\left[f\left(z_{n}\right)+f\left(z_{k}\right)-f\left(x_{0}\right)+\varepsilon\right]}\right)+\sum_{i=1}^{j-1} O\left(e^{-\frac{1}{h}\left[f\left(z_{n}\right)+f\left(z_{k}\right)-f\left(x_{0}\right)+2\left(f\left(z_{j}\right)-f\left(z_{i}\right)\right)+\varepsilon\right]}\right) \\
& =O\left(e^{-\frac{1}{h}\left[2 f\left(z_{k}\right)-f\left(x_{0}\right)+\varepsilon\right]}\right) .
\end{aligned}
$$

Finally if $j=k, \exists \varepsilon>0$ and $\exists h_{0}>0$ such that for all $h \in\left(0, h_{0}\right)$

$$
\left\langle\nabla u_{h}, \psi_{k}\right\rangle_{L_{w}^{2}} \int_{\Sigma_{k}} \psi_{k} \cdot n e^{-\frac{2}{h} f} d \sigma=C_{k} B_{k} h^{p+m} e^{-\frac{1}{h}\left(2 f\left(z_{k}\right)-f\left(x_{0}\right)\right)}(1+O(h)) .
$$

From these estimates, for a fixed $k \in\{1, \ldots, n\}$, the dominant term in the sum in the right-hand side of $(62)$ is the one with index $j=k$, namely (69). This concludes the proof of Proposition 25.

\section{On the Agmon distance}

In this section, we present the main properties of the Agmon distance introduced in Definition 8. The Agmon distance is useful to quantify the decay of eigenforms of Witten Laplacians away from critical points of $f$ and $\left.f\right|_{\partial \Omega}$. The Agmon distance on a domain without boundary has been extensively analyzed in many previous works (see in particular [40 42, 44]). The aim of this section is to generalize well-known results in the case without boundary to our context, namely for bounded domains. Indeed, to the best of our knowledge, this has not been done in the literature before in a comprehensive way.

For simplicity, all the proofs in this section are made for a bounded connected open connected $C^{\infty}$ domain $\Omega \subset \mathbb{R}^{d}$ (equipped with the geodesic Euclidean distance (84)) and for a $C^{\infty}$ function $f: \bar{\Omega} \rightarrow \mathbb{R}$. The generalization to a $C^{\infty}$ compact connected Riemannian manifold of dimension $d$ with boundary is straightforward. The notation $|x-y|$ denotes the Euclidean distance between $x$ and $y$ in $\mathbb{R}^{d}$. If one deals with a compact connected Riemannian manifold of dimension $d$ with boundary, this distance 
has to be replaced by the geodesic distance on $\bar{\Omega}$ for the initial metric and the scalar product between two vectors of $\mathbb{R}^{d}$ has to be replaced by the one induced by the initial metric on the tangent space of $\bar{\Omega}$.

This section is organized as follows. Section 3.1 is devoted to an equivalent definition of the Agmon distance, which will be crucial in the following. In Section 3.2 , we then give a few useful properties of the Agmon distance. As already mentioned in Section 1.5.1. there is a link between the Agmon distance and the eikonal equation. This is explained in Sections 3.3 and 3.5. This link is useful in order to build explicit curves realizing the Agmon distance, as explained in Section 3.4 .

\subsection{The set $A(x, y)$ and an equivalent definition of the Agmon distance}

In order to study the Agmon distance, it will be more convenient for technical reasons to restrict the class of curves appearing in the definition of the Agmon distance (see Definition 8).

Definition 33. For $x, y \in \bar{\Omega}$, we denote by $A(x, y)$ the set of curves $\gamma:[0,1] \rightarrow \bar{\Omega}$ which are Lipschitz with $\gamma(0)=x, \gamma(1)=y$ and such that the set $\partial\{t \in[0,1] \mid \gamma(t) \in \partial \Omega\}$ is finite.

Here, $\partial\{t \in[0,1], \gamma(t) \in \partial \Omega\}$ denotes the boundary of the set $\{t \in[0,1], \gamma(t) \in \partial \Omega\}$. The main result of this section is that, under assumption [H3], the Agmon distance $d_{a}$ satisfies (compare with (18)):

$$
\forall(x, y) \in \bar{\Omega} \times \bar{\Omega}, d_{a}(x, y)=\inf _{\gamma \in A(x, y)} L(\gamma,(0,1)) .
$$

See Corollary 39 below.

The following lemma will be needed several times throughout this section. It motivates the use of the set $A(x, y)$ appearing in Definition 33

Lemma 34. Let $x, y \in \bar{\Omega}$ and $\gamma \in A(x, y)$. Then for any $h: \bar{\Omega} \rightarrow \mathbb{R}$ which is $C^{1}$, one gets

$$
h(y)-h(x)=\int_{\{t \in[0,1], \gamma(t) \in \Omega\}}(\nabla h)(\gamma) \cdot \gamma^{\prime}+\int_{\operatorname{int}\{t \in[0,1], \gamma(t) \in \partial \Omega\}}\left(\nabla_{T} h\right)(\gamma) \cdot \gamma^{\prime} .
$$

Here, the notation int stands for the interior of a domain.

Proof. Since $\gamma$ is Lipschitz, $h \circ \gamma$ is Lipchitz and thus absolutely continuous. Therefore, one has:

$$
\begin{aligned}
h(y)-h(x)= & \int_{0}^{1} \frac{d}{d t}(h \circ \gamma) \\
= & \int_{\{t \in[0,1], \gamma(t) \in \Omega\}} \frac{d}{d t}(h \circ \gamma)+\int_{\operatorname{int}\{t \in[0,1], \gamma(t) \in \partial \Omega\}} \frac{d}{d t}(h \circ \gamma) \\
& +\int_{\partial\{t \in[0,1], \gamma(t) \in \partial \Omega\}} \frac{d}{d t}(h \circ \gamma) .
\end{aligned}
$$

By definition of the set $A(x, y)$ (see Definition 33 the set $\partial\{t \in[0,1], \gamma(t) \in \partial \Omega\}$ has Lebesgue measure zero, and thus $\int_{\partial\{t \in[0,1], \gamma(t) \in \partial \Omega\}} \frac{d}{d t}(h \circ \gamma)=0$. The curve $\gamma$ is continuous and thus the set $\{t \in[0,1], \gamma(t) \in \Omega\}$ is open in $[0,1]$. As a consequence, 
using in addition that since $\gamma$ is Lipschitz, it is almost everywhere differentiable (by the Rademacher Theorem), one has for almost every $t \in[0,1]$ :

$$
\frac{d}{d t} h(\gamma)(t)=\left\{\begin{array}{r}
(\nabla h)(\gamma(t)) \cdot \frac{d}{d t} \gamma(t) \text { a.e. on }\{t \in[0,1], \gamma(t) \in \Omega\} \\
\left(\nabla_{T} h\right)(\gamma(t)) \cdot \frac{d}{d t} \gamma(t) \text { a.e. on } \operatorname{int}\{t \in[0,1], \gamma(t) \in \partial \Omega\}
\end{array}\right.
$$

This proves (71).

Remark 35. Notice that there exist Lipschitz curves $\gamma$ such that $\partial\{t \in[0,1], \gamma(t) \in \partial \Omega\}$ has a positive Lebesgue measure. Let us give an example. Consider $\Omega=(0,1) \times(0,2)$ and the curve

$$
\gamma: t \in[0,1] \mapsto\left(t, \inf _{y \in K}|t-y|\right) \in[0,1]^{2},
$$

where $K$ is the Smith-Volterra-Cantor set in $[0,1]$, such that $K$ is closed, has a positive Lebesgue measure and has an empty interior (see [69, Section 2.5]). Notice that the distance $\inf _{y \in K}|t-y|$ to $K$ is a Lipschitz function of $t \in(0,1)$, so that $\gamma$ is a Lipschitz function. The set $K$ is closed and thus

$$
\{t \in[0,1], \gamma(t) \in \partial \Omega\}=\{t \in[0,1], \gamma(t)=0\}=K .
$$

Therefore $\partial\{t \in[0,1], \gamma(t) \in \partial \Omega\}=\bar{K} \backslash(\operatorname{int} K)=K$.

The following results will be useful to prove the equality 70 and to prove the existence of curves realizing the Agmon distance in Section 3.4 .

Proposition 36. Assume that [H3] holds. Let $\gamma:[0,1] \rightarrow \bar{\Omega}$ be a Lipschitz curve and assume that there exists a time $t^{*} \in[0,1]$ such that $\gamma\left(t^{*}\right) \in \partial \Omega$. Then there exists $(a, b) \in[0,1]^{2}$, with $a \leq t^{*} \leq b$ and $a<b$ such that for all $\left(t_{1}, t_{2}\right) \in[0,1]^{2}$, with $a \leq t_{1}<t_{2} \leq b$, there exists a Lipschitz curve $\gamma_{12}:\left[t_{1}, t_{2}\right] \rightarrow \bar{\Omega}$ satisfying

$$
\begin{gathered}
\gamma_{12}\left(t_{1}\right)=\gamma\left(t_{1}\right) \text { and } \gamma_{12}\left(t_{2}\right)=\gamma\left(t_{2}\right), \\
L\left(\gamma,\left(t_{1}, t_{2}\right)\right) \geq L\left(\gamma_{12},\left(t_{1}, t_{2}\right)\right)
\end{gathered}
$$

and, either $\left\{t \in\left[t_{1}, t_{2}\right], \gamma_{12}(t) \in \partial \Omega\right\}$ is empty, or its boundary $\partial\left\{t \in\left[t_{1}, t_{2}\right], \gamma_{12}(t) \in \partial \Omega\right\}$ consists of isolated points in $\left\{t \in\left[t_{1}, t_{2}\right], \gamma_{12}(t) \in \partial \Omega\right\}$. Moreover, if the following is satisfied:

$$
\exists\left(s_{1}, s_{2}, s_{3}\right) \in\left[t_{1}, t_{2}\right]^{3}, s_{1}<s_{2}<s_{3}, \gamma\left(s_{1}\right) \in \partial \Omega, \gamma\left(s_{2}\right) \in \Omega \text { and } \gamma\left(s_{3}\right) \in \partial \Omega,
$$

then the inequality $(72)$ is strict.

Remark 37. Notice that if $t^{*} \in \partial\{t \in[0,1], \gamma(t) \in \partial \Omega\}$ is not isolated in $\partial\{t \in[0,1], \gamma(t) \in \partial \Omega\}$, then there exists a neighborhood $\left[t_{1}, t_{2}\right]$ of $t^{*}$ in $[0,1]$ such that (73) is satisfied and thus the inequality (72) is strict. Therefore if a Lipschitz curve $\gamma$ realizes the infimum of $L$ on $\operatorname{Lip}(x, y)$, then $\partial\{t \in[0,1], \gamma(t) \in \partial \Omega\}$ is finite. This motivates the introduction of the set $A(x, y)$. 
Proof. Let $t^{*} \in[0,1]$ be such that $\gamma\left(t^{*}\right) \in \partial \Omega$. The proof is divided into three steps.

Step 1: Introduction of a normal system of coordinates and definition of $a$ and $b$.

Let us consider a neighborhood $V_{\partial \Omega}$ of $\gamma\left(t^{*}\right)$ in $\partial \Omega$, and a smooth local system of coordinates in $V_{\partial \Omega} \subset \partial \Omega$, denoted by $x_{T}: V_{\partial \Omega} \rightarrow \mathbb{R}^{d-1}$. Let us now extend it to a tangential and normal system of coordinates around $\gamma\left(t^{*}\right)$ in $\bar{\Omega}$, denoted by $\phi(x)=$ $\left(x_{T}, x_{N}\right)$. The function $\phi$ is defined from a neighborhood of $\gamma\left(t^{*}\right)$ in $\bar{\Omega}$ to $\mathbb{R}^{d}$. Moreover, one has $x_{N}(x) \geq 0$ and for all $x, x_{N}(x)=0$ if and only if $x \in \partial \Omega$. One can assume without loss of generality that $\phi$ is defined on a neighborhood $V_{\alpha}$ of $\gamma\left(t^{*}\right)$ in $\bar{\Omega}$ such that $\phi\left(V_{\alpha}\right)=U \times[0, \alpha]$ for $\alpha>0$, and $U \subset \mathbb{R}^{d-1}$. For this normal system of coordinates, the metric tensor $G$ is such that: $\forall\left(x_{T}, x_{N}\right) \in U \times[0, \alpha]$,

$$
G\left(x_{T}, x_{N}\right)=\left(\begin{array}{cc}
\tilde{G}\left(x_{T}, x_{N}\right) & 0 \\
0 & G_{N N}\left(x_{T}, x_{N}\right)
\end{array}\right),
$$

where $\tilde{G}\left(x_{T}, x_{N}\right) \in \mathbb{R}^{(d-1) \times(d-1)}$ and $G_{N N}\left(x_{T}, x_{N}\right) \in \mathbb{R}$ are smooth functions of $\left(x_{T}, x_{N}\right)$. The existence of such a system of coordinates is a consequence of the collar theorem, see 74 .

Under assumption [H3] (namely $\partial_{n} f>0$ on $\partial \Omega$ ), there exist constants $A>1$ and $\varepsilon_{1}>0$ such that for all $x_{N} \in\left(0, \varepsilon_{1}\right]$ and for all $x_{T} \in U$, (see (17) for the definition of $g$ )

$$
g\left(\phi^{-1}\left(x_{T}, x_{N}\right)\right) \geq A g\left(\phi^{-1}\left(x_{T}, 0\right)\right) .
$$

Since the local change of coordinates is smooth, for all $\varepsilon \in(0,1)$, there exists $\eta>0$ such that for all $x_{N} \in[0, \eta]$ and for all $x_{T} \in U$, one has

$$
\tilde{G}\left(x_{T}, x_{N}\right) \geq(1-\varepsilon)^{2} \tilde{G}\left(x_{T}, 0\right) .
$$

Let us choose $\varepsilon>0$ such that $(1-\varepsilon) A>1$. One can reduce $V_{\alpha}$ such that $0 \leq x_{N}(x) \leq$ $\min \left(\eta, \varepsilon_{1}\right)$ for all $x \in V_{\alpha}$. By continuity of $\gamma$, there exist $(a, b) \in[0,1]^{2}$, with $a \leq t^{*} \leq b$ and $a<b$ such that for all $t \in[a, b], \gamma(t) \in V_{\alpha}$. Let us introduce the components of $\gamma$ in the normal system of coordinates: $\left(\gamma_{T}(t), \gamma_{N}(t)\right)=\phi(\gamma(t))$. Let us now define: for $t \in[a, b]$,

$$
\tilde{\gamma}(t):=\phi^{-1}\left(\gamma_{T}(t), 0\right) \in \partial \Omega .
$$

For almost every $t \in(a, b)$, if $\gamma(t) \in \partial \Omega, \gamma(t)=\phi^{-1}\left(\gamma_{T}(t), 0\right)=\tilde{\gamma}(t), g(\gamma(t))=g(\tilde{\gamma}(t))$ and

$$
\begin{aligned}
\left|\gamma^{\prime}(t)\right|^{2} & =\left[\left(\gamma_{T}, \gamma_{N}\right)^{\prime}\right]^{T r} G\left(\gamma_{T}(t), 0\right)\left(\gamma_{T}, \gamma_{N}\right)^{\prime} \\
& =\left[\gamma_{T}^{\prime}(t)\right]^{T r} \tilde{G}\left(\gamma_{T}(t), 0\right) \gamma_{T}^{\prime}(t)+G_{N N}\left(\gamma_{T}(t), 0\right) \gamma_{N}^{\prime}(t)^{2} \\
& \geq\left[\gamma_{T}^{\prime}(t)\right]^{T r} \tilde{G}\left(\gamma_{T}(t), 0\right) \gamma_{T}^{\prime}(t)=\left|\tilde{\gamma}^{\prime}(t)\right|^{2},
\end{aligned}
$$

where the supersript ${ }^{T r}$ stands for the transposition operator. For almost every $t \in(a, b)$, if $\gamma(t) \in \Omega$,

$$
\begin{aligned}
\left|\gamma^{\prime}(t)\right|^{2} & =\left[\left(\gamma_{T}, \gamma_{N}\right)^{\prime}\right]^{T r} G\left(\left(\gamma_{T}, \gamma_{N}\right)\right)\left(\gamma_{T}, \gamma_{N}\right)^{\prime} \\
& =\left[\gamma_{T}^{\prime}(t)\right]^{T r} \tilde{G}\left(\left(\gamma_{T}, \gamma_{N}\right)\right) \gamma_{T}^{\prime}(t)+G_{N N}\left(\left(\gamma_{T}, \gamma_{N}\right)\right) \gamma_{N}^{\prime}(t)^{2} \\
& \geq(1-\varepsilon)^{2}\left[\gamma_{T}^{\prime}(t)\right]^{T r} \tilde{G}\left(\gamma_{T}(t), 0\right) \gamma_{T}^{\prime}(t)=(1-\varepsilon)^{2}\left|\tilde{\gamma}^{\prime}(t)\right|^{2} .
\end{aligned}
$$

Step 2: Definition of $\gamma_{12}$. Let $\left(t_{1}, t_{2}\right) \in[0,1]^{2}$, with $a \leq t_{1}<t_{2} \leq b$.

Let us distinguish between two cases. 
- If the set $\left\{t \in\left[t_{1}, t_{2}\right], \gamma(t) \in \partial \Omega\right\}$ is non empty, let us consider $t_{1}^{+}:=$ $\inf \left\{t \in\left[t_{1}, t_{2}\right], \gamma(t) \in \partial \Omega\right\}$ and $t_{2}^{-}:=\sup \left\{t \in\left[t_{1}, t_{2}\right], \gamma(t) \in \partial \Omega\right\}$. The curve $\gamma_{12}:\left[t_{1}, t_{2}\right] \rightarrow \bar{\Omega}$ is then defined by

$$
\gamma_{12}(t)= \begin{cases}\gamma(t) & \text { if } t \in\left(t_{1}, t_{1}^{+}\right) \\ \tilde{\gamma}(t) & \text { if } t \in\left(t_{1}^{+}, t_{2}^{-}\right), \\ \gamma(t) & \text { if } t \in\left(t_{2}^{-}, t_{2}\right)\end{cases}
$$

Observe that for all $t \in\left(t_{1}, t_{1}^{+}\right) \cup\left(t_{2}^{-}, t_{2}\right), \gamma(t)=\gamma_{12}(t)$, which implies $g(\gamma(t))\left|\gamma^{\prime}(t)\right|=g\left(\gamma_{12}(t)\right)\left|\tilde{\gamma}_{12}^{\prime}(t)\right|$ almost everywhere in $\left(t_{1}, t_{1}^{+}\right) \cup\left(t_{2}^{-}, t_{2}\right)$. Moreover, using the fact that $A(1-\varepsilon)>1$, for almost every $t \in\left(t_{1}^{+}, t_{2}^{-}\right)$,

$$
g(\gamma(t))\left|\gamma^{\prime}(t)\right| \geq A(1-\varepsilon) g\left(\phi^{-1}\left(\gamma_{T}(t), 0\right)\right)\left|\tilde{\gamma}^{\prime}(t)\right|>g(\tilde{\gamma})\left|\tilde{\gamma}^{\prime}(t)\right|=g\left(\gamma_{12}\right)\left|\gamma_{12}^{\prime}(t)\right| .
$$

Therefore 72 is satisfied.

- If the set $\left\{t \in\left[t_{1}, t_{2}\right], \gamma(t) \in \partial \Omega\right\}$ is empty, which means that $\forall t \in\left[t_{1}, t_{2}\right], \gamma(t) \in \Omega$, then one simply defines the curve $\gamma_{12}:\left[t_{1}, t_{2}\right] \rightarrow \bar{\Omega}$ by $\gamma_{12}=\gamma$.

In both cases, the curve $\gamma_{12}$ is Lipschitz, $\gamma_{12}\left(t_{j}\right)=\gamma\left(t_{j}\right)$ for $j \in\{1,2\}$ and 72 is satisfied. Moreover by construction of $\gamma_{12}$, the set $\partial\left\{t \in\left[t_{1}, t_{2}\right], \gamma_{12}(t) \in \partial \Omega\right\}$ consists of isolated points in $\left\{t \in\left[t_{1}, t_{2}\right], \gamma_{12}(t) \in \partial \Omega\right\}$, or is empty.

Step 3: On the strict inequality in $(72)$.

Assume that $(73)$ holds and let us show that the inequality $(72)$ is strict. Indeed, in that case $t_{1}^{+} \leq s_{1}<s_{3} \leq t_{2}^{-}$and by continuity of $\gamma$, there exists $\left(u_{1}, u_{2}\right) \in\left(s_{1}, s_{3}\right)^{2}$ such that $u_{1}<s_{2}<u_{2}$ and $\gamma\left(\left[u_{1}, u_{2}\right]\right) \subset \Omega$. Thus, the inequality $(75)$ holds almost everywhere on the open nonempty interval $\left(u_{1}, u_{2}\right)$ which implies that $L\left(\gamma,\left(u_{1}, u_{2}\right)\right)>L\left(\gamma_{12},\left(u_{1}, u_{2}\right)\right)$. This concludes the proof of Proposition 36 .

A consequence of the previous proposition is the following result.

Proposition 38. Let $x, y \in \bar{\Omega}$ and assume that [H3] holds. For any Lipschitz curve $\gamma$ : $[0,1] \rightarrow \bar{\Omega}$ with $\gamma(0)=x$ and $\gamma(0)=y$, there exists $\gamma_{1} \in A(x, y)$ such that $L(\gamma,(0,1)) \geq$ $L\left(\gamma_{1},(0,1)\right)$.

Proof. The set $\partial\{t \in[0,1], \gamma(t) \in \partial \Omega\}$ is closed, so its limit points are its non isolated points. Let us define $\operatorname{Ad}(\gamma)$ as the set of limit points of $\partial\{t \in[0,1], \gamma(t) \in \partial \Omega\}$. If $\operatorname{Ad}(\gamma)$ is empty, then $\partial\{t \in[0,1], \gamma(t) \in \partial \Omega\}$ is empty or consists of isolated points in $\partial\{t \in[0,1], \gamma(t) \in \partial \Omega\}$ and since $\partial\{t \in[0,1], \gamma(t) \in \partial \Omega\}$ is compact, this implies that $\gamma \in A(x, y)$ and Proposition 38 is thus proved by simply taking $\gamma_{1}=\gamma$.

If $\operatorname{Ad}(\gamma)$ is non empty, we will construct a curve $\gamma_{1} \in A(x, y)$ such that

$$
L(\gamma,(0,1)) \geq L\left(\gamma_{1},(0,1)\right)
$$

Without loss of generality, one can assume that 0 and 1 are not in $\operatorname{Ad}(\gamma)$. Otherwise one could modify $\gamma$ in neighborhoods of 0 and 1 without increasing $L(\gamma,(0,1))$ and without changing the end points using Proposition 36. To prove the result, we will 
show by induction on $N \geq 1$ the following property $\mathcal{P}_{N}$ : for all $\left\{t_{1}, \ldots, t_{N}\right\} \subset \operatorname{Ad}(\gamma)$, denote by $\left(a_{j}, b_{j}\right)_{j=1, \ldots, N}$ the open intervals given by Proposition 36 for each $t_{i}$; then, it is possible to change $\gamma$ to construct a Lipschitz curve $\gamma_{1}:[0,1] \rightarrow \bar{\Omega}$ with $\gamma_{1}(0)=x$ and $\gamma_{1}(0)=y$, such that $\gamma_{1}=\gamma$ on $[0,1] \backslash\left(\bigcup_{j=1}^{N}\left(a_{j}, b_{j}\right)\right)^{c}, \operatorname{Ad}\left(\gamma_{1}\right) \cap \bigcup_{j=1}^{N}\left(a_{j}, b_{j}\right)=\emptyset$ and $L(\gamma,(0,1)) \geq L\left(\gamma_{1},(0,1)\right)$.

The first step to prove $\mathcal{P}_{1}$ is just a straightforward application of Proposition 36 (choosing $t_{1}=a_{1}$ and $\left.t_{2}=b_{2}\right)$. Now, let us prove $\mathcal{P}_{N+1}$ assuming $\mathcal{P}_{N}$. Let us consider $\left\{t_{1}, \ldots, t_{N}, t_{N+1}\right\} \subset \operatorname{Ad}(\gamma)$ and denote by $\left(a_{j}, b_{j}\right)_{j=1, \ldots, N, N+1}$ the open intervals given by Proposition 36 for each $t_{i}$. Applying $\mathcal{P}_{N}$, it is possible to change $\gamma$ to construct a Lipschitz curve $\gamma_{1}:[0,1] \rightarrow \bar{\Omega}$ with $\gamma_{1}(0)=x$ and $\gamma_{1}(0)=y$, such that $\operatorname{Ad}\left(\gamma_{1}\right) \cap$ $\bigcup_{j=1}^{N}\left(a_{j}, b_{j}\right)=\emptyset$ and $L(\gamma,(0,1)) \geq L\left(\gamma_{1},(0,1)\right)$. If $\left(a_{N+1}, b_{N+1}\right) \subset \bigcup_{j=1}^{N}\left(a_{j}, b_{j}\right)$, then $\mathcal{P}_{N+1}$ holds taking $\gamma_{1}$. Otherwise, there exist $K \in \mathbb{N}^{*}$ and $\left(q_{1}, \ldots, q_{K}, d_{1}, \ldots, d_{K}\right) \in$ $[0,1]^{2 K}$ such that

$$
\left(a_{N+1}, b_{N+1}\right) \cap\left[\bigcup_{j=1}^{N}\left(a_{j}, b_{j}\right)\right]^{c}=\bigcup_{i=1}^{K}\left[\left(q_{i}, d_{i}\right)\right],
$$

with $0<q_{1}<d_{1}<q_{2}<d_{2}<\ldots<q_{K}<d_{K}<1$; the notation [( and )] mean that the extremities can or not belong to the interval. In addition, for $i \in\{1, \ldots, K\}, q_{i} \in$ $\left\{a_{N+1}\right\} \cup\left\{b_{1}, \ldots, b_{N}\right\}$ and $d_{i} \in\left\{b_{N+1}\right\} \cup\left\{a_{1}, \ldots, a_{N}\right\}$. Then applying Proposition 36 to $\gamma_{1}$ on each interval $\left(q_{i}, d_{i}\right) \subset\left(a_{N+1}, b_{N+1}\right)$, one gets that it is possible to construct a Lipschitz curve $\gamma_{2}$ with $\gamma_{2}(0)=x$ and $\gamma_{2}(0)=y$, such that

$$
L\left(\gamma_{1},(0,1)\right) \geq L\left(\gamma_{2},(0,1)\right), \operatorname{Ad}\left(\gamma_{2}\right) \cap \bigcup_{i=1}^{K}\left(q_{i}, d_{i}\right)=\emptyset .
$$

If for some $k \in\{1, \ldots, K\}$ and $j \in\{1, \ldots, N\} q_{k}=b_{j}$, then $q_{k}$ is isolated from the left in $\left\{t \in[0,1], \gamma_{1}(t) \in \partial \Omega\right\}$ from the construction of $\gamma_{1}$ (see Proposition 36) and, by construction of $\gamma_{2}, q_{k}$ is also isolated from the right in $\left\{t \in[0,1], \gamma_{2}(t) \in \partial \Omega\right\}$. Thus, since there exists $s \in\left[0, q_{k}\right)$ such that $\gamma_{2}=\gamma_{1}$ on $\left[s, q_{k}\right]$, one has $q_{k} \notin \operatorname{Ad}\left(\gamma_{2}\right)$. A similar reasoning holds for the points $d_{k}$. Thus

$$
\operatorname{Ad}\left(\gamma_{2}\right) \cap\left(a_{N+1}, b_{N+1}\right) \cap\left[\bigcup_{j=1}^{N}\left(a_{j}, b_{j}\right)\right]^{c}=\emptyset .
$$

This proves that $\operatorname{Ad}\left(\gamma_{2}\right) \cap \bigcup_{j=1}^{N+1}\left(a_{j}, b_{j}\right)=\emptyset$ and thus $\mathcal{P}_{N+1}$.

By induction, we thus have proven $\mathcal{P}_{N}$ for all $N \geq 1$. Now, notice that by a compactness argument, there exist $N \geq 0$ and $\left\{t_{1}, \ldots, t_{N}\right\} \subset \operatorname{Ad}(\gamma)$ such that if one denotes by $\left(a_{j}, b_{j}\right)_{j=1, \ldots, N}$ the open intervals given by Proposition 36 for each $t_{i}$, then

$$
\operatorname{Ad}(\gamma) \subset \bigcup_{i=1}^{N}\left(a_{i}, b_{i}\right)
$$

Applying $\mathcal{P}_{N}$ yields the desired result.

A direct consequence of Proposition 38 is the following. 
Corollary 39. Assume that [H3] holds. Then the Agmon distance $d_{a}$ introduced in Definition 8 satisfies (70): for all $(x, y) \in \bar{\Omega}^{2}$

$$
d_{a}(x, y)=\inf _{\gamma \in A(x, y)} L(\gamma,(0,1)) .
$$

\subsection{First properties of the Agmon distance}

In this section, we aim at giving the basic properties of the Agmon distance.

\subsubsection{Upper bounds on $d_{a}$ and topology of $\left(\bar{\Omega}, d_{a}\right)$}

Proposition 40. The Agmon distance $(x, y) \in \bar{\Omega} \times \bar{\Omega} \mapsto d_{a}(x, y)$ is symmetric and satisfies the triangular inequality. Moroever, there exists a constant $C$ such that for all $x, y \in \bar{\Omega}$,

$$
d_{a}(x, y) \leq C|x-y|
$$

For any fixed $y \in \bar{\Omega}, x \in \bar{\Omega} \mapsto d_{a}(x, y)$ is Lipschitz. Its gradient is well defined almost everywhere and satisfies for $y \in \bar{\Omega}$ and for almost every $x \in \Omega$,

$$
\left|\nabla_{x} d_{a}(x, y)\right| \leq|\nabla f(x)| .
$$

Moreover, if [H3] holds, for all $x, y \in \bar{\Omega}$, we have

$$
|f(x)-f(y)| \leq d_{a}(x, y) .
$$

Proof. The inequality (76) is proved below in Lemma 41. The proof of $(77)$ is standard, see for instance $[25$, p. 53]. For the ease of the reader, let us recall the proof. Let $y \in \bar{\Omega}$ and $x \in \Omega$. Since $\Omega$ is open, there exists an open ball $B \subset \Omega$ with center $x$. Let $z \in B$. For $t \in[0,1]$, the path $\gamma(t)=t z+(1-t) x$ is included in $B$. Then, one obtains

$$
\begin{aligned}
\left|d_{a}(x, y)-d_{a}(z, y)\right| \leq d_{a}(x, z) & \leq|x-z| \int_{0}^{1} g(t z+(1-t) x) d t \\
& =|x-z| \int_{0}^{1}|\nabla f|(x+t(z-x)) d t .
\end{aligned}
$$

Since $f$ is smooth, up to considering a smaller ball $B$ centered at $x$, there exists a constant $c>0$ such that for all $z \in B$, for all $t \in[0,1],|\nabla f|(x+t(z-x)) \leq|\nabla f|(x)+$ $c|x-z|$. Thus, for all $z \in B$, it holds $\left|d_{a}(x, y)-d_{a}(z, y)\right| \leq|x-z|(|\nabla f|(x)+c|x-z|)$. As a consequence, for any fixed $y \in \bar{\Omega}$ and for almost $x \in \Omega$ one gets $(77)$, by considering the limit $z \rightarrow x$ in the previous inequality. Let us now prove the inequality (78). For any $\gamma \in A(x, y)$, using Lemma 34 , one has:

$$
\begin{aligned}
|f(x)-f(y)| & =\left|\int_{0}^{1} \frac{d}{d t}(f \circ \gamma) d t\right| \\
& =\left|\int_{\{t \in[0,1], \gamma(t) \in \Omega\}}(\nabla f)(\gamma) \cdot \gamma^{\prime} d t+\int_{\operatorname{int}\{t \in[0,1], \gamma(t) \in \partial \Omega\}}\left(\nabla_{T} f\right)(\gamma) \cdot \gamma^{\prime} d t\right| \\
& \leq \int_{0}^{1} g(\gamma(t))\left|\gamma^{\prime}(t)\right| d t .
\end{aligned}
$$

Therefore $(78)$ is proved by taking the infimum over $\gamma \in A(x, y)$ in the right-hand side (see Corollary 39). 
Let us now give the proof of $(76)$.

Lemma 41. The function $(x, y) \in \bar{\Omega} \times \bar{\Omega} \mapsto d_{a}(x, y)$ is bounded and satisfies

$$
\sup _{(x, y) \in \bar{\Omega} \times \bar{\Omega}, x \neq y} \frac{d_{a}(x, y)}{|x-y|}<\infty .
$$

Proof. Let us first prove by contradiction that $(x, y) \in \bar{\Omega} \times \bar{\Omega} \mapsto d_{a}(x, y)$ is bounded. Let us assume that there exists a sequence $\left(x_{k}, y_{k}\right)_{k \geq 1} \in \bar{\Omega} \times \bar{\Omega}$ such that for all $k \geq 1$,

$$
d_{a}\left(x_{k}, y_{k}\right) \geq k
$$

Up to the extraction of a subsequence, it can be assumed that $\lim _{k \rightarrow \infty}\left(x_{k}, y_{k}\right)=(x, y) \in$ $\bar{\Omega} \times \bar{\Omega}$ (the convergence being for the Euclidean metric). Notice that

$$
d_{a}\left(x_{k}, y_{k}\right) \leq d_{a}\left(x_{k}, x\right)+d_{a}(x, y)+d_{a}\left(y_{k}, y\right) .
$$

Let us consider $d_{a}\left(x_{k}, x\right)$. If $x \in \Omega$ then there exist an open ball $B \subset \Omega$ centered on $x$ and an integer $N$ such that for all $k \geq N, x_{k} \in B$ and therefore $\gamma(t)=t x_{k}+(1-t) x \in B$ for all $t \in(0,1)$. Then, by definition of the Agmon distance, for all $k \geq 1$,

$$
d_{a}\left(x_{k}, x\right) \leq\|g\|_{L^{\infty}(B)}\left|x-x_{k}\right| .
$$

If $x \in \partial \Omega$ then there exist $r>0$ and a $C^{\infty}$ bijective map $\phi: B(x, r) \rightarrow B(0,1)$ such that $\phi(x)=0, \phi(B(x, r) \cap \partial \Omega)=Q_{0}$ and $\phi(B(x, r) \cap \bar{\Omega})=Q_{-}$, where $Q_{0}:=\{y=$ $\left.\left(y_{1}, \ldots, y_{d}\right),|y| \leq 1, y_{d}=0\right\}$ and $Q_{-}:=\left\{y=\left(y_{1}, \ldots, y_{d}\right),|y| \leq 1, y_{d} \leq 0\right\}$. Moreover, there exists $N$ such that for all $k \geq N, x_{k} \in B(x, r) \cap \bar{\Omega}$. Now, for any $k \geq N$, let us consider $\gamma(t)=\phi^{-1}\left((1-t) \phi\left(x_{k}\right)+t \phi(x)\right)$. Notice that $\gamma \in A\left(x_{k}, x\right)$ and satisfies $\gamma([0,1]) \subset B(x, r)$. Moreover $c(t)=\phi(\gamma(t))=(1-t) \phi\left(x_{k}\right)+t \phi(x) \in Q_{-}$for $t \in[0,1]$. Then, one has:

$$
\begin{aligned}
d_{a}\left(x_{k}, x\right) \leq \int_{0}^{1} g(\gamma)\left|\gamma^{\prime}\right| & \leq\|g\|_{L^{\infty}(B(x, r))} \int_{0}^{1}\left|\gamma^{\prime}\right| \\
& =\|g\|_{L^{\infty}(B(x, r))} \int_{0}^{1}\left|\operatorname{Jac}\left(\phi^{-1}\right)(c) c^{\prime}\right| \\
& \leq\|g\|_{L^{\infty}(B(x, r))}\left\|\operatorname{Jac}\left(\phi^{-1}\right)\right\|_{L^{\infty}(B(0,1))}\left|\phi\left(x_{k}\right)-\phi(x)\right|,
\end{aligned}
$$

and therefore, since $\phi$ is Lipschitz,

$$
d_{a}\left(x_{k}, x\right) \leq C\left|x_{k}-x\right|,
$$

where $C$ is a constant independent of $k \geq N$. This shows that $d_{a}\left(x_{k}, x\right)$ is bounded. The same reasoning shows that $d_{a}\left(y, y_{k}\right)$ is bounded. This yields a contradiction, considering the inequality (81) and 80 .

To show $(79)$, one proceeds in the same way. Assume that there exists a sequence $\left(x_{k}, y_{k}\right) \in \bar{\Omega} \times \bar{\Omega}$ such that

$$
d_{a}\left(x_{k}, y_{k}\right) \geq k\left|x_{k}-y_{k}\right|
$$

Up to the extraction of a subsequence, it can be assumed that $\lim _{k \rightarrow \infty}\left(x_{k}, y_{k}\right)=(x, y) \in$ $\bar{\Omega} \times \bar{\Omega}$. If $x \neq y$, then, for $k$ sufficiently large

$$
\frac{d_{a}\left(x_{k}, y_{k}\right)}{\left|x_{k}-y_{k}\right|} \leq 2 \frac{\sup _{(x, y) \in \bar{\Omega}} d_{a}(x, y)}{|x-y|}<\infty
$$


which contradicts (83). If $x=y \in \Omega$, then, for all $k$ sufficiently large, the curve $\gamma(t)=t x_{k}+(1-t) y_{k}$ is with values in $\Omega$ and therefore for all $k$ sufficiently large, $d_{a}\left(x_{k}, y_{k}\right) \leq\|g\|_{L^{\infty}(\bar{\Omega})}\left|y_{k}-x_{k}\right|$. This again leads to a contradiction when $k \rightarrow \infty$. Finally, if $x=y \in \partial \Omega$, using the same reasoning as above to prove 82 , one has the existence of a constant $C$ such that for all $k$ sufficiently large, $d_{a}\left(x_{k}, y_{k}\right) \leq C\left|x_{k}-y_{k}\right|$, which again contradicts (83). This concludes the proof of Lemma 41 .

A consequence of the previous lemma is the following proposition.

Proposition 42. Assume that [H1] holds. The space $\left(\bar{\Omega}, d_{a}\right)$ is a compact separated metric space. Moreover the topology of the metric space $\left(\bar{\Omega}, d_{a}\right)$ is equivalent to the topology induced by the Euclidean metric on $\bar{\Omega}$.

Proof. Let us show that for any $(x, y) \in \bar{\Omega} \times \bar{\Omega}$, if $x \neq y$ then $d_{a}(x, y)>0$. Let us denote by $d_{e}$ the geodesic distance on $\bar{\Omega}$ for the Euclidean metric: for all $x, y \in \bar{\Omega}$,

$$
d_{e}(x, y):=\inf _{\gamma} \int_{0}^{1}\left|\gamma^{\prime}(t)\right| d t
$$

where the infimum is taken over all the paths $\gamma:[0,1] \rightarrow \bar{\Omega}$ which are Lipschitz with $\gamma(0)=x$ and $\gamma(1)=y$. Since [H1] holds, the functions $f$ and $\left.f\right|_{\partial \Omega}$ have a finite number of critical points, and thus, there exist $0<r_{1}<r_{2}<d_{e}(x, y)$ such that the infimum of $g$ on the set $C\left(r_{1}, r_{2}\right):=\left\{z \in \bar{\Omega}, r_{1}<d_{e}(x, z)<r_{2}\right\}$ is positive i.e. $c\left(r_{1}, r_{2}\right):=\inf _{C\left(r_{1}, r_{2}\right)} g>0$. For any path $\gamma \in A(x, y)$, one has

$$
\int_{0}^{1}\left|\gamma^{\prime}(t)\right| g(\gamma(t)) d t \geq c\left(r_{1}, r_{2}\right) r\left(C\left(r_{1}, r_{2}\right)\right)
$$

where $r\left(C\left(r_{1}, r_{2}\right)\right):=\inf _{z \in C\left(r_{1}, r_{2}\right)} \sup _{y \in C\left(r_{1}, r_{2}\right)} d_{e}(z, y)>0$. Then $d_{a}(x, y)>0$. If $x=y$, it is clear that $d_{a}(x, y)=0$ since $L(\gamma,(0,1))=0$ where $\gamma(t)=x$ for all $t \in[0,1]$. This shows that $\left(\bar{\Omega}, d_{a}\right)$ is separated.

The fact that $\left(\bar{\Omega}, d_{a}\right)$ is compact comes from the inequality 76 proved in Lemma 41 . Indeed, since $\left(\bar{\Omega}, d_{a}\right)$ is a metric space, it is sufficient to prove the sequential compactness. Let $\left(x_{n}\right)_{n \geq 0}$ be a sequence in $\bar{\Omega}$. Since $\bar{\Omega}$ is compact for the Euclidean metric, one can extract a converging subsequence $\left(x_{n}^{\prime}\right)_{n \geq 0}$ for the Euclidean metric. From $(76)$, this subsequence is also converging for $d_{a}$, which ends the proof.

Let us finally prove the equivalence of the topologies on $\bar{\Omega}$. From Lemma 41, it is obvious that if a sequence $\left(x_{n}\right)_{n \geq 0}$ converges to $x$ in $\bar{\Omega}$ for the Euclidean metric, then $d_{a}\left(x_{n}, x\right)$ converges to 0 . Conversely, let us assume that $\left(x_{n}\right)_{n \geq 0}$ is a sequence of $\bar{\Omega}$ such that $d_{a}\left(x_{n}, x\right)$ converges to 0 , for a point $x \in \bar{\Omega}$. Since $\bar{\Omega}$ is compact for the Euclidean metric, it is enough to show that $x$ is the only limit point of the sequence to show that $\left(x_{n}\right)_{n \geq 0}$ converges to $x$ for the Euclidean metric. From Lemma 41 , any limit point $y$ for the Euclidean metric is also an limit point for the Agmon distance, and thus, since $\left(\bar{\Omega}, d_{a}\right)$ is a separated space, $y=x$. This concludes the proof.

Notice that from the proof, it is obvious that the topology is separated as soon as $f$ and $\left.f\right|_{\partial \Omega}$ have a finite number of critical points, which is a weaker assumption than $[\mathbf{H 1}]$.

Finally, the following lemma will be useful in the following. 
Lemma 43. Assume that [H3] holds. Let $I \subset \mathbb{R}$ be an interval and $\gamma: I \rightarrow \bar{\Omega}$ a Lipschitz curve such that $\partial\{t \in I, \gamma(t) \in \partial \Omega\}$ is finite and such that $x:=\lim _{t \rightarrow(\inf I)^{+}} \gamma(t)$ and $y:=\lim _{t \rightarrow(\sup I)^{-}} \gamma(t)$ exist. Then one has

$$
d_{a}(x, y) \leq L(\gamma, I) .
$$

Proof. Let $(a, b) \in I^{2}$ with $a<b$ and define for $u \in[0,1], \gamma_{a b}(u)=\gamma(a+u(b-a))$. Then $\gamma_{a b} \in A(\gamma(a), \gamma(b))$. By definition of the Agmon distance (see Definition 8), $d_{a}(a, b) \leq L\left(\gamma_{a b},(0,1)\right)=L(\gamma,(a, b))$. Taking the limits $a \rightarrow(\inf I)^{+}, b \rightarrow(\sup I)^{-}$ and using the continuity of the Agmon distance, one obtains that $d_{a}(x, y) \leq L(\gamma, I)$. Lemma 43 is proved.

As a simple consequence of this lemma, we have the following simple remark.

Remark 44. Let $x^{*}$ be a local minimum of $f$ (from [H3], $x^{*} \in \Omega$ ). Then, for all $x$ in the basin of attraction of $x^{*}$ for the dynamics

$$
\gamma^{\prime}=\left\{\begin{array}{c}
-\nabla f(\gamma) \text { in } \Omega \\
-\nabla_{T} f(\gamma) \text { on } \partial \Omega
\end{array}\right.
$$

it holds $x \in \Omega$ and

$$
d_{a}\left(x^{*}, x\right)=f(x)-f\left(x^{*}\right) .
$$

Indeed, from (78), we already have $d_{a}\left(x^{*}, x\right) \geq f(x)-f\left(x^{*}\right)$. To prove the reverse inequality, from Lemma 43, it is enough to exhibit a Lipschitz curve $\tilde{\gamma}: I \rightarrow \bar{\Omega}$ such that $\partial\{t \in I, \tilde{\gamma}(t) \in \partial \Omega\}$ is finite, $L(\tilde{\gamma}, I)=f(x)-f\left(x^{*}\right)$ and $\lim _{t \rightarrow(\inf I)^{+}} \tilde{\gamma}(t)=x^{*}$ and $\lim _{t \rightarrow(\sup I)^{-}} \tilde{\gamma}(t)=x$. Such a curve is given on the interval $I=(-\infty, 0]$ by $\tilde{\gamma}: t \in$ $I \mapsto \gamma(-t)$ where $\gamma$ is the solution to 85 with initial condition $\gamma(0)=x$. Notice that if $\exists t_{0}$ such that $\tilde{\gamma}\left(t_{0}\right) \in \partial \Omega$, then $\forall t \leq t_{0}, \tilde{\gamma}(t) \in \partial \Omega$. Thus, since $\lim _{t \rightarrow-\infty} \tilde{\gamma}(t)=x^{*} \in \Omega$, one has: for all $t \leq 0, \tilde{\gamma}(t) \in \Omega$. Therefore,

$$
\begin{aligned}
f(x)-f\left(x^{*}\right) & =\int_{-\infty}^{0} \frac{d}{d t} f \circ \tilde{\gamma}(t) d t=\int_{-\infty}^{0} \nabla f(\tilde{\gamma}(t)) \cdot \tilde{\gamma}^{\prime}(t) d t=\int_{-\infty}^{0}|\nabla f(\tilde{\gamma}(t))|^{2} d t \\
& =\int_{-\infty}^{0}|\nabla f(\tilde{\gamma}(t))|\left|\tilde{\gamma}^{\prime}(t)\right| d t=\int_{-\infty}^{0} g(\tilde{\gamma}(t))\left|\tilde{\gamma}^{\prime}(t)\right| d t \\
& =\lim _{t \rightarrow-\infty} L(\tilde{\gamma},(t, 0)) .
\end{aligned}
$$

This concludes the proof of 86 . Notice that for $\varepsilon>0$ small enough, the set $f^{-1}\left(\left[f\left(x^{*}\right), f\left(x^{*}\right)+\varepsilon\right)\right) \cap$ $B\left(x^{*}, \varepsilon\right) \subset \Omega$ is a neighborhood of $x^{*}$ which is included in the basin of attraction of $x^{*}$ for the dynamics (85). Therefore (86) holds in a neighborhood of $x^{*}$.

\subsubsection{A lower bound on the Agmon distance}

In this section, easily computable lower bounds on the Agmon distance are provided. This is in particular useful to check if the hypothesis 20 appearing in Theorem 1 is satisfied, see for example Section 1.6.2.

Proposition 45. Let $z \in \bar{\Omega}$ and denote by $W$ and $W^{\prime}$ two closed neighborhoods of $z$ in $\bar{\Omega}$ with $W \subset W^{\prime}$. Define

$$
\alpha:=\inf \left\{d_{e}(x, y), x \in \bar{\Omega} \backslash W^{\prime}, y \in W\right\},
$$


where $d_{e}$ denotes the geodesic distance for the Euclidean metric, see 84. Assume that $\alpha>0$ and that there exists $K>0$ such that

$$
\inf _{x \in W^{\prime} \backslash W} g(x)>\frac{K}{\alpha},
$$

where $g$ has been introduced in Definition 7 . Then, for all set $B \subset \bar{\Omega}$ such that $B \cap \overline{W^{\prime}}=$ $\emptyset$,

$$
\inf _{y \in B} d_{a}(z, y)>K
$$

where $d_{a}$ is the Agmon distance (see Definition 8).

Proof. By assumption, there exists $\varepsilon>0$ such that

$$
\inf _{x \in \overline{W^{\prime} \backslash W}} g(x) \geq \frac{K}{\alpha}+\varepsilon .
$$

Let $y \in B$ and $\gamma \in \operatorname{Lip}(z, y)$. Let us define

$$
t_{2}=\inf \left\{t \in[0,1], \gamma(t) \notin W^{\prime}\right\}, t_{1}=\sup \left\{t \in[0,1], t<t_{2}, \gamma(t) \in W\right\} .
$$

Since $\gamma$ is continuous and $\alpha>0$, it holds $0<t_{1}<t_{2}<1$ and one has $\gamma(t) \in \overline{W^{\prime} \backslash W}$ for all $t \in\left[t_{1}, t_{2}\right], \gamma\left(t_{1}\right) \in \bar{W}=W$ and $\gamma\left(t_{2}\right) \in \overline{\bar{\Omega} \backslash W^{\prime}}$. Then, one has:

$$
\begin{aligned}
\int_{0}^{1} g(\gamma(t))\left|\gamma^{\prime}(t)\right| d t & \geq \int_{t_{1}}^{t_{2}} g(\gamma(t))\left|\gamma^{\prime}(t)\right| d t \\
& \geq\left(\frac{K}{\alpha}+\varepsilon\right) \int_{t_{1}}^{t_{2}}\left|\gamma^{\prime}(t)\right| d t \\
& \geq\left(\frac{K}{\alpha}+\varepsilon\right) \alpha=K+\varepsilon \alpha .
\end{aligned}
$$

Since $\varepsilon \alpha$ is independent of $y \in B$ and since $\varepsilon \alpha$ is also independent of the curve $\gamma$, one can take the infimum over $\gamma$ and $y \in B$. Thus $\inf _{y \in B} d_{a}(z, y)>K$.

We now give a simple sufficient condition for the hypotheses 20 to hold, in the case where $\left.f\right|_{\partial \Omega}$ has only two local minima. This result is based on Proposition 66 that will be proven in Section 3.4 .3 below and which shows that

$$
d_{a}\left(z_{1}, z_{2}\right)>f\left(z_{2}\right)-f\left(z_{1}\right)
$$

In particular, the condition stated in the following proposition has been used in Section 1.6 .2 in order to check hypothesis 200 .

Proposition 46. Assume that [H1] and [H3] hold and assume in addition that $\left.f\right|_{\partial \Omega}$ has only two local minima $z_{1}$ and $z_{2}$ (with $f\left(z_{1}\right) \leq f\left(z_{2}\right)$ ) on $\partial \Omega$. Then, if $z_{2}$ is the only global minimum of $\left.f\right|_{\partial \Omega}$ on $B_{z_{1}}^{c}$, one has

$$
\inf _{z \in B_{z_{1}}^{c}} d_{a}\left(z_{1}, z\right)>f\left(z_{2}\right)-f\left(z_{1}\right)
$$


Proof. Proposition 66 and the continuity of the Agmon distance ensure that there exist an open ball $B_{2} \subset B_{z_{1}}^{c}$ centered at $z_{2}$, and $\varepsilon>0$ such that for all $z \in B_{2}$

$$
d_{a}\left(z_{1}, z\right) \geq f\left(z_{2}\right)-f\left(z_{1}\right)+\varepsilon .
$$

Since $z_{2}$ is the only global minimum of $\left.f\right|_{\partial \Omega}$ on $B_{z_{1}}^{c}$, there exists $\varepsilon^{\prime}>0$, such that for all $z \in B_{z_{1}}^{c} \backslash B_{2}, f(z) \geq f\left(z_{2}\right)+\varepsilon^{\prime}$. In addition, from the inequality (78), for all $z \in B_{z_{1}}^{c} \backslash B_{2}$, it holds

$$
d_{a}\left(z_{1}, z\right) \geq f(z)-f\left(z_{1}\right) \geq f\left(z_{2}\right)-f\left(z_{1}\right)+\varepsilon^{\prime} .
$$

Consequently $\inf _{z \in B_{z_{1}}^{c}} d_{a}\left(z_{1}, z\right)>f\left(z_{2}\right)-f\left(z_{1}\right)$.

\subsection{Agmon distance near critical points of $f$ or $\left.f\right|_{\partial \Omega}$ and eikonal equa- tion}

We will show that the Agmon distance $d_{a}$ locally solves the eikonal equation in a neighborhood of any critical point of $\left.f\right|_{\partial \Omega}$ or $f$ (or equivalently, any point $x$ such that $g(x)=0$, see (17)).

\subsubsection{The Agmon distance near critical points of $f$}

Proposition 47. Let us assume that [H1] holds. Let $x^{*} \in \Omega$ be such that $\nabla f\left(x^{*}\right)=0$. Let us denote by $\left(\mu_{1}, \ldots, \mu_{d}\right) \in\left(\mathbb{R}^{*}\right)^{d}$ the eigenvalues of the Hessian of $f$ at $x^{*}$. Then there exist a neighborhood $V^{*}$ of $x^{*}$ in $\Omega$ and a $C^{\infty}$ function $\Phi: V^{*} \rightarrow \mathbb{R}$ such that

$$
\left\{\begin{aligned}
|\nabla \Phi|^{2} & =|\nabla f|^{2}, \\
\Phi\left(x_{1}, \ldots, x_{d}\right) & =\frac{1}{2} \sum_{i=1}^{d}\left|\mu_{i}\right|\left(x_{i}-x_{i}^{*}\right)^{2}+O\left(\left|x-x^{*}\right|^{3}\right) .
\end{aligned}\right.
$$

Moreover, one has the following uniqueness result: if $\widetilde{\Phi}$ is a $C^{\infty}$ real valued function defined on a neighborhood $\widetilde{V^{*}}$ of $x^{*}$ satisfying $(88)$, then $\widetilde{\Phi}=\Phi$ on $V^{*} \cap \widetilde{V^{*}}$.

Let us notice that $\Phi\left(x^{*}\right)=0$. In addition, up to choosing a smaller neighborhood $V^{*}$ of $x^{*}$, one can assume that $\Phi$ is positive on $V^{*} \backslash\left\{x^{*}\right\}$. The point $x^{*}$ is then a non degenerate minimum of $\Phi$.

Proof. The proof is made in [36, Proposition 2.3.6] in the more general setting where $|\nabla f|^{2}$ is replaced in 88 by a smooth positive function $W$ around a non degenerate minimum $y^{*}$ of $W$ such that $W\left(y^{*}\right)=0$. Here $W=|\nabla f|^{2}$ and $y^{*}=x^{*}$. This leads to $\nabla W=2 \operatorname{Hess} f(\nabla f)$ and thus Hess $W\left(x^{*}\right)=2(\operatorname{Hess} f)^{2}\left(x^{*}\right)$ is a non degenerate matrix. Therefore $x^{*}$ is indeed a non degenerate minimum of $W=|\nabla f|^{2}$.

Proposition 48. Let us assume that [H1] and [H3] hold. Let $x^{*} \in \Omega$ be such that $\nabla f\left(x^{*}\right)=0$. Then there exists a neighborhood $U^{*}$ of $x^{*}$ in $\Omega$ such that for all $x \in U^{*}$

$$
d_{a}\left(x^{*}, x\right)=\Phi(x),
$$

where $\Phi$ is the smooth solution to (88) and $d_{a}$ is the Agmon distance. 
For the ease of the reader, let us give the proof of Proposition 48 which is similar to the proof of [25, Proposition A.1].

Proof. Notice that hypothesis [H3] allows us to use Corollary 39, Let $\Phi$ be a smooth solution to (88) on a neighborhood $V^{*}$ of $x^{*}$, as defined in Proposition 47 and such that $\Phi$ is positive on $V^{*} \backslash\left\{x^{*}\right\}$. There exists $\varepsilon>0$ such that $U^{*}:=\Phi^{-1}([0, \varepsilon)) \subset V^{*}$ is a neighborhood of $x^{*}$ (consider for example $\varepsilon=\inf \left\{\Phi(x), x \in V^{*} \backslash B\left(x^{*}, r\right)\right\}>0$ where $r>0$ is such that $\left.B\left(x^{*}, 2 r\right) \subset V^{*}\right)$.

Let us first prove that for $x \in U^{*}, \Phi(x) \leq d_{a}\left(x, x^{*}\right)$. For $x \in U^{*}$, one has $\Phi(x)<\varepsilon$ and thus $\Phi^{-1}([0, \Phi(x))) \subset U^{*}$. Let $\gamma$ belong to $A\left(x^{*}, x\right)$. Let us define the time $t_{0}:=\inf \left\{t \in[0,1], \gamma(t) \notin \Phi^{-1}([0, \Phi(x)))\right\}$. By continuity of the curve $\gamma$, one has $t_{0}>0$, $\Phi\left(\gamma\left(t_{0}\right)\right)=\Phi(x)$ and for all $t \in\left[0, t_{0}\right), \gamma(t) \in \Phi^{-1}([0, \Phi(x))) \subset U^{*}$. Thus, since the curve $\gamma$ is Lipschitz and since for all $t \in\left[0, t_{0}\right), \gamma(t) \in \Omega$, one has

$$
\begin{aligned}
\Phi(x) & =\int_{0}^{t_{0}} \frac{d}{d t} \Phi(\gamma)(t) d t=\int_{0}^{t_{0}} \nabla \Phi(\gamma(t)) \cdot \gamma^{\prime}(t) d t \\
& \leq \int_{0}^{t_{0}}|\nabla \Phi(\gamma(t))|\left|\gamma^{\prime}(t)\right| d t \\
& \leq \int_{0}^{t_{0}}|\nabla f(\gamma(t))|\left|\gamma^{\prime}(t)\right| d t \leq \int_{0}^{1} g(\gamma(t))\left|\gamma^{\prime}(t)\right| d t=L(\gamma,(0,1)) .
\end{aligned}
$$

Taking the infimum on the right-hand side over $\gamma \in A\left(x^{*}, x\right)$, one gets $\Phi(x) \leq d_{a}\left(x^{*}, x\right)$, for all $x \in U^{*}$. Let us now prove the reverse inequality: for $x \in U^{*}, \Phi(x) \geq d_{a}\left(x, x^{*}\right)$. For $x \in U^{*}$, let us define a curve $\gamma: \mathbb{R}_{+} \rightarrow U^{*}$ by

$$
\forall t \geq 0, \gamma^{\prime}(t)=-\nabla \Phi(\gamma(t)) \text { and } \gamma(0)=x .
$$

Since the function $t \mapsto \Phi(\gamma(t))$ is decreasing, the curve $\gamma$ always belongs to $U^{*}$ and is defined on $\mathbb{R}_{+}$. Moreover $\gamma$ is $C^{\infty}$ and satisfies $\lim _{t \rightarrow+\infty} \gamma(t)=x^{*}$. Since $\gamma$ is with values in $U^{*} \subset \Omega$, one has

$$
\begin{aligned}
-\Phi(x) & =\int_{0}^{+\infty} \frac{d}{d t} \Phi \circ \gamma(t) d t=\int_{0}^{+\infty} \nabla \Phi(\gamma(t)) \cdot \gamma^{\prime}(t) d t=-\int_{0}^{+\infty}|\nabla \Phi(\gamma(t))|^{2} d t \\
& =-\int_{0}^{+\infty}|\nabla \Phi(\gamma(t))|\left|\gamma^{\prime}(t)\right| d t=-\int_{0}^{+\infty} g(\gamma(t))\left|\gamma^{\prime}(t)\right| d t=-\lim _{t \rightarrow+\infty} L(\gamma,(0, t)) .
\end{aligned}
$$

Then, thanks to Lemma 43, it holds: $d_{a}\left(x, x^{*}\right) \leq L(\gamma,(0,+\infty))=\Phi(x)$. Therefore $\Phi(x)=d_{a}\left(x^{*}, x\right)$ for all $x \in U^{*}$.

Remark 49. Let us mention a simple consequence of the previous proof that will be useful in the following. If $x^{*} \in \Omega$ is such that $\nabla f\left(x^{*}\right)=0$, there exists a neighborhood $U^{*}$ of $x^{*}$ in $\Omega$ such that for all $x \in U^{*}$, there exists a $C^{\infty}$ curve $\gamma: \mathbb{R}_{+} \rightarrow \Omega$ such that

$$
d_{a}\left(x^{*}, x\right)=\int_{0}^{+\infty}|\nabla f(\gamma(t))|\left|\gamma^{\prime}(t)\right| d t
$$

with $\gamma(0)=x$ and $\lim _{t \rightarrow+\infty} \gamma(t)=x^{*}$. The curve $\gamma$ is defined by

$$
\gamma^{\prime}(t)=-\nabla \Phi(\gamma(t)), \quad \gamma(0)=x,
$$

where $\Phi$ solves (88). In addition $\{t \in[0, \infty), \gamma(t) \in \partial \Omega\}$ is empty. 


\subsubsection{The Agmon distance near critical points of $\left.f\right|_{\partial \Omega}$}

Let us first define the Agmon distance in the boundary $\partial \Omega$.

Definition 50. The Agmon distance between $x \in \partial \Omega$ and $y \in \partial \Omega$ in the boundary $\partial \Omega$ is defined by

$$
d_{a}^{\partial \Omega}(x, y)=\inf _{\gamma} \int_{0}^{1}\left|\nabla_{T} f(\gamma(t))\right|\left|\gamma^{\prime}(t)\right| d t,
$$

where the infimum is taken over all the paths $\gamma:[0,1] \rightarrow \partial \Omega$ which are Lipschitz with $\gamma(0)=x$ and $\gamma(1)=y$.

Similarly to Remark 44, one has:

Remark 51. If $x^{*}$ is a local minimum of $\left.f\right|_{\partial \Omega}$, one has $d_{a}^{\partial \Omega}\left(x^{*}, x\right)=f(x)-f\left(x^{*}\right)$ for all $x \in \partial \Omega$ which is in the basin of attraction of $x^{*}$ in $\partial \Omega$ for the gradient dynamics $\gamma^{\prime}=-\nabla_{T} f(\gamma)$.

The next proposition is the equivalent of Proposition 48 for that Agmon distance in $\partial \Omega$. Since $\partial \Omega$ is a smooth manifold without boundary, the next result is a direct consequence of well known results from [35, 25] and 28].

Proposition 52. Let us assume that [H1] holds. Let $x^{*} \in \partial \Omega$ be such that $\nabla_{T} f\left(x^{*}\right)=$ 0 . Then there exists a neighborhood $U^{*}$ of $x^{*}$ in $\partial \Omega$ such that $y \mapsto d_{a}^{\partial \Omega}\left(x^{*}, y\right)$ is smooth on $U^{*}$ and $\forall x \in U^{*}$,

$$
\left|\nabla_{T} d_{a}^{\partial \Omega}\left(x^{*}, x\right)\right|^{2}=\left|\nabla_{T} f(x)\right|^{2} .
$$

Proof. The boundary $\partial \Omega$ is a $C^{\infty}$ compact manifold and $x^{*}$ is a non degenerate minimum of $\left|\nabla_{T} f\right|^{2}$. The proof is made in [36, Proposition 2.3.6] in the more general setting where $\left|\nabla_{T} f\right|^{2}$ is replaced in $(92)$ and in $(91)$ by a smooth non negative function $W$ around a non degenerate minimum $y^{*}$ of $W$ such that $W\left(y^{*}\right)=0$. Here $W=\left|\nabla_{T} f\right|^{2}$ and $y^{*}=x^{*}$. This leads to $\nabla W=2 \operatorname{Hess}\left(\left.f\right|_{\partial \Omega}\right)\left(\nabla_{T} f\right)$ and therefore $x^{*}$ is a critical point of $W=\left|\nabla_{T} f\right|^{2}$ (which turns out to be a minimum). In addition, since $\nabla_{T} f\left(x^{*}\right)=0$, one gets that Hess $W\left(x^{*}\right)=2\left(\operatorname{Hess}\left(\left.f\right|_{\partial \Omega}\right)\right)^{2}\left(x^{*}\right)$ which is a non degenerate matrix.

Proposition 53. Let us assume that [H1] and [H3] hold. Let $x^{*} \in \partial \Omega$ be such that $\nabla_{T} f\left(x^{*}\right)=0$. Then, there exist a neighborhood $V^{*}$ of $x^{*}$ in $\bar{\Omega}$ and a $C^{\infty}$ function $\Phi: V^{*} \rightarrow \mathbb{R}$ such that

$$
\left\{\begin{aligned}
|\nabla \Phi|^{2} & =|\nabla f|^{2} \text { in } \Omega \cap V^{*}, \\
\Phi & =d_{a}^{\partial \Omega}\left(x^{*}, .\right) \text { on } \partial \Omega \cap V^{*}, \\
\partial_{n} \Phi & <0 \text { on } \partial \Omega \cap V^{*} .
\end{aligned}\right.
$$

Moreover, one has the following uniqueness results: if $\widetilde{\Phi}$ is a $C^{\infty}$ real valued function defined on a neighborhood $\widetilde{V^{*}}$ of $x^{*}$ satisfying $(93)$, then $\widetilde{\Phi}=\Phi$ on $\widetilde{V^{*}} \cap V^{*}$. Finally, up to choosing a smaller neighborhood $V^{*}$ of $x^{*}$, one can assume that:

- The function $\Phi$ is positive on $V^{*} \backslash\left\{x^{*}\right\}$, so that $x^{*}$ is a non degenerate minimum of $\Phi$ on $V^{*}$.

- According to (92) and (93), it holds on $V^{*} \cap \partial \Omega,\left|\nabla_{T} \Phi\right|=\left|\nabla_{T} f\right|$. 
Proof. From Proposition 52 , the function $x \in \partial \Omega \mapsto d_{a}^{\partial \Omega}\left(x^{*}, x\right)$ is smooth near $x^{*}$. Then, the result stated can be proven using the method of characteristics, see [25, Theorem $1.5]$ or $[28$, Section 3.2]. Let us mention that the proof crucially relies on the assumption $\partial_{n} f\left(x^{*}\right)>0$. The fact that one can reduce $V^{*}$ such that $\Phi$ is positive on $V^{*} \backslash\left\{x^{*}\right\}$ is a consequence of $\partial_{n} \Phi<0$ on $\partial \Omega \cap V^{*}$ together with the fact that $x^{*}$ is the only minimum of $d_{a}^{\partial \Omega}\left(x^{*},.\right)$ (which is positive on $\left.\partial \Omega \backslash\left\{x^{*}\right\}\right)$.

Let us state a simple corollary of Proposition 53 and Remark 51 .

Corollary 54. Let us assume that [H1] and [H3] hold. Let $x^{*} \in \partial \Omega$ be a local minimum of $\left.f\right|_{\partial \Omega}$. Then there exist a neighborhood $V^{*}$ of $x^{*}$ in $\bar{\Omega}$ and a $C^{\infty}$ function $\Phi: V^{*} \rightarrow \mathbb{R}$ such that

$$
\left\{\begin{aligned}
|\nabla \Phi|^{2} & =|\nabla f|^{2} \text { in } \Omega \cap V^{*}, \\
\Phi & =f-f\left(x^{*}\right) \text { on } \partial \Omega \cap V^{*}, \\
\partial_{n} \Phi & <0 \text { on } \partial \Omega \cap V^{*} .
\end{aligned}\right.
$$

Moreover, one has the following uniqueness results: if $\widetilde{\Phi}$ is a $C^{\infty}$ real valued function defined on a neighborhood $\widetilde{V^{*}}$ of $x^{*}$ satisfying $(93)$, then $\widetilde{\Phi}=\Phi$ on $\widetilde{V^{*}} \cap V^{*}$. Finally, up to choosing a smaller neighborhood $V^{*}$ of $x^{*}$, one can assume that $\Phi$ is positive on $V^{*} \backslash\left\{x^{*}\right\}$, and that $\Phi-f>-f\left(x^{*}\right)$ in $V^{*} \cap(\partial \Omega)^{c}$. As a consequence,

$$
\left\{x \in V^{*}, \Phi(x)=f(x)-f\left(x^{*}\right)\right\} \subset \partial \Omega .
$$

Proof. All the statements but (95) are direct consequences of Proposition 53 and the fact that $d_{a}^{\partial \Omega}\left(x^{*}, x\right)=f(x)-f\left(x^{*}\right)$, thanks to Remark 51. Now, notice that on $\partial \Omega \cap V^{*}$, $\Phi-f=-f\left(x^{*}\right)$ and $\partial_{n}(\Phi-f)<0$ so that, up to choosing a smaller neighborhood $V^{*}$ of $x^{*}$, one can assume that $\Phi-f>-f\left(x^{*}\right)$ in $V^{*} \cap(\partial \Omega)^{c}$. This concludes the proof of $(95)$.

We are now in position to state the main result of this section.

Proposition 55. Let us assume that [H1] and [H3] hold. Let $x^{*} \in \partial \Omega$ be such that $\nabla_{T} f\left(x^{*}\right)=0$. Then, there exists a neighborhood $U^{*}$ of $x^{*}$ in $\bar{\Omega}$ such that for all $x \in U^{*}$

$$
d_{a}\left(x, x^{*}\right)=\Phi(x)
$$

where $\Phi$ solves (93) and $d_{a}$ is the Agmon distance.

Proof. Notice that hypothesis [H3] allows us to use Corollary 39. The proof follows the same lines of the proof of Proposition 48 . Let $x^{*} \in \partial \Omega$ be such that $\nabla_{T} f\left(x^{*}\right)=0$. Let $\Phi$ be the smooth solution to (93) on a neighborhood $V^{*}$ of $x^{*}$ and such that $\Phi$ is positive on $V^{*} \backslash\left\{x^{*}\right\}$ and it holds $\left|\nabla_{T} \Phi\right|=\left|\nabla_{T} f\right|$ on $V^{*} \cap \partial \Omega$, as defined in Proposition 53 . One chooses $\varepsilon>0$ sufficiently small such that $U^{*}:=\Phi^{-1}([0, \varepsilon)) \subset V^{*}$. Notice that $U^{*}$ is a neighborhood of $x^{*}$ in $\bar{\Omega}$.

Step 1. Let us first prove that for all $x \in U^{*}, \Phi(x) \leq d_{a}\left(x^{*}, x\right)$.

For $x \in U^{*}$, one has $\Phi(x)<\varepsilon$ and thus $\Phi^{-1}([0, \Phi(x))) \subset U^{*}$. Let $\gamma$ belong to $A\left(x^{*}, x\right)$. Let us define the time $t_{0}:=\inf \left\{t \in[0,1], \gamma(t) \notin \Phi^{-1}([0, \Phi(x)))\right\}$. By continuity of the 
curve $\gamma$, one has $t_{0}>0, \Phi\left(\gamma\left(t_{0}\right)\right)=\Phi(x)$ and for all $t \in\left[0, t_{0}\right), \gamma(t) \in \Phi^{-1}([0, \Phi(x))) \subset$ $U^{*}$. Thus, using Lemma 34 , one obtains

$$
\begin{aligned}
\Phi(x) & =\int_{0}^{t_{0}} \frac{d}{d t} \Phi \circ \gamma(t) d t=\int_{0}^{t_{0}} \nabla \Phi(\gamma(t)) \cdot \gamma^{\prime}(t) d t \\
& =\int_{\operatorname{int}\left\{t \in\left(0, t_{0}\right), \gamma(t) \in \partial \Omega\right\}} \nabla_{T} \Phi(\gamma(t)) \cdot \gamma^{\prime}(t) d t+\int_{\left\{t \in\left(0, t_{0}\right), \gamma(t) \in \Omega\right\}} \nabla \Phi(\gamma(t)) \cdot \gamma^{\prime}(t) d t .
\end{aligned}
$$

On the one hand,

$$
\begin{aligned}
\int_{\operatorname{int}\left\{t \in\left(0, t_{0}\right), \gamma(t) \in \partial \Omega\right\}} \nabla_{T} \Phi(\gamma(t)) \cdot \gamma^{\prime}(t) d t & \leq \int_{\operatorname{int}\left\{t \in\left(0, t_{0}\right), \gamma(t) \in \partial \Omega\right\}}\left|\nabla_{T} \Phi(\gamma(t))\right|\left|\gamma^{\prime}(t)\right| d t \\
& \leq \int_{\operatorname{int}\left\{t \in\left(0, t_{0}\right), \gamma(t) \in \partial \Omega\right\}}\left|\nabla_{T} f(\gamma(t))\right|\left|\gamma^{\prime}(t)\right| d t
\end{aligned}
$$

where one used the last statement in Proposition 53. On the other hand, using (93), one obtains

$$
\begin{aligned}
\int_{\left\{t \in\left(0, t_{0}\right), \gamma(t) \in \Omega\right\}} \nabla \Phi(\gamma(t)) \cdot \gamma^{\prime}(t) d t & \leq \int_{\operatorname{int}\left\{t \in\left(0, t_{0}\right), \gamma(t) \in \Omega\right\}}|\nabla \Phi(\gamma(t))|\left|\gamma^{\prime}(t)\right| d t \\
& \leq \int_{\operatorname{int}\left\{t \in\left(0, t_{0}\right), \gamma(t) \in \Omega\right\}}|\nabla f(\gamma(t))|\left|\gamma^{\prime}(t)\right| d t
\end{aligned}
$$

Thus one gets

$$
\begin{aligned}
\Phi(x) & \leq \int_{\operatorname{int}\left\{t \in\left(0, t_{0}\right), \gamma(t) \in \partial \Omega\right\}}\left|\nabla_{T} f(\gamma(t))\right|\left|\gamma^{\prime}(t)\right| d t+\int_{\left\{t \in\left(0, t_{0}\right), \gamma(t) \in \Omega\right\}}|\nabla f(\gamma(t))|\left|\gamma^{\prime}(t)\right| d t \\
& =\int_{0}^{t_{0}} g(\gamma(t))\left|\gamma^{\prime}(t)\right| d t \leq \int_{0}^{1} g(\gamma(t))\left|\gamma^{\prime}(t)\right| d t=L(\gamma,(0,1)) .
\end{aligned}
$$

Taking the infimum on the right-hand side over $\gamma \in A\left(x^{*}, x\right)$, one gets $\Phi(x) \leq d_{a}\left(x^{*}, x\right)$, for all $x \in U^{*}$.

Step 2. Let us now prove the reverse inequality: $\forall x \in U^{*}, d_{a}\left(x, x^{*}\right) \leq \Phi(x)$.

Let us define the following vector field on $U^{*}$,

$$
X:=\left\{\begin{array}{c}
-\nabla \Phi \text { in } \Omega \cap U^{*}, \\
-\nabla_{T} \Phi \text { on } \partial \Omega \cap U^{*} .
\end{array}\right.
$$

For $x \in U^{*}$, let us define the curve $\gamma$ by

$$
\forall t \geq 0, \gamma^{\prime}(t)=X(\gamma(t)) \text { and } \gamma(0)=x .
$$

This curve is well defined for all positive time using the Cauchy-Lipschitz theorem and the fact that $(\gamma(t))_{t \geq 0}$ remains in $U^{*}$ for all positive time.

Indeed, if $x \in \partial \Omega \cap U^{*}$, then $\gamma$ solves $\gamma^{\prime}(t)=-\nabla_{T} \Phi(\gamma(t))$ (with $\gamma(0)=x$ ). Since the function $t \mapsto \Phi(\gamma(t))$ is decreasing, the curve $\gamma$ remains in $\partial \Omega \cap U^{*}=\partial \Omega \cap \Phi^{-1}([0, \varepsilon))$ and is defined on $\mathbb{R}_{+}$. Moreover, $\lim _{t \rightarrow+\infty} \gamma(t)=x^{*}$.

Besides, if $x \in \Omega \cap U^{*}$, let us introduce the first time $t_{\partial \Omega}$ such that $\gamma\left(t_{\partial \Omega}\right) \notin \Omega \cap U^{*}$ for the curve solution to $\gamma^{\prime}(t)=-\nabla \Phi\left(\gamma(t)\right.$ ) (with $\gamma(0)=x$ ). If $t_{\partial \Omega}=\infty$, then $\gamma$ belongs to $U^{*} \cap \Omega$ for all time, and since $t \mapsto \Phi(\gamma(t))$ is decreasing, necessarily, $\lim _{t \rightarrow+\infty} \gamma(t)=x^{*}$. If $t_{\partial \Omega}<\infty$, then, since $t \mapsto \Phi(\gamma(t))$ is decreasing and $U^{*}=\Phi^{-1}([0, \varepsilon))$, necessarily, 
$\gamma\left(t_{\partial \Omega}\right) \in \partial \Omega \cap U^{*}$. The curve $\gamma$ is then defined on $\left[t_{\partial \Omega}, \infty\right)$ as above, for an initial condition in $\partial \Omega \cap U^{*}$.

We have thus shown that the function $\gamma$ is globally defined, piecewise $C^{\infty}$, continuous, remains in $\partial \Omega$ if it enters $\partial \Omega$, and satisfies

$$
\lim _{t \rightarrow+\infty} \gamma(t)=x^{*}
$$

Recall that $t_{\partial \Omega}=\inf \{t \in[0,+\infty), \gamma(t) \in \partial \Omega\} \in[0, \infty]$. One has

$$
\begin{aligned}
-\Phi(x) & =\int_{0}^{+\infty} \frac{d}{d t} \Phi \circ \gamma(t) d t \\
& =\int_{0}^{t_{\partial \Omega}} \nabla \Phi(\gamma(t)) \cdot \gamma^{\prime}(t) d t+\int_{t_{\partial \Omega}}^{+\infty} \nabla_{T} \Phi(\gamma(t)) \cdot \gamma^{\prime}(t) d t \\
& =-\left(\int_{0}^{t_{\partial \Omega}}|\nabla \Phi(\gamma(t))|^{2} d t+\int_{t_{\partial \Omega}}^{+\infty}\left|\nabla_{T} \Phi(\gamma(t))\right|^{2} d t\right) \\
& =-\left(\int_{0}^{t_{\partial \Omega}}|\nabla \Phi(\gamma(t))|\left|\gamma^{\prime}(t)\right| d t+\int_{t_{\partial \Omega}}^{+\infty}\left|\nabla_{T} \Phi(\gamma(t))\right|\left|\gamma^{\prime}(t)\right| d t\right) \\
& =-\int_{0}^{+\infty} g(\gamma(t))\left|\gamma^{\prime}(t)\right| d t=-\lim _{t \rightarrow+\infty} L(\gamma,(0, t)) .
\end{aligned}
$$

Thanks to Lemma 43 ,

$$
d_{a}\left(x, x^{*}\right) \leq L(\gamma,(0,+\infty))=\Phi(x) .
$$

In conclusion, $\Phi(x)=d_{a}\left(x^{*}, x\right)$ for all $x \in U^{*}$.

Remark 56. Let us mention a simple consequence of the previous proof that will be useful in the following. If $x^{*} \in \partial \Omega$ is such that $\nabla_{T} f\left(x^{*}\right)=0$, there exists a neighborhood $U^{*}$ of $x^{*}$ such that for all $x \in U^{*}$, there exists a piecewise $C^{\infty}$ and continuous curve $\gamma: \mathbb{R}_{+} \rightarrow \bar{\Omega}$ such that

$$
d_{a}\left(x^{*}, x\right)=\int_{0}^{+\infty} g(\gamma(t))\left|\gamma^{\prime}(t)\right| d t
$$

with $\gamma(0)=x$ and $\lim _{t \rightarrow+\infty} \gamma(t)=x^{*}$. The curve $\gamma$ is solution to 96-97). In addition, the set $\partial\{t \in[0, \infty), \gamma(t) \in \partial \Omega\}$ either consists of one point or is empty.

\subsection{Curves realizing the Agmon distance}

In this section, it is proven that for any two points $x \in \bar{\Omega}$ and $y \in \bar{\Omega}$, their exists a finite number of curves $\left(\gamma_{i}\right)_{i=1, \ldots, N}$ defined on the intervals $\left(I_{i}\right)_{i=1, \ldots, N}$ such that the sum of their lengths $L\left(\gamma_{i}, I_{i}\right)$ equals the Agmon distance $d_{a}(x, y)$. The precise statement is given in the following theorem.

Theorem 3. Assume that [H1] and [H3] hold. Let $x, y \in \bar{\Omega}$. Then there exists a finite number of Lipschitz curves $\left(\gamma_{j}\right)_{j=1, \ldots, N}$ which are defined on possibly unbounded intervals $I_{j} \subset \mathbb{R}$, with values in $\bar{\Omega}$, such that for all $j \in\{1, \ldots, N\}$, the sets $\partial\left\{t \in I_{j}, \gamma_{j}(t) \in \partial \Omega\right\}$ are finite and

$$
d_{a}(x, y)=\sum_{j=1}^{N} L\left(\gamma_{j}, I_{j}\right)
$$


Additionally, by construction, the intervals $\left(I_{j}\right)_{j \in\{1, \ldots, N\}}$ are either $[0+\infty),(-\infty, 0]$ or $[0,1]$. Moreover, if $I_{j}=[0,+\infty)$ or $I_{j}=(-\infty, 0]$, then $\gamma_{j}$ is continuous and piecewise $C^{\infty}$ (see Lemma 60 below for a more precise definition of the curves $\gamma_{j}$ in this case). If $I_{j}=[0,1]$, then $\gamma_{j} \in A\left(\gamma_{j}(0), \gamma_{j}(1)\right)$. Finally the curves $\left(\left(\gamma_{1}, I_{1}\right), \ldots,\left(\gamma_{N}, I_{N}\right)\right)$ are ordered such that

$$
\lim _{t \rightarrow\left(\inf I_{1}\right)^{+}} \gamma_{1}(t)=x, \quad \lim _{t \rightarrow\left(\sup I_{N}\right)^{-}} \gamma_{N}(t)=y,
$$

and for all $k \in\{1, \ldots, N-1\}$,

$$
\lim _{t \rightarrow\left(\sup I_{k}\right)^{-}} \gamma_{k}(t)=\lim _{t \rightarrow\left(\inf I_{k+1}\right)^{+}} \gamma_{k+1}(t) .
$$

This section is entirely dedicated to the proof of Theorem 3 . In the following, one denotes by

$$
\left\{x_{1}, \ldots, x_{m}\right\}=\{x \in \bar{\Omega}, g(x)=0\},
$$

where $g$ is defined by (17) (there is a finite number of zeros of the function $g$ thanks to $[\mathbf{H 1}])$.

\subsubsection{Preliminary results}

Let us first consider the simple case when the curve realizing the Agmon distance does not meet zeros of the function $g$.

Lemma 57. Assume that [H1] and [H3] hold. Let $(x, y) \in \bar{\Omega} \times \bar{\Omega}$. Let $\left(\gamma_{n}\right)_{n \geq 0} \in$ $A(x, y)^{\mathbb{N}}$ be a minimizing sequence of curves for $d_{a}(x, y): \lim _{n \rightarrow \infty} L\left(\gamma_{n},(0,1)\right)=d_{a}(x, y)$. In addition, assume that for each $k \in\{1, \ldots, m\}$, there exists a neighborhood $V_{k}$ of $x_{k}$ in $\bar{\Omega}$, such that:

$$
\forall n \in \mathbb{N}, \forall k \in\{1, \ldots, m\}, \operatorname{Ran}\left(\gamma_{n}\right) \cap V_{k}=\emptyset .
$$

Then, there exists $\gamma \in A(x, y)$ such that

$$
L(\gamma,(0,1))=d_{a}(x, y) .
$$

Proof. Let $M$ be such that for all $n, L\left(\gamma_{n},(0,1)\right) \leq M$ and let us define

$$
c:=\inf _{\bar{\Omega} \backslash\left(V_{1} \cup \ldots \cup V_{m}\right)} g>0 .
$$

One defines for $t \in[0,1], \phi_{n}(t)=\frac{L\left(\gamma_{n},(0, t)\right)+t}{L\left(\gamma_{n},(0,1)\right)+1}$. The map $\phi_{n}$ is strictly increasing and continuous from $[0,1]$ to $[0,1]$. Therefore it admits an inverse. Setting $\tilde{\gamma}_{n}(u):=$ $\gamma_{n} \circ \phi_{n}^{-1}(u)$, one gets $L\left(\gamma_{n},(0,1)\right)=L\left(\tilde{\gamma}_{n},(0,1)\right)$ and

$$
\begin{aligned}
\left|\tilde{\gamma}_{n}^{\prime}\right|\left(\phi_{n}(t)\right) & =\frac{\left|\gamma_{n}^{\prime}(t)\right|}{g\left(\gamma_{n}(t)\right)\left|\gamma_{n}^{\prime}(t)\right|+1}\left(L\left(\gamma_{n},(0,1)\right)+1\right) \\
& \leq \frac{\left|\gamma_{n}^{\prime}(t)\right|}{c\left|\gamma_{n}^{\prime}(t)\right|+1}\left(L\left(\gamma_{n},(0,1)\right)+1\right) \\
& \leq \frac{1}{c}\left(L\left(\gamma_{n},(0,1)\right)+1\right) \\
& \leq \frac{1}{c}(M+1) .
\end{aligned}
$$

Thus, up to replacing $\gamma_{n}$ by $\tilde{\gamma}_{n}$, one may assume that the Lipchitz constants of $\gamma_{n}$ are bounded uniformly in $n$. In addition since for all $t \in[0,1], \gamma_{n}(t) \in \bar{\Omega}$, the sequence 
$\left(\gamma_{n}\right)_{n \geq 0}$ is relatively compact in $C^{0}([0,1], \bar{\Omega})$. Thus, up to the extraction of a subsequence, there exists a Lipschitz curve $\gamma$ such that $\lim _{n \rightarrow \infty} \gamma_{n}=\gamma$ uniformly on $[0,1]$. Moreover since $\left(\gamma_{n}\right)_{n \geq 0}$ is bounded in $H^{1}([0,1], \bar{\Omega})$, up to the extraction of a subsequence, $\left(\gamma_{n}\right)_{n \geq 0}$ converges weakly to $\gamma$ in $H^{1}([0,1], \bar{\Omega})$. It is not difficult to see that for all $t \in[0,1]$,

$$
\liminf _{n \rightarrow \infty} g\left(\gamma_{n}(t)\right) \geq g(\gamma(t)) .
$$

Indeed, for $t \in[0,1]$, there are two cases:

- If $\gamma(t) \in \Omega$, then for $n$ large enough, all the points $\gamma_{n}(t)$ are in $\Omega$ and thus $\liminf _{n \rightarrow \infty} g\left(\gamma_{n}(t)\right)=\lim _{n \rightarrow \infty} g\left(\gamma_{n}(t)\right)=|\nabla f(\gamma(t))|=g(\gamma(t))$,

- If $\gamma(t) \in \partial \Omega$, since $\mathbb{N}=\left\{n, \gamma_{n}(t) \in \partial \Omega\right\} \cup\left\{n, \gamma_{n}(t) \in \Omega\right\}$, one obtains that the set of limit points of $\left(\left|\nabla f\left(\gamma_{n}(t)\right)\right|\right)_{n \geq 0}$ is included in $\left\{|\nabla f(\gamma(t))|,\left|\nabla_{T} f(\gamma(t))\right|\right\}$. Therefore, from [H3], one has: $\liminf _{n \rightarrow \infty} g\left(\gamma_{n}(t)\right) \geq\left|\nabla_{T} f(\gamma(t))\right|=g(\gamma(t))$.

Then, one obtains

$$
\begin{aligned}
d_{a}(x, y)=\lim _{l \rightarrow \infty} \int_{0}^{1} g\left(\gamma_{l}(t)\right)\left|\gamma_{l}^{\prime}(t)\right| d t & \geq \liminf _{n \rightarrow \infty} \liminf _{p \rightarrow \infty} \int_{0}^{1} g\left(\gamma_{p}(t)\right)\left|\gamma_{n}^{\prime}(t)\right| d t \\
& \geq \liminf _{n \rightarrow \infty} \int_{0}^{1} \liminf _{p \rightarrow \infty} g\left(\gamma_{p}(t)\right)\left|\gamma_{n}^{\prime}(t)\right| d t \\
& \geq \liminf _{n \rightarrow \infty} \int_{0}^{1} g(\gamma(t))\left|\gamma_{n}^{\prime}(t)\right| d t \\
& \geq \int_{0}^{1} g(\gamma(t))\left|\gamma^{\prime}(t)\right| d t .
\end{aligned}
$$

In the previous computation, one used Fatou Lemma and the lower semi continuity (for the weak convergence) of the convex functional

$$
h \in H^{1}([0,1], \bar{\Omega}) \mapsto \int_{0}^{1} g(\gamma(t))\left|h^{\prime}(t)\right| d t .
$$

Since [H3] holds, using Proposition 38 , there exists a curve $\tilde{\gamma} \in A(x, y)$ such that $L(\gamma,(0,1)) \geq L(\tilde{\gamma},(0,1))$ and thus $d_{a}(x, y)=L(\tilde{\gamma},(0,1))$.

Let us now introduce a sufficient condition so that a minimizing sequence of curves realizing the Agmon distance avoids a neighborhood of a zero of the function $g$. For $x \in \bar{\Omega}$, one introduces the following sets:

$$
\forall k \in\{1, \ldots, m\}, A_{k}(x):=\left\{z \in \bar{\Omega}, d_{a}(x, z)=d_{a}\left(x, x_{k}\right)+d_{a}\left(x_{k}, z\right)\right\} .
$$

One notices that $z \in A_{k}(x)$ if and only if $x \in A_{k}(z)$.

Proposition 58. Assume that [H1] and [H3] hold. Let $(x, y) \in \bar{\Omega}^{2}$ and assume that there exists $k \in\{1, \ldots, m\}$ such that $y \notin A_{k}(x)$. If $\left(\gamma_{n}\right)_{n \geq 0} \in A(x, y)^{\mathbb{N}}$ is a minimizing sequence of curves for $d_{a}(x, y)$, then there exists a neighborhood $V_{k}$ of $x_{k}$ in $\bar{\Omega}$ and $n_{0} \in \mathbb{N}$, such that for all $n \geq n_{0}$,

$$
\operatorname{Ran}\left(\gamma_{n}\right) \cap V_{k}=\emptyset .
$$


Proof. If $y \notin A_{k}(x)$, for a $k \in\{1, \ldots, m\}$, then $d_{a}(x, y)<d_{a}\left(x, x_{k}\right)+d_{a}\left(x_{k}, y\right)$ and thus $y \neq x_{k}$ and $x \neq x_{k}$. Let us define

$$
\varepsilon:=d_{a}\left(x, x_{k}\right)+d_{a}\left(x_{k}, y\right)-d_{a}(x, y)>0,
$$

and $V_{k}:=B_{a}\left(x_{k}, \min \left(\frac{\varepsilon}{3}, \frac{d_{a}\left(x_{k}, y\right)}{2}\right)\right)$ where

$$
\forall z \in \bar{\Omega}, \forall r>0, B_{a}(z, r):=\left\{u \in \bar{\Omega}, d_{a}(z, u)<r\right\} .
$$

Notice that $y \notin V_{k}$. We now prove Proposition 58 by contradiction. We assume that, up to the extraction of a subsequence, for all $n \in \mathbb{N}$, $\operatorname{Ran}\left(\gamma_{n}\right) \cap V_{k} \neq \emptyset$. Let us define, for all $n \in \mathbb{N}$,

$$
t_{0}^{n}:=\inf \left\{t \in[0,1], \gamma_{n}(t) \in V_{k}\right\} \text { and } t_{1}^{n}:=\sup \left\{t \in[0,1], \gamma_{n}(t) \in V_{k}\right\} .
$$

We have for all $n \in \mathbb{N}$, owing to the triangular inequality,

$$
L\left(\gamma_{n},\left(0, t_{0}^{n}\right)\right) \geq d_{a}\left(x, x_{k}\right)-\frac{\varepsilon}{3}, \quad L\left(\gamma_{n},\left(t_{1}^{n}, 1\right)\right) \geq d_{a}\left(x_{k}, y\right)-\frac{\varepsilon}{3} .
$$

Thus, for all $n \in \mathbb{N}$, it holds:

$$
\begin{aligned}
L\left(\gamma_{n}(0,1)\right) & \geq L\left(\gamma_{n},\left(0, t_{0}^{n}\right)\right)+L\left(\gamma_{n},\left(t_{1}^{n}, 1\right)\right) \\
& \geq d_{a}\left(x, x_{k}\right)+d_{a}\left(x_{k}, y\right)-\frac{2 \varepsilon}{3}=d_{a}(x, y)+\frac{\varepsilon}{3} .
\end{aligned}
$$

This contradicts the fact that $\lim _{n \rightarrow \infty} L\left(\gamma_{n},(0,1)\right)=d_{a}(x, y)$.

A direct corollary of Proposition 58 and Lemma 57 is the following result:

Corollary 59. Assume that [H1] and [H3] hold. Let $y \in \bar{\Omega}$ and assume that $y \notin A_{j}(x)$ for all $j \in\{1, \ldots, m\}$. Then, there exists a curve $\gamma \in A(x, y)$ such that

$$
d_{a}(x, y)=L(\gamma,(0,1)) .
$$

Notice that $y \notin A_{j}(x)$ for all $j \in\{1, \ldots, m\}$ implies in particular that $x$ and $y$ are not zeros of the function $g$. This corollary will be used below to build the curves $\gamma_{j}$ associated with intervals $I_{j}=[0,1]$ in Theorem 3. The curves $\gamma_{j}$ associated with intervals $I_{j}=[0,+\infty)$ or $I_{j}=(-\infty, 0]$ will be built using the following lemma, which is a direct consequence of Remarks 49 and 56 .

Lemma 60. Assume that [H1] and [H3] hold. Let $k \in\{1, \ldots, m\}$. There exists a neighborhood $V_{k}$ of $x_{k}$ in $\bar{\Omega}$, such that for all $y \in V_{k}$, there exists a continuous and piecewise $C^{\infty}$ curve $\gamma:(-\infty, 0] \rightarrow V_{k}$ satisfying

$$
d_{a}\left(y, x_{k}\right)=L(\gamma,(-\infty, 0]), \lim _{t \rightarrow-\infty} \gamma(t)=x_{k}, \gamma(0)=y .
$$

If $x_{k} \in \Omega, \gamma$ is with values in $\Omega$ and satisfies (90). If $x_{k} \in \partial \Omega, \gamma$ satisfies (96)-(97) and is such that $\partial\{t \in(-\infty, 0], \gamma(t) \in \partial \Omega\}$ is either empty or a single point.

Before proving Theorem 3, we finally need two additional preliminary lemmas.

Lemma 61. Assume that [H1] and [H3] hold. Let $u \in \bar{\Omega}$ and $w \in \bar{\Omega}$. For any $\delta>0$ small enough, there exists $z_{\delta}$ such that $d_{a}\left(u, z_{\delta}\right)=\delta$ and $d_{a}(w, u)=d_{a}\left(w, z_{\delta}\right)+d_{a}\left(z_{\delta}, u\right)$. 
Proof. Notice that $d_{a}\left(u, z_{\delta}\right)=\delta$ is equivalent to $z_{\delta} \in \partial B_{a}(u, \delta)$, where $B_{a}$ is defined by (99). We prove Lemma 61 by contradiction. Assume that there exists $\delta \in\left(0, \frac{d_{a}(u, w)}{2}\right)$ such that for all $z \in \partial B_{a}(u, \delta), d_{a}(w, u)<d_{a}(w, z)+d_{a}(z, u)$. By compactness of $\partial B_{a}(u, \delta)$, there exists $a_{\delta}>0$ such that for all $z \in \partial B_{a}(u, \delta)$,

$$
d_{a}(w, u)+a_{\delta} \leq d_{a}(w, z)+d_{a}(z, u) .
$$

Thus if $\gamma \in A(u, w)$, since there exists a time $t_{\delta}$ such that $\gamma\left(t_{\delta}\right) \in \partial B_{a}(u, \delta)$, one has

$$
\begin{aligned}
L(\gamma,(0,1))=L\left(\gamma,\left(0, \gamma\left(t_{\delta}\right)\right)\right)+L\left(\gamma,\left(\gamma\left(t_{\delta}\right), 1\right)\right) & \geq d_{a}\left(u, \gamma\left(t_{\delta}\right)\right)+d_{a}\left(\gamma\left(t_{\delta}\right), w\right) \\
& \geq d_{a}(u, w)+a_{\delta}
\end{aligned}
$$

This is impossible since by definition $d_{a}(u, w)=\inf _{\gamma \in A(u, w)} L(\gamma,(0,1))$.

Lemma 62. Assume that [H1] holds. Let $(x, y) \in \bar{\Omega}^{2}$ with $x \neq y$. Then, there exist $N \in \mathbb{N}$ and a sequence $\left(b_{i}\right)_{i \in\{0, \ldots, N+1\}} \in \bar{\Omega}^{N+2}, b_{0}=x, b_{N+1}=y,\left(b_{i}\right)_{i \in\{1, \ldots, N\}} \in$ $\left\{x_{1}, \ldots, x_{m}\right\}^{N}$ (with the convention $\left\{x_{1}, \ldots, x_{m}\right\}^{0}=\emptyset$ ) such that the following holds:

1. For all $i \in\{0, \ldots, N\}, b_{i} \neq b_{i+1}$ and

$$
d_{a}(x, y)=\sum_{i=0}^{N} d_{a}\left(b_{i}, b_{i+1}\right) .
$$

2. For all $i \in\{0, \ldots, N\}$ and for all $z \in\left\{x, y, x_{1}, \ldots, x_{m}\right\} \backslash\left\{b_{i}, b_{i+1}\right\}$,

$$
d_{a}\left(b_{i}, b_{i+1}\right)<d_{a}\left(b_{i}, z\right)+d_{a}\left(z, b_{i+1}\right) .
$$

Proof. Since $x \neq y$, the following set

$$
\begin{aligned}
E:=\{ & (N, b), N \in \mathbb{N}, b=\left(b_{i}\right)_{i \in\{0, \ldots, N+1\} \in \bar{\Omega}^{N+2}, b_{0}=x, b_{N+1}=y,} \\
& \left.\forall i \in\{0, \ldots, N\}, b_{i} \neq b_{i+1},\left(b_{i}\right)_{i \in\{1, \ldots, N\}} \in\left\{x_{1}, \ldots, x_{m}\right\}^{N}, 100 \text { holds }\right\},
\end{aligned}
$$

is not empty since by assumption it contains $(0,\{x, y\})$. For $(N, b) \in E$, one defines the cardinal of $(N, b)$ by the number of different critical points $b$ contains. The cardinal of an element of $E$ belongs to $\{0, \ldots, m\}$.

Let us now consider an element $(N, b) \in E$ which is maximal for the cardinal. By construction, this element satisfies point 1 in Lemma 62. Let us now show that it also satisfies point 2 in Lemma 62 . Notice that $\left\{b_{0}, \ldots, b_{N+1}\right\} \subset\left\{x, y, x_{1}, \ldots, x_{m}\right\}$. Let $i \in\{0, \ldots, N\}$ and $z \in\left\{x, y, x_{1}, \ldots, x_{m}\right\} \backslash\left\{b_{i}, b_{i+1}\right\}$. If $z \in\left\{x, y, x_{1}, \ldots, x_{m}\right\} \backslash$ $\left\{b_{0}, \ldots, b_{N+1}\right\}$, the equality $d_{a}\left(b_{i}, b_{i+1}\right)=d_{a}\left(b_{i}, z\right)+d_{a}\left(z, b_{i+1}\right)$ cannot hold since $b$ has been chosen maximal in $E$ for the cardinal. Thus, by the triangular inequality $d_{a}\left(b_{i}, b_{i+1}\right)<d_{a}\left(b_{i}, z\right)+d_{a}\left(z, b_{i+1}\right)$. If $z \in\left\{b_{0}, \ldots, b_{N+1}\right\} \backslash\left\{b_{i}, b_{i+1}\right\}$, let us prove that $d_{a}\left(b_{i}, b_{i+1}\right)<d_{a}\left(b_{i}, z\right)+d_{a}\left(z, b_{i+1}\right)$ by contradiction. By the triangular inequality, if the previous inequality does not hold, one has $d_{a}\left(b_{i}, b_{i+1}\right)=d_{a}\left(b_{i}, z\right)+d_{a}\left(z, b_{i+1}\right)$ for some $z \in\left\{b_{0}, \ldots, b_{N+1}\right\} \backslash\left\{b_{i}, b_{i+1}\right\}$. Let us denote by $j_{0} \in\{0, \ldots, i-1, i+2, \ldots, N+1\}$ the index such that $z=b_{j_{0}}$. One has $d_{a}\left(b_{i}, b_{i+1}\right)=d_{a}\left(b_{i}, b_{j_{0}}\right)+d_{a}\left(b_{j_{0}}, b_{i+1}\right)$. Let us assume 
without loss of generality that $j_{0}<i$ (the case $j_{0}>i+1$ is treated similarly). In this case, one has, using the triangular inequality:

$$
\begin{aligned}
d_{a}(x, y) & =\sum_{j=0}^{N} d_{a}\left(b_{j}, b_{j+1}\right) \\
& =\sum_{j=0}^{i-1} d_{a}\left(b_{j}, b_{j+1}\right)+d_{a}\left(b_{i}, b_{j_{0}}\right)+d_{a}\left(b_{j 0}, b_{i+1}\right)+\sum_{j=i+1}^{N} d_{a}\left(b_{j}, b_{j+1}\right) \\
& =\sum_{j=0}^{j_{0}-1} d_{a}\left(b_{j}, b_{j+1}\right)+d_{a}\left(b_{j_{0}}, b_{i+1}\right)+\sum_{j=i+1}^{N} d_{a}\left(b_{j}, b_{j+1}\right)+\sum_{j=j_{0}}^{i-1} d_{a}\left(b_{j}, b_{j+1}\right)+d_{a}\left(b_{i}, b_{j_{0}}\right) \\
& \geq d_{a}(x, y)+\sum_{j=j_{0}}^{i-1} d_{a}\left(b_{j}, b_{j+1}\right)+d_{a}\left(b_{i}, b_{j_{0}}\right) .
\end{aligned}
$$

Thus, $\sum_{j=j_{0}}^{i-1} d_{a}\left(b_{j}, b_{j+1}\right)+d_{a}\left(b_{i}, b_{j_{0}}\right)=0$ and $b_{j_{0}}=b_{i}$ which is in contradiction with $z \notin\left\{b_{i}, b_{i+1}\right\}$. Therefore $d_{a}\left(b_{i}, b_{i+1}\right)<d_{a}\left(b_{i}, b_{j_{0}}\right)+d_{a}\left(b_{j_{0}}, b_{i+1}\right)$. This concludes the proof of Lemma 62 .

\subsubsection{Proof of Theorem 3}

Let us now prove Theorem 3 . Recall that by assumption, the hypotheses [H1] and [H3] hold.

Proof. Let $x, y \in \bar{\Omega}$. If $x=y$, then Theorem 3 is proved by taking the constant curve $\gamma(t)=x$ for all $t \in[0,1]$. Let us deal with the case $x \neq y$. From Lemma 62, there exist $N \in \mathbb{N}$ and a sequence $\left(b_{j}\right)_{j \in\{0, \ldots, N+1\} \subset} \subset \bar{\Omega}^{N+2}$ such that $b_{0}=x, b_{N+1}=y$,

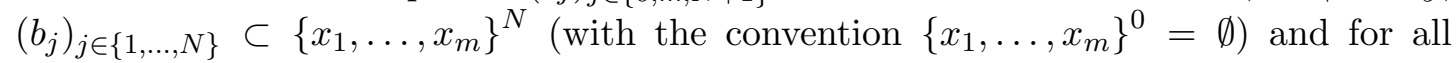
$k \in\{0, \ldots, N\}, b_{k} \neq b_{k+1}$ and

$$
d_{a}(x, y)=\sum_{k=0}^{N} d_{a}\left(b_{k}, b_{k+1}\right) .
$$

If $N=0$, then for all $k \in\{1, \ldots, m\} y \notin A_{k}(x)$ and Theorem 3 is then a consequence of Corollary 59 .

Let us now assume that $N \geq 1$, namely that there exists $k \in\{1, \ldots, m\}$ such that $y \in A_{k}(x)$. Let us actually consider the case $N \geq 2$ (the case $N=1$ is treated similarly). Let $k \in\{1, \ldots, N-1\}$ and let us consider the term $d_{a}\left(b_{k}, b_{k+1}\right)$ in (101) (the first term $d_{a}\left(x, b_{1}\right)$ and the last term $d_{a}\left(b_{N}, y\right)$ in the sum are treated in a similar way). One can label the points $\left\{x_{1}, \ldots, x_{m}\right\}$ such that $b_{k}=x_{1}$ and $b_{k+1}=x_{2}$. Point 2 in Lemma 62 implies that $x_{2} \notin A_{j}\left(x_{1}\right)$ for all $j \in\{3, \ldots, m\}$. From Lemma 61, for any $\delta>0$ there exists $z_{1} \in \partial B_{a}\left(x_{1}, \delta\right)$ such that $d_{a}\left(x_{1}, x_{2}\right)=d_{a}\left(x_{1}, z_{1}\right)+d_{a}\left(z_{1}, x_{2}\right)$ (where $B_{a}$ is defined by (99)). By taking $\delta$ small enough, this implies that $z_{1} \notin A_{1}\left(x_{2}\right)$ and $z_{1} \notin\left\{x_{1}, \ldots, x_{m}\right\}$. Likewise, from Lemma 61, for any $\delta>0$ there exists $z_{2} \in \partial B_{a}\left(x_{2}, \delta\right)$ such that $d_{a}\left(z_{1}, x_{2}\right)=d_{a}\left(z_{1}, z_{2}\right)+d_{a}\left(z_{2}, x_{2}\right)$ and by taking $\delta$ small enough, this implies that $z_{2} \notin A_{2}\left(z_{1}\right)$ and $z_{2} \notin\left\{x_{1}, \ldots, x_{m}\right\}$. Therefore one gets

$$
d_{a}\left(b_{k}, b_{k+1}\right)=d_{a}\left(x_{1}, x_{2}\right)=d_{a}\left(x_{1}, z_{1}\right)+d_{a}\left(z_{1}, z_{2}\right)+d_{a}\left(z_{2}, x_{2}\right) .
$$


Taking $\delta$ small enough and using Lemma 60 , there exists a continuous and piecewise $C^{\infty}$ curve $\gamma_{1}$ defined on $(-\infty, 0]$ such that $d_{a}\left(x_{1}, z_{1}\right)=L\left(\gamma_{1},(-\infty, 0]\right), \lim _{t \rightarrow-\infty} \gamma_{1}(t)=x_{1}$, $\gamma_{1}(0)=z_{1}$, and $\partial\left\{t \in(-\infty, 0], \gamma_{1}(t) \in \partial \Omega\right\}$ is either empty or a single point. Similarly, there exists a continuous and piecewise $C^{\infty}$ curve $\gamma_{2}$ defined on $[0,+\infty)$ such that $d_{a}\left(z_{2}, x_{2}\right)=L\left(\gamma_{2},[0,+\infty)\right), \gamma_{2}(0)=z_{2}, \lim _{t \rightarrow+\infty} \gamma_{2}(t)=x_{2}$ and $\partial\left\{t \in[0,+\infty), \gamma_{2}(t) \in\right.$ $\partial \Omega\}$ is either empty or a single point. Let us show by contradiction that $z_{2} \notin A_{j}\left(z_{1}\right)$ for all $j \in\{3, \ldots, m\}$. On the one hand, if $z_{2} \in A_{j}\left(z_{1}\right)$ for some $j \in\{3, \ldots, m\}$, one has

$$
d_{a}\left(x_{1}, x_{2}\right)=d_{a}\left(x_{1}, z_{1}\right)+d_{a}\left(z_{1}, x_{j}\right)+d_{a}\left(x_{j}, z_{2}\right)+d_{a}\left(z_{2}, x_{2}\right) .
$$

On the other hand, $x_{1} \notin A_{j}\left(x_{2}\right)$, and thus

$d_{a}\left(x_{1}, x_{2}\right)<d_{a}\left(x_{1}, x_{j}\right)+d_{a}\left(x_{j}, x_{2}\right) \leq d_{a}\left(x_{1}, z_{1}\right)+d_{a}\left(z_{1}, x_{j}\right)+d_{a}\left(x_{j}, z_{2}\right)+d_{a}\left(z_{2}, x_{2}\right)$.

This leads to a contradiction. Therefore $z_{2} \notin A_{j}\left(z_{1}\right)$ for all $j \in\{3, \ldots, m\}$. One also has by a similar reasoning that $z_{2} \notin A_{1}\left(z_{1}\right)$. Indeed, If $z_{2} \in A_{1}\left(z_{1}\right)$, then one has on the one hand

$$
d_{a}\left(z_{1}, x_{2}\right)=d_{a}\left(z_{1}, z_{2}\right)+d_{a}\left(z_{2}, x_{2}\right)=d_{a}\left(z_{1}, x_{1}\right)+d_{a}\left(x_{1}, z_{2}\right)+d_{a}\left(z_{2}, x_{2}\right) .
$$

On the other hand, since $z_{1} \notin A_{1}\left(x_{2}\right)$, one has

$$
d_{a}\left(z_{1}, x_{2}\right)<d_{a}\left(z_{1}, x_{1}\right)+d_{a}\left(x_{1}, x_{2}\right) \leq d_{a}\left(z_{1}, x_{1}\right)+d_{a}\left(x_{1}, z_{2}\right)+d_{a}\left(z_{2}, x_{2}\right) .
$$

This leads to a contradiction. In conclusion $z_{2} \notin A_{j}\left(z_{1}\right)$ for all $j \in\{1, \ldots, m\}$. Therefore, from Corollary 59, there exists a curve $\gamma \in A\left(z_{1}, z_{2}\right)$ such that $d_{a}\left(z_{1}, z_{2}\right)=$ $L(\gamma,(0,1))$. In conclusion, we have built three curves $\gamma, \gamma_{1}$ and $\gamma_{2}$ such that

$$
d_{a}\left(b_{k}, b_{k+1}\right)=L\left(\gamma_{1},(-\infty, 0]\right)+L(\gamma,(0,1))+L\left(\gamma_{2},[0,+\infty)\right) .
$$

A similar reasoning for all the terms in the sum in (101) concludes the proof of Theorem 3 .

A consequence of Theorem 3 is the following.

Lemma 63. Let us assume that [H1] and [H3] hold. Let $(x, y) \in \bar{\Omega}$. Let us denote by $\left(\left(\gamma_{1}, I_{1}\right), \ldots,\left(\gamma_{N}, I_{N}\right)\right)$ the curves given by Theorem 3 ordered such that

$$
\lim _{t \rightarrow\left(\inf I_{1}\right)^{+}} \gamma_{1}(t)=x, \quad \lim _{t \rightarrow\left(\sup I_{N}\right)^{-}} \gamma_{N}(t)=y,
$$

and which realize the Agmon distance between $x$ and $y$. Let $k_{1} \leq k_{2}$ with $\left(k_{1}, k_{2}\right) \in$ $\{1, \ldots, N\}^{2}$ and let $t_{1} \in \overline{I_{k_{1}}}$ and $t_{2} \in \overline{I_{k_{2}}}$. If $k_{1}=k_{2}, t_{1}$ and $t_{2}$ are chosen such that $t_{1} \leq t_{2}$. Then, one has:

- If $k_{1}<k_{2}$,

$$
d_{a}\left(\gamma_{k_{1}}\left(t_{1}\right), \gamma_{k_{2}}\left(t_{2}\right)\right)=L\left(\gamma_{k_{1}},\left(t_{1}, \sup I_{k_{1}}\right)\right)+\sum_{k=k_{1}+1}^{k_{2}-1} L\left(\gamma_{k}, I_{k}\right)+L\left(\gamma_{k_{2}},\left(\inf I_{k_{2}}, t_{2}\right)\right),
$$

where by convention, if $k_{2}=k_{1}+1, \sum_{k=k_{1}+1}^{k_{2}-1} L\left(\gamma_{k}, I_{k}\right)=0$. 
- If $k_{1}=k_{2}, d_{a}\left(\gamma_{k_{1}}\left(t_{1}\right), \gamma_{k_{2}}\left(t_{2}\right)\right)=L\left(\gamma_{k_{1}},\left(t_{1}, t_{2}\right)\right)$.

In addition, the following equality holds

$$
d_{a}(x, y)=d_{a}\left(x, \gamma_{k_{1}}\left(t_{1}\right)\right)+d_{a}\left(\gamma_{k_{1}}\left(t_{1}\right), \gamma_{k_{2}}\left(t_{2}\right)\right)+d_{a}\left(\gamma_{k_{2}}\left(t_{2}\right), y\right) .
$$

The proof of Lemma 63 is done easily reasoning by contradiction and using the triangular inequality on the Agmon distance.

\subsubsection{On the equality in $(78)$}

We end up this section with some results in case of equality in the inequality (78). We will prove in particular Proposition 66 which has been used in Section 3.2 .2 above to give lower bounds on the Agmon distance.

Corollary 64. Let us assume that [H1] and [H3] hold. Let $x, y \in \bar{\Omega}$ with $f(x) \leq f(y)$. Let us denote by $\left(\left(\gamma_{1}, I_{1}\right), \ldots,\left(\gamma_{N}, I_{N}\right)\right)$ the curves given by Theorem 3 ordered such that

$$
\lim _{t \rightarrow\left(\inf I_{1}\right)^{+}} \gamma_{1}(t)=x, \quad \lim _{t \rightarrow\left(\sup I_{N}\right)^{-}} \gamma_{N}(t)=y,
$$

and which realize the Agmon distance between $x$ and $y$. If it holds:

$$
d_{a}(x, y)=f(y)-f(x)
$$

then for all $i \in\{1, \ldots, N\}$, there exist measurable functions $\lambda_{i}: I_{i} \rightarrow \mathbb{R}_{+}$such that for almost every $t$ in $\left\{t \in I_{i}, \gamma_{i}(t) \in \Omega\right\}$

$$
\gamma_{i}^{\prime}(t)=\lambda_{i}(t) \nabla f\left(\gamma_{i}(t)\right)
$$

and such that for almost every $t$ in int $\left\{t \in I_{i}, \gamma_{i}(t) \in \partial \Omega\right\}$

$$
\gamma_{i}^{\prime}(t)=\lambda_{i}(t) \nabla_{T} f\left(\gamma_{i}(t)\right)
$$

Moreover, for all $i \in\{1, \ldots, N\}, \lambda_{i} \in L^{\infty}\left(I_{i}, \mathbb{R}_{+}\right)$. Finally, if $I_{i}$ is unbounded (i.e. $I_{i}=[0,+\infty)$ or $\left.I_{i}=(-\infty, 0]\right)$, it holds for almost every $t \in I_{i}, \lambda_{i}=1$.

Proof. Using Lemma 34, one gets using first the triangular inequality and then the Cauchy-Schwarz inequality

$$
\begin{aligned}
f(y)-f(x) & =\sum_{k=1}^{N}\left(\int_{\left\{t \in I_{k}, \gamma_{k}(t) \in \Omega\right\}}(\nabla f)\left(\gamma_{k}\right) \cdot \gamma_{k}^{\prime}+\int_{\operatorname{int}\left\{t \in I_{k}, \gamma_{k}(t) \in \partial \Omega\right\}}\left(\nabla_{T} f\right)\left(\gamma_{k}\right) \cdot \gamma_{k}^{\prime}\right) \\
& \leq \sum_{k=1}^{N}\left(\int_{\left\{t \in I_{k}, \gamma_{k}(t) \in \Omega\right\}}\left|\nabla f\left(\gamma_{k}\right)\right|\left|\gamma_{k}^{\prime}\right|+\int_{\operatorname{int}\left\{t \in I_{k}, \gamma_{k}(t) \in \partial \Omega\right\}}\left|\nabla_{T} f\left(\gamma_{k}\right)\right|\left|\gamma_{k}^{\prime}\right|\right) \\
& =\sum_{k=1}^{N} L\left(\gamma_{k}, I_{k}\right)=d_{a}(x, y) .
\end{aligned}
$$

If $d_{a}(x, y)=f(y)-f(x)$, then the previous inequality is necessarily an equality. Using the cases of equality in both the triangular inequality and the Cauchy-Schwarz inequalities, for $k \in\{1, \ldots, N\}$, there exists a nonnegative function $\lambda_{k}: I_{k} \rightarrow \mathbb{R}_{+}$such that 102 and (103) hold. 
Let $i \in\{1, \ldots, N\}$. Let us first consider the case when $I_{i}=[0,1]$. Then, by construction, the curve $\gamma_{i}$ does not meet any critical points of the functions $f$ and $\left.f\right|_{\partial \Omega}$. This implies that $\inf _{I_{i}}\left|\nabla f\left(\gamma_{i}\right)\right|>0$ and $\inf _{I_{i}}\left|\nabla_{T} f\left(\gamma_{i}\right)\right|>0$, and thus, since $\left\|\gamma_{i}^{\prime}\right\|_{L^{\infty}}<$ $\infty$, one concludes that $\lambda_{i} \in L^{\infty}\left([0,1], \mathbb{R}_{+}\right)$.

Let us now consider the case when $I_{i}$ is not bounded. Using the construction of the curves $\left(\gamma_{k}\right)_{k=1, \ldots, N}$, this implies that $I_{i}$ is either $(-\infty, 0]$ or $[0,+\infty)$ and $\gamma_{i}$ is constructed using the gradient flow of the eikonal solution near a critical point $x^{*}$ of $f$ or of $\left.f\right|_{\partial \Omega}$ (see Lemma 60). Let us assume that $x^{*}$ is a critical point of $\left.f\right|_{\partial \Omega}$ and $I_{i}=[0,+\infty$ ) (the other cases are treated similarly). Let $\Phi$ be the solution to $(93)$ on the neighborhood $V^{*}$ of $x^{*}$ in $\bar{\Omega}$ (see Proposition 53). The curve $\gamma_{i}$ satisfies by construction, $\operatorname{Ran}\left(\gamma_{i}\right) \subset V^{*}$, $\lim _{t \rightarrow \infty} \gamma_{i}(t)=x^{*}$, and on $(-\infty, 0]$,

$$
\gamma_{i}^{\prime}=\left\{\begin{array}{l}
-\nabla \Phi\left(\gamma_{i}\right) \text { in } \Omega \\
-\nabla_{T} \Phi\left(\gamma_{i}\right) \text { on } \partial \Omega
\end{array}\right.
$$

In addition, by the previous reasoning, one also has on $(-\infty, 0]$,

$$
\gamma_{i}^{\prime}=\left\{\begin{array}{l}
\lambda_{i} \nabla f\left(\gamma_{i}\right) \text { in } \Omega, \\
\lambda_{i} \nabla_{T} f\left(\gamma_{i}\right) \text { on } \partial \Omega,
\end{array}\right.
$$

for some measurable function $\lambda_{i}:[0,+\infty) \rightarrow \mathbb{R}_{+}$. Furthermore, from the last point in Proposition 53, it holds on $V^{*} \cap \partial \Omega$,

$$
\left|\nabla_{T} \Phi\right|=\left|\nabla_{T} f\right|
$$

Taking the norm in (104) and (105), and using the fact that $\Phi$ solves 93 together with the equality (106), one obtains that $\lambda_{i}(t)=1$ for almost every $t \in I_{i}$. This concludes the proof of Corollary 64 .

Let us define the notion of generalized integral curves.

Definition 65. Let $D \subset \bar{\Omega}$ be a $C^{\infty}$ domain and $X \in C^{\infty}(D, \mathbb{R})$. Let $N \in \mathbb{N}^{*}$ and for $i \in\{1, \ldots, N\}$, let $I_{i} \subset \mathbb{R}$ be an interval and $\gamma_{i}: I_{i} \rightarrow D$ be Lipschitz and such that

$$
\lim _{t \rightarrow\left(\inf I_{1}\right)^{+}} \gamma_{1}(t) \quad \text { and } \lim _{t \rightarrow\left(\sup I_{N}\right)^{-}} \gamma_{N}(t) \text { exist }
$$

and for all $k \in\{1, \ldots, N-1\}$,

$$
\lim _{t \rightarrow\left(\sup I_{k}\right)^{-}} \gamma_{k}(t)=\lim _{t \rightarrow\left(\inf I_{k+1}\right)^{+}} \gamma_{k+1}(t)
$$

The set of curves $\left\{\gamma_{1}, \ldots, \gamma_{N}\right\}$ is a generalized integral curve of the vector field

$$
\left\{\begin{array}{c}
\nabla X \text { in } D \cap \Omega, \\
\nabla_{T} X \text { on } D \cap \partial \Omega,
\end{array}\right.
$$

if for all $i \in\{1, \ldots, N\}$, there exist measurable functions $\lambda_{i}: I_{i} \rightarrow \mathbb{R}_{+}$such that for almost every $t$ in $\left\{t \in I_{i}, \gamma_{i}(t) \in D \cap \Omega\right\}: \gamma_{i}^{\prime}(t)=\lambda_{i}(t) \nabla X\left(\gamma_{i}(t)\right)$, and such that for almost every $t$ in $\operatorname{int}\left\{t \in I_{i}, \gamma_{i}(t) \in \partial \Omega \cap D\right\}: \gamma_{i}^{\prime}(t)=\lambda_{i}(t) \nabla_{T} X\left(\gamma_{i}(t)\right)$. 
The notion of generalized integral curve has been introduced in the case of manifolds without boundary in [43]. As introduced in Definition 65, the set of curves $\left\{\gamma_{1}, \ldots, \gamma_{N}\right\}$ given by Corollary 64 is a generalized integral curve of the vector field

$$
\left\{\begin{array}{c}
\nabla f \text { in } \Omega, \\
\nabla_{T} f \text { on } \partial \Omega .
\end{array}\right.
$$

Let us mention that in the case when $\Omega$ is a manifold without boundary, Corollary 64 is exactly [43, Lemma A2.2].

Let us end this section with the following proposition which which has been used in Section 3.2.2,

Proposition 66. Let us assume that [H1] and [H3] hold. Let us denote by $\left\{z_{1}, \ldots, z_{n}\right\}$ the local minima of $\left.f\right|_{\partial \Omega}$ ordered such that $f\left(z_{1}\right) \leq f\left(z_{2}\right) \leq \ldots \leq f\left(z_{n}\right)$. Then, for all $i<j,(i, j) \in\{1, \ldots, n\}^{2}$, one has

$$
d_{a}\left(z_{i}, z_{j}\right)>f\left(z_{j}\right)-f\left(z_{i}\right)
$$

Proof. From the inequality (78), one has $d_{a}\left(z_{i}, z_{j}\right) \geq f\left(z_{j}\right)-f\left(z_{i}\right)$ for all $i<j$. Let us prove Proposition 66 by contradiction. Assume that $d_{a}\left(z_{i}, z_{j}\right)=f\left(z_{j}\right)-f\left(z_{i}\right)$ for some $i<j$. Denote by $\left(\left(\gamma_{1}, I_{1}\right), \ldots,\left(\gamma_{m}, I_{m}\right)\right)$ the curves given by Theorem 3 ordered such that

$$
\lim _{t \rightarrow\left(\inf I_{1}\right)^{+}} \gamma_{1}(t)=z_{i}, \quad \lim _{t \rightarrow\left(\sup I_{m}\right)^{-}} \gamma_{m}(t)=z_{j},
$$

and which realize the Agmon distance between $z_{i}$ and $z_{j}$. Since $d_{a}\left(z_{i}, z_{j}\right)=f\left(z_{j}\right)-f\left(z_{i}\right)$, from Corollary 64, for all $i \in\{1, \ldots, m\}$, there exist measurable functions $\lambda_{i}: I_{i} \rightarrow \mathbb{R}_{+}$ such that for almost every $t$ in $\left\{t \in I_{i}, \gamma_{i}(t) \in \Omega\right\}, \gamma_{i}^{\prime}(t)=\lambda_{i}(t) \nabla f\left(\gamma_{i}(t)\right)$, and such that for almost every $t$ in int $\left\{t \in I_{i}, \gamma_{i}(t) \in \partial \Omega\right\}, \gamma_{i}^{\prime}(t)=\lambda_{i}(t) \nabla_{T} f\left(\gamma_{i}(t)\right)$. Let us recall that from Remark 56, $I_{1}=(-\infty, 0]$ and $I_{m}=[0,+\infty)$ since $z_{i}$ and $z_{j}$ are critical points of $\left.f\right|_{\partial \Omega}$.

Step 1. Let us show that for all $t \in(-\infty, 0], \gamma_{1}(t) \in \partial \Omega$.

On the one hand, from Remark 56, $\lim _{t \rightarrow-\infty} \gamma_{1}(t)=z_{i}$ and on $(-\infty, 0]$,

$$
\gamma_{1}^{\prime}=\left\{\begin{array}{l}
\nabla \Phi\left(\gamma_{1}\right) \text { in } \Omega \\
\nabla_{T} \Phi\left(\gamma_{1}\right) \text { on } \partial \Omega,
\end{array}\right.
$$

where $\Phi$ solves 93$)$. On the other hand, from Corollary 64, one has on $(-\infty, 0]$,

$$
\gamma_{1}^{\prime}(t)=\left\{\begin{array}{l}
\nabla f\left(\gamma_{1}\right) \text { in } \Omega \\
\nabla_{T} f\left(\gamma_{1}\right) \text { on } \partial \Omega
\end{array}\right.
$$

Then, for all $t \leq 0$, one has $\frac{d}{d t}\left(f\left(\gamma_{1}\right)(t)-\Phi\left(\gamma_{1}(t)\right)\right)=0$. Therefore there exists $C>0$ such that for all $t \in(-\infty, 0], \gamma_{1}(t) \in\{x, f(x)-\Phi(x)=C\}$. Since $\lim _{t \rightarrow-\infty} \gamma_{1}(t)=$ $z_{i}$ and $(f-\Phi)\left(z_{i}\right)=f\left(z_{i}\right)$, one gets that $C=f\left(z_{i}\right)$ and thus for all $t \in(-\infty, 0]$, $\gamma_{1}(t) \in\left\{x, f(x)-\Phi(x)=f\left(z_{i}\right)\right\}$. From Corollary 54 and Proposition 55, $\gamma_{1}$ lives in a neighborhood $U^{*}$ of $z_{i}$ such that (see Equation $(95)$ ):

$$
\left\{x, f(x)-\Phi(x)=f\left(z_{i}\right)\right\} \subset \partial \Omega .
$$


We thus get that for all $t \leq 0, \gamma_{1}(t) \in \partial \Omega$, and then $\gamma_{1}^{\prime}(t)=\nabla_{T} f\left(\gamma_{1}(t)\right)=\nabla_{T} \Phi\left(\gamma_{1}(t)\right)$. Step 1 is proved.

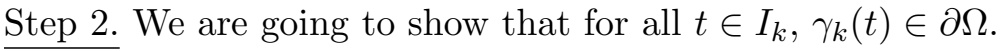

If it is not the case, from Step 1, there exist $k \in\{2, \ldots, m\}$ and $t_{k} \in I_{k}$ such that $\gamma_{k}\left(t_{k}\right) \in$ $\Omega$. Let us define the first time, denoted by $t^{*}$, for which the curves $\left(\left(\gamma_{2}, I_{2}\right), \ldots,\left(\gamma_{m}, I_{m}\right)\right)$ leave $\partial \Omega$. By construction of the curves $\gamma_{1}, \ldots, \gamma_{m}$, there are two cases: either $t^{*}$ is finite (and thus belongs to $\operatorname{int}\left(I_{k}\right)$ for $k \in\{1, \ldots, m\}$ ) or, there exist $j \in\{2, \ldots, m-1\}$, $s<0$ and $z \in \partial \Omega$ such that $g(z)=0, \lim _{t \rightarrow+\infty} \gamma_{j}(t)=z, \lim _{t \rightarrow-\infty} \gamma_{j+1}(t)=z$ and $\gamma_{j+1}(-\infty, s) \subset \Omega$ in which case $t^{*}=-\infty$. Let us assume that $t^{*}$ is finite and belongs to $\operatorname{int}\left(I_{k}\right)$ (the other case is treated similarly).

As in Step 1 of the proof of Proposition 36, let us now introduce a smooth tangential and normal system of coordinates around $\gamma_{k}\left(t^{*}\right)$ in $\bar{\Omega}$, denoted by $\phi(x)=\left(x_{T}, x_{N}\right)$. The function $\phi$ is defined from a neighborhood of $\gamma_{k}\left(t^{*}\right)$ in $\bar{\Omega}$ to $\mathbb{R}^{d}$. Moreover, one has $x_{N} \geq 0$ and $x_{N}(x)=0$ if and only if $x \in \partial \Omega$. We may assume that the neighborhood $V_{\alpha} \subset \mathbb{R}^{d}$ on which $\phi$ is defined is such that $\phi\left(V_{\alpha}\right)=U \times[0, \alpha]$ for $\alpha>0$ and $U \subset \mathbb{R}^{d-1}$. Since $\partial_{n} f>0$ on $\partial \Omega, \alpha>0$ can be chosen small enough such that $\nabla f(x) \cdot n(x)>0$ for all $x \in V_{\alpha}$ where $n(x)=-\frac{\nabla x_{N}(x)}{\left|\nabla x_{N}(x)\right|}$. Indeed, for $x \in \partial \Omega, n(x)$ is nothing but the unit outward normal to $\partial \Omega$.

Now, by continuity of the curve $\gamma_{k}$, there exists $\varepsilon>0$ such that $\left[t^{*}, t^{*}+\varepsilon\right] \subset I_{k}$ and for all $t \in\left[t^{*}, t^{*}+\varepsilon\right], \gamma_{k}(t) \in V_{\alpha}$. The mapping $t \in\left[t^{*}, t^{*}+\varepsilon\right] \mapsto x_{N}\left(\gamma_{k}(t)\right)$ is Lipschitz and satisfies: for almost every $s \in\left(t^{*}, t^{*}+\varepsilon\right)$,

$$
\frac{d}{d s} x_{N}\left(\gamma_{k}(s)\right)=-\left|\nabla x_{N}\left(\gamma_{k}(s)\right)\right| \gamma_{k}^{\prime}(s) \cdot n\left(\gamma_{k}(s)\right) .
$$

Then, for all $t \in\left[t^{*}, t^{*}+\varepsilon\right]$, one has:

$\frac{d}{d s} x_{N}\left(\gamma_{k}(s)\right)=\left\{\begin{array}{l}0 \text { for a.e. } s \in \operatorname{int}\left\{u \in\left(t^{*}, t\right), \gamma_{k}(u) \in \partial \Omega\right\} \\ -\left|\nabla x_{N}\left(\gamma_{k}(s)\right)\right| \lambda_{k}(s) \nabla f\left(\gamma_{k}(s)\right) \cdot n\left(\gamma_{k}(s)\right) \text { for a.e. } s \in\left\{u \in\left(t^{*}, t\right), \gamma_{k}(u) \in \Omega\right\} .\end{array}\right.$

Since $\partial\left\{u \in\left(t^{*}, t\right), \gamma_{k}(u) \in \partial \Omega\right\}$ is of Lebesgue measure zero (see Theorem 3 ) and since $\nabla f \cdot n>0$ in $V_{\alpha}$, one has from Lemma 34, for all $t \in\left[t^{*}, t^{*}+\varepsilon\right]$

$$
x_{N}\left(\gamma_{k}(t)\right)=x_{N}\left(\gamma_{k}(t)\right)-x_{N}\left(\gamma_{k}\left(t^{*}\right)\right)=\int_{t^{*}}^{t} \frac{d}{d s} x_{N}\left(\gamma_{k}(s)\right) d s \leq 0 .
$$

This implies that for all $t \in\left[t^{*}, t^{*}+\varepsilon\right], x_{N}\left(\gamma_{k}(t)\right)=0$ and thus $\gamma_{k}(t) \in \partial \Omega$ for all $t \in\left[t^{*}, t^{*}+\varepsilon\right]$. This contradicts the definition of $t^{*}$. Step 2 is proved.

Step 3. End of the proof of Proposition 66 .

From the last two steps, for all $t \in[0,+\infty), \gamma_{m}(t) \in \partial \Omega$. From Corollary 64, one has $\gamma_{m}^{\prime}(t)=\nabla_{T} f\left(\gamma_{m}(t)\right)$ for all $t \in[0,+\infty)$ and therefore, the map $t \in[0,+\infty) \mapsto f\left(\gamma_{m}(t)\right)$ is increasing (indeed, one has $\left.\frac{d}{d t} f\left(\gamma_{m}(t)\right)=\left|\nabla_{T} f\left(\gamma_{m}(t)\right)\right|^{2}\right)$. This is impossible since $z_{j}$ is a local minimum of $\left.f\right|_{\partial \Omega}$. This concludes the proof of Proposition 66 by contradiction. 


\subsection{Agmon distance in a neighborhood of the basin of attraction of a local minimum of $\left.f\right|_{\partial \Omega}$ and eikonal equation}

The aim of this section is to generalize the results of Section 3.3 to relate the Agmon distance and the solution to an eikonal equation on a neighborhood of a basin of attraction $B_{z}$ (see Definition 9) of a local minimum $z$ of $\left.f\right|_{\partial \Omega}$. Let first introduce a solution to the eikonal equation $|\nabla \phi|^{2}=|\nabla f|^{2}$ defined globally on a neighborhood of the boundary $\partial \Omega$.

Proposition 67. Let us assume that [H3] holds. There exists a neighborhood of $\partial \Omega$ in $\bar{\Omega}$, denoted $V_{\partial \Omega}$, such that there exists $\Phi \in C^{\infty}\left(V_{\partial \Omega}, \mathbb{R}\right)$ satisfying

$$
\left\{\begin{aligned}
|\nabla \Phi|^{2} & =|\nabla f|^{2} \text { in } \Omega \cap V_{\partial \Omega} \\
\Phi & =f \text { on } \partial \Omega \\
\partial_{n} \Phi & =-\partial_{n} f \text { on } \partial \Omega .
\end{aligned}\right.
$$

Moreover, one has the following uniqueness results: if $\tilde{\Phi}$ is a $C^{\infty}$ real valued function defined on a neighborhood $\tilde{V}$ of $\partial \Omega$ satisfying (107), then $\tilde{\Phi}=\Phi$ on $\tilde{V} \cap V_{\partial \Omega}$.

Proof. Let $z \in \partial \Omega$. Using [25] or [28], thanks to [H3], there exists a neighborhood of $z$ in $\bar{\Omega}$, denoted by $\mathcal{V}_{z}$, such that there exists $\Phi \in C^{\infty}\left(\mathcal{V}_{z}, \mathbb{R}\right)$ satisfying

$$
\left\{\begin{aligned}
|\nabla \Phi|^{2} & =|\nabla f|^{2} \text { in } \Omega \cap \mathcal{V}_{z} \\
\Phi & =f \text { on } \partial \Omega \cap \mathcal{V}_{z} \\
\partial_{n} \Phi & =-\partial_{n} f \text { on } \partial \Omega \cap \mathcal{V}_{z} .
\end{aligned}\right.
$$

Moreover, $\mathcal{V}_{z}$ can be chosen such that the following uniqueness result holds: if a function $\tilde{\Phi} \in C^{\infty}\left(\mathcal{V}_{z}, \mathbb{R}\right)$ satisfies the previous equalities, then $\tilde{\Phi}=\Phi$ on $\mathcal{V}_{z}$. Now, one concludes using the fact that $\partial \Omega$ is compact and can thus be covered by a finite number of these neighborhoods $\left(\mathcal{V}_{z}\right)_{z \in \partial \Omega}$.

Remark 68. Let us mention another standard approach to prove Proposition 67, using the notion of viscosity solutions. Let us recall some results from [57, Theorem 5.1]. For $(x, y) \in \bar{\Omega}^{2}$, one defines

$$
\tilde{d}(x, y):=\inf _{T>0, \gamma} \int_{0}^{T}|\nabla f(\gamma(t))| d t
$$

where the infimum is taken over $T>0$ and over Lipschitz curves $\gamma:[0, T] \rightarrow \bar{\Omega}$ which satisfy $\gamma(0)=x, \gamma(T)=y,\left|\gamma^{\prime}\right| \leq 1$. Then, $v(x):=\inf \{f(y)+\tilde{d}(x, y), y \in \partial \Omega\}$ is Lipschitz and is a viscosity solution to

$$
\left\{\begin{aligned}
|\nabla v| & =|\nabla f| \text { in } \Omega \\
v & =f \text { on } \partial \Omega .
\end{aligned}\right.
$$

Let us notice that this implies $\left|\partial_{n} v\right|=\left|\partial_{n} f\right|$ on $\partial \Omega$. To prove Proposition 67 using this result, one has to show that $v$ is $C^{\infty}$ near $\partial \Omega$ and $\partial_{n} v=-\partial_{n} f$. This is a consequence of the characteristic method, see [57, Section 1.2]. 
Remark 69. Let $x^{*}$ be a local minimum of $\left.f\right|_{\partial \Omega}$ and let us denote by $\tilde{\Phi}$ the solution to the eikonal equation (94) introduced in Corollary 54, defined on a neighborhood $V^{*}$ of $x^{*}$. Then, one has on $V^{*} \cap V_{\partial \Omega}$ :

$$
\tilde{\Phi}=\Phi-f\left(x^{*}\right)
$$

where $\Phi$ is the solution to 107 .

Let us now introduce the function $f_{-}$which will be used in the sequel.

Proposition 70. Assume that [H3] holds. Let $\Phi \in C^{\infty}\left(V_{\partial \Omega}, \mathbb{R}\right)$ be the function introduced in Proposition 67. Let us define the function $f_{-} \in C^{\infty}\left(V_{\partial \Omega}, \mathbb{R}\right)$ by

$$
f_{-}=\frac{\Phi-f}{2} .
$$

Then, $f_{-}=0$ on $\partial \Omega$, and up to choosing a smaller neighborhood $V_{\partial \Omega}$ of $\partial \Omega$, the function $f_{-}$is positive in $V_{\partial \Omega} \backslash \partial \Omega$ and $\left|\nabla f_{-}\right|>0$ on $V_{\partial \Omega}$.

Proof. Since $\partial_{n}(\Phi-f)=-2 \partial_{n} f<0$ and $\Phi=f$ on $\partial \Omega$, then, up to choosing a smaller neighborhood $V_{\partial \Omega}$ of $\partial \Omega$, one has $\Phi>f$ on $V_{\partial \Omega} \backslash \partial \Omega$ and $|\nabla(\Phi-f)|>0$ on $V_{\partial \Omega}$.

We are now in position to prove the main result of this section.

Proposition 71. Let us assume that [H1] and [H3] hold. Let $\Phi$ be the function given by Proposition 67. Denote by $z$ a local minimum of $\left.f\right|_{\partial \Omega}$ and denote by $B_{z} \subset \partial \Omega$ the associated basin of attraction (see Definition 9). Besides, let $\Gamma_{z} \subset \partial \Omega$ be an open domain such that $\overline{\Gamma_{z}} \subset B_{z}$ and $z \in \Gamma_{z}$. Then there exists a neighborhood of $\overline{\Gamma_{z}}$ in $\bar{\Omega}$, denoted by $V_{\Gamma_{z}}$, such that $\overline{\partial V_{\Gamma_{z}} \cap \partial \Omega} \subset B_{z}$ and for all $x \in V_{\Gamma_{z}}$,

$$
d_{a}(x, z)=\Phi(x)-f(z) .
$$

Notice that in this proposition, $\Gamma_{z}$ can be chosen as large as needed in $B_{z}$.

Proof. Let $\Phi$ be the function given by Proposition 67. The proof is divided into three steps.

Step 1. Let us first define $V_{\Gamma_{z}}$.

To this end let us denote by $f_{-}$and $V_{\partial \Omega}$ respectively the function and the neighborhood of $\partial \Omega$ given by Proposition 70 . For $\varepsilon>0$ small enough one defines

$$
V_{\varepsilon}=\left\{y \in \Omega, 0 \leq f_{-}(y) \leq \varepsilon\right\} \subset V_{\partial \Omega} .
$$

The parameter $\varepsilon>0$ can be chosen such that there is no critical point of $f$ on $\partial V_{\varepsilon} \cap \Omega=$ $\left\{y \in \Omega, f_{-}(y)=\varepsilon\right\}$. The set $V_{\varepsilon}$ is a neighborhood of $\partial \Omega$ in $\bar{\Omega}$ (see Figure 8 for a schematic representation). Let us now fix such a $\varepsilon>0$. Assumption [H3] together with the fact that $\partial_{n} \Phi<0$ on $\partial \Omega$, imply that there exists a neighborhood $V_{\Gamma_{z}}$ of $\overline{\Gamma_{z}}$ in $\bar{\Omega}$, such that $V_{\Gamma_{z}} \subset V_{\varepsilon}, \overline{\partial V_{\Gamma_{z}} \cap \partial \Omega} \subset B_{z}$ and

$$
\partial_{n} \Phi>0, \text { on } \partial V_{\Gamma_{z}} \cap \Omega \text {. }
$$

The set $V_{\Gamma_{z}}$ is schematically represented on Figure 9 . 


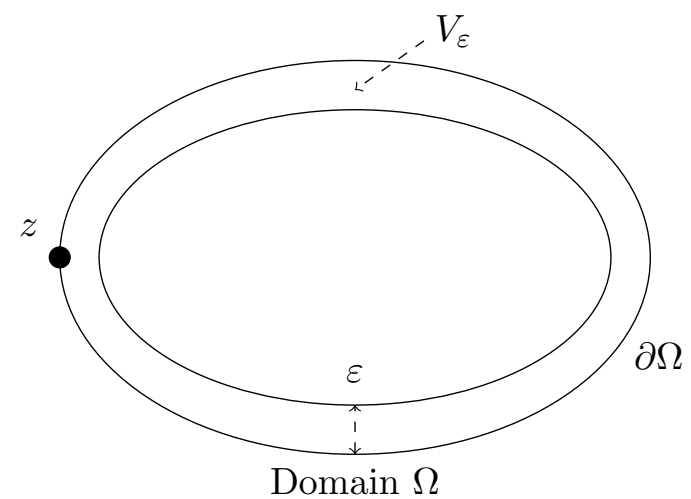

Figure 8: The set $V_{\varepsilon}$.

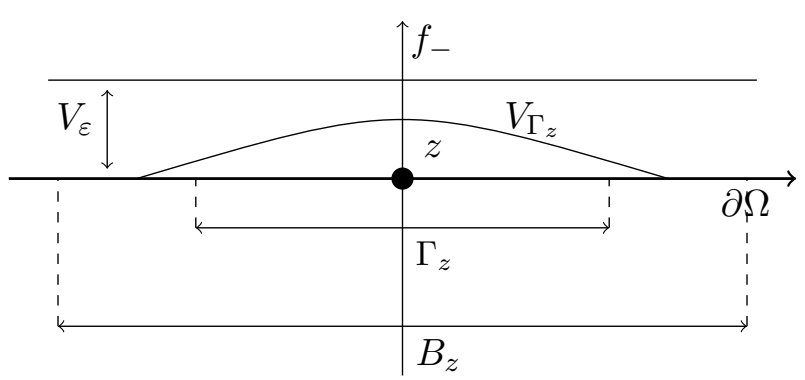

Figure 9: The set $V_{\Gamma_{z}}$.

Step 2. Let us first prove that for all $x \in V_{\Gamma_{z}}, d_{a}(x, z) \geq \Phi(x)-f(z)$.

For $x \in V_{\Gamma_{z}}$, denote by $\left(\left(\gamma_{1}, I_{1}\right), \ldots,\left(\gamma_{N}, I_{N}\right)\right)$ the curves given by Theorem 3 ordered such that

$$
\lim _{t \rightarrow\left(\inf I_{1}\right)^{+}} \gamma_{1}(t)=z, \quad \lim _{t \rightarrow\left(\sup I_{N}\right)^{-}} \gamma_{N}(t)=x,
$$

and which realize the Agmon distance between $x$ and $z$. One has to deal with the two following cases:

1. either $\forall k \in\{1, \ldots, N\}, \forall t \in I_{k}, \gamma_{k}(t) \in V_{\varepsilon}$,

2. or $\exists k \in\{1, \ldots, N\}$ and $\exists t \in I_{k}, \gamma_{k}(t) \in \bar{\Omega} \backslash V_{\varepsilon}$.

In the first case, since $\Phi$ is defined on $V_{\varepsilon}$, it holds

$$
\Phi(x)-f(z)=\Phi(x)-\Phi(z)=\sum_{j=1}^{N} \int_{I_{j}} \frac{d}{d t} \Phi \circ \gamma_{j}(t) d t .
$$

Using Lemma 34 and the fact that $|\nabla \Phi|=g$ on $\Omega \cap V_{\partial \Omega}$ and $\left|\nabla_{T} \Phi\right|=g$ on $\partial \Omega$, it holds, for all $j \in\{1, \ldots, N\}$,

$$
\int_{I_{j}} \frac{d}{d t} \Phi \circ \gamma_{j}(t) d t \leq L\left(\gamma_{j}, I_{j}\right)
$$

and thus

$$
\Phi(x)-f(z) \leq \sum_{j=1}^{N} L\left(\gamma_{j}, I_{j}\right)=d_{a}(x, z) .
$$


Let us now consider the second case. Let us introduce $k_{1} \in\{1, \ldots, N\}$ and $t_{1} \in I_{k_{1}}$ such that for all $t<t_{1}, \gamma_{k_{1}}(t) \in V_{\varepsilon}$, for all $k \in\left\{1, \ldots, k_{1}-1\right\}$, for all $t \in I_{k}, \gamma_{k}(t) \in V_{\varepsilon}$ and such that there exists $\beta>0$ such that for all $t \in\left(t_{1}, t_{1}+\beta\right], \gamma_{k_{1}}(t) \notin V_{\varepsilon}$. The couple $\left(k_{1}, t_{1}\right)$ thus represents the "first time" the curves $\gamma_{1}, \ldots, \gamma_{N}$ leave $V_{\varepsilon}$. Likewise, let us introduce $k_{2} \in\{1, \ldots, N\}$ and $t_{2} \in I_{k_{2}}$ such that for all $t>t_{2}, \gamma_{k_{2}}(t) \in V_{\varepsilon}$, for all $k \in\left\{k_{2}+1, \ldots, N\right\}$, for all $t \in I_{k}, \gamma_{k}(t) \in V_{\varepsilon}$ and such that there exists $\beta>0$ such that for all $t \in\left[t_{2}-\beta, t_{2}\right), \gamma_{k_{2}}(t) \notin V_{\varepsilon}$. The couple $\left(k_{2}, t_{2}\right)$ thus represents the "last time" the curves $\gamma_{1}, \ldots, \gamma_{N}$ leave $\bar{\Omega} \backslash V_{\varepsilon}$. From Step 1 , there is no critical point of $f$ on $\partial V_{\varepsilon} \cap \Omega=\left\{y \in \Omega, f_{-}(y)=\varepsilon\right\}$. Therefore, by construction of the curves $\left(\gamma_{k}\right)_{k=1, \ldots, N}$, the times $t_{1}$ and $t_{2}$ are finite and belong respectively to int $I_{k_{1}}$ and $\operatorname{int} I_{k_{2}}$. One has by continuity of $\gamma_{k_{1}}$ and $\gamma_{k_{2}}, f_{-}\left(\gamma_{k_{1}}\left(t_{1}\right)\right)=f_{-}\left(\gamma_{k_{2}}\left(t_{2}\right)\right)=\varepsilon$. Since $\Phi$ is defined on $V_{\varepsilon}$, using again Lemma 34 and the fact that $|\nabla \Phi|=g$ on $\Omega$ and $\left|\nabla_{T} \Phi\right|=g$, one has

$$
\left|\Phi\left(\gamma_{k_{1}}\left(t_{1}\right)\right)-\Phi(z)\right| \leq \sum_{j=1}^{k_{1}-1} L\left(\gamma_{j}, I_{j}\right)+L\left(\gamma_{k_{1}},\left(\inf I_{k_{1}}, t_{1}\right)\right) .
$$

In addition, using Lemma 63 .

$$
\sum_{j=1}^{k_{1}-1} L\left(\gamma_{j}, I_{j}\right)+L\left(\gamma_{k_{1}},\left(\inf I_{k_{1}}, t_{1}\right)\right)=d_{a}\left(z, \gamma_{k_{1}}\left(t_{1}\right)\right) .
$$

Thus $\left|\Phi\left(\gamma_{k_{1}}\left(t_{1}\right)\right)-\Phi(z)\right| \leq d_{a}\left(z, \gamma_{k_{1}}\left(t_{1}\right)\right)$. By similar arguments, one obtains $\mid \Phi(x)-$ $\Phi\left(\gamma_{k_{2}}\left(t_{2}\right)\right) \mid \leq d_{a}\left(\gamma_{k_{2}}\left(t_{2}\right), x\right)$. Thanks to the definition (108) of $f_{-}$and using the fact that $f_{-}\left(\gamma_{k_{1}}\left(t_{1}\right)\right)=f_{-}\left(\gamma_{k_{2}}\left(t_{2}\right)\right)=\varepsilon$, one has $\left|f\left(\gamma_{k_{2}}\left(t_{2}\right)\right)-f\left(\gamma_{k_{1}}\left(t_{1}\right)\right)\right|=\mid \Phi\left(\gamma_{k_{2}}\left(t_{2}\right)\right)-$ $\Phi\left(\gamma_{k_{1}}\left(t_{1}\right)\right) \mid$. In addition, using (78) one obtains $d_{a}\left(\gamma_{k_{1}}\left(t_{1}\right), \gamma_{k_{2}}\left(t_{2}\right)\right) \geq \mid f\left(\gamma_{k_{2}}\left(t_{2}\right)\right)-$ $f\left(\gamma_{k_{1}}\left(t_{1}\right)\right)|=| \Phi\left(\gamma_{k_{2}}\left(t_{2}\right)\right)-\Phi\left(\gamma_{k_{1}}\left(t_{1}\right)\right) \mid$. Using Lemma 63 and gathering these three last inequalities, one gets

$$
\begin{aligned}
d_{a}(x, z) & =d_{a}\left(z, \gamma_{k_{1}}\left(t_{1}\right)\right)+d_{a}\left(\gamma_{k_{1}}\left(t_{1}\right), \gamma_{k_{2}}\left(t_{2}\right)\right)+d_{a}\left(\gamma_{k_{2}}\left(t_{2}\right), x\right) \\
& \geq\left|\Phi(z)-\Phi\left(\gamma_{k_{1}}\left(t_{1}\right)\right)\right|+\left|\Phi\left(\gamma_{k_{2}}\left(t_{2}\right)\right)-\Phi\left(\gamma_{k_{1}}\left(t_{1}\right)\right)\right|+\left|\Phi(x)-\Phi\left(\gamma_{k_{2}}\left(t_{2}\right)\right)\right| \\
& \geq|\Phi(z)-\Phi(x)| \geq \Phi(x)-\Phi(z)=\Phi(x)-f(z) .
\end{aligned}
$$

Step 3. Let us now show that for all $x \in V_{\Gamma_{z}}, d_{a}(x, z) \leq \Phi(x)-f(z)$.

The proof of this inequality is very similar to the second step in the proof of Proposition 55. For $x \in V_{\Gamma_{z}}$, let $\gamma$ be defined by (96)-(97) (where $\Phi$ is defined by (107)), with $\gamma(0)=x$. The function $\gamma$ is with values in $V_{\Gamma_{z}}$ since $\partial_{n} \Phi>0$ on $\partial V_{\Gamma_{z}} \cap \Omega$ and $\partial V_{\Gamma_{z}} \cap \partial \Omega \subset B_{z}$. Thus $\gamma$ is defined on $\mathbb{R}_{+}$. Thanks to the definition 96 of the vector field $X$, if there exists a time $t_{\partial \Omega}$ such that $\gamma\left(t_{\partial \Omega}\right)$ is in $\partial \Omega$, then, for all $t \geq t_{\partial \Omega}$, $\gamma(t) \in \partial \Omega$. The function $t \in \mathbb{R}_{+} \mapsto \gamma(t)$ is continuous, piecewise $C^{\infty}$ and satisfies

$$
\lim _{t \rightarrow+\infty} \gamma(t)=z
$$

Then, as in the second step of the proof of Proposition 55, one has

$$
\Phi(x)-f(z)=L(\gamma,(0, \infty)) .
$$

Using Lemma 43, one obtains that $d_{a}(x, z) \leq L(\gamma,(0, \infty))=\Phi(x)-f(z)$. This proves the inequality: for all $x \in V_{\Gamma_{z}}, d_{a}(x, z) \leq \Phi(x)-f(z)$. This concludes the proof of Proposition 71 . 
The following corollary is similar to Corollary 64 in the sense that is deals with the case of equality between the Agmon distance and the function $\Phi$ introduced in Proposition 67. Corollary 72 will be needed in the proof of Proposition 91 .

Corollary 72. Let us assume that [H1] and [H3] hold. Let $\Phi$ be the function introduced in Proposition 67 and, let $f_{-}$and $V_{\partial \Omega}$ be respectively the function and the neighborhood of $\partial \Omega$ given by Proposition 70. Let $V_{\alpha}$ be defined by (109), the parameter $\alpha>0$ is chosen such that:

(i) $V_{\alpha} \subset V_{\partial \Omega}$,

(ii) there is no critical point of $f$ on $\partial V_{\alpha} \cap \Omega=\left\{w \in \Omega, f_{-}(w)=\alpha\right\}$,

(iii) $\partial_{n} f>0$ on $\partial V_{\alpha} \cap \Omega$,

(iv) $\partial_{n} f^{-}<0$ on $\partial V_{\alpha} \cap \Omega$, and

(v) $|\nabla \Phi| \neq 0$ in $V_{\alpha}$.

Notice that it is possible to choose such an $\alpha>0$ since $\partial_{n} f^{-}=\partial_{n} \Phi=-\partial_{n} f<0$ on $\partial \Omega=V_{0}$. Let $x, y \in V_{\alpha}$ and denote by $\left(\left(\gamma_{1}, I_{1}\right), \ldots,\left(\gamma_{N}, I_{N}\right)\right)$ the curves given by Theorem 3 ordered such that $\lim _{t \rightarrow\left(\inf I_{1}\right)^{+}} \gamma_{1}(t)=x, \lim _{t \rightarrow\left(\sup I_{N}\right)^{-}} \gamma_{N}(t)=y$ and which realize the Agmon distance between $x$ and $y$. Let us assume that

$$
\Phi(x)-\Phi(y)=d_{a}(x, y)
$$

Then, for all $i \in\{1, \ldots, N\}, \operatorname{Im} \gamma_{i} \subset V_{\alpha}$ and there exist measurable functions $\lambda_{i}: I_{i} \rightarrow$ $\mathbb{R}_{+}$such that for almost every $t$ in $\left\{t \in I_{i}, \gamma_{i}(t) \in \Omega\right\}$, one has $\gamma_{i}^{\prime}(t)=-\lambda_{i}(t) \nabla \Phi\left(\gamma_{i}(t)\right)$, and such that for almost every $t$ in int $\left\{t \in I_{i}, \gamma_{i}(t) \in \partial \Omega\right\}$, one has $\gamma_{i}^{\prime}(t)=-\lambda_{i}(t) \nabla_{T} \Phi\left(\gamma_{i}(t)\right)$. Moreover, if $I_{i}$ is not bounded (namely $I_{i}=(-\infty, 0]$ or $\left.I_{i}=[0,+\infty)\right), \lambda_{i}(t)=1$ for almost every $t \in I_{i}$, and if $I_{i}=[0,1], \lambda_{i} \in L^{\infty}\left([0,1], \mathbb{R}_{+}\right)$.

According to Definition 65, the set of curves $\left\{\gamma_{1}, \ldots, \gamma_{N}\right\}$ introduced in Corollary 72 is a generalized integral curve of the vector field

$$
\left\{\begin{array}{l}
-\nabla \Phi \text { in } V_{\alpha} \cap \Omega, \\
-\nabla_{T} \Phi \text { on } \partial \Omega .
\end{array}\right.
$$

Proof. The proof of this statement is similar to the proof of Corollary 64. Let us first prove that for all $i \in\{1, \ldots, N\}, \operatorname{Im} \gamma_{i} \subset V_{\alpha}$. If it is not the case, then there exist $k \in\{1, \ldots, N\}$ and $t \in I_{k}$ such that $\gamma_{k}(t) \in \bar{\Omega} \backslash V_{\alpha}$. Let the couples $\left(t_{1}, k_{1}\right)$ and $\left(t_{2}, k_{2}\right)$ be defined as in Step 2 of the proof of Proposition 71. Then, one has (see the second step of the proof of Proposition 71), $d_{a}\left(x, \gamma_{k_{1}}\left(t_{1}\right)\right) \geq \Phi(x)-\Phi\left(\gamma_{k_{1}}\left(t_{1}\right)\right)$, $d_{a}\left(\gamma_{k_{1}}\left(t_{1}\right), \gamma_{k_{2}}\left(t_{2}\right)\right) \geq \Phi\left(\gamma_{k_{1}}\left(t_{1}\right)\right)-\Phi\left(\gamma_{k_{2}}\left(t_{2}\right)\right)$ and $d_{a}\left(\gamma_{k_{2}}\left(t_{2}\right), y\right) \geq \Phi\left(\gamma_{k_{2}}\left(t_{2}\right)\right)-\Phi(y)$. Since one has by assumption and from Lemma 63 .

$$
\Phi(x)-\Phi(y)=d_{a}(x, y)=d_{a}\left(x, \gamma_{k_{1}}\left(t_{1}\right)\right)+d_{a}\left(\gamma_{k_{1}}\left(t_{1}\right), \gamma_{k_{2}}\left(t_{2}\right)\right)+d_{a}\left(\gamma_{k_{2}}\left(t_{2}\right), y\right),
$$

all the previous inequalities are equalities and in particular, it holds:

$$
d_{a}\left(\gamma_{k_{1}}\left(t_{1}\right), \gamma_{k_{2}}\left(t_{2}\right)\right)=\Phi\left(\gamma_{k_{1}}\left(t_{1}\right)\right)-\Phi\left(\gamma_{k_{2}}\left(t_{2}\right)\right)=f\left(\gamma_{k_{2}}\left(t_{2}\right)\right)-f\left(\gamma_{k_{1}}\left(t_{1}\right)\right) \geq 0 .
$$


Using Corollary 64, this implies that when restricting $\gamma_{k_{1}}$ to $I_{k_{1}} \cap\left[t_{1}, \infty\right)$ and $\gamma_{k_{2}}$ to $I_{k_{2}} \cap\left(-\infty, t_{2}\right]$ the set of curves $\left\{\gamma_{k_{1}}, \ldots, \gamma_{k_{2}}\right\}$ is a generalized integral curve of

$$
\left\{\begin{array}{c}
\nabla f \text { in } \Omega, \\
\nabla_{T} f \text { on } \partial \Omega
\end{array}\right.
$$

see Definition 65. Let $D=\Omega \backslash V_{\alpha}\left(\partial D=\Omega \cap V_{\alpha}=\left\{w \in \Omega, f_{-}(w)=\alpha\right\}\right.$ is $C^{\infty}$ since $f^{-}$is $C^{\infty}$ and $\partial_{n} f^{-}<0$ on $\partial V_{\alpha} \cap \Omega=\partial D$ which implies that there is no critical point of $f_{-}$on $\partial D$ ). Then, from Corollary 64 and by definition of $\left(t_{1}, k_{1}\right)$ (see the second step of the proof of Proposition 71), there exists $\varepsilon>0$ and a measurable function $\lambda$ : $\left[t_{1}, t_{1}+\varepsilon\right] \rightarrow \mathbb{R}^{+}$such that for all $t \in\left[t_{1}, t_{1}+\varepsilon\right]$ :

$$
\gamma_{k_{1}}^{\prime}(t)=\lambda(t) \nabla f\left(\gamma_{k_{1}}(t)\right)
$$

and for all $t \in\left(t_{1}, t_{1}+\varepsilon\right]$ :

$$
\gamma_{k_{1}}(t) \in D
$$

As in Step 2 of the proof of Proposition 66, let us introduce a smooth tangential and normal system of coordinates around $\gamma_{k_{1}}\left(t_{1}\right) \in \partial D$ in $\bar{D}$, denoted by $\phi(x)=\left(x_{T}, x_{N}\right)$. The function $\phi$ is defined from a neighborhood of $\gamma_{k_{1}}\left(t_{1}\right)$ in $\bar{D}$ to $\mathbb{R}^{d}$. Moreover, one has $x_{N} \geq 0$ and $x_{N}(x)=0$ if and only if $x \in \partial D$. We may assume that the neighborhood $U_{\beta} \subset \bar{D}$ on which $\phi$ is defined is such that $\phi\left(U_{\beta}\right)=U \times[0, \beta]$ for $\beta>0$ and $U \subset \mathbb{R}^{d-1}$. Since $\partial_{n} f>0$ on $\partial D, \beta>0$ can be chosen small enough such that $\nabla f(x) \cdot n(x)>0$ for all $x \in U_{\beta}$ where $n(x)=-\frac{\nabla x_{N}(x)}{\left|\nabla x_{N}(x)\right|}$. Indeed, for $x \in \partial D, n(x)$ is nothing but the unit outward normal to $\partial D$. Now, by continuity of the curve $\gamma_{k_{1}}$, there exists $\mu>0$ such that for all $t \in\left(t_{1}, t_{1}+\mu\right], \gamma_{k_{1}}(t) \in U_{\beta}$. The same considerations as in Step 2 of the proof of Proposition 66 can then be used to show that:

$$
x_{N}(\gamma(t)) \leq 0,
$$

for all $t \in\left[t_{1}, t_{1}+\mu\right]$ and thus $\gamma_{k_{1}}(t) \notin D$ for all $t \in\left[t_{1}, t_{1}+\mu\right]$. This contradicts 110p. Thus, for all $i \in\{1, \ldots, N\}, \operatorname{Im} \gamma_{i} \subset V_{\alpha}$.

Then, the announced result follows by the same arguments as those used in the proof of Corollary 64 with $f$ replaced by $\Phi$ together with the fact that $\Phi$ satisfies (107) on $V_{\alpha}$ and for all $i \in\{1, \ldots, N\}, \operatorname{Im} \gamma_{i} \subset V_{\alpha}$.

\section{Construction of the quasi-modes and proof of Theorem 1}

The aim of this section is to build the quasi-modes $\tilde{u}$ and $\left(\tilde{\phi}_{i}\right)_{i=1, \ldots n}$ satisfying the conditions stated in Section 2.2.2. Let us recall that $\operatorname{span}(\tilde{u})\left(\operatorname{resp} . \operatorname{span}\left(\tilde{\phi}_{i}, i=1, \ldots, n\right)\right)$ is intended to be a good approximation (in the sense made precise in items 1 and 2 in Proposition 25 of $\operatorname{Ran}\left(\pi_{[0, \sqrt{h})}\left(L_{f, h}^{D,(0)}(\Omega)\right)\right)$ (resp. $\left.\operatorname{Ran}\left(\pi_{\left[0, h^{\frac{3}{2}}\right)}\left(\Delta_{f, h}^{D,(1)}(\Omega)\right)\right)\right)$.

As recalled in Proposition 24, it is known that the dimension of $\operatorname{Ran}\left(\pi_{\left[0, h^{\frac{3}{2}}\right)}\left(\Delta_{f, h}^{D,(1)}(\Omega)\right)\right)$ is equal to the number of generalized critical points of index 1 (see [39, Section 3]) which are in our setting, thanks to assumptions [H1], [H2] and [H3], the local minima $\left(z_{i}\right)_{i=1, \ldots, n}$ of $\left.f\right|_{\partial \Omega}$. In addition, it is known that 
the 1-forms in $\operatorname{Ran}\left(\pi_{\left[0, h^{\frac{3}{2}}\right)}\left(\Delta_{f, h}^{D,(1)}(\Omega)\right)\right)$ are localized in the limit $h \rightarrow 0$ in small neighborhoods of the local minima $\left(z_{i}\right)_{i=1, \ldots, n}$.

For each local minimum $z_{i}$, we construct an associated quasi-mode $\tilde{\phi}_{i}$, using an auxiliary Witten Laplacian on 1-forms with mixed tangential-normal boundary conditions. This Witten Laplacian is defined on a domain $\dot{\Omega}_{i} \subset \Omega$ with suitable boundary conditions, so that its only small eigenvalue (namely in the interval $\left[0, h^{\frac{3}{2}}\right)$ ) is 0 , thanks to a complex property (see $[39,54)$ ). The associated eigenform is localized near $z_{i}$, which can be proven thanks to Agmon estimates. Moreover, a precise estimate of this eigenform can be obtained thanks to a WKB expansion. The quasi-mode $\tilde{\phi}_{i}$ is then this eigenform multiplied by a suitable cut-off function.

This section is organized as follows. In Section 4.1, we define a Witten Laplacian with mixed boundary conditions on a open domain $\dot{\Omega}_{i} \subset \Omega$ associated to each $z_{i}$, $i \in\{1, \ldots, n\}$, and we study its spectrum. Section 4.2 is dedicated to the construction of the quasi-modes $\left(\left(\tilde{\phi}_{i}\right)_{i=1, \ldots, n}, \tilde{u}\right)$. In Section 4.3 , we prove Agmon estimates on the eigenform associated with the smallest eigenvalue of the Witten Laplacian with mixed boundary conditions on $\dot{\Omega}_{i}$ and in Section 4.4 we compare this eigenform with a WKB approximation. We finally use this construction and these estimates to prove Theorem 1 in Section 4.5,

\subsection{Geometric setting and definition of the Witten Laplacians with mixed boundary conditions}

This section is organized as follows. In Section 4.1.1, we discuss some general results on traces of differential forms and we introduce the Witten Laplacians with mixed tangential Dirichlet and normal Dirichlet boundary conditions on manifolds with boundary. In Section 4.1.2, the domain $\dot{\Omega}_{i} \subset \Omega$ associated with each $z_{i}, i \in\{1, \ldots, n\}$, is defined. Finally, Section 4.1 .3 is dedicated to the study of the spectrum of the Witten Laplacian with mixed tangential Dirichlet boundary conditions and normal Dirichlet boundary conditions on $\dot{\Omega}_{i}$.

\subsubsection{Trace estimates for differential forms and Witten Laplacians on Lip- schitz domain with mixed boundary conditions}

In this section, we first discuss some general results on traces of differential forms. This is crucial to then build the Witten Laplacians with mixed boundary conditions. In the following, $\dot{\Omega}$ refers to any submanifold $\dot{\Omega}$ of $\Omega$ with Lipschitz boundary. We will call such a submanifold a Lipschitz domain.

We first recall that for any Lipschitz domain $\dot{\Omega}$, the trace application

$$
\left\{\begin{aligned}
\Lambda^{p} H^{1}(\dot{\Omega}) & \rightarrow \Lambda^{p} H^{\frac{1}{2}}(\partial \dot{\Omega}) \\
G & \left.\mapsto G\right|_{\partial \dot{\Omega}}
\end{aligned}\right.
$$

is a linear continuous and surjective application. We would like to present extensions of this result to less regular forms.

\section{Weak definition of traces}

For a Lipschitz domain $\dot{\Omega}$, let us introduce the functional spaces

$$
\Lambda^{p} H_{d}(\dot{\Omega}):=\left\{u \in \Lambda^{p} L^{2}(\dot{\Omega}), d u \in \Lambda^{p+1} L^{2}(\dot{\Omega})\right\}
$$


and

$$
\Lambda^{p} H_{d^{*}}(\dot{\Omega}):=\left\{u \in \Lambda^{p} L^{2}(\dot{\Omega}), d^{*} u \in \Lambda^{p-1} L^{2}(\dot{\Omega})\right\}
$$

equipped with their natural graph norms. One recalls that for a differential form $f$ in $L^{2}(\partial \dot{\Omega})$, the tangential and normal components are defined as follows:

$$
f=\mathbf{t} f+\mathbf{n} f \quad \text { with } \quad \mathbf{t} f=\mathbf{i}_{n}\left(n^{b} \wedge f\right) \quad \text { and } \quad \mathbf{n} f=n^{b} \wedge\left(\mathbf{i}_{n} f\right),
$$

where the superscript $b$ stands for the usual musical isomorphism: $n^{b}$ is the 1-form associated with the outgoing unit normal vector $n$. Moreover,

$$
\|f\|_{L^{2}(\partial \dot{\Omega})}^{2}=\|\mathbf{t} f\|_{L^{2}(\partial \dot{\Omega})}^{2}+\|\mathbf{n} f\|_{L^{2}(\partial \dot{\Omega})}^{2}=\left\|n^{b} \wedge f\right\|_{L^{2}(\partial \dot{\Omega})}^{2}+\left\|\mathbf{i}_{n} f\right\|_{L^{2}(\partial \dot{\Omega})}^{2} .
$$

The Green formula for differential forms $(u, v) \in \Lambda^{p} H^{1}(\dot{\Omega}) \times \Lambda^{p+1} H^{1}(\dot{\Omega})$ writes

$$
\begin{aligned}
\langle d u, v\rangle_{L^{2}(\dot{\Omega})}-\left\langle u, d^{*} v\right\rangle_{L^{2}(\dot{\Omega})} & =\int_{\partial \dot{\Omega}}\left\langle n^{b} \wedge u, v\right\rangle_{T_{\sigma}^{*} \dot{\Omega}} d \sigma=\int_{\partial \dot{\Omega}}\left\langle n^{b} \wedge u, \mathbf{n} v\right\rangle_{T_{\sigma}^{*} \dot{\Omega}} d \sigma \\
& =\int_{\partial \dot{\Omega}}\left\langle u, \mathbf{i}_{n} v\right\rangle_{T_{\sigma}^{*} \dot{\Omega}} d \sigma=\int_{\partial \dot{\Omega}}\left\langle\mathbf{t} u, \mathbf{i}_{n} v\right\rangle_{T_{\sigma}^{*} \dot{\Omega}} d \sigma,
\end{aligned}
$$

where we used the standard relation $\left(n^{b} \wedge\right)^{*}=\mathbf{i}_{n}$.

Using this Green formula, the tangential (resp. normal) traces can be defined for forms in $\Lambda H_{d}(\dot{\Omega})\left(\operatorname{resp} . \Lambda H_{d^{*}}(\dot{\Omega})\right)$ by duality. Indeed, for any $u \in \Lambda^{p} H_{d}(\dot{\Omega}), n^{b} \wedge u \in$ $\Lambda^{p+1} H^{-\frac{1}{2}}(\partial \dot{\Omega})$ is defined by

$$
\forall g \in \Lambda^{p+1} H^{\frac{1}{2}}(\partial \dot{\Omega}),\left\langle n^{b} \wedge u, g\right\rangle_{H^{-\frac{1}{2}}(\partial \dot{\Omega}), H^{\frac{1}{2}}(\partial \dot{\Omega})}=\langle d u, G\rangle_{L^{2}(\dot{\Omega})}-\left\langle u, d^{*} G\right\rangle_{L^{2}(\dot{\Omega})},
$$

where $G$ is any form in $\Lambda^{p+1} H^{1}(\dot{\Omega})$ whose trace in $\Lambda^{p+1} H^{\frac{1}{2}}(\partial \dot{\Omega})$ is $g$. This definition is independent of the chosen extension $G$ (this indeed follows from the Green formula (114) together with the density of $\Lambda^{p} \mathcal{C}^{\infty}(\bar{\Omega})$ in $\Lambda^{p} H_{d}(\dot{\Omega})$, see for example 46 , Proposition 3.1]). Similarly, for any $u \in \Lambda^{p} H_{d^{*}}(\dot{\Omega}), \mathbf{i}_{n} u \in \Lambda^{p-1} H^{-\frac{1}{2}}(\partial \dot{\Omega})$ is defined by

$$
\forall g \in \Lambda^{p-1} H^{\frac{1}{2}}(\partial \dot{\Omega}),\left\langle\dot{i}_{n} u, g\right\rangle_{H^{-\frac{1}{2}}(\partial \dot{\Omega}), H^{\frac{1}{2}}(\partial \dot{\Omega})}=\langle u, d G\rangle_{L^{2}(\dot{\Omega})}-\left\langle d^{*} u, G\right\rangle_{L^{2}(\dot{\Omega})},
$$

where $G$ is any extension of $g$ in $\Lambda^{p-1} H^{1}(\dot{\Omega})$.

Let $\Gamma$ be any subset of $\partial \dot{\Omega}$. For $u \in \Lambda^{p} H_{d}(\dot{\Omega})$, we will write $\left.\mathbf{t} u\right|_{\Gamma}=0$ if $\left.n^{\mathrm{b}} \wedge u\right|_{\Gamma}=0$. If $u \in \Lambda^{p} H_{d}(\dot{\Omega})$ and $\left.n^{b} \wedge u\right|_{\Gamma} \in \Lambda^{p+1} L^{2}(\Gamma)$, the tangential trace on $\Gamma$ is defined by

$$
\left.\mathbf{t} u\right|_{\Gamma}:=\mathbf{i}_{n}\left(n^{b} \wedge u\right) \in \Lambda^{p} L^{2}(\Gamma), \text { so that }\|\mathbf{t} u\|_{L^{2}(\Gamma)}=\left\|n^{b} \wedge u\right\|_{L^{2}(\Gamma)} .
$$

Similarly, for $u \in \Lambda^{p} H_{d^{*}}(\dot{\Omega})$, we will write $\left.\mathbf{n} u\right|_{\Gamma}=0$ if $\left.\mathbf{i}_{n} u\right|_{\Gamma}=0$. If $u \in \Lambda^{p} H_{d^{*}}(\dot{\Omega})$ and $\left.\mathbf{i}_{n} u\right|_{\Gamma} \in \Lambda^{p-1} L^{2}(\Gamma)$, the normal trace on $\Gamma$ is defined by

$$
\left.\mathbf{n} u\right|_{\Gamma}:=n^{b} \wedge\left(\mathbf{i}_{n} u\right) \in \Lambda^{p} L^{2}(\Gamma) \text {, so that }\|\mathbf{n} u\|_{L^{2}(\Gamma)}=\left\|\mathbf{i}_{n} u\right\|_{L^{2}(\Gamma)} .
$$

Lastly, if $u \in \Lambda^{p} H_{d}(\dot{\Omega}) \cap \Lambda^{p} H_{d^{*}}(\dot{\Omega})$ is such that $\left.n^{b} \wedge u\right|_{\Gamma} \in \Lambda^{p+1} L^{2}(\Gamma)$ and $\mathbf{i}_{n} u \in$ $\Lambda^{p-1} L^{2}(\Gamma)$ then $u$ admits a trace $\left.u\right|_{\Gamma}$ in $L^{2}(\Gamma)$ defined by

$$
\left.u\right|_{\Gamma}:=\left.\mathbf{t} u\right|_{\Gamma}+\left.\mathbf{n} u\right|_{\Gamma}
$$

This definition is compatible with 113) and such a differential form satisfies

$$
\left\|\left.u\right|_{\Gamma}\right\|_{L^{2}(\Gamma)}^{2}=\left\|\left.\mathbf{t} u\right|_{\Gamma}\right\|_{L^{2}(\Gamma)}^{2}+\left\|\left.\mathbf{n} u\right|_{\Gamma}\right\|_{L^{2}(\Gamma)}^{2}=\left\|n^{b} \wedge u\right\|_{L^{2}(\Gamma)}^{2}+\left\|\mathbf{i}_{n} u\right\|_{L^{2}(\Gamma)}^{2} .
$$


All the above definitions coincide moreover with the usual ones when $u$ belongs to $\Lambda^{\ell} H^{1}(\dot{\Omega})$.

Let us finally note for further references that if traces are in $L^{2}(\partial \dot{\Omega})$, a direct consequence of the Green formula (114) is the following: for every $u, v \in \Lambda L^{2}(\dot{\Omega})$ such that $d u, d^{*} u, d^{*} d u, d d^{*} u, d v, d^{*} v \in \Lambda L^{2}(\dot{\Omega})$ and $n^{b} \wedge d_{f, h}^{*} u, \mathbf{i}_{n} d_{f, h} u, n^{b} \wedge v, \mathbf{i}_{n} v \in \Lambda L^{2}(\partial \dot{\Omega})$,

$$
\begin{aligned}
& \left\langle\left(d_{f, h} d_{f, h}^{*}+d_{f, h}^{*} d_{f, h}\right) u, v\right\rangle_{L^{2}(\dot{\Omega})}=\left\langle d_{f, h} u, d_{f, h} v\right\rangle_{L^{2}(\dot{\Omega})}+\left\langle d_{f, h}^{*} u, d_{f, h}^{*} v\right\rangle_{L^{2}(\dot{\Omega})} \\
& \quad+h \int_{\partial \dot{\Omega}}\left\langle n^{b} \wedge d_{f, h}^{*} u, n^{b} \wedge\left(\mathbf{i}_{n} v\right)\right\rangle_{T_{\sigma}^{*} \dot{\Omega}} d \sigma-h \int_{\partial \dot{\Omega}}\left\langle n^{b} \wedge v, n^{b} \wedge\left(\mathbf{i}_{n} d_{f, h} u\right)\right\rangle_{T_{\sigma}^{*} \dot{\Omega}} d \sigma .
\end{aligned}
$$

The Gaffney's inequality

The following extension of Gaffney's inequality (see [74]) will be useful in the sequel (we refer to Section 2.1.1 for the definitions of the Hilbert space $\Lambda^{p} H_{T}^{1}(\dot{\Omega})$ and $\left.\Lambda^{p} H_{N}^{1}(\dot{\Omega})\right)$. Notice that in the following result we use that $\dot{\Omega}$ is smooth (there are actually counterexamples for Lipschitz domains, see for example 65]).

Lemma 73. Let $\dot{\Omega}$ be a smooth domain. The equality

$$
\left\{u \in \Lambda^{p} L^{2}(\dot{\Omega}) \text { s.t. } d u, d^{*} u \in L^{2}(\dot{\Omega}) \text { and } \mathbf{t} u=0 \text { on } \partial \dot{\Omega}\right\}=\Lambda^{p} H_{T}^{1}(\dot{\Omega})
$$

holds algebraically and topologically, the functional space in the left-hand side being equipped with the norm associated with the scalar product

$$
Q(u, v):=\langle u, v\rangle_{L^{2}(\dot{\Omega})}+\langle d u, d v\rangle_{L^{2}(\dot{\Omega})}+\left\langle d^{*} u, d^{*} v\right\rangle_{L^{2}(\dot{\Omega})} .
$$

In a similar way, the following equality holds algebraically and topologically:

$$
\left\{u \in \Lambda^{p} L^{2}(\dot{\Omega}) \text { s.t. } d u, d^{*} u \in L^{2}(\dot{\Omega}) \text { and } \mathbf{n} u=0 \text { on } \partial \dot{\Omega}\right\}=\Lambda^{p} H_{N}^{1}(\dot{\Omega}) \text {. }
$$

Notice that in the defintion of the functional spaces above, the equality $\mathbf{t} u=0$ and $\mathbf{n} u=0$ hold in the weak sense defined above (see (117) and (118)). A direct consequence of this lemma is that a differential form in $\Lambda H_{d}(\dot{\Omega}) \cap \Lambda H_{d^{*}}(\dot{\Omega})$ such that $\mathbf{t} u=0$ or $\mathbf{n} u=0$ on $\partial \dot{\Omega}$ admits a trace in $\Lambda L^{2}(\partial \dot{\Omega})$.

Remark 74. The statement of Gaffney's inequality in [74] reads as follows (see indeed Corollary 2.1.6 and Theorem 2.1.7 there):

$$
\exists C>0, \forall u \in \Lambda^{p} H_{T}^{1}(\dot{\Omega}) \cup \Lambda^{p} H_{N}^{1}(\dot{\Omega}),\|u\|_{H^{1}(\dot{\Omega})}^{2} \leq C Q(u, u) .
$$

Since it also holds that, for some $C^{\prime}>0$ and any $u \in \Lambda^{p} H^{1}(\dot{\Omega}), Q(u, u) \leq C^{\prime}\|u\|_{H^{1}(\dot{\Omega})}^{2}$, the scalar products $\langle\cdot, \cdot\rangle_{H^{1}}$ and $Q(\cdot, \cdot)$ are then equivalent on both $\Lambda^{p} H_{T}^{1}(\dot{\Omega})$ and $\Lambda^{p} H_{N}^{1}(\dot{\Omega})$. The above lemma can be seen as a generalization of this result to the spaces $\{u \in$ $\Lambda^{p} L^{2}(\dot{\Omega})$ s.t. $d u, d^{*} u \in L^{2}(\dot{\Omega})$ and $\mathbf{t} u=0$ on $\left.\partial \dot{\Omega}\right\}$ and $\left\{u \in \Lambda^{p} L^{2}(\dot{\Omega})\right.$ s.t. du, $d^{*} u \in$ $L^{2}(\dot{\Omega})$ and $\mathbf{n} u=0$ on $\left.\partial \dot{\Omega}\right\}$.

Proof. We only prove the first equality in Lemma 73 , the second one being similar. Let us define

$$
H:=\left\{u \in \Lambda^{p} L^{2}(\dot{\Omega}) \text { s.t. } d u, d^{*} u \in \Lambda L^{2}(\dot{\Omega}) \quad \text { and } \quad n^{b} \wedge u=0 \text { on } \partial \dot{\Omega}\right\}
$$


which is a Hilbert space once equipped with the scalar product $Q$. From Gaffney's inequality (121), $\Lambda^{p} H_{T}^{1}(\dot{\Omega})$ is a closed subset of $H$ and to conclude, we just have to show that $\left(\Lambda^{p} H_{T}^{1}(\dot{\Omega})\right)^{\perp}=\{0\}$, the orthogonal complement of $H$ being taken with respect to the norm inherited from $Q$. Consider then $u \in H$ such that for any $v \in \Lambda^{p} H_{T}^{1}(\dot{\Omega})$,

$$
0=Q(u, v)=\langle u, v\rangle_{L^{2}(\dot{\Omega})}+\langle d u, d v\rangle_{L^{2}(\dot{\Omega})}+\left\langle d^{*} u, d^{*} v\right\rangle_{L^{2}(\dot{\Omega})} .
$$

The above equality holds in particular for every $v \in D$ where

$$
D=\left\{v \in \Lambda^{p} H^{2}(\dot{\Omega}),\left.\mathbf{t} v\right|_{\partial \dot{\Omega}}=\left.\mathbf{t} d^{*} v\right|_{\partial \dot{\Omega}}=0\right\}
$$

Fix such a $v$. Since $n^{b} \wedge u=0$ on $\partial \dot{\Omega}$, applying (115) to $u$ and $d v \in \Lambda^{p+1} H^{1}(\dot{\Omega})$ then leads to

$$
\langle d u, d v\rangle_{L^{2}(\dot{\Omega})}=\left\langle u, d^{*} d v\right\rangle_{L^{2}(\dot{\Omega})} .
$$

Applying also 116 to $u$ and $d^{*} v \in \Lambda^{p-1} H^{1}(\dot{\Omega})$ gives

$$
\left\langle d^{*} u, d^{*} v\right\rangle_{L^{2}(\dot{\Omega})}=\left\langle u, d d^{*} v\right\rangle_{L^{2}(\dot{\Omega})}-\left\langle\mathbf{i}_{n} u,\left.d^{*} v\right|_{\partial \dot{\Omega}}\right\rangle_{H^{-\frac{1}{2}}(\partial \dot{\Omega}), H^{\frac{1}{2}}(\partial \dot{\Omega})}
$$

Since $\Lambda^{p} \mathcal{C}^{\infty}(\bar{\Omega})$ is densely embedded in both $\Lambda^{p} H_{d}(\dot{\Omega})$ and $\Lambda^{p} H_{d^{*}}(\dot{\Omega})$ (see for example 46. Proposition 3.1]), we have moreover for some sequence $\left(u_{k}\right)_{k \in \mathbb{N}}$ of $\Lambda^{p} \mathcal{C}^{\infty}(\dot{\bar{\Omega}})$ forms:

$$
\begin{aligned}
\left\langle\mathbf{i}_{n} u,\left.d^{*} v\right|_{\partial \dot{\Omega}}\right\rangle_{H^{-\frac{1}{2}}(\partial \dot{\Omega}), H^{\frac{1}{2}}(\partial \dot{\Omega})} & =\lim _{k \rightarrow+\infty} \int_{\partial \dot{\Omega}}\left\langle\mathbf{i}_{n} u_{k}, d^{*} v\right\rangle_{T_{\sigma}^{*} \dot{\Omega}} d \sigma \\
& =\lim _{k \rightarrow+\infty} \int_{\partial \dot{\Omega}}\left\langle\mathbf{i}_{n} u_{k}, n^{b} \wedge\left(\mathbf{i}_{n} d^{*} v\right)\right\rangle_{T_{\sigma}^{*} \dot{\Omega}} d \sigma=0
\end{aligned}
$$

where the second equality is a consequence of $\left.\mathbf{t} d^{*} v\right|_{\partial \dot{\Omega}}=0$. It consequently follows

$$
0=\langle u, v\rangle_{L^{2}(\dot{\Omega})}+\left\langle u, d^{*} d v\right\rangle_{L^{2}(\dot{\Omega})}+\left\langle u, d d^{*} v\right\rangle_{L^{2}(\dot{\Omega})}=\left\langle u,\left(I+\Delta_{H}^{(p)}\right) v\right\rangle_{L^{2}(\dot{\Omega})},
$$

where $\Delta_{H}^{(p)}$ denotes the Hodge Laplacian on $\dot{\Omega}$ with domain $D$ defined by $(122)$. Since the unbounded operator $\left(\Delta_{H}^{(p)}, D\right)$ is selfadjoint and nonnegative on $\Lambda^{p} L^{2}(\dot{\Omega})$, we have in particular $\operatorname{Ran}\left(I+\Delta_{H}^{(p)}\right)=\Lambda^{p} L^{2}(\dot{\Omega})$ and we deduce from 123 that $u=0$, which completes the proof.

The case of mixed normal-tangential Dirichlet boundary conditions

Let $\Gamma_{T}$ and $\Gamma_{N}$ be two disjoint open subsets of $\partial \dot{\Omega}$ such that $\overline{\Gamma_{T}} \cup \overline{\Gamma_{N}}=\partial \dot{\Omega}$. The objective of this section is to consider differential forms such that $\mathbf{t} u=0$ on $\Gamma_{T}$ and $\mathbf{n} u=0$ on $\Gamma_{N}$, and to state results on the existence of a trace in $L^{2}(\partial \dot{\Omega})$ for such differential forms, as well as subelliptic estimates.

In general, a trace in $L^{2}(\partial \dot{\Omega})$ does not exist in such a setting [8, 46]: one needs a geometric assumption, namley that $\Gamma_{T}$ and $\Gamma_{N}$ meet at an angle strictly smaller than $\pi$. This means that the angle between $\Gamma_{T}$ and $\Gamma_{N}$ measured in $\dot{\Omega}$ is smaller than $\pi$. More precisely, see [8, 46], locally around any point $x_{0} \in \overline{\Gamma_{T}} \cap \overline{\Gamma_{N}}$, one requires that there exists a local system of coordinates $\left(x_{1}, x^{\prime \prime}, x_{n}\right) \in \mathbb{R} \times \mathbb{R}^{d-2} \times \mathbb{R}$ on a neighborhood $V_{0}$ of $x_{0}$, and two Lipschitz functions $\tilde{\varphi}: \mathbb{R}^{n-1} \rightarrow \mathbb{R}$ and $\tilde{\psi}: \mathbb{R}^{n-2} \rightarrow \mathbb{R}$ such that 
$\dot{\Omega} \cap V_{0}=\left\{x_{n}>\tilde{\varphi}\left(x_{1}, x^{\prime \prime}\right)\right\}, \Gamma_{T} \cap V_{0}=\left\{x_{n}=\tilde{\varphi}\left(x_{1}, x^{\prime \prime}\right)\right.$ and $\left.x_{1}>\tilde{\psi}\left(x^{\prime \prime}\right)\right\}$ and $\Gamma_{N} \cap V_{0}=$ $\left\{x_{n}=\tilde{\varphi}\left(x_{1}, x^{\prime \prime}\right)\right.$ and $\left.x_{1}<\tilde{\psi}\left(x^{\prime \prime}\right)\right\}$ and

$$
\begin{array}{r}
\partial_{x_{1}} \tilde{\varphi}\left(x_{1}, x^{\prime \prime}\right) \geq \kappa \text { on } x_{1}>\tilde{\psi}\left(x^{\prime \prime}\right) \\
\partial_{x_{1}} \tilde{\varphi}\left(x_{1}, x^{\prime \prime}\right) \leq-\kappa \text { on } x_{1}<\tilde{\psi}\left(x^{\prime \prime}\right)
\end{array}
$$

for some positive $\kappa$. This is equivalent to the existence of a smooth vector field $\theta$ on $\partial \dot{\Omega}$ such that $\langle\theta, n\rangle<0$ on $\Gamma_{T}$ and $\langle\theta, n\rangle>0$ on $\Gamma_{N}$, which is one of the key ingredient of the proofs used in 8,46 .

Let $\Gamma$ be any open Lipschitz subset of $\partial \dot{\Omega}$. According to [46, Proposition 3.1], the space

$$
\left\{u \in \Lambda^{p} \mathcal{C}^{\infty}(\overline{\dot{\Omega}}), u \equiv 0 \text { in a neighborhood of } \partial \dot{\Omega} \backslash \Gamma\right\}
$$

is densely embedded in both

$$
\Lambda^{p} H_{d, \Gamma}(\dot{\Omega}):=\left\{u \in \Lambda^{p} H_{d}(\dot{\Omega}), \operatorname{supp}\left(n^{b} \wedge u\right) \subset \bar{\Gamma}\right\}
$$

and

$$
\Lambda^{p} H_{d^{*}, \Gamma}(\dot{\Omega}):=\left\{u \in \Lambda^{p} H_{d^{*}}(\dot{\Omega}), \operatorname{supp}\left(\mathbf{i}_{n} u\right) \subset \bar{\Gamma}\right\} .
$$

In addition, according to 46 , Theorem 3.4], for $(u, v) \in \Lambda^{p} H_{d}(\dot{\Omega}) \times \Lambda^{p+1} H_{d^{*}}(\dot{\Omega})$ satisfying the trace conditions

$$
\mathbf{i}_{n} v \in \Lambda^{p} L^{2}(\partial \dot{\Omega}), \operatorname{supp} \mathbf{i}_{n} v \subset \Gamma \quad \text { and } \quad n^{b} \wedge u \in \Lambda^{p+1} L^{2}(\Gamma),
$$

or

$$
n^{b} \wedge u \in \Lambda^{p+1} L^{2}(\partial \dot{\Omega}), \operatorname{supp}\left(n^{b} \wedge u\right) \subset \Gamma \quad \text { and } \quad \mathbf{i}_{n} v \in \Lambda^{p} L^{2}(\Gamma),
$$

one has the following Green formula (compare with (114)):

$$
\begin{aligned}
\langle d u, v\rangle_{L^{2}(\dot{\Omega})}-\left\langle u, d^{*} v\right\rangle_{L^{2}(\dot{\Omega})} & =\int_{\Gamma}\left\langle n^{b} \wedge u, n^{b} \wedge\left(\mathbf{i}_{n} v\right)\right\rangle_{T_{\sigma}^{*} \dot{\Omega}} d \sigma \\
& =\int_{\Gamma}\left\langle\mathbf{i}_{n}\left(n^{b} \wedge u\right), \mathbf{i}_{n} v\right\rangle_{T_{\sigma}^{*} \dot{\Omega}} d \sigma .
\end{aligned}
$$

One is now ready to state the following proposition implied by Theorems 1.1 and 1.2 of 46 (see also Theorems 4.1 and 4.2 of [33]).

Proposition 75. Let us assume that $\dot{\Omega}$ is a Lipschitz domain. Let $\Gamma_{T}$ and $\Gamma_{N}$ be two disjoint Lipschitz open subsets of $\partial \dot{\Omega}$ such that $\overline{\Gamma_{T}} \cup \overline{\Gamma_{N}}=\partial \dot{\Omega}$ and such that $\Gamma_{T}$ and $\Gamma_{N}$ meet at an angle strictly smaller than $\pi$. Then, the following results hold:

(i) Let $u$ be a differential form such that

$$
u \in \Lambda^{p} L^{2}(\dot{\Omega}), d u \in L^{2}(\dot{\Omega}), d^{*} u \in L^{2}(\dot{\Omega}),\left.\mathbf{t} u\right|_{\Gamma_{T}}=0 \text { and }\left.\mathbf{n} u\right|_{\Gamma_{N}}=0 .
$$

Then $u$ satisfies

$$
u \in \Lambda^{p} H^{\frac{1}{2}}(\dot{\Omega}) \quad \text { and } \quad \mathbf{i}_{n} u, n^{b} \wedge u \in \Lambda^{p} L^{2}(\partial \dot{\Omega})
$$

as well as the subelliptic estimate:

$$
\|u\|_{H^{\frac{1}{2}(\dot{\Omega})}}+\left\|\left.u\right|_{\partial \dot{\Omega}}\right\|_{L^{2}(\partial \dot{\Omega})} \leq C\left(\|u\|_{L^{2}(\dot{\Omega})}+\|d u\|_{L^{2}(\dot{\Omega})}+\left\|d^{*} u\right\|_{L^{2}(\dot{\Omega})}\right),
$$

where $\left.u\right|_{\partial \dot{\Omega}}$ is defined by 119 . 
(ii) The unbounded operators $d_{T}^{(p)}(\dot{\Omega})$ and $\delta_{N}^{(p)}(\dot{\Omega})$ on $\Lambda^{p} L^{2}(\dot{\Omega})$ defined by

$$
d_{T}^{(p)}(\dot{\Omega})=d_{f, h}^{(p)}
$$

with domain

$$
D\left(d_{T}^{(p)}(\dot{\Omega})\right)=\left\{u \in \Lambda^{p} L^{2}(\dot{\Omega}), d_{f, h} u \in \Lambda^{p+1} L^{2}(\dot{\Omega}),\left.\mathbf{t} u\right|_{\Gamma_{T}}=0\right\}
$$

and

$$
\delta_{N}^{(p)}(\dot{\Omega})=\left(d_{f, h}^{(p)}\right)^{*}
$$

with domain

$$
D\left(\delta_{N}^{(p)}(\dot{\Omega})\right)=\left\{u \in \Lambda^{p} L^{2}(\dot{\Omega}), d_{f, h}^{*} u \in \Lambda^{p-1} L^{2}(\dot{\Omega}),\left.\mathbf{n} u\right|_{\Gamma_{N}}=0\right\},
$$

are closed, densely defined, and adjoint one of each other.

Note that in the point (i) of Proposition 75, $d$ and $d^{*}$ can be replaced by $d_{f, h}$ and $d_{f, h}^{*}$ owing to the relations $d_{f, h}=h d+d f \wedge$ and $d_{f, h}^{*}=h d^{*}+\mathbf{i}_{\nabla f}$. Moreover, the point (ii) is actually proven in [33,46] for $d$ and $d^{*}$ but remains true for $d_{f, h}$ and $d_{f, h}^{*}$ since $(d f \wedge)^{*}=\mathbf{i}_{\nabla f}$ on $L^{2}(\dot{\Omega})$.

The mixed Witten Laplacian $\Delta_{f, h}^{M}(\dot{\Omega})$

We are now in position to define the mixed Witten Laplacian $\Delta_{f, h}^{M}(\dot{\Omega})$ (the upperscript $M$ stands for mixed boundary conditions) with tangential Dirichlet boundary conditions on $\Gamma_{T}$ and normal Dirichlet boundary conditions on $\Gamma_{N}$ (see 33,46 for more results on such operators). The operator $\Delta_{f, h}^{M,(p)}(\dot{\Omega})$ on $L^{2}(\dot{\Omega})$ is defined by

$$
\Delta_{f, h}^{M,(p)}(\dot{\Omega}):=d_{T}^{(p-1)}(\dot{\Omega}) \circ \delta_{N}^{(p)}(\dot{\Omega})+\delta_{N}^{(p+1)}(\dot{\Omega}) \circ d_{T}^{(p)}(\dot{\Omega}),
$$

in the sense of composition of unbounded operators, where $d_{T}$ and $\delta_{N}$ have been introduced in Proposition 75. Notice that for any $u \in \Lambda^{p} H_{d}(\dot{\Omega})$ such that $\left.\mathbf{t} u\right|_{\Gamma_{T}}=0$, one has $d u \in \Lambda^{p+1} H_{d}(\dot{\Omega})$ and $\left.\mathbf{t} d u\right|_{\Gamma_{T}}=0$. The latter is easy to check when $u \in \Lambda^{p} H^{2}(\dot{\Omega})$ and can be proved here using (115) together with the density of $\left\{u \in \Lambda^{p} \mathcal{C}^{\infty}(\bar{\Omega}), u \equiv\right.$ 0 in a neighborhood of $\left.\partial \dot{\Omega} \backslash \overline{\Gamma_{T}}\right\}$ into $\Lambda^{p} H_{d, \partial \dot{\Omega} \backslash \overline{\Gamma_{T}}}(\dot{\Omega})$. Likewise, one has $d_{f, h} d_{f, h}=0$ in the distributional sense and $\left.\mathbf{t} d_{f, h} u\right|_{\Gamma_{T}}=0$ for $u \in \Lambda^{p} H_{d}(\dot{\Omega})$ such that $\left.\mathbf{t} u\right|_{\Gamma_{T}}=0$. This implies in particular

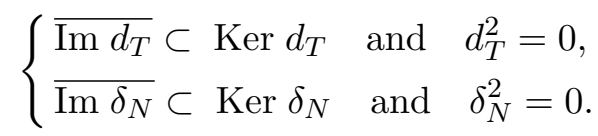

Owing to this last relation and to Proposition 75, a result due to Gaffney (see e.g. the proof of 33 . Propositions 2.3 and 2.4]) states that $\Delta_{f, h}^{M,(p)}(\dot{\Omega})$ is a densely defined nonnegative selfadjoint operator on $L^{2}(\dot{\Omega})$ (with domain defined below in 129 ).

The domain $D\left(\mathcal{Q}_{f, h}^{M,(p)}(\dot{\Omega})\right)$ of the closed quadratic form $\mathcal{Q}_{f, h}^{M,(p)}(\dot{\Omega})$ associated with $\Delta_{f, h}^{M,(p)}(\dot{\Omega})$ is given by

$$
\begin{aligned}
D\left(\mathcal{Q}_{f, h}^{M,(p)}(\dot{\Omega})\right) & =D\left(d_{T}^{(p)}(\dot{\Omega})\right) \cap D\left(\delta_{N}^{(p)}(\dot{\Omega})\right) \\
& =\left\{v \in \Lambda^{p} L^{2}(\dot{\Omega}), d v \in L^{2}(\dot{\Omega}), d^{*} v \in L^{2}(\dot{\Omega}),\left.\mathbf{t} v\right|_{\Gamma_{T}}=0 \text { and }\left.\mathbf{n} v\right|_{\Gamma_{N}}=0\right\}
\end{aligned}
$$


and for any $u, v \in D\left(\mathcal{Q}_{f, h}^{M,(p)}(\dot{\Omega})\right)$,

$$
\mathcal{Q}_{f, h}^{M,(p)}(\dot{\Omega})(u, v)=\left\langle d_{T} u, d_{T} v\right\rangle_{L^{2}}+\left\langle\delta_{N} u, \delta_{N} v\right\rangle_{L^{2}} .
$$

This is proven in [33, Theorem 2.8].

The domain $D\left(\Delta_{f, h}^{M,(p)}(\dot{\Omega})\right)$ is explicitly given by:

$$
\begin{aligned}
& D\left(\Delta_{f, h}^{M,(p)}(\dot{\Omega})\right)=\left\{u \in L^{2}(\dot{\Omega}) \text { s.t. } d_{f, h} u, d_{f, h}^{*} u, d_{f, h}^{*} d_{f, h} u, d_{f, h} d_{f, h}^{*} u \in L^{2}(\dot{\Omega}),\right. \\
& \left.\left.\mathbf{t} u\right|_{\Gamma_{T}}=0,\left.\mathbf{t} d_{f, h}^{*} u\right|_{\Gamma_{T}}=0,\left.\mathbf{n} u\right|_{\Gamma_{N}}=0,\left.\mathbf{n} d_{f, h} u\right|_{\Gamma_{N}}=0\right\} .
\end{aligned}
$$

The traces $\mathbf{t} d_{f, h}^{*} u$ and $\mathbf{n} d_{f, h} u$ are a priori defined in $H^{-\frac{1}{2}}(\partial \dot{\Omega})$ but actually belong to $L^{2}(\partial \dot{\Omega})$. Indeed, we have $\left.\mathbf{n} d_{f, h} u\right|_{\Gamma_{N}}=0$ by definition of $D\left(\Delta_{f, h}^{M,(p)}(\dot{\Omega})\right)$ and $\left.\mathbf{t} d_{f, h} u\right|_{\Gamma_{T}}=$ 0 by $(128)$, so $d_{f, h} u$ is in $D\left(\mathcal{Q}_{f, h}^{M,(p+1)}(\dot{\Omega})\right)$ and therefore has a trace in $L^{2}(\dot{\Omega})$ according to Proposition 75. This argument also holds for $d_{f, h}^{*} u \in D\left(\mathcal{Q}_{f, h}^{M,(p-1)}(\dot{\Omega})\right)$.

We end up this section with the following lemma which will be frequently used in the sequel.

Lemma 76. Let us assume that the assumptions of Proposition 75 are satisfied. Let us moreover assume that $\Gamma_{T}$ is $C^{\infty}$ and that there exist two disjoint $C^{\infty}$ open subsets $\Gamma_{N, 1}$ and $\Gamma_{N, 2}$ of $\partial \dot{\Omega}$ such that

$$
\overline{\Gamma_{N}}=\overline{\Gamma_{N, 1}} \cup \overline{\Gamma_{N, 2}} .
$$

Then, the following formula holds: for any $u \in D\left(\mathcal{Q}_{f, h}^{M,(p)}(\dot{\Omega})\right)$,

$$
\begin{aligned}
\mathcal{Q}_{f, h}^{M,(p)}(\dot{\Omega})(u, u)= & \left\|d_{f, h} u\right\|_{L^{2}(\dot{\Omega})}^{2}+\left\|d_{f, h}^{*} u\right\|_{L^{2}(\dot{\Omega})}^{2} \\
= & h^{2}\|d u\|_{L^{2}(\dot{\Omega})}^{2}+h^{2}\left\|d^{*} u\right\|_{L^{2}(\dot{\Omega})}^{2} \\
& +\||\nabla f| u\|_{L^{2}(\dot{\Omega})}^{2}+h\left\langle\left(\mathcal{L}_{\nabla f}+\mathcal{L}_{\nabla f}^{*}\right) u, u\right\rangle_{L^{2}(\dot{\Omega})} \\
& -h\left(\int_{\Gamma_{T}}-\int_{\Gamma_{N}}\right)\langle u, u\rangle_{T_{\sigma}^{*} \dot{\Omega}} \partial_{n} f d \sigma,
\end{aligned}
$$

where $\mathcal{L}$ stands for the Lie derivative.

Notice that the boundary integral terms are well defined since $\left.u\right|_{\partial \dot{\Omega}} \in L^{2}(\dot{\Omega})$ thanks to point (i) in Proposition 75. From the proof of Lemma 76, it will be clear that (131) actually holds if $\Gamma_{T}$ and $\Gamma_{N}$ are only piecewise smooth. In the following, we will only need the result for $\Gamma_{T}$ smooth and $\Gamma_{N}$ the union of two smooth pieces and this is why we present this result in this setting.

Proof. For $u \in D\left(\mathcal{Q}_{f, h}^{M,(p)}(\dot{\Omega})\right)$, one first gets by straightforward computations,

$$
\begin{aligned}
\left\|d_{f, h} u\right\|_{L^{2}(\dot{\Omega})}^{2}+\left\|d_{f, h}^{*} u\right\|_{L^{2}(\dot{\Omega})}^{2}= & h^{2}\|d u\|_{L^{2}(\dot{\Omega})}^{2}+h^{2}\left\|d^{*} u\right\|_{L^{2}(\dot{\Omega})}^{2}+\|d f \wedge u\|_{L^{2}(\dot{\Omega})}^{2} \\
& +\left\|\mathbf{i}_{\nabla f} u\right\|_{L^{2}(\dot{\Omega})}^{2}+h\langle d f \wedge u, d u\rangle_{L^{2}(\dot{\Omega})}+h\langle d u, d f \wedge u\rangle_{L^{2}(\dot{\Omega})} \\
& +h\left\langle d^{*} u, \mathbf{i}_{\nabla f} u\right\rangle_{L^{2}(\dot{\Omega})}+h\left\langle\mathbf{i}_{\nabla f} u, d^{*} u\right\rangle_{L^{2}(\dot{\Omega})} \\
= & h^{2}\|d u\|_{L^{2}(\dot{\Omega})}^{2}+h^{2}\left\|d^{*} u\right\|_{L^{2}(\dot{\Omega})}^{2}+\||\nabla f| u\|_{L^{2}(\dot{\Omega})}^{2} \\
& +h\left\langle\left(\mathcal{L}_{\nabla f}+\mathcal{L}_{\nabla f}^{*}\right) u, u\right\rangle_{L^{2}(\dot{\Omega})}+h\langle d f \wedge u, d u\rangle_{L^{2}(\dot{\Omega})} \\
& -h\left\langle d^{*}(d f \wedge u), u\right\rangle_{L^{2}(\dot{\Omega})}-h\left\langle d \mathbf{i}_{\nabla f} u, u\right\rangle_{L^{2}(\dot{\Omega})}+h\left\langle\mathbf{i}_{\nabla f} u, d^{*} u\right\rangle_{L^{2}(\dot{\Omega})},
\end{aligned}
$$


where the last equality holds thanks to the relations

$$
(d f \wedge)^{*}=\mathbf{i}_{\nabla f}, \mathcal{L}_{\nabla f}=d \circ \mathbf{i}_{\nabla f}+\mathbf{i}_{\nabla f} \circ d \quad \text { and } \quad \mathbf{i}_{\nabla f}(d f \wedge u)+d f \wedge\left(\mathbf{i}_{\nabla f} u\right)=|\nabla f|^{2} u .
$$

To get the boundary integral terms in 131) one uses (125), which gives here, since $u \in D\left(\mathcal{Q}_{f, h}^{M,(p)}(\dot{\Omega})\right)$ and $d f \wedge u, \mathbf{i}_{\nabla f} u \in \Lambda H_{d}(\dot{\Omega}) \cap \Lambda H_{d^{*}}(\dot{\Omega})$ :

$$
\begin{aligned}
& \langle d f \wedge u, d u\rangle_{L^{2}(\dot{\Omega})}-\left\langle d^{*}(d f \wedge u), u\right\rangle_{L^{2}(\dot{\Omega})}=\int_{\Gamma_{N}}\left\langle n^{b} \wedge u, n^{b} \wedge \mathbf{i}_{n}(d f \wedge u)\right\rangle_{T_{\sigma}^{*} \dot{\Omega}} d \sigma \\
& =\int_{\Gamma_{N, 1}}\left\langle n^{b} \wedge u, n^{b} \wedge \mathbf{i}_{n}(d f \wedge u)\right\rangle_{T_{\sigma}^{*} \dot{\Omega}} d \sigma+\int_{\Gamma_{N, 2}}\left\langle n^{b} \wedge u, n^{b} \wedge \mathbf{i}_{n}(d f \wedge u)\right\rangle_{T_{\sigma}^{*} \dot{\Omega}} d \sigma
\end{aligned}
$$

where the last equality follows from 130 . Likewise, one has:

$$
\left\langle\mathbf{i}_{\nabla f} u, d^{*} u\right\rangle_{L^{2}(\dot{\Omega})}-\left\langle d \mathbf{i}_{\nabla f} u, u\right\rangle_{L^{2}(\dot{\Omega})}=-\int_{\Gamma_{T}}\left\langle n^{b} \wedge \mathbf{i}_{\nabla f} u, n^{b} \wedge \mathbf{i}_{n} u\right\rangle_{T_{\sigma}^{*} \dot{\Omega}} d \sigma
$$

Since $u \in D\left(\mathcal{Q}_{f, h}^{M,(p)}(\dot{\Omega})\right)$, and $\Gamma_{T}, \Gamma_{N, 1}$, and $\Gamma_{N, 2}$ are smooth open subsets of $\partial \dot{\Omega}$, Lemma 73 implies that $u$ is in $\Lambda^{p} H^{1}$ outside $\left(\overline{\Gamma_{T}} \cap \overline{\Gamma_{N}}\right) \cup\left(\overline{\Gamma_{N, 1}} \cap \overline{\Gamma_{N, 2}}\right)$, by a localization argument. Therefore, $u$ admits a boundary trace defined a.e. on $\partial \dot{\Omega}$ and belonging to $L_{\text {loc }}^{2}\left(\partial \dot{\Omega} \backslash\left(\overline{\Gamma_{T}} \cap \overline{\Gamma_{N}}\right) \cup\left(\overline{\Gamma_{N, 1}} \cap \overline{\Gamma_{N, 2}}\right)\right)$. But this trace has to be $\left.u\right|_{\partial \dot{\Omega}}$ as defined by (119) and is hence in $L^{2}(\partial \dot{\Omega})$ owing to item $(i)$ in Proposition 75. Let us now conclude the proof of Lemma 76. Let us consider (133). For $j \in\{1,2\}$ and $\varepsilon>0$, one defines $\Gamma_{N, j}^{\varepsilon}:=\left\{x \in \Gamma_{N, j}, d^{\partial \Omega}\left(x, \partial \Gamma_{N, j}\right)>\varepsilon\right\}$. One then has for $j \in\{1,2\}$ :

$$
\begin{aligned}
\int_{\Gamma_{N, j}}\left\langle n^{b} \wedge u, n^{b} \wedge \mathbf{i}_{n}(d f \wedge u)\right\rangle_{T_{\sigma}^{*} \dot{\Omega}} d \sigma & =\lim _{\varepsilon \rightarrow 0^{+}} \int_{\Gamma_{N, j}^{\varepsilon}}\left\langle n^{b} \wedge u, n^{b} \wedge \mathbf{i}_{n}(d f \wedge u)\right\rangle_{T_{\sigma}^{*} \dot{\Omega}} d \sigma \\
& =\lim _{\varepsilon \rightarrow 0^{+}} \int_{\Gamma_{N, j}^{\varepsilon}}\left\langle u, \mathbf{i}_{n}\left(n^{b} \wedge \mathbf{i}_{n}(d f \wedge u)\right)\right\rangle_{T_{\sigma}^{* \dot{\Omega}}} d \sigma \\
& =\lim _{\varepsilon \rightarrow 0^{+}} \int_{\Gamma_{N, j}^{\varepsilon}}\left\langle u, \mathbf{i}_{n}(d f \wedge u)\right\rangle_{T_{\sigma}^{*} \dot{\Omega}} d \sigma \\
& =\lim _{\varepsilon \rightarrow 0^{+}} \int_{\Gamma_{N, j}^{\varepsilon}}\left(\partial_{n} f\langle u, u\rangle_{T_{\sigma}^{*} \dot{\Omega}}-\left\langle u, d f \wedge \mathbf{i}_{n} u\right\rangle_{T_{\sigma}^{*} \dot{\Omega}}\right) d \sigma \\
& =\int_{\Gamma_{N, j}} \partial_{n} f\langle u, u\rangle_{T_{\sigma}^{*} \dot{\Omega}} d \sigma,
\end{aligned}
$$

where we used the usual trace properties for $H^{1}$ forms on $\Gamma_{N, j}^{\varepsilon}$, the fact that $\mathbf{i}_{n} u=0$ at the second to last line and the Lebesgue dominated convergence theorem at the last line. From 133, one then has:

$$
\int_{\Gamma_{N}}\left\langle n^{b} \wedge u, n^{b} \wedge \mathbf{i}_{n}(d f \wedge u)\right\rangle_{T_{\sigma}^{*} \dot{\Omega}} d \sigma=\int_{\Gamma_{N}} \partial_{n} f\langle u, u\rangle_{T_{\sigma}^{*} \dot{\Omega}} d \sigma .
$$

The fact that $\int_{\Gamma_{T}}\left\langle n^{b} \wedge \mathbf{i}_{\nabla f} u, n^{b} \wedge \mathbf{i}_{n} u\right\rangle_{T_{\sigma}^{*} \dot{\Omega}} d \sigma=\int_{\Gamma_{T}} \partial_{n} f\langle u, u\rangle_{T_{\sigma}^{*} \dot{\Omega}} d \sigma$ is proved similarly. This concludes the proof of Lemma 76 .

\subsubsection{Construction of the domain $\dot{\Omega}_{i}$}

In this section, we assume [H1], [H2] and [H3]. Let us consider $z_{i} \in\left\{z_{1}, \ldots, z_{n}\right\}$ a local minimum of $\left.f\right|_{\partial \Omega}$. The objective of this section is to build the domain $\dot{\Omega}_{i}$ on which 
the Witten Laplacian with mixed tangential-normal Dirichlet boundary conditions will be defined. This auxiliary operator is such that $z_{i}$ remains the only generalized critical point.

Let us recall that $x_{0} \in \Omega$ is the minimum of $f$ on $\bar{\Omega}$. Let $\Omega_{0}$ be a small smooth open neighborhood of $x_{0}$ such that the $\partial_{n} f<0$ on $\Gamma_{0}=\partial \Omega_{0}, n$ being the outward normal derivative to $\Omega \backslash \Omega_{0}$. Let $\Gamma_{1, i}$ denote a subset of $B_{z_{i}}$, as large as we want in $B_{z_{i}}$, and such that $z_{i} \in \Gamma_{1, i}$. The basic idea is to define $\dot{\Omega}=\Omega \backslash \overline{\Omega_{0}}$ and to consider a Witten Laplacian on $\dot{\Omega}$, with tangential Dirichlet boundary conditions on $\Gamma_{0} \cup \Gamma_{1, i}$ and with normal Dirichlet boundary conditions on $\partial \Omega \backslash \overline{\Gamma_{1, i}}$. This would indeed yield an operator on a domain $\dot{\Omega}$ with a single generalized critical point, namely $z_{i}$.

There is however a technical difficulty in this approach, related to the fact that differential forms with mixed normal and tangential Dirichlet boundary conditions are singular at the boundary between the domains where tangential and normal boundary conditions are applied, as explained in Section 4.1.1. With the previous construction, $\Gamma_{1, i}$ and $\partial \Omega \backslash \overline{\Gamma_{1, i}}$ meet at an angle $\pi$. We therefore need to define a domain $\dot{\Omega}_{i}$ stricly included in $\Omega \backslash \overline{\Omega_{0}}$, with boundary $\partial \dot{\Omega}_{i}=\overline{\Gamma_{0}} \cup \overline{\Gamma_{1, i}} \cup \overline{\Gamma_{2, i}}$ where $\Gamma_{0}=\partial \Omega_{0}$ as defined above, $\Gamma_{1, i} \cap \Gamma_{2, i}=\emptyset, \Gamma_{1, i} \subset B_{z_{i}}$ is as large as we want in $B_{z_{i}}$ and $\Gamma_{2, i}$ meets $\Gamma_{1, i}$ at an angle strictly smaller than $\pi$ (see (124) above for a proper definition). We will then consider a Witten Laplacian with tangential Dirichlet boundary conditions on $\Gamma_{0} \cap \Gamma_{1, i}$ and normal Dirichlet boundary conditions on $\Gamma_{2, i}$. Moreover, in order not to introduce new generalized critical point on $\Gamma_{2, i}$, we would like to keep the property $\partial_{n} f>0$ on $\Gamma_{2, i}$ (where $n$ denotes the outward normal derivative to $\dot{\Omega}_{i}$ ). The aim of this section is indeed to define such a domain $\dot{\Omega}_{i}$.

A system of coordinates on a neighborhood of $\partial \Omega$.

Let us consider the function $f_{-}$defined on a neighborhood $V_{\partial \Omega}$ of $\partial \Omega$, as introduced in Proposition 70. Recall that $f_{-}(x)=0$ for $x \in \partial \Omega$ and that $V_{\partial \Omega}$ can be chosen such that $f_{-}>0$ on $V_{\partial \Omega} \backslash \partial \Omega$ and $\left|\nabla f_{-}\right| \neq 0$ on $V_{\partial \Omega}$. Let us now consider $\varepsilon>0$ such that

$$
V_{\varepsilon}=\left\{y \in \Omega, 0 \leq f_{-}(y) \leq \varepsilon\right\} \subset V_{\partial \Omega} .
$$

For any $x \in V_{\varepsilon}$, the dynamics

$$
\left\{\begin{array}{l}
\gamma_{x}^{\prime}(t)=-\frac{\nabla f_{-}}{\left|\nabla f_{-}\right|^{2}}\left(\gamma_{x}(t)\right) \\
\gamma_{x}(0)=x
\end{array}\right.
$$

is such that $\gamma_{x}\left(t_{x}\right) \in \partial \Omega$, where

$$
t_{x}=\inf \left\{t, \gamma_{x}(t) \notin \operatorname{int} V_{\varepsilon}\right\}
$$

This is indeed a consequence of the fact that $\frac{d}{d t} f_{-}\left(\gamma_{x}(t)\right)=-1<0$ on $\left[0, t_{x}\right)$. Notice that this also implies that $t_{x}$ describes $[0, \varepsilon]$ when $x$ describes $V_{\varepsilon}$.

The application

$$
\Gamma:\left\{\begin{aligned}
V_{\varepsilon} & \rightarrow \partial \Omega \times[-\varepsilon, 0] \\
x & \mapsto\left(\gamma_{x}\left(t_{x}\right),-t_{x}\right)
\end{aligned}\right.
$$

defines a $C^{\infty}$ diffeomorphism. The inverse application of $\Gamma$ is $\left(x^{\prime}, x_{d}\right) \in \partial \Omega \times[-\varepsilon, 0] \mapsto$ $\gamma_{x^{\prime}}\left(x_{d}\right)$. 
Definition 77. Let us assume that the hypothesis [H3] holds. Let us define the following system of coordinates for $x \in V_{\varepsilon}$ :

$$
\forall x \in V_{\varepsilon},\left(x^{\prime}(x), x_{d}(x)\right)=\left(\gamma_{x}\left(t_{x}\right),-t_{x}\right) \in \partial \Omega \times[-\varepsilon, 0] .
$$

Notice that, by construction ( since $\frac{d}{d t} f_{-}\left(\gamma_{x}(t)\right)=-1$ ),

$$
x_{d}(x)=-f_{-}(x) .
$$

Thus, in this system of coordinates, $\left\{x_{d}=0\right\}=\partial \Omega$ and $\left\{x_{d}<0\right\}=\Omega \cap V_{\varepsilon}$. We will sometimes need to use a local system of coordinates in $\partial \Omega$, that we will then denote by the same notation $x^{\prime}$. By using the same procedure as above, $\left(x^{\prime}, x_{d}\right)$ then defines a local system of coordinates. Let us make this precise. For $y \in \partial \Omega$, let us consider $x^{\prime}: V_{y} \rightarrow \mathbb{R}^{d-1}$ a smooth local system of coordinates in $\partial \Omega$, in a neighborhood $V_{y} \subset \partial \Omega$ of $y$. These coordinates are then extended in a neighborhood of $V_{y}$ in $\Omega$, as constant along the integral curves of $\gamma^{\prime}(t)=\frac{\nabla f_{-}}{\left|\nabla f_{-}\right|^{2}}(\gamma(t))$, for $t \in[0, \varepsilon]$. The function $x \mapsto\left(x^{\prime}, x_{d}\right)$ (where, we recall, $x_{d}(x)=-f_{-}(x)$ ) thus defines a smooth system of coordinates in a neighborhood $W_{y}$ of $y$ in $\bar{\Omega}$. In this system of coordinates, the metric tensor $G$ writes:

$$
G\left(x^{\prime}, x_{d}\right)=G_{d d}\left(x^{\prime}, x_{d}\right) d x_{d}^{2}+\sum_{i, j=1}^{d-1} G_{i j}\left(x^{\prime}, x_{d}\right) d x_{i} d x_{j},
$$

where $x^{\prime}=\left(x_{1}, \ldots, x_{d-1}\right)$. In particular if $\psi: V_{y} \rightarrow \mathbb{R}$ is a Lipschitz function which only depends on $x^{\prime}$, it holds a.e. on $V_{y}$ :

$$
\left|\nabla \psi\left(x^{\prime}, x_{d}\right)\right|=\left|\nabla\left(\left.\psi\right|_{\Sigma_{x_{d}}}\right)\left(x^{\prime}\right)\right|,
$$

where $\forall a>0, \Sigma_{a}:=\left\{x \in V_{\varepsilon}, x_{d}(x)=a\right\}$ is endowed with the Riemannian structure induced by the Riemannian structure in $\Omega$.

Definitions of the functions $\Psi_{i}, f_{+, i}$ and $f_{-, i}$.

Definition 78. Let us assume that the hypotheses [H1] and [H3] hold. Let us consider $z_{i}$ a local minimum of $\left.f\right|_{\partial \Omega}$ as introduced in hypothesis [H2]. Let us define on $\Omega$ the following Lipschitz functions

$$
\Psi_{i}(x):=d_{a}\left(x, z_{i}\right), \quad f_{+, i}:=\frac{\Psi_{i}+f-f\left(z_{i}\right)}{2} \text { and } f_{-, i}:=\frac{\Psi_{i}-\left(f-f\left(z_{i}\right)\right)}{2} .
$$

Owing to $\Psi_{i}(x)=d_{a}\left(x, z_{i}\right) \geq\left|f(x)-f\left(z_{i}\right)\right|$ for all $x \in \Omega$, the functions $f_{ \pm, i}$ are non negative and

$$
f=f\left(z_{i}\right)+f_{+, i}-f_{-, i} \text { and } \Psi_{i}=f_{+, i}+f_{-, i} \text { on } \Omega .
$$

Let $\Gamma_{1, i} \subset B_{z_{i}}$ be an open smooth $d-1$ dimensional manifold with boundary such that $z_{i} \in \Gamma_{1, i}$ and $\overline{\Gamma_{1, i}} \subset B_{z_{i}}$. From Proposition 71 , there exists a neighborhood of $\overline{\Gamma_{1, i}}$ in $\bar{\Omega}$, denoted $V_{\Gamma_{1, i}}$, such that $\overline{\partial V_{\Gamma_{1, i}} \cap \partial \Omega} \subset B_{z_{i}}$ and for all $x \in V_{\Gamma_{1, i}}$,

$$
\Psi_{i}(x)=\Phi(x)-f\left(z_{i}\right)
$$

where $\Phi$ is the solution to the eikonal equation in a neighborhood of the boundary (see Proposition 67. Notice that it implies that on $V_{\Gamma_{1, i}}$ the function $f_{-, i}$ coincides with 
the function $f_{-}$defined in Proposition 70 on $V_{\partial \Omega} \cap V_{\Gamma_{1, i}}$. Moreover, it implies that the functions $f_{ \pm, i}$ are $C^{\infty}$ on $V_{\Gamma_{1, i}}$ and one has:

$$
\text { on } V_{\Gamma_{1, i}} \cap \partial \Omega, \quad f_{+, i}=f-f\left(z_{i}\right), \quad f_{-, i}=0, \quad \partial_{n} f_{+, i}=0, \text { and } \partial_{n} f_{-, i}=-\partial_{n} f,
$$

where $n$ is the unit outward normal to $\Omega$. Therefore, as in Proposition 70, up to choosing a smaller neighborhood $V_{\Gamma_{1, i}}$ of $\overline{\Gamma_{1, i}}$ in $\bar{\Omega}$, the function $f_{-, i}$ is positive on $V_{\Gamma_{1, i}} \backslash \partial \Omega$ and such that

$$
\left|\nabla f_{-, i}\right| \neq 0 \text { in } V_{\Gamma_{1, i}} .
$$

Besides, since $\left|\nabla \Psi_{i}\right|=|\nabla f|$ in $V_{\Gamma_{1, i}}$, one has

$$
\nabla f_{+, i} \cdot \nabla f_{-, i}=0 \text { in } V_{\Gamma_{1, i}}
$$

and thus

$$
\left|\nabla \Psi_{i}\right|^{2}=|\nabla f|^{2}=\left|\nabla f_{+, i}\right|^{2}+\left|\nabla f_{-, i}\right|^{2} \text { in } V_{\Gamma_{1, i}} .
$$

In the following, we will assume in addition that $V_{\Gamma_{1, i}}$ is sufficiently small so that the system of coordinates $\left(x^{\prime}, x_{d}\right)$ introduced in Definition 77 is well defined on $V_{\Gamma_{1, i}}$. A consequence of (139) is that $\frac{d}{d t} f_{+, i}\left(\gamma_{x}(t)\right)=0$, where $\gamma_{x}$ satisfies (135). Thus, in the system of coordinates $\left(x^{\prime}, x_{d}\right)$, the functions $f_{+, i}, \Psi_{i}$ and $f$ write:

$$
\begin{aligned}
& f_{+, i}\left(x^{\prime}, x_{d}\right)=f_{+, i}\left(x^{\prime}, 0\right), \Psi_{i}\left(x^{\prime}, x_{d}\right)=f_{+, i}\left(x^{\prime}, 0\right)-x_{d} \\
& \text { and } f\left(x^{\prime}, x_{d}\right)=f\left(z_{i}\right)+f_{+, i}\left(x^{\prime}, 0\right)+x_{d} .
\end{aligned}
$$

Notice that by construction

$$
\forall x \in V_{\Gamma_{1, i}},\left|\nabla f_{+, i}\right|(x)=0 \Longleftrightarrow x^{\prime}(x)=x^{\prime}\left(z_{i}\right) .
$$

Indeed, $f_{+, i}\left(x^{\prime}, x_{d}\right)=f_{+, i}\left(x^{\prime}, 0\right)$ and $x^{\prime} \mapsto f_{+, i}\left(x^{\prime}, 0\right)=f\left(x^{\prime}, 0\right)-f\left(z_{i}\right)$ has a single critical point at $x^{\prime}\left(z_{i}\right)$.

Strongly stable domain in $B_{z_{i}}$.

In order to build an appropriate domain $\dot{\Omega}_{i}$, we will need to define $\Gamma_{1, i} \subset B_{z_{i}}$ as a strongly stable domain, as defined now.

Definition 79. A smooth open set $A \subset \partial \Omega$ is called strongly stable if

$$
\forall \sigma \in \partial A, \quad\left\langle\nabla\left(\left.f\right|_{\partial \Omega}\right)(\sigma), n_{\sigma}(A)\right\rangle_{T_{\sigma} \partial \Omega}>0,
$$

where $n_{\sigma}(A) \in T_{\sigma} \partial \Omega$ denotes the outward normal to $A$ at $\sigma \in \partial A$.

Notice that $\nabla\left(\left.f\right|_{\partial \Omega}\right)=\nabla_{T} f=\nabla f_{+, i}$ (this is due to the fact that on $B_{z_{i}}$, one has $f-f\left(z_{i}\right)=\Psi_{i}$ and thus $\left.\nabla_{T} f=\nabla_{T} \Psi_{i}\right)$. Thus, the strong stability condition appearing in Definition 79 is equivalent to

$$
\forall \sigma \in \partial A, \quad \partial_{n_{\sigma}(A)} f_{+, i}(\sigma)>0 .
$$

The name "stable" is justified by the following: if $A \subset \partial \Omega$ is strongly stable, then for any curve satisfying for all $t>0, \gamma^{\prime}(t)=-\nabla\left(\left.f\right|_{\partial \Omega}\right)(\gamma(t))$ with $\gamma(0) \in \bar{A}$, one has for all $t \geq 0, \gamma(t) \in \bar{A}$.

The following proposition will be needed to get the existence of an arbitrary large and strongly stable domain in $B_{z_{i}}$. 
Proposition 80. Let us assume that the hypotheses [H1] and [H2] hold. For all compact sets $K \subset B_{z_{i}}$ there exists a $C^{\infty}$ open domain $A$ which is strongly stable in the sense of Definition 79, simply connected and such that $K \subset A$ and $\bar{A} \subset B_{z_{i}}$.

Proof. For the ease of notation, we drop the subscript $i$ in the proof. One will first construct the set $A$. Then it will be proven that $A$ has the stated properties. For $a>0$, let us define

$$
L_{a}:=\left.f\right|_{\partial \Omega} ^{-1}([f(z), f(z)+a)) \cap B_{z} .
$$

For a fixed $a>0$ small enough $L_{a}$ is a $C^{\infty}$ simply connected open set (which contains $z$ ) with boundary the level set $\left.f\right|_{\partial \Omega} ^{-1}(\{f(z)+a\})$. The domain $L_{a}$ is $C^{\infty}$ since $f$ is $C^{\infty}$.

Let us define for $x \in B_{z}$ the curves $\gamma_{x}$ by

$$
\gamma_{x}^{\prime}(t)=\left.\nabla f\right|_{\partial \Omega}\left(\gamma_{x}(t)\right), \quad \gamma_{x}(0)=x .
$$

For any $x \in \partial L_{a}$, for all $t>0, \gamma_{x}(-t) \in L_{a}$ since $t \geq\left. 0 \mapsto f\right|_{\partial \Omega}\left(\gamma_{x}(-t)\right)$ is decreasing $\left(\left.\frac{d}{d t} f\right|_{\partial \Omega}\left(\gamma_{x}(-t)\right)=-\left.|\nabla f|_{\partial \Omega}\left(\gamma_{x}(-t)\right)\right|^{2}\right.$ and $\left.\left.f\right|_{\partial \Omega}\left(\gamma_{x}(0)\right)=a\right)$. Let us now define for $T>0$

$$
A_{T}:=\left\{\gamma_{x}(t), x \in \partial L_{a}, t \in[0, T)\right\} \cup L_{a} \subset B_{z} .
$$

One clearly has $A_{T} \subset A_{T^{\prime}}$ if $T<T^{\prime}$. One claims that $A_{T}$ is a $C^{\infty}$ simply connected open set which satisfies

$$
\forall \sigma \in \partial A_{T},\left.\quad \partial_{n_{\sigma}\left(A_{T}\right)} f\right|_{\partial \Omega}(\sigma)>0 .
$$

Let us first prove that $A_{T}$ is $C^{\infty}$. One has $\partial A_{T}=\left\{\gamma_{x}(T), x \in \partial L_{a}\right\}$. The boundary of $A_{T}$ is thus a $C^{\infty}$ homotopy of $\partial L_{a}$ where the homotopy function is

$$
H(t, x)=\gamma_{x}(t)
$$

Additionally since this homotopy is with values in $B_{z}$ and since $L_{a}$ is simply connected (because $L_{a}$ can be asymptotically retracted on $z$ in the sense that for all $x \in L_{a}$, $\left.\lim _{t \rightarrow-\infty} H(t, x)=z\right), A_{T}$ is simply connected. Let us prove that $A_{T}$ is open. Let us denote by $d_{\partial \Omega}$ the geodesic distance in $\partial \Omega$. Let $x_{0} \in A_{T} \backslash \bar{L}_{a}$. There exists a time $t_{0} \in(0, T)$ such that $\gamma_{x_{0}}\left(-t_{0}\right) \in L_{a}$. Let us define $\varepsilon_{0}=d_{\partial \Omega}\left(\gamma_{x_{0}}\left(-t_{0}\right), \partial L_{a}\right) / 2>0$. Since the mapping $y \mapsto \gamma_{y}\left(-t_{0}\right)$ is $C^{\infty}$, there exists $\varepsilon_{1}>0$ such that if $d_{\partial \Omega}(x, y) \leq \varepsilon_{1}$ then $d_{\partial \Omega}\left(\gamma_{y}\left(-t_{0}\right), \gamma_{x_{0}}\left(-t_{0}\right)\right) \leq \varepsilon_{0} / 2$ and thus $\gamma_{y}\left(-t_{0}\right) \in L_{a}$. Moreover, since $B_{z} \backslash \bar{L}_{a}$ is open, it can be assumed, taking maybe $\varepsilon_{1}>0$ smaller, that $B_{\partial \Omega}\left(x_{0}, \varepsilon_{1}\right) \subset B_{z} \backslash \bar{L}_{a}$. Then, by continuity, for all $y \in B_{\partial \Omega}\left(x_{0}, \varepsilon_{1}\right)$, there exists $t_{0}(y) \in\left(0, t_{0}\right) \subset(0, T)$ such that $\gamma_{y}\left(-t_{0}(y)\right) \in \partial L_{a}$, which implies that $y \in A_{T} \backslash \bar{L}_{a}$. Thus $A_{T} \backslash \bar{L}_{a}$ is open. In addition, since $L_{a}$ is open and since $\bar{L}_{a} \subset A_{T}$, one has that $\operatorname{int}\left(A_{T}\right)=\operatorname{int}\left(A_{T} \backslash \bar{L}_{a}\right) \cup \bar{L}_{a}=$ $\left(A_{T} \backslash \bar{L}_{a}\right) \cup \bar{L}_{a}=A_{T}$. Therefore the set $A_{T}$ is open.

Let us now prove that $A_{T}$ is strongly stable (see Definition 79. By construction, $A_{T}$ is stable for the dynamics $\gamma^{\prime}=-\left.\nabla f\right|_{\partial \Omega}(\gamma)$ and thus one has

$$
\forall \sigma \in \partial A_{T},\left.\quad \partial_{n_{\sigma}\left(A_{T}\right)} f\right|_{\partial \Omega}(\sigma) \geq 0 .
$$

Let us defined now the function

$$
\Upsilon: x \in B_{z} \backslash \bar{L}_{a} \mapsto\left(x^{\prime}, t\right) \in \partial L_{a} \times \mathbb{R}_{+} \text {s.t } \gamma_{x^{\prime}}(t)=x .
$$


Notice that $\Upsilon$ is a $C^{\infty}$ diffeomorphism from $B_{z}$ onto its range, and let us denote $F:=$ $\Upsilon^{-1}$ its inverse function $\left(F\left(x^{\prime}, t\right)=\gamma_{x^{\prime}}(t)\right)$. Assume that there exists $x \in A_{T}$ such that $\left.\partial_{n_{x}\left(A_{T}\right)} f\right|_{\partial \Omega}(x)=0$ and let $\left(x^{\prime}, T\right)=\Upsilon(x)$. This implies that $\left.\nabla f\right|_{\partial \Omega}(x) \in T_{x} \partial A_{T}$ and thus $\partial_{t} F\left(x^{\prime}, T\right) \in T_{x} \partial A_{T}$. Furthermore $\operatorname{Ran}\left(d_{x^{\prime}} F(., T)\right)=T_{x} \partial A_{T}$ and thus $d_{\left(x^{\prime}, T\right)} F$ is not invertible which contradicts the fact that $F$ is a diffeomorphism.

It remains to prove that for any compact set $K \subset B_{z}$, there exists $T>0$ such that $K \subset A_{T}$. One has

$$
B_{z}=\bigcup_{T>0} A_{T}
$$

Indeed, if $x \in \bar{L}_{a}, x \in A_{T}$ for all $T>0$ and if $x \in B_{z} \backslash \bar{L}_{a}, \lim _{t \rightarrow \infty} \gamma_{x}(-t)=z$ and thus there exists $s>0$ such that $\gamma_{x}(-s) \in \partial L_{a}$ which implies that $x \in A_{s}$. Let $K \subset B_{z}$ be a compact set. Then $K \subset \bigcup_{T>0} A_{T}$ and thus by compactness there exists a sequence $\left(T_{j}\right)_{j=1, \ldots, N} \subset \mathbb{R}^{N}$, with $0<T_{1}<\ldots<T_{m}$ such that $K \subset \bigcup_{j=1}^{m} A_{T_{j}}=A_{T_{m}}$. This concludes the proof.

Construction of the domain $\dot{\Omega}_{i}$.

In this section, we introduce the domain $\dot{\Omega}_{i}$ (associated with $z_{i}$ ) on which the auxiliary Witten Laplacian with mixed tangential-normal Dirichlet boundary conditions is constructed.

Proposition 81. Let us assume that the hypotheses [H1], [H2] and [H3] hold. Let us fix a neighborhood $\Omega_{0}$ of $x_{0}$ (the global minimum of $f$ in $\Omega$ ) such that

$$
\partial_{n} f<0 \text { on } \Gamma_{0}:=\partial \Omega_{0}
$$

where $n$ denotes the outward normal to $\Omega \backslash \Omega_{0}$ on $\Gamma_{0}$. Let us consider a critical point $z_{i}$ of $\left.f\right|_{\partial \Omega}$. Then there exists a smooth open subset $\Gamma_{1, i}$ of $B_{z_{i}}$ containing $z_{i}$ and arbitrarily large in $B_{z_{i}}$, a neighborhood $V_{\Gamma_{1, i}}$ of $\overline{\Gamma_{1, i}}$ in $\bar{\Omega}$ such that $\overline{V_{\Gamma_{1, i}} \cap \partial \Omega} \subset B_{z_{i}}$ and a Lipschitz subset $\dot{\Omega}_{i}$ of $\Omega \backslash \Omega_{0}$ which are such that the following properties are satisfied:

\section{Following Proposition 71 .}

$$
\forall x \in V_{\Gamma_{1, i}}, d_{a}\left(x, z_{i}\right)=\Phi(x)-f\left(z_{i}\right)
$$

where $\Phi$ is the solution to the eikonal equation (107);

2. The system of coordinates $\left(x^{\prime}, x_{d}\right)$ is defined on $V_{\Gamma_{1, i}}$, see Definition 77 ;

3. $\partial \dot{\Omega}_{i}$ is composed of two connected components: $\Gamma_{0}$ and $\overline{\Gamma_{1, i}} \cup \overline{\Gamma_{2, i}}$, where $\Gamma_{2, i}$ is an open subset of $\partial \dot{\Omega}_{i}, \Gamma_{1, i} \cap \Gamma_{2, i}=\emptyset$, and $\Gamma_{2, i}$ is such that there exist two disjoint $C^{\infty}$ open subsets $\Gamma_{2, i}^{1}$ and $\Gamma_{2, i}^{2}$ of $\partial \dot{\Omega}_{i}$ such that

$$
\overline{\Gamma_{2, i}}=\overline{\Gamma_{2, i}^{1}} \cup \overline{\Gamma_{2, i}^{2}} ;
$$

4. $\Gamma_{1, i}$ and $\Gamma_{2, i}$ meet at an angle smaller than $\pi$, see (124) for a precise definition;

5. It holds,

$$
\forall x \in \partial \dot{\Omega}_{i} \backslash\left(\left(\overline{\Gamma_{2, i}^{1}} \cap \overline{\Gamma_{2, i}^{2}}\right) \cup\left(\overline{\Gamma_{1, i}} \cap \overline{\Gamma_{2, i}}\right) \cup \Gamma_{0}\right), \partial_{n} f(x)>0
$$

where $n$ is the outward normal to $\dot{\Omega}_{i}$; 
6. It holds,

$$
\forall x \in \Gamma_{2, i} \cap V_{\Gamma_{1, i}}, \partial_{n} f_{+, i}(x)>0 ;
$$

7. Moreover, for all $\delta>0, \dot{\Omega}_{i}$ (and $\Omega_{0}$ ) can be chosen such that

$$
\sup \left\{d_{e}(x, y), x \in \Gamma_{2, i}, y \in B_{z_{i}}^{c}\right\} \leq \delta
$$

and

$$
\sup \left\{d_{e}\left(x_{0}, x\right), x \in \Gamma_{0}\right\} \leq \delta
$$

where, we recall, $d_{e}$ denotes the geodesic distance for the Euclidean metric on $\bar{\Omega}$.

We refer to Figure 10 for a schematic representation of $\dot{\Omega}_{i}$.

Proof. The domain $\dot{\Omega}_{i} \subset \Omega$ is built as follows. First, let us fix a neighborhood $\Omega_{0}$ of $x_{0}$ such that 145 is satisfied and

$$
\partial_{n} f<0 \text { on } \Gamma_{0}=\partial \Omega_{0}
$$

where $n$ denotes the outward normal to $\Omega \backslash \Omega_{0}$ on $\Gamma_{0}$ (this can be done for example by considering $\Omega_{0}=\left\{x, f(x)<f\left(x_{0}\right)+\eta\right\}$ for some positive $\left.\eta\right)$. Second, let us consider a smooth subset $\Gamma_{1, i}$ of $B_{z_{i}}$ which can be as large as needed in $B_{z_{i}}$, and which is strongly stable (see Proposition 80 for the existence of such a set):

$$
\left\langle\left.\nabla f\right|_{\partial \Omega}, n\left(\Gamma_{1, i}\right)\right\rangle_{T \partial \Omega}>0 \text { on } \partial \Gamma_{1, i} \text { and thus } \partial_{n\left(\Gamma_{1, i}\right)} f_{+, i}>0 \text { on } \partial \Gamma_{1, i},
$$

where $n\left(\Gamma_{1, i}\right)$ denotes the outward normal derivative of $\Gamma_{1, i}$ (see Definition 79 and (141)).

Once $\Gamma_{1, i}$ is fixed, the existence of a neighborhood $V_{\Gamma_{1, i}}$ of $\overline{\Gamma_{1, i}}$ in $\bar{\Omega}$ such that $\overline{V_{\Gamma_{1, i}} \cap \partial \Omega} \subset B_{z_{i}}$ and such that items 1 and 2 are fulfilled is a direct consequence of Proposition 71 .

Let us now consider the system of coordinates $\left(x^{\prime}, x_{d}\right)$ introduced in Definition 77 . Let $V_{\partial \Gamma_{1, i}} \subset \partial \Omega$ denotes a neighborhood of $\partial \Gamma_{1, i}$ in $\partial \Omega$ and

$$
V_{\partial \Gamma_{1, i}}^{+}=\overline{V_{\partial \Gamma_{1, i}} \cap \Gamma_{1, i}^{c}}
$$

The domain $\dot{\Omega}_{i}$ is then defined as follows:

$$
\dot{\Omega}_{i}=\Omega \backslash\left(\overline{\Omega_{0}} \cup\left\{x=\left(x^{\prime}, x_{d}\right), x^{\prime} \in V_{\partial \Gamma_{1, i}}^{+} \text {such that } x_{d}(x) \in\left[-\varphi\left(x^{\prime}\right), 0\right]\right\}\right)
$$

where $\varphi: V_{\partial \Gamma_{1, i}}^{+} \rightarrow \mathbb{R}_{+}$is a smooth function such that

$$
\exists \varepsilon>0, \forall x^{\prime} \in \partial \Gamma_{1, i}, \varphi\left(x^{\prime}\right) \geq \varepsilon,
$$

see Figure 11 for a schematic representation. Notice that by construction, $\dot{\Omega}_{i}$ is a connected Lipschitz subset of $\Omega$ and, denoting by $\Gamma_{2, i}=\partial \dot{\Omega}_{i} \backslash\left(\overline{\Gamma_{1, i}} \cup \Gamma_{0}\right)$, item 3 is satified. For each point $z \in \partial \Gamma_{1, i}$, there is a small neighborhood $\mathcal{V}$ of $z$ such that

$$
\mathcal{V} \cap \Gamma_{2, i} \subset\left\{x=\left(x^{\prime}, x_{d}\right), x^{\prime} \in \partial \Gamma_{1, i} \text { and } x_{d}(x) \in(-\eta, 0]\right\},
$$

for some $\eta \in(0, \varepsilon)$. By choosing $\Gamma_{1, i}$ sufficiently large in $B_{z_{i}}$, and $\varphi$ such that $\max \varphi$ is sufficiently small, (144) is satisfied. This concludes the verification of item 7 .

For each point $y \in \partial \Gamma_{1, i}$, it is possible to construct locally a normal system of coodinate $x^{\prime}=x_{T}=\left(x_{T, 1}, x_{T, 2}, \ldots, x_{T, d-1}\right)$ in a neighborhood $V_{y}$ of $y$ in $\partial \Omega$, such 
that $\Gamma_{1, i} \cap V_{y}=\left\{x \in V_{y}, x_{T, 1}(x) \leq 0\right\}, V_{\partial \Gamma_{1, i}}^{+} \cap V_{y}=\left\{x \in V_{y}, x_{T, 1}(x) \geq 0\right\}$ and $\partial \Gamma_{1, i} \cap V_{y}=\left\{x \in V_{y}, x_{T, 1}(x)=0\right\}$. As explained after Definition 77 , by extending this system of coordinate inside $\Omega$ as constant along the curve associated with the vector field $\frac{\nabla f_{-, i}}{\left|\nabla f_{-, i}\right|^{2}}, x \mapsto\left(x^{\prime}(x), x_{d}(x)\right)$ then defines a local system of coordinates in a neighborhood $W_{y}$ of $y$ in $\bar{\Omega}$. For all $x \in \partial \Gamma_{1, i}$, the vector $n_{z}\left(\Gamma_{1}\right)=\frac{\nabla x_{T, 1}(x)}{\left|\nabla x_{T, 1}(x)\right|}$ is the outward normal vector to $\Gamma_{1, i}$ on $\partial \Gamma_{1, i}$. By a compactness argument, one gets that $\partial \Gamma_{1, i} \subset \cup_{k=1}^{K} \mathcal{V}_{y_{k}}$ for a finite number of points $y_{k} \in \partial \Gamma_{1, i}$. See Figure 12 for a schematic representation of the function $\varphi$ in this system of coordinates.

Let us now look at the boundary of $\dot{\Omega}_{i}$ in a neighborhood of $\partial \Gamma_{1, i}$ (see Figure 11 ). For $\sigma \in \partial \dot{\Omega}_{i}$, let us denote by $n_{\sigma}\left(\dot{\Omega}_{i}\right)$ the unit outward normal to $\dot{\Omega}_{i}$. Let us show that for all $z \in \partial \Gamma_{1, i}$

$$
\lim _{\sigma \rightarrow z} n_{\sigma}\left(\dot{\Omega}_{i}\right)=n_{z}\left(\Gamma_{1, i}\right)
$$

where the limit is taken for $\sigma \in \Gamma_{2, i}$. Let us prove (148). For any point $z \in \partial \Gamma_{1, i}$, there is a small neighborhood $\mathcal{V}$ of $z$ in $\bar{\Omega}$ such that the system of coordinates $\left(x_{T}, x_{d}\right)$ introduced above is well defined. In this system of coordinates,

$$
\partial \dot{\Omega}_{i} \cap \mathcal{V} \cap \Gamma_{2, i} \subset\left\{x \in \mathcal{V}, x_{T, 1}(x)=0 \text { and } x_{d}(x) \in\left[-\varphi\left(x^{\prime}\left(x_{T}(x)\right)\right), 0\right]\right\} .
$$

Moreover, the outward normal to $\dot{\Omega}_{i}$ on this subset is $n\left(\dot{\Omega}_{i}\right)=\frac{\nabla x_{T, 1}}{\left|\nabla x_{T, 1}\right|}$ and thus, by construction, for all $z \in \partial \Gamma_{1, i}, 148$ holds.

As a consequence of 148, the two submanifolds $\Gamma_{1, i}$ and $\Gamma_{2, i}$ meet at an angle smaller than $\pi$ (see (124) and Figure 13). This shows that item 4 is satisfied. Moreover, using (146), one has: for all $z \in \partial \Gamma_{1, i}$,

$$
\lim _{\sigma \rightarrow z} \nabla f_{+, i}(\sigma) \cdot n_{\sigma}\left(\dot{\Omega}_{i}\right)=\nabla f_{+, i}(z) \cdot n_{z}\left(\Gamma_{1, i}\right)>0,
$$

and

$$
\lim _{\sigma \rightarrow z} \nabla f(\sigma) \cdot n_{\sigma}\left(\dot{\Omega}_{i}\right)=\nabla f(z) \cdot n_{z}\left(\Gamma_{1, i}\right)>0,
$$

where the limits are taken for $\sigma \in \Gamma_{2, i}$. Thus, up to choosing $\varphi$ with $\max \varphi$ sufficiently small, it is possible to build $\dot{\Omega}_{i}$ such that (see Figure 11)

$$
\forall x \in \Gamma_{2, i} \text { such that } x^{\prime}(x) \in \partial \Gamma_{1, i}, \partial_{n} f_{+, i}(x)>0 \text { and } \partial_{n} f(x)>0,
$$

where $n$ here denotes the outward normal to $\dot{\Omega}_{i}$. Finally, by using $(149)$ and since $\partial_{n} f>0$ on $\partial \Omega$, up to choosing $\varphi$ with $\max \varphi$ sufficiently small, it is possible to build $\dot{\Omega}_{i}$ such that (see Figure 11 )

$$
\forall x \in \partial \dot{\Omega}_{i} \backslash\left(\left(\overline{\Gamma_{2, i}^{1}} \cap \overline{\Gamma_{2, i}^{2}}\right) \cup\left(\overline{\Gamma_{1, i}} \cap \overline{\Gamma_{2, i}}\right) \cup \Gamma_{0}\right), \partial_{n} f(x)>0
$$

where $n$ again denotes the outward normal to $\dot{\Omega}_{i}$. This is item 5 , and this concludes the proof of Proposition 81 .

Definition 82. Let us assume that the hypotheses [H1], [H2] and [H3] hold. Let us consider a critical point $z_{i}$ of $\left.f\right|_{\partial \Omega}$. In the following, we denote by

$$
\mathcal{S}_{M, i}:=\left\{\dot{\Omega}_{i}, \Gamma_{0}, \Gamma_{1, i}, \Gamma_{2, i}, V_{\Gamma_{1, i}}\right\}
$$

an ensemble of sets satisfying the requirements of Proposition 81 . 


$$
\Gamma_{1, i} \text { in } \partial \Omega
$$
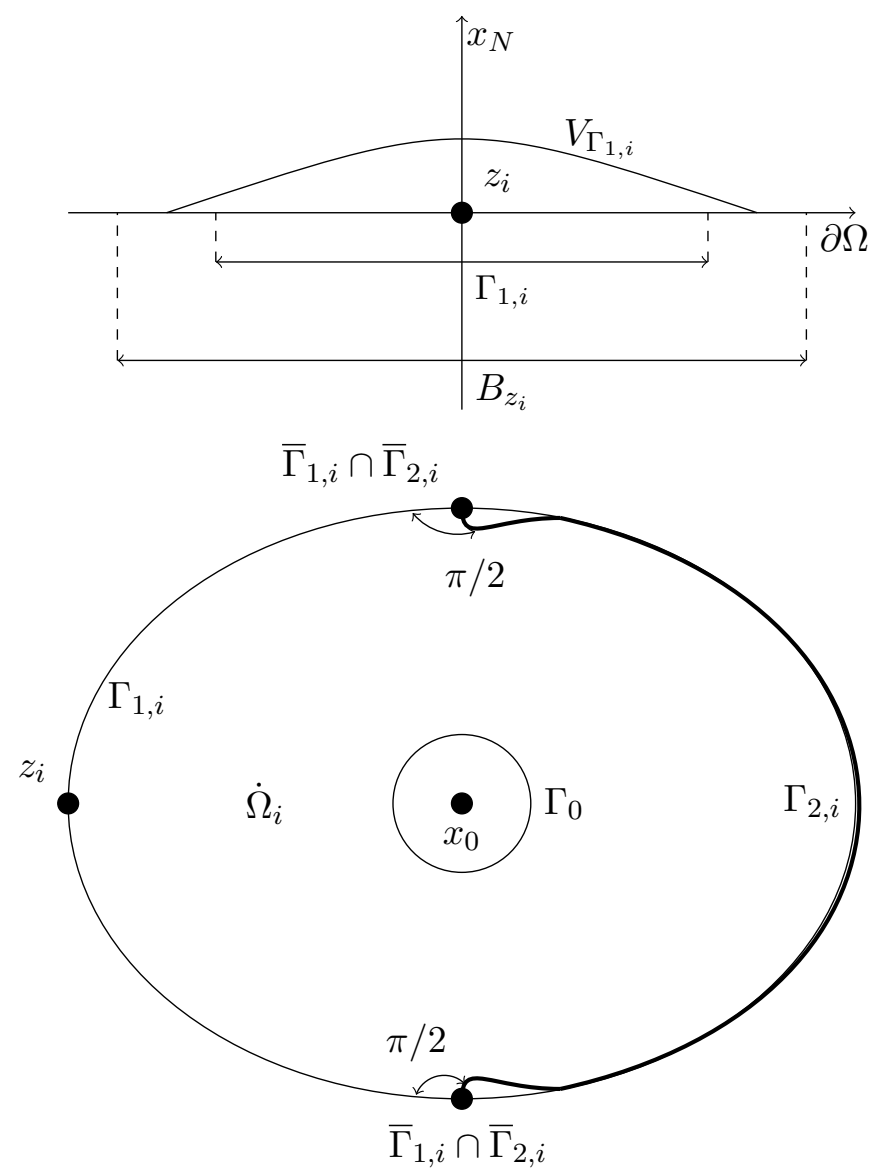

Figure 10: The ensemble of sets $\mathcal{S}_{M, i}=\left\{\dot{\Omega}_{i}, \Gamma_{0}, \Gamma_{1, i}, \Gamma_{2, i}, V_{\Gamma_{1, i}}\right\}$ associated with a critical point $z_{i}$ of $\left.f\right|_{\partial \Omega}$.

In the following, in order to reduce the amount of notation, the index $i$ will sometimes be omitted. Thus, we will denote

$$
z=z_{i}, \Gamma_{1}=\Gamma_{1, i}, \Gamma_{2}=\Gamma_{2, i}, \dot{\Omega}=\dot{\Omega}_{i}, V_{\Gamma_{1}}=V_{\Gamma_{1, i}}, \Psi=\Psi_{i}, f_{+}=f_{+, i} \text { and } f_{-}=f_{-, i}
$$

We shall warn the reader whenever the index $i$ is dropped.

\subsubsection{On the spectrum of the Witten Laplacian $\Delta_{f, h}^{M}\left(\dot{\Omega}_{i}\right)$}

Troughout this section, one assumes [H1], [H2] and [H3]. In this section, we introduce a Witten Laplacian with mixed tangential and normal Dirichlet boundary conditions, associated with the critical point $z_{i}$. Let $\mathcal{S}_{M, i}:=\left\{\dot{\Omega}_{i}, \Gamma_{0}, \Gamma_{1, i}, \Gamma_{2, i}, V_{\Gamma_{1, i}}\right\}$ be an ensemble of sets associated with $z_{i}$, see Definition 82

Let us now consider the Witten Laplacian $\Delta_{f, h}^{M}$ on $\dot{\Omega}_{i}$ with homogeneous Dirichlet tangential boundary conditions on

$$
\Gamma_{T}=\Gamma_{0} \cup \Gamma_{1, i}
$$




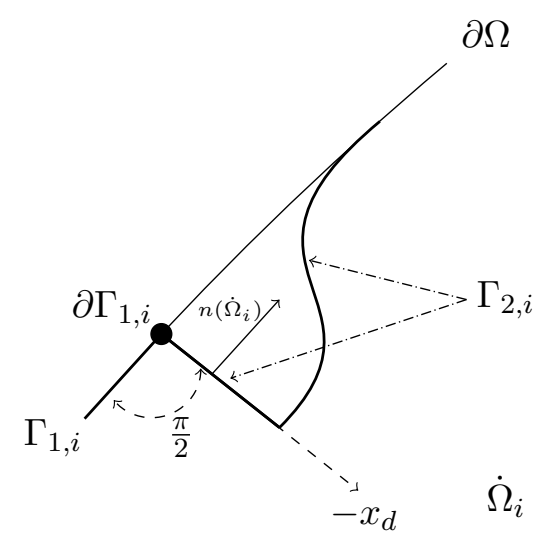

Figure 11: $\dot{\Omega}_{i}$ near $\partial \Gamma_{1, i}$.

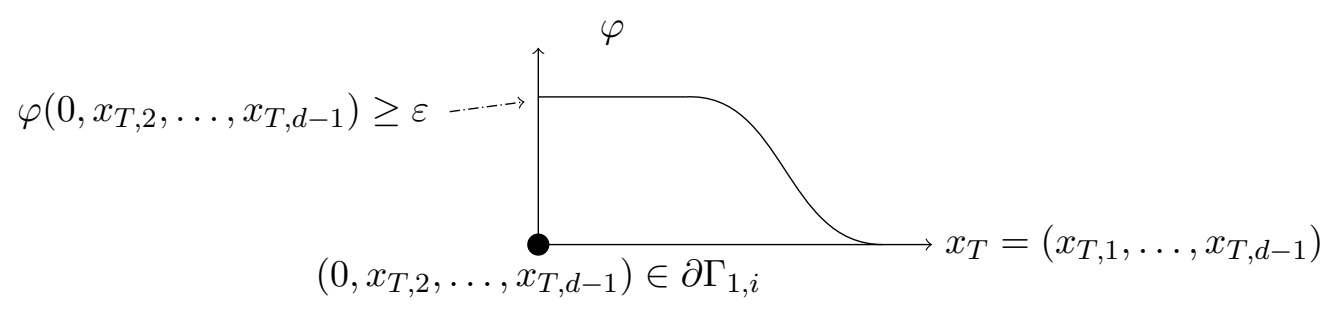

Figure 12: The function $x_{T} \in \mathbb{R}^{d-1} \mapsto \varphi\left(x_{T}\right)$.

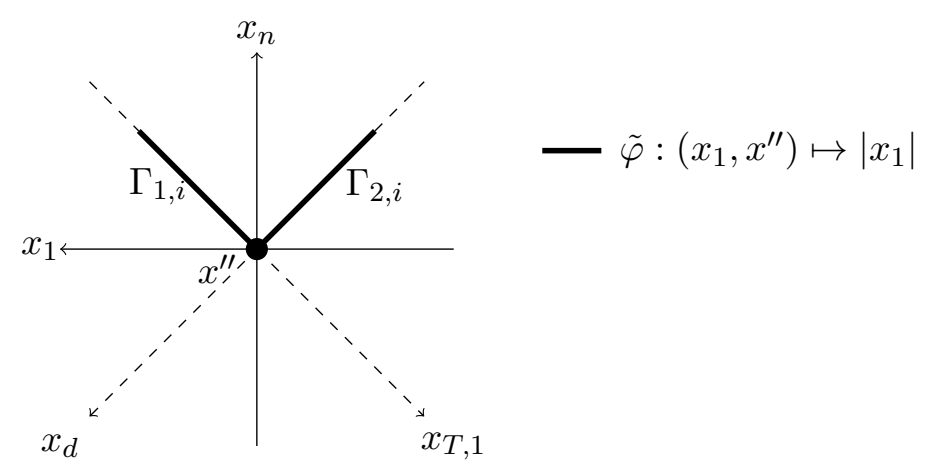

Figure 13: The sets $\Gamma_{1, i}$ and $\Gamma_{2, i}$ meet at an angle smaller than $\pi$, according to 124): take $x_{1}=\frac{x_{d}-x_{T, 1}}{2}, x_{n}=-\frac{x_{d}+x_{T, 1}}{2}, x^{\prime \prime}=\left(x_{T, 2}, \ldots, x_{T, d-1}\right)$ and $\tilde{\varphi}\left(x_{1}, x^{\prime \prime}\right)=\left|x_{1}\right|$.

and homogeneous Dirichlet normal boundary conditions on

$$
\Gamma_{N}=\Gamma_{2, i}
$$

as defined at the end of Section 4.1.1 (see 127)-129). The main result of this section concerns the spectrum of the operator $\Delta_{f, h}^{M}\left(\dot{\Omega}_{i}\right)$.

Proposition 83. Let us assume that the hypotheses [H1], [H2] and [H3] hold. Let $\Delta_{f, h}^{M,(p)}\left(\dot{\Omega}_{i}\right)$ be the unbounded nonnegative selfadjoint operator on $L^{2}\left(\dot{\Omega}_{i}\right)$ defined by (127) and with domain (129) with $\Gamma_{T}=\Gamma_{1, i} \cup \Gamma_{0}$ and $\Gamma_{N}=\Gamma_{2, i}$. One has:

(i) The operator $\Delta_{f, h}^{M,(p)}\left(\dot{\Omega}_{i}\right)$ has compact resolvent. 
(ii) For any eigenvalue $\lambda_{p}$ of $\Delta_{f, h}^{M,(p)}\left(\dot{\Omega}_{i}\right)$ and associated eigenform $u_{p} \in D\left(\Delta_{f, h}^{M,(p)}\left(\dot{\Omega}_{i}\right)\right)$, one has $d_{f, h} u_{p} \in D\left(\Delta_{f, h}^{M,(p+1)}\right)$ and $d_{f, h}^{*} u_{p} \in D\left(\Delta_{f, h}^{M,(p-1)}\right)$, with

$$
d_{f, h} \Delta_{f, h}^{M,(p)} u_{p}=\Delta_{f, h}^{M,(p+1)} d_{f, h} u_{p}=\lambda_{p} d_{f, h} u_{p}
$$

and

$$
d_{f, h}^{*} \Delta_{f, h}^{M,(p)}\left(\dot{\Omega}_{i}\right) u_{p}=\Delta_{f, h}^{M,(p-1)}\left(\dot{\Omega}_{i}\right) d_{f, h}^{*} u_{p}=\lambda_{p} d_{f, h}^{*} u_{p} .
$$

If in addition $\lambda_{p} \neq 0$, either $d_{f, h} u_{p}$ or $d_{f, h}^{*} u_{p}$ is nonzero.

(iii) There exist $c>0$ and $h_{0}>0$ such that for any $p \in\{0, \ldots, n\}$ and $h \in\left(0, h_{0}\right)$,

$$
\operatorname{dim} \operatorname{Ran} \pi_{\left[0, c h^{\frac{3}{2}}\right)}\left(\Delta_{f, h}^{M,(p)}\left(\dot{\Omega}_{i}\right)\right)=\delta_{1, p} \quad \text { and } \quad \operatorname{Sp}\left(\Delta_{f, h}^{M,(1)}\left(\dot{\Omega}_{i}\right)\right) \cap\left[0, c h^{\frac{3}{2}}\right)=\{0\},
$$

where $\delta$ is the Kronecker delta: $\delta_{1, p}=1$ iff $p=1$.

Proof. Since the criticial point $z_{i}$ is fixed, for the ease of notation, we drop the subscript $i$ in the proof.

The point $(i)$ follows from the compactness of the embedding $H^{\frac{1}{2}}(\dot{\Omega}) \hookrightarrow L^{2}(\dot{\Omega})$ (since additionally $D\left(\mathcal{Q}_{f, h}^{M}(\dot{\Omega})\right) \hookrightarrow H^{\frac{1}{2}}(\dot{\Omega})$ is continuous according to Proposition 75 . The point $(i i)$ is then a straightforward consequence of the characterization of the domain of $\Delta_{f, h}^{M,(p)}(\dot{\Omega})$ together with 128 . The statement in the case $\lambda_{p} \neq 0$ follows from

$0 \neq \lambda_{p}\left\langle u_{p}, u_{p}\right\rangle_{L^{2}(\dot{\Omega})}=\left\langle\Delta_{f, h}^{M,(p)}(\dot{\Omega}) u_{p}, u_{p}\right\rangle_{L^{2}(\dot{\Omega})}=\left\langle d_{f, h} u, d_{f, h} u_{p}\right\rangle_{L^{2}(\dot{\Omega})}+\left\langle d_{f, h}^{*} u_{p}, d_{f, h}^{*} u_{p}\right\rangle_{L^{2}(\dot{\Omega})}$

Let us now give the proof of the last point (iii), which is a consequence of $(i i)$ together with ideas from 39,54 , since $z_{i}$ is the only generalized critical point of $f$ in $\bar{\Omega}$ for $\Delta_{f, h}^{D,(1)}(\dot{\Omega})$. Let us first prove that for some $c>0$, one has for any $p \in\{0, \ldots, n\}$ and $h$ small enough,

$$
\operatorname{dim} \operatorname{Ran} \pi_{\left[0, c h \frac{3}{2}\right)}\left(\Delta_{f, h}^{M,(p)}(\dot{\Omega})\right)=\delta_{1, p} .
$$

Pick up $u \in D\left(\mathcal{Q}_{f, h}^{M,(p)}(\dot{\Omega})\right)$. From the Green formula 131 and from the fact that $\mathcal{L}_{\nabla f}+\mathcal{L}_{\nabla f}^{*}$ is a $0^{\text {th }}$ order differential operator, there exists $C_{0}>0$ such that for all $u \in D\left(\mathcal{Q}_{f, h}^{M,(p)}(\dot{\Omega})\right)$ and all smooth cut-off function $\chi$ supported in $\dot{\Omega}$ (whose support avoids $\partial \dot{\Omega})$ :

$$
\left\|d_{f, h} \chi u\right\|_{L^{2}(\dot{\Omega})}^{2}+\left\|d_{f, h}^{*} \chi u\right\|_{L^{2}(\dot{\Omega})}^{2} \geq\left(\inf _{\operatorname{supp} \chi}|\nabla f|^{2}-h C_{0}\right)\|\chi u\|^{2} .
$$

Thus, since $f$ has no critical point in $\dot{\Omega}$, there exists some $C>0$ independent of $u \in D\left(\mathcal{Q}_{f, h}^{M,(p)}(\dot{\Omega})\right)$ such that for any smooth cut-off function $\chi$ supported in $\dot{\Omega}$ (whose support avoids $\partial \dot{\Omega})$ and $h$ small enough,

$$
\mathcal{Q}_{f, h}^{M,(p)}(\dot{\Omega})(\chi u) \geq C\|\chi u\|^{2} .
$$

Note in addition that owing to $\partial_{n} f>0$ on $\Gamma_{2} \backslash\left(\overline{\Gamma_{2, i}^{1}} \cap \overline{\Gamma_{2, i}^{2}}\right)$ and $\partial_{n} f<0$ on $\Gamma_{0}$, the boundary terms in the Green formula (131) are non negative, for any smooth cut-off function $\chi$ supported in a neighborhood of any point in $\Gamma_{2} \cup \Gamma_{0}$ (whose support avoids 
some neighborhood of $\Gamma_{1}$ ). Thus, the same considerations show that for $h$ small enough, for such functions $\chi$, taking maybe $C$ smaller, $\forall u \in D\left(\mathcal{Q}_{f, h}^{M,(p)}(\dot{\Omega})\right)$

$$
\mathcal{Q}_{f, h}^{M,(p)}(\dot{\Omega})(\chi u) \geq C\|\chi u\|^{2} .
$$

According to the analysis done in [39, Section 3.4], the same estimate also holds for $\chi$ supported in a sufficiently small neighborhood of some point $x \neq z, x \in \Gamma_{1}$ (whose support avoids a neighborhood of $\left.\{z\} \cup \partial \Gamma_{1}\right)$. This is related to the fact that $\Gamma_{1}$ does not contain any generalized critical point of $f$ in the tangential sense except $z$. Let us now show that such an estimate is also valid near $\partial \Gamma_{1}$. In order to prove it, one recalls that

$$
f=f(z)+f_{+}-f_{-} \text {a.e on } \Omega \text { and }|\nabla f|^{2}=\left|\nabla f_{-}\right|^{2}+\left|\nabla f_{+}\right|^{2} \text { a.e near } \partial \Gamma_{1},
$$

where $f_{ \pm}$are smooth and satisfy the following relations on $B_{z}$ :

$$
f_{+}=f-f(z), f_{-}=0, \partial_{n} f_{+}=0 \text { and } \partial_{n} f_{-}=-\partial_{n} f .
$$

Hence, for any $\chi$ supported in a sufficiently small neighborhood of $\partial \Gamma_{1}$, one deduces from the relation $\mathcal{Q}_{-f_{-}, h}^{M,(p)}(\dot{\Omega})(\chi u) \geq 0$, the Green formula (131), and the fact that $\mathcal{L}_{-\nabla f_{-}}+\mathcal{L}_{-\nabla f_{-}}^{*}$ is a $0^{\text {th }}$ order differential operator, that there exists $C_{1}>0$ independent of $u \in D\left(\mathcal{Q}_{f, h}^{M,(p)}(\dot{\Omega})\right)$ such that:

$$
\begin{aligned}
h\left(\int_{\Gamma_{1}}-\int_{\Gamma_{2}}\right)\langle\chi u, \chi u\rangle_{T_{\sigma}^{*} \dot{\Omega}} \partial_{n} f_{-} d \sigma \geq & -h^{2}\|d \chi u\|_{L^{2}(\dot{\Omega})}^{2}-h^{2}\left\|d^{*} \chi u\right\|_{L^{2}(\dot{\Omega})}^{2} \\
& -\left\|\left|\nabla f_{-}\right| \chi u\right\|_{L^{2}(\dot{\Omega})}^{2}-C_{1} h\|\chi u\|_{L^{2}(\dot{\Omega})}^{2} .
\end{aligned}
$$

Using again the Green formula (131), the relation $f-f(z)=f_{+}-f_{-}$with $\partial_{n} f_{+}=0$ on $\Gamma_{1}$, and the fact that $\mathcal{L}_{\nabla f}+\mathcal{L}_{\nabla f}^{*}$ is a $0^{\text {th }}$ order differential operator then leads to the existence of $C_{2}>0$ independent of $u \in D\left(\mathcal{Q}_{f, h}^{M,(p)}(\dot{\Omega})\right)$ s.t.

$$
\mathcal{Q}_{f, h}^{M,(p)}(\dot{\Omega})(\chi u) \geq\left\|\left|\nabla f_{+}\right| \chi u\right\|_{L^{2}(\dot{\Omega})}^{2}-C_{2} h\|\chi u\|_{L^{2}(\dot{\Omega})}^{2}+h \int_{\Gamma_{2}}\langle\chi u \mid \chi u\rangle_{T_{\sigma}^{*} \dot{\Omega}} \partial_{n} f_{+} d \sigma .
$$

Since $f_{+}$has no critical point around $\partial \Gamma_{1}$ (see $(140)$ ), one has then for $h$ small enough, taking maybe $C$ smaller:

$$
\mathcal{Q}_{f, h}^{M,(p)}(\dot{\Omega})(\chi u) \geq C\|\chi u\|_{L^{2}(\dot{\Omega})}^{2}+h \int_{\Gamma_{2}}\langle\chi u \mid \chi u\rangle_{T_{\sigma}^{*} \dot{\Omega}} \partial_{n} f_{+} d \sigma .
$$

Let us recall that due to our construction of $\Gamma_{2}$ near $\partial \Gamma_{1}$, one has (see 1143 in Proposition 81):

$$
\partial_{n} f_{+}(\sigma)>0 \text { for } \sigma \in \Gamma_{2} \text { sufficiently close to } \partial \Gamma_{1} .
$$

This implies that for $\chi$ supported near $\partial \Gamma_{1}$ with sufficiently small support and $h$ small enough:

$$
\mathcal{Q}_{f, h}^{M,(p)}(\dot{\Omega})(\chi u) \geq C\|\chi u\|_{L^{2}(\dot{\Omega})}^{2} .
$$

Lastly, since $z$ is a generalized critical point with index 1 in the tangential sense, it follows from [39, Proposition 4.3.2] that for $\chi$ supported in a neighborhood of $z$ and $h$ 
small enough, the spectrum of the Friedrichs extension associated with the quadratic form

$$
\left\{v \in \Lambda^{p} H^{1}(\operatorname{supp} \chi) ;\left.\mathbf{t} v\right|_{\Gamma_{1}}=\left.v\right|_{\partial \operatorname{supp} \chi \backslash \bar{\Gamma}_{1}}=0\right\} \ni v \mapsto\left\|d_{f, h} v\right\|_{L^{2}}^{2}+\left\|d_{f, h}^{*} v\right\|_{L^{2}}^{2}
$$

does not meet $\left[0, h^{\frac{3}{2}}\right)$ if $p \neq 1$, and consists of exactly one eigenvalue in $\left[0, h^{\frac{3}{2}}\right)$ which is actually exponentially small - i.e. of the size $O\left(e^{-\frac{C_{3}}{h}}\right)-$ if $p=1$. Denote by $\psi_{1} \in \Lambda^{1} H^{1}(\operatorname{supp} \chi)$ some normalized eigenvalue associated with this exponentially small eigenvalue.

Using the IMS localization formula (see for example [14, Theorem 3.2])

$$
\begin{aligned}
& \forall\left(\chi_{k}\right)_{k \in\{1, \ldots, K\}} \in\left(C^{\infty}(\dot{\dot{\Omega}})\right)^{K} \text { s.t. } \sum_{k=1}^{K} \chi_{k}^{2}=1 \\
& \mathcal{Q}_{f, h}^{M,(p)}(\dot{\Omega})(u)=\sum_{k=1}^{K}\left(\mathcal{Q}_{f, h}^{M,(p)}(\dot{\Omega})\left(\chi_{k} u\right)-h^{2}\left\|\left|\nabla \chi_{k}\right| u\right\|_{L^{2}(\dot{\Omega})}^{2}\right),
\end{aligned}
$$

the previous analysis then shows that choosing $\chi_{1} \in C^{\infty}(\overline{\dot{\Omega}})$ supported in a neighborhood of $z$ with $\chi_{1}=1$ near $z$, one gets for some $C, C^{\prime}>0$ independent of $u \in$ $D\left(\mathcal{Q}_{f, h}^{M,(p)}(\dot{\Omega})\right)$ and $h$ small enough:

$$
\mathcal{Q}_{f, h}^{M,(p)}(\dot{\Omega})(u) \geq \mathcal{Q}_{f, h}^{M,(p)}(\dot{\Omega})\left(\chi_{1} u\right)+C\left\|\left(1-\chi_{1}^{2}\right)^{\frac{1}{2}} u\right\|_{L^{2}(\dot{\Omega})}^{2}-C^{\prime} h^{2}\|u\|_{L^{2}(\dot{\Omega})}^{2} .
$$

If $p \neq 1$, one deduces immediately from 152 that for $h$ small enough,

$$
\mathcal{Q}_{f, h}^{M,(p)}(\dot{\Omega})(u) \geq h^{\frac{3}{2}}\left\|\chi_{1} u\right\|_{L^{2}(\dot{\Omega})}^{2}+C\left\|\left(1-\chi_{1}^{2}\right)^{\frac{1}{2}} u\right\|_{L^{2}(\dot{\Omega})}^{2}-C^{\prime} h^{2}\|u\|_{L^{2}(\dot{\Omega})}^{2},
$$

and then that for some $c>0$ and $h$ small enough:

$$
\mathcal{Q}_{f, h}^{M,(p)}(\dot{\Omega})(u) \geq \operatorname{ch}^{\frac{3}{2}}\|u\|_{L^{2}(\dot{\Omega})}^{2} .
$$

If $p=1$, one obtains from 152 the same conclusion for any $u$ such that $\int_{\dot{\Omega}} u \chi_{1} \psi_{1}=0$ and therefore $\Delta_{f, h}^{M,(p)}(\dot{\Omega})$ has no eigenvalue in $\left[0, c h^{\frac{3}{2}}\right)$ if $p \neq 1$ and at most one if $p=1$. To end up the proof, it is sufficient to remark that $\tilde{\psi}_{1}$, the extension of $\psi_{1}$ to $\dot{\Omega}$ by 0 outside supp $\chi_{1}$, belongs to $D\left(\mathcal{Q}_{f, h}^{M,(1)}(\dot{\Omega})\right)$ and satisfies for $h$ small:

$$
\mathcal{Q}_{f, h}^{M,(1)}(\dot{\Omega})\left(\tilde{\psi}_{1}\right)=\left\|d_{f, h} \psi_{1}\right\|_{L^{2}(\dot{\Omega})}+\left\|d_{f, h}^{*} \psi_{1}\right\|_{L^{2}(\dot{\Omega})}=O\left(e^{-\frac{C_{3}}{h}}\right)<c h^{\frac{3}{2}} .
$$

This proves that there exists $c>0$ and $h_{0}>0$ such that for all $p \in\{0, \ldots, n\}$ and $h \in\left(0, h_{0}\right)$,

$$
\operatorname{dim} \operatorname{Ran} \pi_{\left[0, \operatorname{ch}^{\frac{3}{2}}\right)}\left(\Delta_{f, h}^{M,(p)}\left(\dot{\Omega}_{i}\right)\right)=\delta_{1, p} .
$$

Then, the fact that there exists $c>0$ and $h_{0}>0$ such that for all $h \in\left(0, h_{0}\right)$,

$$
\operatorname{Sp}\left(\Delta_{f, h}^{M,(1)}\left(\dot{\Omega}_{i}\right)\right) \cap\left[0, \operatorname{ch}^{\frac{3}{2}}\right)=\{0\},
$$

is a direct consequence of item (ii) in Proposition 83 together with 153 . This concludes the proof of Proposition 83 . 
Following Proposition 83 , let us introduce an $L^{2}$-normalized eigenform $u_{h, i}^{(1)}$ of $\Delta_{f, h}^{M,(1)}\left(\dot{\Omega}_{i}\right)$ associated with the eigenvalue 0 :

$$
\Delta_{f, h}^{M,(1)}\left(\dot{\Omega}_{i}\right) u_{h, i}^{(1)}=0 \text { in } \dot{\Omega}_{i} \text { and }\left\|u_{h, i}^{(1)}\right\|_{L^{2}\left(\dot{\Omega}_{i}\right)}=1 .
$$

Using standard elliptic regularity results, one can check that $u_{h, i}^{(1)}$ is actually in $C^{\infty}\left(\dot{\Omega}_{i}\right)$ and is smooth in a neighborhood of any regular point of $\partial \dot{\Omega}_{i}$. Notice that thanks to item (iii) in Proposition 83. $u_{h, i}^{(1)}$ is unique up to a multiplication by \pm 1 : this multiplicative constant will be fixed in Proposition 90 below. The quasi-mode $\tilde{\phi}_{i}$ will be built using a suitable truncation of $u_{h, i}^{(1)}$.

\subsection{Definition of the quasi-modes}

Troughout this section, one assumes [H1], [H2] and [H3]. In this section, we construct the function $\tilde{u}$ and a family of 1 -forms $\left(\tilde{\phi}_{i}\right)_{i=1, \ldots, n}$ which will satisfy the estimates stated in Section 2.2.2. For each $i \in\{1, \ldots, n\}$, the 1 -form $\tilde{\phi}_{i}$ will be constructed by a suitable truncation of an eigenfunction $u_{h, i}$ associated with the eigenvalue 0 of the mixed Witten Laplacian attached with $z_{i} \in\left\{z_{1}, \ldots, z_{n}\right\}$, as defined in Section 4.1.3.

We recall that $\Sigma_{i}$ is an open set included in $\partial \Omega$ containing $z_{i}$ which is such that $\bar{\Sigma}_{i} \subset B_{z_{i}}$ (see Definition 9).

\subsubsection{Definition of the quasi-mode $\tilde{u}$}

Definition 84. Let us consider the global minimum $x_{0}$ introduced in the hypothesis [H2]. Let $\chi \in C_{c}^{\infty}(\Omega)$ such that $\{x \in \Omega \mid \chi(x)=1\}$ is a neighborhood of $x_{0}$ and such that $0 \leq \chi \leq 1$ (in particular $\chi\left(x_{0}\right)=1$ ). The quasi-mode $\tilde{u}$ is defined by

$$
\tilde{u}:=\frac{\chi}{\sqrt{\int_{\Omega} \chi^{2} e^{-\frac{2 f}{h}}}} .
$$

The function $\tilde{u}$ belongs to $C_{c}^{\infty}(\Omega)$ and therefore $\tilde{u} \in H_{0}^{1}\left(e^{-\frac{2}{h} f(x)} d x\right)$. The function $\chi$ will be chosen such that $\operatorname{supp}(|\nabla \chi|)$ is as close as needed to $\partial \Omega$, as will be made precise in Section 4.5.

Let us first prove that $\tilde{u}$ satisfies item $2(b)$ in Proposition 25.

Lemma 85. Let us assume that the hypotheses [H1] and [H2] hold. Then for any $\delta>0$, there exist $h_{0}>0, C>0$, and there exists $\chi \in C_{c}^{\infty}(\Omega)$ such that the set $\{x \in \Omega \mid \chi(x)=1\}$ is a neighborhood of $x_{0}, 0 \leq \chi \leq 1$ and for all $h \in\left(0, h_{0}\right)$

$$
\int_{\Omega}|\nabla \tilde{u}|^{2} e^{-\frac{2 f}{h}} \leq C h^{-\frac{d}{2}} e^{-2 \frac{\left(f\left(z_{1}\right)-f\left(x_{0}\right)\right)-\delta}{h}}
$$

where $\tilde{u}$ is defined in Definition 84.

Proof. There exists a constant $C$ such that

$$
\int_{\Omega}|\nabla \tilde{u}(x)|^{2} e^{-\frac{2 f(x)}{h}} d x \leq C \frac{\int_{\operatorname{supp} \nabla \chi} e^{-2 \frac{f(x)}{h}} d x}{\int_{\Omega} \chi^{2}(y) e^{-2 \frac{f(y)}{h}} d y} .
$$


Since supp $\nabla \chi$ can be chosen arbitrarly close to $\partial \Omega$ and since $z_{1}$ is the minimum of $V$ on $\partial \Omega$, by continuity of $f$, for any $\delta>0$ there exists $\chi \in C^{\infty}(\Omega)$ such that $\{x \in \Omega \mid \chi(x)=1\}$ is a neighborhood of $x_{0}, 0 \leq \chi \leq 1$ and

$$
\int_{\operatorname{supp} \nabla \chi} e^{-2 \frac{f(x)}{h}} d x \leq C e^{-2 \frac{f\left(z_{1}\right)+2 \delta}{h}} .
$$

Moreover, since $x_{0}$ is the global minimum of $f$ in $\Omega$, one gets, using Laplace's method

$$
\int_{\Omega} \chi^{2}(y) e^{-2 \frac{f(y)}{h}} d y=\frac{(\pi h)^{\frac{d}{2}}}{\sqrt{\operatorname{det} \operatorname{Hess} f\left(x_{0}\right)}} e^{-2 \frac{f\left(x_{0}\right)}{h}}(1+O(h)) .
$$

This yields the desired estimate.

Notice that item 2(b) in Proposition 25 is a direct consequence of Lemma 85.

\subsubsection{Definition of the quasi-mode $\tilde{\phi}_{i}$ attached to $z_{i}$}

Let $z_{i}$ be a local minimum of $\left.f\right|_{\partial \Omega}$. Let us recall that $\Sigma_{i}$ is an open subset of $\partial \Omega$ such that $z_{i} \in \Sigma_{i}$ and $\overline{\Sigma_{i}} \subset B_{z_{i}}$. Let $\mathcal{S}_{M, i}:=\left\{\dot{\Omega}_{i}, \Gamma_{0}, \Gamma_{1, i}, \Gamma_{2, i}, V_{\Gamma_{1, i}}\right\}$ be an ensemble of sets associated with $z_{i}$, see Definition 82 . Thanks to Propositions 80 and 81 , the set $\Gamma_{1, i}$ can be taken such that

$$
\bar{\Sigma}_{i} \subset \Gamma_{1, i}
$$

We recall that Section 4.1 was dedicated to the construction of a domain $\dot{\Omega}_{i} \subset \Omega$ and a mixed Witten Laplacian $\Delta_{f, h}^{M,(1)}\left(\dot{\Omega}_{i}\right)$ (see 127) ) associated with this ensemble of sets $\mathcal{S}_{M, i}$. Proposition 83 gives the spectral properties of the operator $\Delta_{f, h}^{M}\left(\dot{\Omega}_{i}\right)$. In the following, we consider a normalized eigenform $u_{h, i}^{(1)} \in D\left(\Delta_{f, h}^{M,(1)}\left(\dot{\Omega}_{i}\right)\right)$ associated with the first eigenvalue 0 , i.e. such that $(154)$ holds

The quasi-mode $\tilde{\phi}_{i}$ is defined as the following truncation of $u_{h, i}^{(1)}$.

Definition 86. Let us assume that the hypotheses [H1], [H2] and [H3] hold. Let $\chi_{i} \in C^{\infty}(\bar{\Omega})$ be such that:

1. $\chi_{i} \in C_{c}^{\infty}\left(\dot{\Omega}_{i} \cup \Gamma_{1, i}\right)$ (and thus $\chi_{i}=0$ on a neighborhood of $\Gamma_{2, i} \cup \Gamma_{0}$ and on a neighborhood of $\left.\partial \Omega \backslash \Gamma_{1, i}\right)$,

2. $\chi_{i}=1$ on a neighborhood of $\bar{\Sigma}_{i}$ in $\overline{\dot{\Omega}_{i}}$,

3. $0 \leq \chi_{i} \leq 1$.

One defines $\mathcal{V}_{i}:=\left\{x \in \Omega \mid \chi_{i}(x)=1\right\}$. The quasi-mode $\tilde{\phi}_{i}$ is defined on $\Omega$ by:

$$
\tilde{\phi}_{i}:=\frac{\chi_{i} u_{h, i}^{(1)}}{\sqrt{\int_{\Omega}\left|\chi_{i}(x) u_{h, i}^{(1)}(x)\right|^{2} d x}} .
$$

The support of $\chi_{i}$ on $\partial \Omega$ is represented on Figure 14 and the support of $\chi_{i}$ in $\Omega$ is represented in Figure 15. The sets $\Gamma_{1, i}, \Gamma_{2, i}$ and, the function $\chi_{i}$ will be chosen such that $\operatorname{supp}\left(\left|\nabla \chi_{i}\right|\right)$ is as close as needed from $B_{z_{i}}^{c} \subset \partial \Omega$ or from $x_{0}$, as will be made precise 


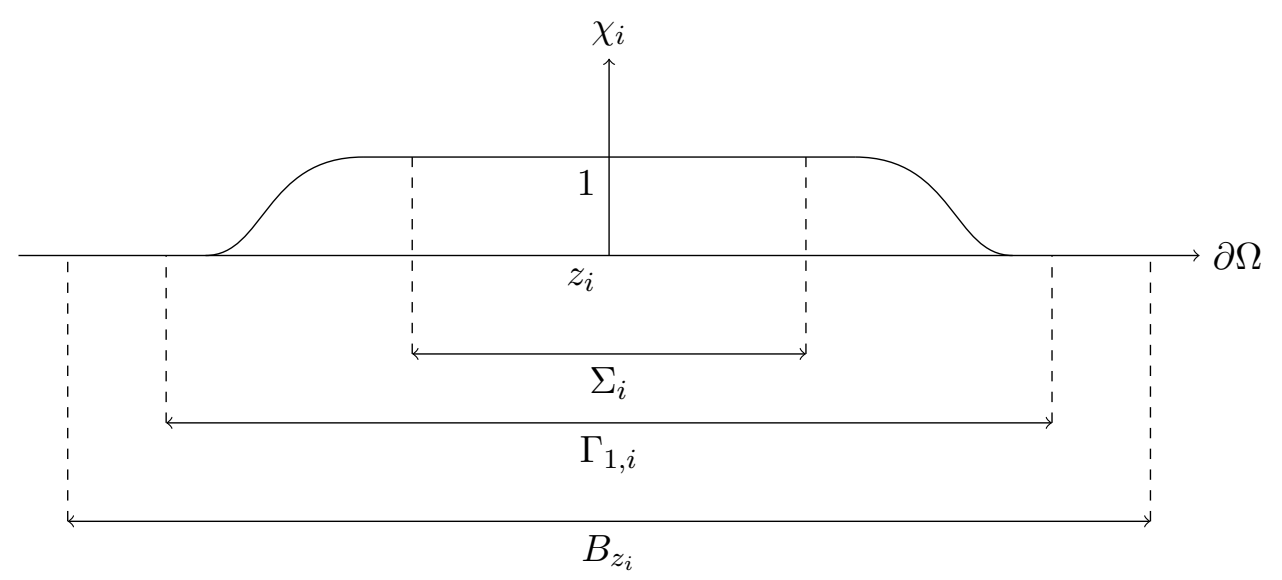

Figure 14: The support of $\chi_{i}$ on $\partial \Omega$.

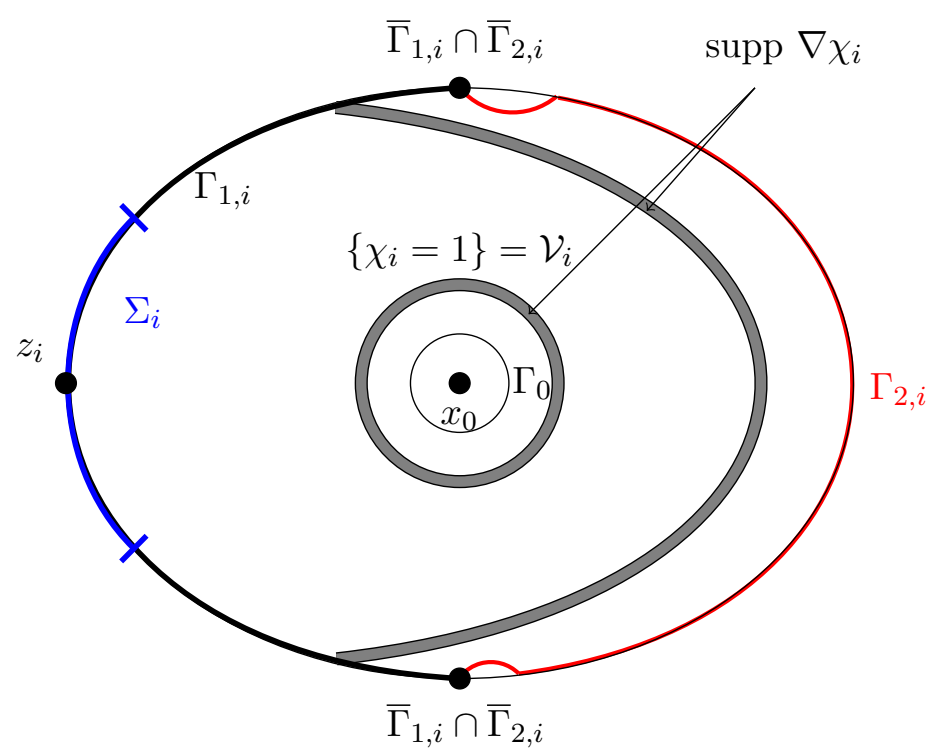

Figure 15: The set $\mathcal{V}_{i}=\left\{x \in \Omega \mid \chi_{i}(x)=1\right\}$ and, in gray, the support of $\nabla \chi_{i}$.

at the end of Section 4.5. This is possible thanks to item 7 in Proposition 81. Using Lemma 73 and the fact that $\mathbf{t} \chi_{i} u_{h, i}^{(1)}=0$ on $\partial \Omega$, one easily shows that

$$
\tilde{\phi}_{i} \in \Lambda^{1} H_{T}^{1}(\Omega) .
$$

Using now the regularity of $u_{h, i}^{(1)}$, one can check that $\tilde{\phi}_{i}$ is actually a $C_{c}^{\infty}\left(\Omega \cup \Gamma_{1, i}\right)$ function.

We will show in Section 4.5 that the family of forms $\left(\tilde{u}, \tilde{\phi}_{1}, \ldots, \tilde{\phi}_{n}\right)$ satisfy the estimates stated in Sections 2.2.1 and 2.2.2. This requires some preliminary results on the eigenforms $\left(u_{h, i}^{(1)}\right)_{i \in\{1, \ldots . n\}}$ that are provided in Section 4.3 and 4.4 


\subsection{Agmon estimates on $u_{h, i}^{(1)}$}

Throughout this section, one assumes [H1], [H2] and [H3]. In all this section, we consider, for a fixed critical point $z_{i}$, an ensemble of sets $\mathcal{S}_{M, i}$ associated with $z_{i}$ (see Definition 82 and an $L^{2}$-normalized eigenform $u_{h, i}^{(1)}$ of $\Delta_{f, h}^{M,(1)}\left(\dot{\Omega}_{i}\right)$ associated with the eingevalue 0 , as introduced at the end of Section 4.1 .3 .

The aim of this section is to prove the following proposition.

Proposition 87. Let us assume that the hypotheses [H1], [H2] and [H3] hold. Any $L^{2}$-normalized eigenform $u_{h, i}^{(1)}$ of $\Delta_{f, h}^{M,(1)}\left(\dot{\Omega}_{i}\right)$ associated with the eigenvalue 0 satisfies:

$$
\exists N \in \mathbb{N},\left\|e^{\frac{\Psi_{i}}{h}} u_{h, i}^{(1)}\right\|_{L^{2}\left(\dot{\Omega}_{i}\right)}+\left\|d\left(e^{\frac{\Psi_{i}}{h}} u_{h, i}^{(1)}\right)\right\|_{L^{2}\left(\dot{\Omega}_{i}\right)}+\left\|d^{*}\left(e^{\frac{\Psi_{i}}{h}} u_{h, i}^{(1)}\right)\right\|_{L^{2}\left(\dot{\Omega}_{i}\right)}=O\left(h^{-N}\right)
$$

where, we recall, $\Psi_{i}(x)=d_{a}\left(x, z_{i}\right)$ (see Definition 78$)$.

For the ease of notation, we drop the subscript $i$ in the remaining of this section.

The proof is inspired by the first part of the proof of [39, Proposition 4.3.2] where the authors consider a Witten Laplacian with mixed tangential - full Dirichlet boundary conditions in a local system of coordinates in a neighborhood of $z$. The proof actually only requires that $u_{h}^{(1)}$ is an eigenform of $\Delta_{f, h}^{M,(1)}(\dot{\Omega})$ associated with an eigenvalue $\lambda_{h}=$ $O(h)$. It crucially relies on the following Agmon-type energy equality.

Lemma 88. Let us assume that the hypotheses [H1], [H2] and [H3] hold. Let $\varphi$ be a real-valued Lipschitz function on $\bar{\Omega}$. Then, for any $u \in D\left(\mathcal{Q}_{f, h}^{M,(1)}(\dot{\Omega})\right)$, one has:

$$
\begin{aligned}
\mathcal{Q}_{f, h}^{M,(1)}(\dot{\Omega})\left(u, e^{2 \frac{\varphi}{h}} u\right)= & h^{2}\left\|d e^{\frac{\varphi}{h}} u\right\|_{L^{2}(\dot{\Omega})}^{2}+h^{2}\left\|d^{*} e^{\frac{\varphi}{h}} u\right\|_{L^{2}(\dot{\Omega})}^{2} \\
& +\left\langle\left(|\nabla f|^{2}-|\nabla \varphi|^{2}+h \mathcal{L}_{\nabla f}+h \mathcal{L}_{\nabla f}^{*}\right) e^{\frac{\varphi}{h}} u, e^{\frac{\varphi}{h}} u\right\rangle_{L^{2}(\dot{\Omega})} \\
& +h\left(-\int_{\Gamma_{0} \cup \Gamma_{1}}+\int_{\Gamma_{2}}\right)\langle u, u\rangle_{T_{\sigma}^{*} \dot{\Omega}} e^{\frac{2}{h} \varphi} \partial_{n} f d \sigma .
\end{aligned}
$$

Moreover, when $u \in D\left(\Delta_{f, h}^{M,(1)}(\dot{\Omega})\right)$, the left-hand side equals $\left\langle e^{2 \frac{\varphi}{h}} \Delta_{f, h}^{M,(1)}(\dot{\Omega}) u, u\right\rangle_{L^{2}(\dot{\Omega})}$.

Proof. This result is standard for manifolds without boundary or for bounded manifolds and quadratic forms with full normal or tangential boundary conditions (see e.g. 25 , $39,54)$. We extend it here to our setting.

Note first that $u \in D\left(\mathcal{Q}_{f, h}^{M,(1)}(\dot{\Omega})\right)$ implies $e^{2 \frac{\varphi}{h}} u \in D\left(\mathcal{Q}_{f, h}^{M,(1)}(\dot{\Omega})\right)$, since for $u \in$ $D\left(\mathcal{Q}_{f, h}^{M,(1)}(\dot{\Omega})\right), n^{b} \wedge e^{2 \frac{\varphi}{h}} u=e^{2 \frac{\varphi}{h}} n^{b} \wedge u$ and $\mathbf{i}_{n} e^{2 \frac{\varphi}{h}} u=e^{2 \frac{\varphi}{h}} \mathbf{i}_{n} u$. One then gets by straightforward computations:

$$
\begin{aligned}
\mathcal{Q}_{f, h}^{M,(1)}(\dot{\Omega})\left(u, e^{2 \frac{\varphi}{h}} u\right)= & \left\langle d_{f, h} u, d_{f, h}\left(e^{2 \frac{\varphi}{h}} u\right)\right\rangle+\left\langle d_{f, h}^{*} u, d_{f, h}^{*}\left(e^{2 \frac{\varphi}{h}} u\right)\right\rangle \\
= & \left\langle e^{\frac{\varphi}{h}} d_{f, h} u, d_{f, h}\left(e^{\frac{\varphi}{h}} u\right)\right\rangle+\left\langle e^{\frac{\varphi}{h}} d_{f, h} u, d \varphi \wedge\left(e^{\frac{\varphi}{h}} u\right)\right\rangle \\
& +\left\langle e^{\frac{\varphi}{h}} d_{f, h}^{*} u, d_{f, h}^{*}\left(e^{\frac{\varphi}{h}} u\right)\right\rangle-\left\langle e^{\frac{\varphi}{h}} d_{f, h}^{*} u, \mathbf{i}_{\nabla \varphi}\left(e^{\frac{\varphi}{h}} u\right)\right\rangle \\
= & \left\langle d_{f, h}\left(e^{\frac{\varphi}{h}} u\right), d \varphi \wedge\left(e^{\frac{\varphi}{h}} u\right)\right\rangle-\left\langle d \varphi \wedge\left(e^{\frac{\varphi}{h}} u\right), d_{f, h}\left(e^{\frac{\varphi}{h}} u\right)\right\rangle \\
& +\left\|d_{f, h}\left(e^{\frac{\varphi}{h}} u\right)\right\|^{2}-\left\|d \varphi \wedge\left(e^{\frac{\varphi}{h}} u\right)\right\|^{2}+\left\|d_{f, h}^{*}\left(e^{\frac{\varphi}{h}} u\right)\right\|^{2}-\left\|\mathbf{i}_{\nabla \varphi} e^{\frac{\varphi}{h}} u\right\|^{2} \\
& +\left\langle\mathbf{i}_{\nabla \varphi}\left(e^{\frac{\varphi}{h}} u\right), d_{f, h}^{*}\left(e^{\frac{\varphi}{h}} u\right)\right\rangle-\left\langle d_{f, h}^{*}\left(e^{\frac{\varphi}{h}} u\right), \mathbf{i}_{\nabla \varphi}\left(e^{\frac{\varphi}{h}} u\right)\right\rangle .
\end{aligned}
$$


Let us set $\tilde{u}:=e^{\frac{\varphi}{h}} u \in D\left(Q_{f, h}^{M,(1)}(\dot{\Omega})\right)$. The formulas stated in 132 lead to:

$$
\begin{aligned}
\mathcal{Q}_{f, h}^{M,(1)}(\dot{\Omega})\left(u, e^{2 \frac{\varphi}{h}} u\right)= & \mathcal{Q}_{f, h}^{M,(1)}(\dot{\Omega})(\tilde{u})-\left\langle|\nabla \varphi|^{2} \tilde{u}, \tilde{u}\right\rangle-\left\langle d \varphi \wedge \tilde{u}, d_{f, h} \tilde{u}\right\rangle \\
& +\left\langle d_{f, h} \tilde{u}, d \varphi \wedge \tilde{u}\right\rangle+\left\langle\mathbf{i}_{\nabla \varphi} \tilde{u}, d_{f, h}^{*} \tilde{u}\right\rangle-\left\langle d_{f, h}^{*} \tilde{u}, \mathbf{i}_{\nabla \varphi} \tilde{u}\right\rangle
\end{aligned}
$$

and hence to

$$
\mathcal{Q}_{f, h}^{M,(1)}(\dot{\Omega})\left(u, e^{2 \frac{\varphi}{h}} u\right)=\mathcal{Q}_{f, h}^{M,(1)}(\dot{\Omega})(\tilde{u})-\left\langle|\nabla \varphi|^{2} \tilde{u}, \tilde{u}\right\rangle .
$$

One concludes by applying Lemma 76 .

We are now in position to prove Proposition 87.

Proof. (of Proposition 87)

Following the proof of [39, Proposition 4.3.2], one proves the result in two steps. First, the Agmon estimate along $\Gamma_{1} \subset \partial \Omega$ is proven by applying Lemma 88 with a function $\varphi$ close to $f_{+}$(recall that on $\Gamma_{1}, \Psi=f_{+}$). The Agmon estimate in $\dot{\Omega}$ is then obtained using again Lemma 88 with $\varphi$ close to $\Psi$, and the Agmon estimate along $\Gamma_{1}$.

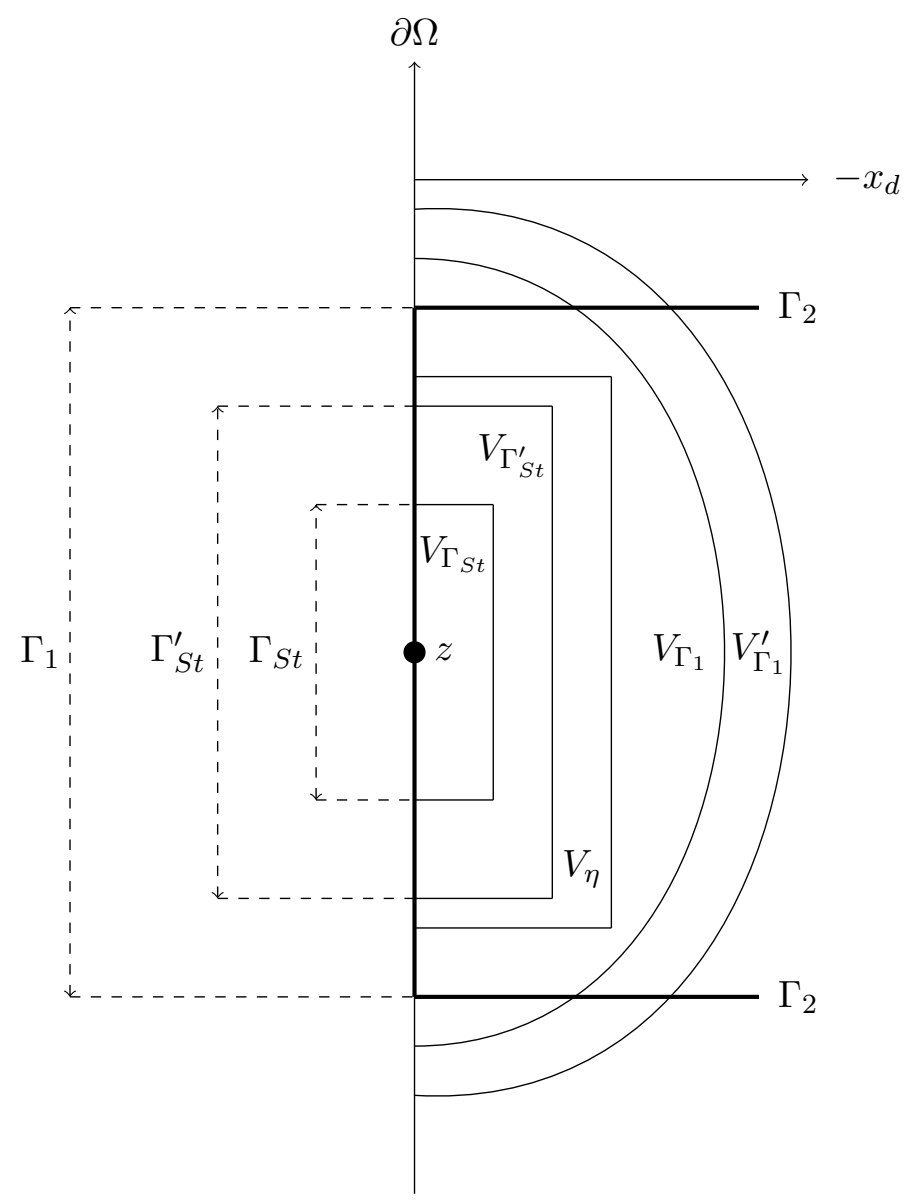

Figure 16: Neighborhoods of $z$.

In order to separate the analysis along $\Gamma_{1}$ and elsewhere, one introduces two smooth cut-off functions $\chi_{0}$ and $\chi_{1}$ on $\bar{\Omega}$ such that:

$$
\chi_{1}:=\sqrt{1-\chi_{0}^{2}}, \quad \chi_{0}=1 \text { on } \overline{V_{\Gamma_{1}}} \text { with } \operatorname{supp} \chi_{0} \subset V_{\Gamma_{1}}^{\prime},
$$


for a set $V_{\Gamma_{1}}^{\prime} \subset \bar{\Omega}$ such that for some $\varepsilon>0$, (see Figure 16 )

(i) $\left(V_{\Gamma_{1}}+B(0, \varepsilon)\right) \cap \bar{\Omega} \subset V_{\Gamma_{1}}^{\prime}$,

(ii) $\Gamma_{1}^{\prime}:=V_{\Gamma_{1}}^{\prime} \cap \partial \Omega$ is smooth and $\left(\Gamma_{1}+B(0, \varepsilon)\right) \cap \partial \Omega \subset \Gamma_{1}^{\prime}$ and $\left(\Gamma_{1}^{\prime}+B(0, \varepsilon)\right) \cap \partial \Omega \subset B_{z}$,

(iii) $\Psi=d_{a}(z, \cdot)$ is a smooth solution to the following eikonal equation in $V_{\Gamma_{1}}^{\prime}$ (see Proposition 71):

$$
\left\{\begin{array}{c}
|\nabla \Psi|^{2}=|\nabla f|^{2} \text { in } V_{\Gamma_{1}}^{\prime} \\
\Psi=f-f(z) \text { on } \Gamma_{1}^{\prime} \\
\partial_{n} \Psi=-\partial_{n} f \text { on } \Gamma_{1}^{\prime} .
\end{array}\right.
$$

It is possible to choose $V_{\Gamma_{1}}^{\prime}$ such that all the properties stated previously on $V_{\Gamma_{1}}$ also hold on $V_{\Gamma_{1}}^{\prime}$ (in particular (138), 139) and the properties stated in Proposition 81). We recall that one has by 777$)$ :

$$
|\nabla \Psi|^{2} \leq|\nabla f|^{2} \quad \text { a.e. in } \Omega
$$

Thus

$$
\left|\nabla f_{ \pm}\right|=\left|\nabla\left(\frac{\Psi \pm(f-f(z))}{2}\right)\right| \leq|\nabla f| \text { a.e. in } \Omega .
$$

Thanks to the relations $f-f(z)=f_{+}-f_{-}$and $\Psi=f_{+}+f_{-}$, together with the equality $|\nabla \Psi|^{2}=|\nabla f|^{2}$ a.e in $V_{\Gamma_{1}}^{\prime}$, one has

$$
\nabla f_{-} \cdot \nabla f_{+}=0 \quad \text { a.e in } V_{\Gamma_{1}}^{\prime},|\nabla \Psi|^{2}=|\nabla f|^{2}=\left|\nabla f_{+}\right|^{2}+\left|\nabla f_{-}\right|^{2} \quad \text { a.e in } V_{\Gamma_{1}}^{\prime} .
$$

Let now $u_{h}^{(1)} \in D\left(\Delta_{f, h}^{M,(1)}(\dot{\Omega})\right)$ satisfy

$$
\Delta_{f, h}^{M,(1)}(\dot{\Omega}) u_{h}^{(1)}=0 \text { and }\left\|u_{h}^{(1)}\right\|_{L^{2}(\dot{\Omega})}=1 .
$$

Step 1: Agmon estimate in $\Gamma_{1}$.

In this part, we are going to prove the estimate 157 with $\Psi$ replaced by $f_{+}$namely:

$$
\left\|e^{\frac{f_{+}}{h}} u_{h}^{(1)}\right\|_{L^{2}(\dot{\Omega})}+\left\|d\left(e^{\frac{f_{+}}{h}} u_{h}^{(1)}\right)\right\|_{L^{2}(\dot{\Omega})}+\left\|d^{*}\left(e^{\frac{f_{+}}{h}} u_{h}^{(1)}\right)\right\|_{L^{2}(\dot{\Omega})}=O\left(h^{-N_{0}}\right)
$$

for some integer $N_{0}$. By the trace result (126), this will give the estimate

$$
\left\|e^{\frac{f-f(z)}{h}} u_{h}^{(1)}\right\|_{L^{2}\left(\Gamma_{1}\right)}=O\left(h^{-N_{0}}\right)
$$

which is the first step to prove (157).

To get these results, the idea is to apply Lemma 88 to a convenient $\varphi$ comparable with $f_{+}$and such that $|\nabla \varphi| \leq\left|\nabla f_{+}\right|$. This kind of estimate is classic and the ideas behind the computations presented below, which follow [39, 54], originate from the article [40] where similar estimates were obtained in the case of manifolds without boundary. The presence of a boundary leads here to some technicalities which can somehow hide the reasoning and we refer for example to 25,35 for a presentation of semi-classical Agmon estimates in manifolds without boundary. We recall from the work [40] that if one just wants to get an error of the size $O\left(e^{\frac{\varepsilon}{h}}\right)$ with $\varepsilon>0$ arbitrarily small, the choice $\varphi=(1-\varepsilon) f_{+}$is sufficient, but it does not yield an error of the size $O\left(h^{-N}\right)$. To get such an error term, a good choice for $\varphi$ is the following. 
Let $\varphi: \dot{\Omega} \rightarrow \mathbb{R}$ be the following Lipschitz function:

$$
\varphi=\left\{\begin{array}{c}
f_{+}-C h \ln \frac{f_{+}}{h} \text { if } f_{+}>C h, \\
f_{+}-C h \ln C \text { if } f_{+} \leq C h,
\end{array}\right.
$$

for some constant $C>1$ that will be fixed at the end of this step. Define the level sets:

$$
\Omega_{-}=\left\{x \in \dot{\Omega} \text { s.t. } f_{+}(x) \leq C h\right\} \quad \text { and } \quad \Omega_{+}=\dot{\Omega} \backslash \Omega_{-} .
$$

Then $\nabla \varphi=\nabla f_{+}$a.e. in $\Omega_{-}$and

$$
\nabla \varphi=\nabla f_{+}\left(1-\frac{C h}{f_{+}}\right) \quad \text { a.e. in } \Omega_{+} .
$$

This implies in particular the two following inequalities valid a.e. on $\Omega_{+}$and that will be used in the sequel:

$$
\begin{gathered}
\left|\nabla f_{+}\right|^{2}-|\nabla \varphi|^{2}=\left|\nabla f_{+}\right|^{2}\left(\frac{2 C h}{f_{+}}-\frac{C^{2} h^{2}}{f_{+}^{2}}\right) \geq C h \frac{\left|\nabla f_{+}\right|^{2}}{f_{+}} \text {on } \Omega_{+} \\
|\nabla f|^{2}-|\nabla \varphi|^{2} \geq|\nabla f|^{2}-\left|\nabla f_{+}\right|^{2}\left(1-\frac{C h}{f_{+}}\right) \geq C h \frac{|\nabla f|^{2}}{f_{+}} \text {on } \Omega_{+} .
\end{gathered}
$$

The last inequality in 161$)$ is a consequence of the inequality $\left|\nabla f_{+}\right|^{2} \leq|\nabla f|^{2}$. This implies in particular that $|\nabla \varphi| \leq|\nabla f|$ a.e. in $\dot{\Omega}$.

Note lastly that there exists a constant $K>0$ depending on $f_{+}$and $f$, such that

$$
\frac{|\nabla f|^{2}}{f_{+}} \geq K \text { in } \dot{\Omega} \text { and } \frac{\left|\nabla f_{+}\right|^{2}}{f_{+}} \geq K \text { in } V_{\Gamma_{1}}^{\prime}
$$

the last inequality being a consequence of the facts that $f_{+}\left(x^{\prime}, x_{d}\right)=f_{+}\left(x^{\prime}, 0\right)$ and $x^{\prime} \mapsto f\left(x^{\prime}, 0\right)=f(z)+f_{+}\left(x^{\prime}, 0\right)$ is a Morse function with $z$ as only critical point.

Now, using the fact that $\Delta_{f, h}^{M,(1)}(\dot{\Omega}) u_{h}^{(1)}=0$ and the IMS localisation formula 151, one gets

$$
\begin{aligned}
0 & =\mathcal{Q}_{f, h}^{M,(1)}(\dot{\Omega})\left(u_{h}^{(1)}, e^{2 \frac{\varphi}{h}} u_{h}^{(1)}\right) \\
& =\sum_{k \in\{0,1\}}\left[\mathcal{Q}_{f, h}^{M,(1)}(\dot{\Omega})\left(\chi_{k} u_{h}^{(1)}, e^{2 \frac{\varphi}{h}} \chi_{k} u_{h}^{(1)}\right)-h^{2}\left\|\left|\nabla \chi_{k}\right| e^{\frac{\varphi}{h}} u_{h}^{(1)}\right\|_{L^{2}(\dot{\Omega})}^{2}\right]
\end{aligned}
$$

Setting

$$
\tilde{u}_{h}^{(1)}:=e^{\frac{\varphi}{h}} u_{h}^{(1)},
$$

and applying (158) to $\chi_{k} u_{h}^{(1)}, k \in\{0,1\}$, one obtains:

$$
\begin{aligned}
& C_{1} h^{2} \sum_{k \in\{0,1\}}\left\|\chi_{k} \tilde{u}_{h}^{(1)}\right\|_{L^{2}(\dot{\Omega})}^{2}=C_{1} h^{2}\left\|\tilde{u}_{h}^{(1)}\right\|_{L^{2}(\dot{\Omega})}^{2} \\
& \geq \sum_{k \in\{0,1\}}\left[\left\|h d \chi_{k} \tilde{u}_{h}^{(1)}\right\|_{L^{2}(\dot{\Omega})}^{2}+\left\|h d^{*} \chi_{k} \tilde{u}_{h}^{(1)}\right\|_{L^{2}(\dot{\Omega})}^{2}+\left\langle\left(|\nabla f|^{2}-|\nabla \varphi|^{2}\right) \chi_{k} \tilde{u}_{h}^{(1)}, \chi_{k} \tilde{u}_{h}^{(1)}\right\rangle_{L^{2}(\dot{\Omega})}\right. \\
& \left.\quad+h\left\langle\left(\mathcal{L}_{\nabla f}+\mathcal{L}_{\nabla f}^{*}\right) \chi_{k} \tilde{u}_{h}^{(1)}, \chi_{k} \tilde{u}_{h}^{(1)}\right\rangle_{L^{2}(\dot{\Omega})}\right]+h\left(\int_{\Gamma_{2}}-\int_{\Gamma_{1}}\right)\left\langle\chi_{0} \tilde{u}_{h}^{(1)}, \chi_{0} \tilde{u}_{h}^{(1)}\right\rangle \partial_{n} f d \sigma,
\end{aligned}
$$


where $C_{1}=\max \left(\left\|\nabla \chi_{0}\right\|_{\infty}^{2},\left\|\nabla \chi_{1}\right\|_{\infty}^{2}\right)$. Note that one has used that $\chi_{0}=0$ on $\Gamma_{0}, \chi_{1}=0$ on $\Gamma_{1}$ and

$$
\left(-\int_{\Gamma_{0}}+\int_{\Gamma_{2}}\right)\left\langle\chi_{1} \tilde{u}_{h}^{(1)}, \chi_{1} \tilde{u}_{h}^{(1)}\right\rangle \partial_{n} f d \sigma \geq 0
$$

which follows from $\partial_{n} f>0$ on $\Gamma_{2}$ and from $\partial_{n} f<0$ on $\Gamma_{0}$.

Now, since $\mathcal{L}_{\nabla f}+\mathcal{L}_{\nabla f}^{*}$ is a $0^{\text {th }}$ order differential operator, and $\left|\tilde{u}_{h}^{(1)}(x)\right| \leq e^{C}\left|u_{h}^{(1)}(x)\right|$ a.e. on $\Omega_{-}$, one obtains that for some constants $C_{2}$ (independent of $C$ ) and $C_{3}(C)$ depending on $C$,

$$
\begin{aligned}
C_{3}(C) h \geq & \sum_{k \in\{0,1\}}\left[\left\|h d \chi_{k} \tilde{u}_{h}^{(1)}\right\|_{L^{2}(\dot{\Omega})}^{2}+\left\|h d^{*} \chi_{k} \tilde{u}_{h}^{(1)}\right\|_{L^{2}(\dot{\Omega})}^{2}+\left\langle\left(|\nabla f|^{2}-|\nabla \varphi|^{2}\right) \chi_{k} \tilde{u}_{h}^{(1)}, \chi_{k} \tilde{u}_{h}^{(1)}\right\rangle_{L^{2}(\dot{\Omega})}\right. \\
& \left.-C_{2} h\left\|\chi_{k} \tilde{u}_{h}^{(1)}\right\|_{L^{2}\left(\Omega_{+}\right)}^{2}\right]+h\left(\int_{\Gamma_{2}}-\int_{\Gamma_{1}}\right)\left\langle\chi_{0} \tilde{u}_{h}^{(1)}, \chi_{0} \tilde{u}_{h}^{(1)}\right\rangle \partial_{n} f d \sigma .
\end{aligned}
$$

Let us first consider the case $k=1$. Using $|\nabla \varphi| \leq|\nabla f|$ and (161)-162), one gets:

$$
\begin{aligned}
& \left\langle\left(|\nabla f|^{2}-|\nabla \varphi|^{2}\right) \chi_{1} \tilde{u}_{h}^{(1)}, \chi_{1} \tilde{u}_{h}^{(1)}\right\rangle_{L^{2}(\dot{\Omega})}-C_{2} h\left\|\chi_{1} \tilde{u}_{h}^{(1)}\right\|_{L^{2}\left(\Omega_{+}\right)}^{2} \\
& \geq\left\langle\left(|\nabla f|^{2}-|\nabla \varphi|^{2}\right) \chi_{1} \tilde{u}_{h}^{(1)}, \chi_{1} \tilde{u}_{h}^{(1)}\right\rangle_{L^{2}\left(\Omega_{+}\right)}-C_{2} h\left\|\chi_{1} \tilde{u}_{h}^{(1)}\right\|_{L^{2}\left(\Omega_{+}\right)}^{2} \\
& \geq\left\langle\left(C h \frac{|\nabla f|^{2}}{f_{+}}-C_{2} h\right) \chi_{1} \tilde{u}_{h}^{(1)}, \chi_{1} \tilde{u}_{h}^{(1)}\right\rangle_{L^{2}\left(\Omega_{+}\right)} \\
& \geq\left(K C-C_{2}\right) h\left\|\chi_{1} \tilde{u}_{h}^{(1)}\right\|_{L^{2}\left(\Omega_{+}\right)}^{2}
\end{aligned}
$$

Let us then consider the case $k=0$. In this case, one deduces from $\operatorname{supp} \chi_{0} \subset V_{\Gamma_{1}}^{\prime}$ where $|\nabla f|^{2}=\left|\nabla f_{+}\right|^{2}+\left|\nabla f_{-}\right|^{2}$, from $|\nabla \varphi|^{2}=\left|\nabla f_{+}\right|^{2}$ on $\Omega_{-}$, and from 160-162 the inequality:

$$
\begin{aligned}
\left\langle\left(|\nabla f|^{2}-|\nabla \varphi|^{2}\right) \chi_{0} \tilde{u}_{h}^{(1)}, \chi_{0} \tilde{u}_{h}^{(1)}\right\rangle_{L^{2}(\dot{\Omega})}-C_{2} h\left\|\chi_{0} \tilde{u}_{h}^{(1)}\right\|_{L^{2}\left(\Omega_{+}\right)}^{2} \\
=\left\|\left|\nabla f_{-}\right| \chi_{0} \tilde{u}_{h}^{(1)}\right\|_{L^{2}(\dot{\Omega})}^{2}+\left\langle(\underbrace{\left|\nabla f_{+}\right|^{2}-|\nabla \varphi|^{2}}_{=0}) \chi_{0} \tilde{u}_{h}^{(1)}, \chi_{0} \tilde{u}_{h}^{(1)}\right\rangle_{L^{2}\left(\Omega_{-}\right)} \\
\quad+\left\langle\left(\left|\nabla f_{+}\right|^{2}-|\nabla \varphi|^{2}-C_{2} h\right) \chi_{0} \tilde{u}_{h}^{(1)}, \chi_{0} \tilde{u}_{h}^{(1)}\right\rangle_{L^{2}\left(\Omega_{+}\right)} \\
\geq\left\|\left|\nabla f_{-}\right| \chi_{0} \tilde{u}_{h}^{(1)}\right\|_{L^{2}(\dot{\Omega})}^{2}+\left(K C-C_{2}\right) h\left\|\chi_{0} \tilde{u}_{h}^{(1)}\right\|_{L^{2}\left(\Omega_{+}\right)}^{2} \\
\geq\left(1+2 C_{4}(C) h\right)\left\|\left|\nabla f_{-}\right| \chi_{0} \tilde{u}_{h}^{(1)}\right\|_{L^{2}(\dot{\Omega})}^{2}-\left(K C-C_{2}\right) h\left\|\chi_{0} \tilde{u}_{h}^{(1)}\right\|_{L^{2}\left(\Omega_{-}\right)}^{2},
\end{aligned}
$$

where $C_{4}(C):=\frac{K C-C_{2}}{2\left\|\nabla f_{-}\right\|_{L^{\infty}}^{2}\left(V_{\Gamma_{1}}^{\prime}\right)}($ see $\sqrt{138})$ ) and $C$ has been chosen large enough to ensure that $K C-C_{2}>0$.

In order to get a lower bound for the boundary term in (163), one uses the fact that the mixed Witten Laplacian $\Delta_{\hat{f}, \hat{h}}^{M,(1)}(\dot{\Omega})$ associated with $\hat{f}=-\tilde{\chi}_{0} f_{-}$where $\tilde{\chi}_{0} \in$ $C^{\infty}(\bar{\Omega},[0,1]), \tilde{\chi}_{0}=1$ on $\operatorname{supp} \chi_{0}, \operatorname{supp} \tilde{\chi}_{0} \subset\left(V_{\Gamma_{1}}^{\prime}+B(0, \alpha)\right) \cap \bar{\Omega}$ for $\alpha>0$ such that $f_{-}$ is smooth on supp $\tilde{\chi}_{0}$ and $\hat{h}=\frac{h}{1+C_{4}(C) h}$, is nonnegative. Starting from the inequality $\left(1+C_{4}(C) h\right) \mathcal{Q}_{\hat{f}, \hat{h}}^{M,(1)}(\dot{\Omega})\left(\chi_{0} \tilde{u}_{h}^{(1)}, \chi_{0} \tilde{u}_{h}^{(1)}\right) \geq 0$ and then applying Lemma 76 to $\chi_{0} \tilde{u}_{h}^{(1)} \in$ 
$D\left(\mathcal{Q}_{\hat{f}, \hat{h}}^{M,(1)}(\dot{\Omega})\right)$ lead to (since $\chi_{0}=0$ on $\left.\Gamma_{0}\right)$ :

$$
\begin{gathered}
h\left(\int_{\Gamma_{1}}-\int_{\Gamma_{2}}\right)\left\langle\chi_{0} \tilde{u}_{h}^{(1)}, \chi_{0} \tilde{u}_{h}^{(1)}\right\rangle \partial_{n} f_{-} d \sigma \geq-\left(1+C_{4}(C) h\right)\left\|\left|\nabla f_{-}\right| \chi_{0} \tilde{u}_{h}^{(1)}\right\|_{L^{2}(\dot{\Omega})}^{2} \\
-\frac{h^{2}}{1+C_{4}(C) h}\left(\left\|d \chi_{0} \tilde{u}_{h}^{(1)}\right\|_{L^{2}(\dot{\Omega})}^{2}+\left\|d^{*} \chi_{0} \tilde{u}_{h}^{(1)}\right\|_{L^{2}(\dot{\Omega})}^{2}\right)-h C_{5}\left\|\chi_{0} \tilde{u}_{h}^{(1)}\right\|_{L^{2}(\dot{\Omega})}^{2}
\end{gathered}
$$

where $C_{5}$ is some positive constant independent of $C$ (it only depends on $f_{-}$).

Injecting the estimates 164 166 in 163 and using $f=-f_{-}+f_{+}+f(z)$ on $V_{\Gamma_{1}}^{\prime}$ with $\partial_{n} f_{+}=0$ on $\Gamma_{1}$, then leads to:

$$
\begin{aligned}
C_{3} h \geq & \sum_{k \in\{0,1\}} \frac{C_{4} h^{3}}{1+C_{4} h}\left(\left\|d \chi_{k} \tilde{u}_{h}^{(1)}\right\|_{L^{2}(\dot{\Omega})}^{2}+\left\|d^{*} \chi_{k} \tilde{u}_{h}^{(1)}\right\|_{L^{2}(\dot{\Omega})}^{2}\right)+\left(K C-C_{2}\right) h\left\|\chi_{1} \tilde{u}_{h}^{(1)}\right\|_{L^{2}\left(\Omega_{+}\right)}^{2} \\
& +C_{4} h\left\|\left|\nabla f_{-}\right| \chi_{0} \tilde{u}_{h}^{(1)}\right\|_{L^{2}(\dot{\Omega})}^{2}-\left(K C-C_{2}\right) h\left\|\chi_{0} \tilde{u}_{h}^{(1)}\right\|_{L^{2}\left(\Omega_{-}\right)}^{2} \\
& -h C_{5}\left\|\chi_{0} \tilde{u}_{h}^{(1)}\right\|_{L^{2}(\dot{\Omega})}^{2}+h \int_{\Gamma_{2}}\left\langle\chi_{0} u \mid \chi_{0} u\right\rangle_{T_{\sigma}^{*} \dot{\Omega}} \partial_{n} f_{+} d \sigma .
\end{aligned}
$$

In the last computation, one has used that $1 \geq \frac{C_{4} h}{1+C_{4} h}, 1-\frac{1}{1+C_{4} h}=\frac{C_{4} h}{1+C_{4} h}$. It follows moreover from 143 that $\partial_{n} f_{+}>0$ on supp $\chi_{0} \cap \Gamma_{2}$. Then, since $\left|\tilde{u}_{h}^{(1)}(x)\right| \leq e^{C}\left|u_{h}^{(1)}(x)\right|$ a.e. on $\Omega_{-}$, there exists $C_{6}\left(C, C_{2}, K\right)$ such that

$$
\begin{aligned}
C_{6} h \geq & \sum_{k \in\{0,1\}} \frac{C_{4} h^{3}}{1+C_{4}}\left(\left\|d \chi_{k} \tilde{u}_{h}^{(1)}\right\|_{L^{2}(\dot{\Omega})}^{2}+\left\|d^{*} \chi_{k} \tilde{u}_{h}^{(1)}\right\|_{L^{2}(\dot{\Omega})}^{2}\right)+2 C_{4}\left\|\nabla f_{-}\right\|_{L^{\infty}\left(V_{\Gamma_{1}}^{\prime}\right)}^{2} h\left\|\chi_{1} \tilde{u}_{h}^{(1)}\right\|_{L^{2}(\dot{\Omega})}^{2} \\
& +C_{4} h\left\|\left|\nabla f_{-}\right| \chi_{0} \tilde{u}_{h}^{(1)}\right\|_{L^{2}(\dot{\Omega})}^{2}-h C_{5}\left\|\chi_{0} \tilde{u}_{h}^{(1)}\right\|_{L^{2}(\dot{\Omega})}^{2} .
\end{aligned}
$$

One has used that $1+C_{4} \geq 1+C_{4} h$, for $h \leq 1$ and $K C-C_{2}=2 C_{4}\left\|\nabla f_{-}\right\|_{L^{\infty}\left(V_{\Gamma_{1}}^{\prime}\right.}^{2}$. Additionally, since $\left|\nabla f_{-}\right| \geq c>0$ on $\overline{V_{\Gamma_{1}}^{\prime}}$ (see $(138)$ ), $\lim _{C \rightarrow \infty} C_{4}(C)=+\infty$ and $C_{5}$ is independent of $C$, one can then choose $C$ such that $c^{2} C_{4}-C_{5}>0$. This implies the existence of a constant $C_{7}>0$ such that for $h_{0}>0$ small enough and for all $h \in\left(0, h_{0}\right]$,

$$
\left\|\tilde{u}_{h}^{(1)}\right\|_{L^{2}(\dot{\Omega})}+\left\|d \tilde{u}_{h}^{(1)}\right\|_{L^{2}(\dot{\Omega})}+\left\|d^{*} \tilde{u}_{h}^{(1)}\right\|_{L^{2}(\dot{\Omega})} \leq \frac{C_{7}}{h} .
$$

Since $\varphi-f_{+} \geq-C_{8} h \ln \frac{1}{h}$ for some constant $C_{8}$, there exists $N_{0}>0$ such that:

$$
\left\|e^{\frac{f_{+}}{h}} u_{h}^{(1)}\right\|_{L^{2}(\dot{\Omega})}+\left\|d\left(e^{\frac{f_{+}}{h}} u_{h}^{(1)}\right)\right\|_{L^{2}(\dot{\Omega})}+\left\|d^{*}\left(e^{\frac{f_{+}}{h}} u_{h}^{(1)}\right)\right\|_{L^{2}(\dot{\Omega})}=O\left(h^{-N_{0}}\right) .
$$

One has in particular, owing to the trace result (126) stated in Proposition 75 and since $f_{+}=f-f(z)$ on $\Gamma_{1}$,

$$
\left\|e^{\frac{f-f(z)}{h}} u_{h}^{(1)}\right\|_{L^{2}\left(\Gamma_{1}\right)}=O\left(h^{-N_{0}}\right)
$$

Step 2: Agmon estimate in $\dot{\Omega}$.

One follows the same approach as in step 1 but with the function

$$
\varphi=\left\{\begin{array}{l}
\Psi-C h \ln \frac{\Psi}{h}, \text { if } \Psi>C h, \\
\Psi-C h \ln C, \text { if } \Psi \leq C h,
\end{array}\right.
$$


where the constant $C>1$ will be fixed later on, and with the level sets:

$$
\Omega_{-}=\{x \in \dot{\Omega} \text { s.t. } \Psi(x) \leq C h\} \quad \text { and } \quad \Omega_{+}=\dot{\Omega} \backslash \Omega_{-} .
$$

Applying formula (158) then leads to (note that $\left|\tilde{u}_{h}^{(1)}\right| \leq e^{C}\left|u_{h}\right|$ on $\Omega_{-}, \partial_{n} f<0$ on $\Gamma_{0}$ and $\partial_{n} f>0$ on $\Gamma_{2}$ ):

$$
\begin{aligned}
C_{2}(C) h\left(1+\int_{\Gamma_{1}}\left\langle\tilde{u}_{h}^{(1)}, \tilde{u}_{h}^{(1)}\right\rangle_{T_{\sigma}^{*} \Omega} d \sigma\right) \geq & \left\|h d \tilde{u}_{h}^{(1)}\right\|_{L^{2}(\dot{\Omega})}^{2}+\left\|h d^{*} \tilde{u}_{h}^{(1)}\right\|_{L^{2}(\dot{\Omega})}^{2} \\
& +\left\langle\left(|\nabla f|^{2}-|\nabla \varphi|^{2}\right) \tilde{u}_{h}^{(1)}, \tilde{u}_{h}^{(1)}\right\rangle_{L^{2}(\dot{\Omega})}-C_{1} h\left\|\tilde{u}_{h}^{(1)}\right\|_{L^{2}\left(\Omega_{+}\right)}^{2}
\end{aligned}
$$

where $\tilde{u}_{h}^{(1)}:=e^{\frac{\varphi}{h}} u_{h}^{(1)}$, the constant $C_{1}$ is independent of $C$, whereas $C_{2}$ is a constant depending on $C$. Besides, due to the relations

$$
\Psi=f-f(z) \text { on } \Gamma_{1} \text { and } e^{\frac{\varphi}{h}} \leq e^{\frac{\Psi}{h}} \text { on } \dot{\Omega},
$$

the trace estimate obtained in 169 implies

$$
\int_{\Gamma_{1}}\left\langle\tilde{u}_{h}^{(1)}, \tilde{u}_{h}^{(1)}\right\rangle_{T_{\sigma}^{*} \Omega} d \sigma=O\left(h^{-2 N_{0}}\right)
$$

Injecting 171) in 170 then gives

$$
\begin{aligned}
& \left\|h d \tilde{u}_{h}^{(1)}\right\|_{L^{2}(\dot{\Omega})}^{2}+\left\|h d^{*} \tilde{u}_{h}^{(1)}\right\|_{L^{2}(\dot{\Omega})}^{2} \\
& +\left\langle\left(|\nabla f|^{2}-|\nabla \varphi|^{2}\right) \tilde{u}_{h}^{(1)}, \tilde{u}_{h}^{(1)}\right\rangle_{L^{2}(\dot{\Omega})}-C_{1} h\left\|\tilde{u}_{h}^{(1)}\right\|_{L^{2}\left(\Omega_{+}\right)}^{2}=O\left(h^{1-2 N_{0}}\right) .
\end{aligned}
$$

Since moreover $|\nabla \Psi|^{2} \leq|\nabla f|^{2}$ (see 77 ) and $f$ has no critival point in $\bar{\Omega}$, one gets:

$$
|\nabla f|^{2}-|\nabla \varphi|^{2} \geq|\nabla f|^{2}-|\nabla \Psi|^{2}\left(1-\frac{C h}{\Psi}\right) \geq C h \frac{|\nabla f|^{2}}{\Psi} \geq C C_{3} h \text { on } \Omega_{+}
$$

where $C_{3}>0$ is independent of $C$. Since $|\nabla f|^{2} \geq|\nabla \Psi|^{2}=|\nabla \varphi|^{2}$ a.e. on $\Omega_{-}$, adding the term $\left(C C_{3}-C_{1}\right) h\left\|\tilde{u}_{h}^{(1)}\right\|_{L^{2}\left(\Omega_{-}\right)}^{2}$ to 172 leads to

$$
\left\|h d \tilde{u}_{h}^{(1)}\right\|_{L^{2}(\dot{\Omega})}^{2}+\left\|h d^{*} \tilde{u}_{h}^{(1)}\right\|_{L^{2}(\dot{\Omega})}^{2}+\left(C C_{3}-C_{1}\right) h\left\|\tilde{u}_{h}^{(1)}\right\|_{L^{2}(\dot{\Omega})}^{2}=O\left(h^{1-2 N_{0}}\right)
$$

Now, since $\varphi-\Psi \geq-C_{4} h \ln \frac{1}{h}$, taking $C>\frac{C_{1}}{C_{3}}$, there exists $N_{1}>0$ such that:

$$
\left\|e^{\frac{\Psi}{h}} u_{h}^{(1)}\right\|_{L^{2}(\dot{\Omega})}+\left\|d\left(e^{\frac{\Psi}{h}} u_{h}^{(1)}\right)\right\|_{L^{2}(\dot{\Omega})}+\left\|d^{*}\left(e^{\frac{\Psi}{h}} u_{h}^{(1)}\right)\right\|_{L^{2}(\dot{\Omega})}=O\left(h^{-N_{1}}\right) .
$$

This concludes the proof of 157 .

\subsection{Comparison of the eigenform $u_{h, i}^{(1)}$ and its WKB approximation}

Throughout this section, one assumes [H1], [H2] and [H3]. In all this section, we consider, for a fixed critical point $z_{i}$, an ensemble of sets $\mathcal{S}_{M, i}$ associated with $z_{i}$ (see Definition 82 and an $L^{2}$-normalized eigenform $u_{h, i}^{(1)}$ of $\Delta_{f, h}^{M,(1)}\left(\dot{\Omega}_{i}\right)$ associated with the eigenvalue 0 , as introduced at the end of Section 4.1.3. For the ease of notation, we drop the subscript $i$ in all this section. 


\subsubsection{Construction of the WKB expansion of $u_{h}^{(1)}$}

Let $z$ be a local minimum of $\left.f\right|_{\partial \Omega}$. Before going through a rigorous construction of a WKB expansion $u_{z, w k b}^{(1)}$ of $u_{h}^{(1)}$ in a neighborhood of $z$, let us explain formally how we proceed. Let us recall that the 1 -form $u_{h}^{(1)}$ satisfies:

$$
\left\{\begin{array}{l}
\Delta_{f, h}^{(1)} u_{h}^{(1)}=0 \text { in } \dot{\Omega}, \\
\mathbf{t} u_{h}^{(1)}=0 \text { and } \mathbf{t} d_{f, h}^{*} u_{h}^{(1)}=0 \text { on } \Gamma_{1},
\end{array}\right.
$$

plus additional boundary conditions on $\Gamma_{0} \cup \Gamma_{2}$ that we do not recall since the objective is to approximate $u_{h}^{(1)}$ in a neighborhood of $\Sigma$ in $\overline{\dot{\Omega}}$ (where we recall $\Sigma$ is an open subset of $\partial \Omega$ containing $z$ and such that $\bar{\Sigma} \subset \Gamma_{1}$, see 155$)$. The behavior of $u_{h}^{(1)}$ in a neighborhood of $\Gamma_{1}$ exhibited in Proposition 87 suggests to take $u_{z, w k b}^{(1)}$ of the form $u_{z, w k b}^{(1)}(x, h)=a^{(1)}(x, h) e^{-\frac{d_{a}(x, z)}{h}}$ where $a^{(1)}$ is expanded in powers of $h: a^{(1)}(x, h)=$ $\sum_{k \geq 0} a_{k}^{(1)}(x) h^{k}$ and to look for 1-forms $\left(a_{k}^{(1)}\right)_{k \geq 0}$ so that $u_{z, w k b}^{(1)}$ is a nontrivial 1-form satisfying (compare with (174)):

$$
\left\{\begin{array}{l}
\Delta_{f, h}^{(1)} u_{z, w k b}^{(1)}=O\left(h^{\infty}\right) e^{-\frac{d_{a}(\cdot, z)}{h}} \text { in } \dot{\Omega}, \\
\mathbf{t} u_{z, w k b}^{(1)}=0 \text { and } \mathbf{t} d_{f, h}^{*} u_{z, w k b}^{(1)}=O\left(h^{\infty}\right) e^{-\frac{d_{a}(\cdot, z)}{h}} \text { on } \Gamma_{1},
\end{array}\right.
$$

where the meaning of $O\left(h^{\infty}\right)$ is formally $h^{s} O\left(h^{\infty}\right)=o_{h}(1)$ for any $s \in \mathbb{R}$. The boundary conditions in 175 ensures that when cutting suitably a solution to 175 near $\Gamma_{1}$, the resulting 1-form belongs to the form domain of $\Delta_{f, h}^{M,(1)}(\dot{\Omega})$ (this is needed if one wants to approximate $u_{h}^{(1)}$ on $\left.\partial \dot{\Omega}\right)$. Instead of directly trying to solve $(175)$, the construction of $u_{z, w k b}^{(1)}$ can be simply done as follows (see 39, Section 4.2]). Using the complex property, one considers $u_{z, w k b}^{(1)}=d_{f, h} u_{z, w k b}^{(0)}$ where the function $u_{z, w k b}^{(0)}=a^{(0)}(\cdot, h) e^{-\frac{d_{a}(\cdot, z)}{h}}$ where $a^{(0)}(x, h)=\sum_{k \geq 0} a_{k}^{(0)}(x) h^{k}$ for a non trivial family of functions $\left(a_{k}\right)_{k \geq 0}$ such that:

$$
\left\{\begin{array}{l}
\Delta_{f, h}^{(0)} u_{z, w k b}^{(0)}=O\left(h^{\infty}\right) e^{-\frac{d_{a}(\cdot, z)}{h}} \text { in } \dot{\Omega} \\
u_{z, w k b}^{(0)}=e^{-\frac{1}{h} f} \text { on } \Gamma_{1}
\end{array}\right.
$$

This implies the boundary condition: $a^{(0)}=1$ on $\Gamma_{1}$. Then, if $u_{z, w k b}^{(0)}=a^{(0)} e^{-\frac{d_{a}(., z)}{h}}$ is a solution to (176), we set:

$$
u_{z, w k b}^{(1)}=d_{f, h} u_{z, w k b}^{(0)} .
$$

One can easily check that the 1 -form $u_{z, w k b}^{(1)}$ then satisfies 175 and the extra boundary condition $\mathbf{t} d_{f, h}^{*} u_{z, w k b}^{(1)}=O\left(h^{\infty}\right) e^{-\frac{f-f(z)}{h}}$ on $\Gamma_{1}$. Indeed, it holds:

$$
d_{f, h} u_{z, w k b}^{(0)}=e^{-\frac{d_{a}(\cdot, z)}{h}}\left(d\left(f-d_{a}(\cdot, z)\right) a^{(0)}+h d a^{(0)}\right),
$$

which implies $\mathbf{t} u_{z, w k b}^{(1)}=0$ since $a^{(0)}=1$ and

$$
f-d_{a}(\cdot, z)=f(z) \text { on } \Gamma_{1} .
$$

In addition, one has

$$
d_{f, h}^{*} d_{f, h} u_{z, w k b}^{(0)}=\Delta_{f, h}^{(0)} u_{z, w k b}^{(0)}=O\left(h^{\infty}\right) e^{-\frac{d_{a}(\cdot, z)}{h}}
$$


which implies $\mathbf{t} d_{f, h}^{*} u_{z, w k b}^{(1)}=O\left(h^{\infty}\right) e^{-\frac{d_{a}(\cdot, z)}{h}}$ and

$$
\Delta_{f, h}^{(1)} d_{f, h} u_{z, w k b}^{(0)}=d_{f, h} \Delta_{f, h}^{(0)} u_{z, w k b}^{(0)}=O\left(h^{\infty}\right) e^{-\frac{d_{a}(\cdot, z)}{h}} .
$$

Thus, the 1-form $u_{z, w k b}^{(1)}$ satisfies 175 .

Expanding in powers of $h$ the function $e^{\frac{d_{a}(x, z)}{h}} \Delta_{f, h}^{(0)}\left(\left(\sum_{k \geq 0} a_{k}^{(0)}(x) h^{k}\right) e^{-\frac{d_{a}(x, z)}{h}}\right), u_{z, w k b}^{(0)}$ is a solution to 176 if it holds:

$$
\left|\nabla d_{a}(x, z)\right|=|\nabla f(x)|, \text { for } x \in \dot{\Omega},
$$

which is satisfied at least in a neighborhood of $z$ (see Proposition 71 and if $\left(a_{k}^{(0)}\right)_{k \geq 0}$ satisfies the following transport equations, defined recursively by:

$$
\begin{cases}(\Delta \Phi-\Delta f+2 \nabla \Phi \cdot \nabla) a_{0}^{(0)}=0 & \text { in } \dot{\Omega} \\ (\Delta \Phi-\Delta f+2 \nabla \Phi \cdot \nabla) a_{k+1}^{(0)}=\Delta a_{k}^{(0)} & \text { in } \dot{\Omega}, \forall k \geq 0\end{cases}
$$

with boundary conditions $\left\{\begin{array}{ll}a_{0}^{(0)}=1 & \text { on } \Gamma_{1} \\ a_{k}^{(0)}=0 & \text { on } \Gamma_{1}, \forall k \geq 1\end{array}\right.$. The equation (178) together with the boundary condition (177) justify a posteriori the choice of the function $d_{a}(\cdot, z)$ in the exponential for the ansatz on $u_{z, w k b}^{(1)}$. Let us mention that $\partial_{n} f>0$ on $\Gamma_{1}$ implies that there exists a non trivial solution $u_{z, w k b}^{(0)}$ to 176 in a neighborhood of $\Gamma_{1}$ since in that case the transport equations (179) are non degenerate. Let us now justify rigorously the construction of the WKB expansion $u_{z, w k b}^{(1)}$ of $u_{h}^{(1)}$, which is, in view of 178 and $(179)$, possible near $\Gamma_{1}$.

A preliminary construction.

Let $\Phi$ be the solution to the eikonal equation (107) on a neighborhood $V_{\partial \Omega}$ of the boundary $\partial \Omega$. Let us introduce the formal transport operator

$$
T:=\Delta \Phi-\Delta f+2 \nabla \Phi \cdot \nabla .
$$

Let us consider the solutions to the following transport equations, defined recursively by

$$
\begin{cases}T a_{0}=0 & \text { in } V_{\partial \Omega} \\ T a_{k+1}=\Delta a_{k} & \text { in } V_{\partial \Omega}, \quad \forall k \geq 0,\end{cases}
$$

with boundary conditions

$$
\begin{cases}a_{0}=1 & \text { on } \partial \Omega \\ a_{k}=0 & \text { on } \partial \Omega, \quad \forall k \geq 1 .\end{cases}
$$

For a fixed $k$, the transport equation can be solved locally around each $z \in \partial \Omega$ thanks to the condition $\partial_{n} \Phi=-\partial_{n} f<0$ on $\partial \Omega$ and thus on a neighborhood of $\partial \Omega$ (independent of $k$ ) using a compactness argument. Therefore, up to choosing a smaller neighborhood $V_{\partial \Omega}$ of $\partial \Omega$ in $\bar{\Omega}$, there exists a unique sequence of $C^{\infty}\left(V_{\partial \Omega}\right)$ functions $\left(a_{k}\right)_{k \geq 0}$ solution to $180-181$.

There exists a function $a=a(x, h)$ (called a resummation of the formal symbol $\left.\sum_{k=0}^{+\infty} a_{k} h^{k}\right) C^{\infty}$ and uniformly bounded together with all its derivatives such that

$$
a(x, h)=1 \text { on } \partial \Omega \quad \text { and } \quad a(x, h) \sim \sum_{k=0}^{+\infty} a_{k}(x) h^{k} .
$$


This means that $a-\sum_{k=0}^{+\infty} a_{k} h^{k}$ is $O\left(h^{\infty}\right)$ in the following sense: for all compact $K$ in $V_{\partial \Omega}$, for all $\alpha \in \mathbb{N}^{d}$, for all $N \in \mathbb{N}$,

$$
\left\|\partial_{x}^{\alpha}\left(a-\sum_{k=0}^{N} a_{k}(x) h^{k}\right)\right\|_{L^{\infty}(K)} \leq C_{K, \alpha, N} h^{N+1} .
$$

Such a construction is standard and can be found in [25] or in [39], where it is done using a Borel summation. Moreover $a$ is unique up to a term of order $O\left(h^{\infty}\right)$. Let us now define on $V_{\partial \Omega}$ :

$$
u_{w k b}^{(0)}(x, h):=a(x, h) e^{-\frac{\Phi}{h}}
$$

By construction of the sequence $\left(a_{k}\right)_{k \geq 0}$, the function $u_{w k b}^{(0)}$ solves

$$
\left\{\begin{aligned}
\Delta_{f, h}^{(0)} u_{w k b}^{(0)} & =O\left(h^{\infty}\right) e^{-\frac{\Phi}{h}} \text { in } V_{\partial \Omega}, \\
u_{w k b}^{(0)} & =e^{-\frac{\Phi}{h}}=e^{-\frac{f}{h}} \text { on } \partial \Omega,
\end{aligned}\right.
$$

where $O\left(h^{\infty}\right)$ is defined in $(182)$. Indeed, using (44), $|\nabla f|^{2}=|\nabla \Phi|^{2}$ on $V_{\partial \Omega}$, and the equations 180 satisfied by $\left(a_{k}\right)_{k \geq 0}$,

$$
\begin{aligned}
e^{\frac{\Phi}{h}} \Delta_{f, h}^{(0)} u_{w k b}^{(0)}= & -h^{2} \Delta a(x, h)+h[a(x, h) \Delta \Phi+2 \nabla \Phi \cdot \nabla a(x, h)]-a(x, h)|\nabla \Phi|^{2} \\
& +a(x, h)|\nabla f|^{2}-h a(x, h) \Delta f \\
\sim & h T a_{0}+h^{2} \sum_{k=0}^{+\infty} h^{k}\left(T a_{k+1}-\Delta a_{k}\right) \\
= & O\left(h^{\infty}\right) .
\end{aligned}
$$

In addition, it holds $u_{w k b}^{(0)}=e^{-\frac{\Phi}{h}}$ on $\partial \Omega$ since $a(x, h)=1$ on $\partial \Omega$. Let us now define on $V_{\partial \Omega}$ :

The 1-form $u_{w k b}^{(1)}$ satisfies:

$$
u_{w k b}^{(1)}:=d_{f, h} u_{w k b}^{(0)}
$$

$$
\left\{\begin{array}{r}
\Delta_{f, h}^{(1)} u_{w k b}^{(1)}=O\left(h^{\infty}\right) e^{-\frac{\Phi}{h}} \text { in } V_{\partial \Omega} \\
\mathbf{t} u_{w k b}^{(1)}=0 \text { on } \partial \Omega \\
\mathbf{t} d_{f, h}^{*} u_{w k b}^{(1)}=O\left(h^{\infty}\right) e^{-\frac{\Phi}{h}} \text { on } \partial \Omega
\end{array}\right.
$$

where $O\left(h^{\infty}\right)$ is defined in 182 . Indeed, one has $\mathbf{t} u_{w k b}^{(1)}=\mathbf{t} d_{f, h} u_{w k b}^{(0)}=d_{f, h} \mathbf{t} u_{w k b}^{(0)}=$ $d_{f, h} \mathbf{t}\left(a(x, h) e^{-\frac{\Phi}{h}}\right)=d_{f, h} \mathbf{t} e^{-\frac{f}{h}}=d_{f, h} e^{-\frac{f}{h}}=0$ since $a(x, h)=1$ and $\Phi=f$ on $\partial \Omega$. Moreover $\mathbf{t} d_{f, h}^{*} u_{w k b}^{(1)}=\mathbf{t} d_{f, h}^{*} d_{f, h} u_{w k b}^{(0)}=\mathbf{t} \Delta_{f, h}^{(0)} u_{w k b}^{(0)}=O\left(h^{\infty}\right) e^{-\frac{\Phi}{h}}$. Finally, $\Delta_{f, h}^{(1)} u_{w k b}^{(1)}=$ $\Delta_{f, h}^{(1)} d_{f, h} u_{w k b}^{(0)}=d_{f, h} \Delta_{f, h}^{(0)} u_{w k b}^{(0)}=(h d+d f \wedge) O\left(h^{\infty}\right) e^{-\frac{\Phi}{h}}=O\left(h^{\infty}\right) e^{-\frac{\Phi}{h}}$.

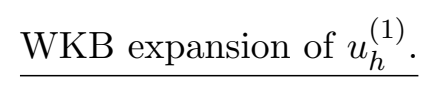

Let $z$ be a local minimum of $\left.f\right|_{\partial \Omega}$. Let us now define the WKB expansion of $u_{h}^{(1)}$ on $V_{\partial \Omega}$ by:

$$
u_{z, w k b}^{(1)}:=e^{\frac{f(z)}{h}} u_{w k b}^{(1)}=e^{\frac{f(z)}{h}} d_{f, h} u_{w k b}^{(0)}=d_{f, h}\left(a(\cdot, h) e^{-\frac{\Phi-f(z)}{h}}\right) .
$$

One recalls (see Proposition 71) that for any smooth open domain $\Gamma$ such that $\bar{\Gamma} \subset \Gamma_{1}$ and $z \in \Gamma$, there exists a neighborhood of $\Gamma$ in $\bar{\Omega}$, denoted by $V_{\Gamma} \subset V_{\partial \Omega} \cap\left(\Gamma_{1} \cup \dot{\Omega}\right)$, such that for all $x \in V_{\Gamma}$,

$$
\Psi(x)=d_{a}(x, z)=\Phi(x)-f(z)
$$


Lemma 89. Let us assume that the hypotheses [H1] and [H3] hold. Let us consider z a local minimum of $\left.f\right|_{\partial \Omega}$ as introduced in hypothesis [H2]. The 1-form $u_{z, w k b}^{(1)}$ satisfies

$$
\left\{\begin{aligned}
& \Delta_{f, h}^{(1)} u_{z, w k b}^{(1)}= O\left(h^{\infty}\right) e^{-\frac{\Phi-f(z)}{h}} \text { in } V_{\partial \Omega}, \\
& \mathbf{t} u_{z, w k b}^{(1)}=0 \text { on } \partial \Omega \\
& \mathbf{t} d_{f, h}^{*} u_{z, w k b}^{(1)}=O\left(h^{\infty}\right) e^{-\frac{\Phi-f(z)}{h}} \text { on } \partial \Omega
\end{aligned}\right.
$$

where $O\left(h^{\infty}\right)$ is defined in 182 . For any $\chi \in C_{c}^{\infty}\left(V_{\Gamma}\right)$ such that $\chi=1$ on a neighborhood of $z$, it holds: in the limit $h \rightarrow 0$,

$$
\int_{\Omega}\left|\chi(x) u_{z, w k b}^{(1)}(x)\right|^{2} d x=C_{z, w k b}^{2} h^{\frac{d+1}{2}}(1+O(h)),
$$

where

$$
C_{z, w k b}:=\pi^{\frac{d-1}{4}} \frac{\sqrt{2 \partial_{n} f(z)}}{\left(\left.\operatorname{det} \operatorname{Hess} f\right|_{\partial \Omega}(z)\right)^{\frac{1}{4}}} .
$$

Furthermore, there exists $C>0$ such that for $h$ small enough,

$$
\left\|\chi u_{z, w k b}^{(1)}\right\|_{H^{1}(\Omega)} \leq C h^{-1}
$$

and $\mathcal{Q}_{f, h}^{M,(1)}(\dot{\Omega})\left(\chi u_{z, w k b}^{(1)}\right)=O\left(h^{\infty}\right)$.

Proof. Equation (185) is easily obtained from (183). Let us now prove (186) and (187). Notice that one can write (using 42,)

$$
\begin{aligned}
u_{z, w k b}^{(1)}= & d_{f, h}\left[e^{-\frac{\Phi-f(z)}{h}} \sum_{k=0}^{+\infty} a_{k} h^{k}\right] \\
= & e^{-\frac{f}{h}}(h d) e^{\frac{f}{h}}\left[e^{-\frac{\Phi-f(z)}{h}} \sum_{k=0}^{+\infty} a_{k} h^{k}\right] \\
= & e^{-\frac{\Phi-f(z)}{h}} e^{-\frac{f-\Phi}{h}}(h d) e^{\frac{f-\Phi}{h}}\left[\sum_{k=0}^{+\infty} a_{k} h^{k}\right] \\
= & e^{-\frac{\Phi-f(z)}{h}}\left(d(f-\Phi) \wedge a_{0}\right) \\
& +e^{-\frac{\Phi-f(z)}{h}}\left[h d \sum_{k=0}^{+\infty} a_{k} h^{k}+d(f-\Phi) \wedge \sum_{k=1}^{+\infty} a_{k} h^{k}\right] .
\end{aligned}
$$

Recall that the function $\chi$ is supported in $V_{\Gamma}$ and the function $x \mapsto \Phi(x)-f(z)$ has a unique minimum on $V_{\Gamma}$ which is $z$ since $\Phi(x)-f(z)=d_{a}(x, z) \geq 0$ on $V_{\Gamma}$. Therefore, in the limit $h \rightarrow 0$ :

$$
\sqrt{\int_{\Omega}\left|\chi(x) u_{z, w k b}^{(1)}(x)\right|^{2} d x}=\sqrt{\int_{\Omega}\left|e^{-\frac{\Phi-f(z)}{h}} \chi d(f-\Phi) \wedge a_{0}\right|^{2}}(1+O(h)) .
$$

Additionaly, since $\chi(z)=1$ and $|d(f-\Phi)(z)|^{2}=|\nabla(f-\Phi)(z)|^{2}=\left|\nabla_{T}(f-\Phi)(z)\right|^{2}+$ $\left(2 \partial_{n} f(z)\right)^{2}=\left(2 \partial_{n} f(z)\right)^{2}$, one gets using Laplace's method

$$
\int_{\Omega}\left|e^{-\frac{\Phi-f(z)}{h}} \chi d(f-\Phi) \wedge a_{0}\right|^{2}=(\pi h)^{\frac{d+1}{2}} \frac{2 \partial_{n} f(z)}{\pi \sqrt{\left.\operatorname{det} \operatorname{Hess} f\right|_{\partial \Omega}(z)}}(1+O(h)) .
$$


Let us give more details on how to obtain 190$)$. Recall that on $\operatorname{supp}(\chi), \Phi-f(z)=$ $f_{+}+f_{-}, f-\Phi=f-\Psi-f(z)=-2 f_{-}$and, on $\operatorname{supp}(\chi) \cap \partial \Omega, \partial_{n} f=-\partial_{n} f_{-}=\left|\nabla f_{-}\right|$. Thus, using the coordinate set introduced in Definition 77 and the co-area formula $d x=\frac{d \sigma_{\Sigma_{\eta}}}{\left|\nabla f_{-}\right|} d \eta($ see for example $|1|)$

$$
\begin{aligned}
& \int_{\Omega}\left|e^{-\frac{\Phi(x)-f(z)}{h}} \chi(x) d(f-\Phi)(x) \wedge a_{0}(x)\right|^{2} d x=4 \int_{-\alpha}^{0} e^{-2 \frac{\eta}{h}} \int_{\Sigma_{\eta}} e^{-2 \frac{f_{+}}{h}} \chi^{2} a_{0}^{2}\left|\nabla f_{-}\right| d \sigma_{\Sigma_{\eta}} d \eta \\
& =4 \int_{-\alpha}^{0} e^{-2 \frac{\eta}{h}} \int_{\partial \Omega} e^{-2 \frac{f_{+}\left(x^{\prime}, 0\right)}{h}} \chi^{2}\left(x^{\prime}, \eta\right) a_{0}^{2}\left(x^{\prime}, \eta\right)\left|\nabla f_{-}\right|\left(x^{\prime}, \eta\right) j\left(x^{\prime}, \eta\right) d \sigma_{\partial \Omega}\left(x^{\prime}\right) d \eta
\end{aligned}
$$

where $\Sigma_{\eta}=\left\{x, f_{-}(x)=-\eta\right\}, \sigma_{\Sigma_{\eta}}$ is the Lebesgue measure on $\Sigma_{\eta}$. In the last equality, $j\left(x^{\prime}, \eta\right)$ is the Jacobian of the parametrization of $\Sigma_{\eta}$ by $x^{\prime} \in \partial \Omega$. Using the Laplace formula, for any $\eta \in\left[-\eta_{0}, 0\right]$ with $\eta_{0}>0$ sufficiently small so that $\chi^{2}(z, \eta) \neq 0$ for all $\eta \in\left[-\eta_{0}, 0\right]$, one has

$$
\begin{aligned}
& \int_{\partial \Omega} e^{-2 \frac{f_{+}\left(x^{\prime}, 0\right)}{h}} \chi^{2}\left(x^{\prime}, \eta\right) a_{0}^{2}\left(x^{\prime}, \eta\right)\left|\nabla f_{-}\right|\left(x^{\prime}, \eta\right) j\left(x^{\prime}, \eta\right) d \sigma_{\partial \Omega}\left(x^{\prime}\right) \\
& =(\pi h)^{\frac{d-1}{2}}\left(\operatorname{det} \operatorname{Hess} f_{+}(z)\right)^{-1 / 2} \chi^{2}(z, \eta)\left|\nabla f_{-}\right|(z, \eta) j(z, \eta) a_{0}^{2}(z, \eta)(1+O(h))
\end{aligned}
$$

where $O(h)$ is a function of $\eta$ and $h$ with $L^{\infty}$ norm in $\eta \in\left[0, \eta_{0}\right]$ bounded from above by a constant times $h$ (thanks to the regularity of the involved terms), for sufficiently small $h$. Thus, using again Laplace's method:

$$
\begin{aligned}
& \int_{\Omega}\left|e^{-\frac{\Phi-f(z)}{h}} \chi d(f-\Phi) \wedge a_{0}\right|^{2} \\
& =4 \int_{-\alpha}^{0} e^{-2 \frac{\eta}{h}}(\pi h)^{\frac{d-1}{2}}\left(\operatorname{detHess} f_{+}(z)\right)^{-1 / 2} \chi^{2}(z, \eta) a_{0}^{2}(z, \eta)\left|\nabla f_{-}\right|(z, \eta) j(z, \eta) d \eta(1+O(h)) \\
& =2 h(\pi h)^{\frac{d-1}{2}}\left(\operatorname{detHess} f_{+}(z)\right)^{-1 / 2} \chi^{2}(z, 0) a_{0}^{2}(z, 0)\left|\nabla f_{-}\right|(z, 0) j(z, 0)(1+O(h))
\end{aligned}
$$

Since $\chi(z, 0)=1, a_{0}(z, 0)=1$, Hess $f_{+}(z)=\left.\operatorname{Hess} f\right|_{\partial \Omega}(z)$ and $j(z, 0)=1$, this concludes the proof of 190, and thus of 186-(187).

Now, writing

$$
u_{z, w k b}^{(1)}=e^{-\frac{\Phi-f(z)}{h}}[d(f-\Phi) \wedge a(\cdot, h)+h d a(\cdot, h)],
$$

and noticing that $\Phi-f(z) \geq 0$ on supp $\chi$, one has $\left\|\chi u_{z, w k b}^{(1)}\right\|_{H^{1}(\Omega)} \leq C h^{-1}$.

It remains to prove the last statement. Using the fact that $\operatorname{supp}\left[\Delta_{f, h}^{(1)}, \chi\right] \subset \operatorname{supp} \chi$, $\Phi-f(z)=\Psi \geq c^{\prime}>0$ on $\operatorname{supp} \nabla \chi$ and 185 , one gets

$$
\begin{aligned}
\Delta_{f, h}^{(1)}\left(\chi u_{z, w k b}^{(1)}\right) & =\chi \Delta_{f, h}^{(1)}\left(u_{z, w k b}^{(1)}\right)+\left[\Delta_{f, h}^{(1)}, \chi\right]\left(u_{z, w k b}^{(1)}\right) \\
& =O\left(h^{\infty}\right)+O\left(e^{-\frac{c}{h}}\right)=O\left(h^{\infty}\right) .
\end{aligned}
$$

Therefore, from the Cauchy-Schwartz inequality, one has $\left\langle\chi u_{z, w k b}^{(1)}, \Delta_{f, h}^{(1)}\left(\chi u_{z, w k b}^{(1)}\right)\right\rangle_{L^{2}(\dot{\Omega})}=$ $O\left(h^{\infty}\right)$. The fact that $\mathcal{Q}_{f, h}^{M,(1)}(\dot{\Omega})\left(\chi u_{z, w k b}^{(1)}\right)=O\left(h^{\infty}\right)$ then follows from an integration by parts and the boundary conditions in 185 . 


\subsubsection{A first estimate of the accuracy of the WKB approximation}

Recall that $z \in\left\{z_{1}, \ldots, z_{n}\right\}$ is a local minimum of $\left.f\right|_{\partial \Omega}$ and that $u_{h}^{(1)}$ is a $L^{2}$-normalized eigenform of $\Delta_{f, h}^{M,(1)}(\dot{\Omega})$ associated with the eigenvalue 0 . The objective of this section is to prove that $u_{h}^{(1)}$ is accurately approximated by the function $u_{z, w k b}^{(1)}$ defined in the previous section. The computations below are inspired by those made in [39, Chapter 4] where the authors were adapting 35,40 to manifolds with boundary. The novelty is that we compare the two 1-forms in a neighborhood of $B_{z}$, instead of a neighborhood of $z$.

Take two smooth open sets $\Gamma_{S t} \subset \Gamma_{S t}^{\prime} \subset \Gamma_{1}$ which are strongly stable (see Definition 79 and Proposition 80 and such that, for some positive $\varepsilon,\left(\Gamma_{S t}+B(0, \varepsilon)\right) \cap \partial \dot{\Omega} \subset \Gamma_{S t}^{\prime}$ and $\left(\Gamma_{S t}^{\prime}+B(0, \varepsilon)\right) \cap \partial \dot{\Omega} \subset \Gamma_{1}$, see Figure 16 . The fact that $\Gamma_{S t}$ and $\Gamma_{S t}^{\prime}$ are strongly stable and thus that $V_{\Gamma_{S t}}$ and $V_{\Gamma_{S t}}^{\prime}$ are stable under the dynamics 193 -see below- will actually be needed only to get refined estimates in Section 4.4.3. Let us now consider the system of coordinate $\left(x^{\prime}, x_{d}\right)$ (see Definition 77 which is well defined on $V_{\Gamma_{1}}$ by assumption (see item 2 in Proposition 81). Let us introduce the Lipschitz sets $V_{\Gamma_{S t}}$ and $V_{\Gamma_{S t}^{\prime}}$

$$
V_{\Gamma_{S t}}=\left\{\left(x^{\prime}, x_{d}\right) \in \Gamma_{S t} \times(-a, 0)\right\} \text { and } V_{\Gamma_{S t}^{\prime}}=\left\{\left(x^{\prime}, x_{d}\right) \in \Gamma_{S t}^{\prime} \times\left(-a^{\prime}, 0\right)\right\}
$$

where $0<a<a^{\prime}$ are small enough so that $V_{\Gamma_{S t}} \subset V_{\Gamma_{S t}^{\prime}} \subset V_{\Gamma_{1}}$. By construction, there exists $\varepsilon>0$ such that $V_{\Gamma_{S t}}+B(0, \varepsilon) \subset V_{\Gamma_{S t}^{\prime}}$ and $V_{\Gamma_{S t}^{\prime}}+B(0, \varepsilon) \subset V_{\Gamma_{1}} \cap\left(\dot{\Omega} \cup \Gamma_{1}\right)$ (see again Figure 16 for a schematic representation of these sets). In addition $V_{\Gamma_{S t}} \cap \Gamma_{1}=\Gamma_{S t}$ and $V_{\Gamma_{S t}^{\prime}} \cap \Gamma_{1}=\Gamma_{S t}^{\prime}$. Moreover, $a$ and $a^{\prime}$ can be chosen sufficiently small so that the sets $\overline{V_{\Gamma_{S t}}}$ and $\overline{V_{\Gamma_{S t}^{\prime}}}$ are stable under the dynamics

$$
x^{\prime}(t)= \begin{cases}-\nabla \Phi(x(t)) & \text { on } \Omega \\ -\nabla_{T} \Phi(x(t)) & \text { on } \partial \Omega .\end{cases}
$$

This stability is a consequence of two facts. First, for $x(t)$ solution to (193), $\frac{d}{d t} f_{-}(x(t))^{\prime}=-\left|\nabla f_{-}(x(t))\right|^{2}$ (since $\nabla \Phi \cdot \nabla f_{-}=\left|\nabla f_{-}\right|^{2}$ on $V_{\Gamma_{1}}$, thanks to 139, and $\nabla_{T} \Phi \cdot \nabla f_{-}=0$ in $\left.\partial \Omega\right)$ so that $\forall t \geq 0, x_{d}(x(0)) \leq x_{d}(x(t)) \leq 0$. Second, by construction, for sufficiently small $a$ and $a^{\prime}$,

$$
\forall x \in \partial V_{\Gamma_{S t}} \text { such that } x^{\prime}(x) \in \partial \Gamma_{S t}, \nabla \Phi(x) \cdot n_{x}\left(V_{\Gamma_{S t}}\right)>0
$$

(where $n\left(V_{\Gamma_{S t}}\right)$ is the unit ouward normal to $\left.V_{\Gamma_{S t}}\right)$. Indeed for any $z \in \partial \Gamma_{S t}$, $\lim _{\sigma \rightarrow z} n_{\sigma}\left(V_{\Gamma_{S t}}\right)=n_{z}\left(\Gamma_{S t}\right)$ (where the limit is taken for $\sigma \in \partial V_{\Gamma_{S t}}$ with $x^{\prime}(\sigma) \in$ $\partial \Gamma_{S t}$ ), see (148) for a proof, and since $\Gamma_{S t}$ is chosen strongly stable, for $z \in \partial \Gamma_{S t}$, $\nabla \Phi(z) \cdot n_{z}\left(V_{\Gamma_{S t}}\right)=\left(\nabla f_{+}+\nabla f_{-}\right) \cdot n_{z}\left(\Gamma_{S t}\right)=\nabla f_{+} \cdot n_{z}\left(\Gamma_{S t}\right)=\left.\nabla f\right|_{\partial \Omega} \cdot n_{z}\left(\Gamma_{S t}\right)>0$. The argument is of course the same for $V_{\Gamma_{S t}^{\prime}}$.

Let us now introduce two smooth cut-off functions $0 \leq \chi \leq \eta \in \mathcal{C}_{c}^{\infty}\left(\dot{\Omega} \cup \Gamma_{1}\right)$ satisfying

$$
\chi=1 \text { in a neighborhood of } \overline{V_{\Gamma_{S t}}}, \quad \operatorname{supp} \chi \subset V_{\Gamma_{S t}^{\prime}}
$$

and $\eta=1$ in a neighborhood of $\overline{V_{\Gamma_{S t}^{\prime}}}, \quad \operatorname{supp} \eta \subset V_{\Gamma_{1}} \cap\left(\dot{\Omega} \cup \Gamma_{1}\right)$.

Notice that by construction, $\eta=0$ on $\Gamma_{2}$. In the following, we moreover assume that $\chi$ and $\eta$ are tensor products in the system of coordinates $\left(x^{\prime}, x_{d}\right)$ (this will actually be needed only to get refined estimates in Section 4.4.3):

$$
\chi\left(x^{\prime}, x_{d}\right)=\chi_{1}\left(x^{\prime}\right) \chi_{d}\left(x_{d}\right) \text { and } \eta\left(x^{\prime}, x_{d}\right)=\eta_{1}\left(x^{\prime}\right) \eta_{d}\left(x_{d}\right) .
$$


Let $\kappa \in\{\chi, \eta\}$. Owing to Lemma 73 , the 1 -form $\kappa u_{h}^{(1)}$ belongs to $\Lambda^{1} H_{T}^{1}(\dot{\Omega})$. The first a priori estimate on $\kappa\left(u_{h}^{(1)}-c(h) u_{z, w k b}^{(1)}\right)$ is the following:

Proposition 90. Let us assume that the hypotheses [H1], [H2] and [H3] hold. For $\kappa \in\{\chi, \eta\}$, one has

$$
\left\|\kappa\left(u_{h}^{(1)}-c_{z}(h) u_{z, w k b}^{(1)}\right)\right\|_{H^{1}(\dot{\Omega})}=O\left(h^{\infty}\right)
$$

where

$$
c_{z}(h)^{-1}=\left\langle u_{h}^{(1)}, \chi u_{z, w k b}^{(1)}\right\rangle_{L^{2}(\dot{\Omega})} .
$$

The 1 -form $u_{h}^{(1)}$ can be chosen such that $c_{z}(h)>0$. Additionally, when $h \rightarrow 0$

$$
c_{z}(h)=C_{z, w k b}^{-1} h^{-\frac{d+1}{4}}\left(1+O\left(h^{\infty}\right)\right),
$$

where $C_{z, w k b}$ is defined by 187 .

Notice that $\left|c_{z}(h)\right|^{-1}$ is equivalent (in the limit $h \rightarrow 0$ ) to $\left\|\kappa u_{z, w k b}^{(1)}\right\|_{L^{2}(\dot{\Omega})}$ (see 186$)$ ), and can thus be simply understood as a normalizing factor.

Proof. Let us first consider the case $\kappa=\chi$, the other case is considered at the end of the proof. One defines

$$
k(h):=\left\langle u_{h}^{(1)}, \chi u_{z, w k b}^{(1)}\right\rangle_{L^{2}(\dot{\Omega})} \in \mathbb{R} .
$$

If $k(h)<0$, then one changes $u_{h}^{(1)}$ to $-u_{h}^{(1)}$ so that one can suppose without loss of generality that

$$
k(h) \geq 0 .
$$

For $h$ small enough, one has (from Proposition 83 , item (iii))

$$
\pi_{\left[0, c h^{3 / 2}\right)}\left(\Delta_{f, h}^{M,(1)}(\dot{\Omega})\right)\left(\chi u_{z, w k b}^{(1)}\right)=k(h) u_{h}^{(1)} .
$$

Let us define

$$
\alpha_{h}:=\chi\left(u_{z, w k b}^{(1)}-k(h) u_{h}^{(1)}\right) .
$$

Thus, the following identity holds for $h$ small enough

$$
\alpha_{h}=k(h)(1-\chi) u_{h}^{(1)}+\pi_{\left[h^{3 / 2},+\infty\right]}\left(\Delta_{f, h}^{M,(1)}(\dot{\Omega})\right)\left(\chi u_{z, w k b}^{(1)}\right) .
$$

Notice that, using Cauchy-Schwarz inequality and Lemma 89, there exist $C>0$ and $h_{0}>0$ such that for all $h \in\left(0, h_{0}\right)$

$$
|k(h)| \leq C h^{\frac{d+1}{4}} .
$$

Therefore, using Lemma 23, Proposition 87 and Lemma 89 we get

$$
\begin{aligned}
\left\|\alpha_{h}\right\|_{L^{2}(\dot{\Omega})}^{2} & \leq 2 k(h)^{2}\left\|(1-\chi) u_{h}^{(1)}\right\|_{L^{2}(\dot{\Omega})}^{2}+2\left\|\pi_{\left[c h^{3 / 2},+\infty\right]}\left(\Delta_{f, h}^{M,(1)}(\dot{\Omega})\right)\left(\chi u_{z, w k b}^{(1)}\right)\right\|_{L^{2}(\dot{\Omega})}^{2} \\
& \leq C h^{\frac{d+1}{2}}\left\|(1-\chi) u_{h}^{(1)} e^{\frac{\Psi}{h}} e^{\frac{-\Psi}{h}}\right\|_{L^{2}(\dot{\Omega})}^{2}+C h^{-3 / 2} \mathcal{Q}_{f, h}^{M,(1)}(\dot{\Omega})\left(\chi u_{z, w k b}^{(1)}\right) \\
& \leq C h^{\frac{d+1}{2}} h^{-N_{0}} e^{-\frac{c}{h}}+C h^{-3 / 2} \mathcal{Q}_{f, h}^{M,(1)}(\dot{\Omega})\left(\chi u_{z, w k b}^{(1)}\right) \\
& =O\left(h^{\infty}\right),
\end{aligned}
$$


with $c:=\inf _{\operatorname{supp}(1-\chi)} \Psi>0$ (since $\chi=1$ near $z$ ) and the integer $N_{0}$ is given by Proposition 87. Moreover, since $d_{f, h}=h d+d f \wedge$ and $d_{f, h}^{*}=h d^{*}+\mathbf{i}_{\nabla f}$, one obtains using the triangular inequality, the Gaffney inequality (121) (since $\alpha_{h} \in H_{T}^{1}(\dot{\Omega})$ ), the fact that $\mathcal{Q}_{f, h}^{M,(1)}(\dot{\Omega})\left(\chi u_{h}^{(1)}\right)=O\left(e^{-\frac{c}{h}}\right)($ from the Agmon estimate $(157))$ and $\mathcal{Q}_{f, h}^{M,(1)}(\dot{\Omega})\left(\chi u_{z, w k b}^{(1)}\right)=$ $O\left(h^{\infty}\right)$,

$$
\begin{aligned}
\left\|\alpha_{h}\right\|_{H^{1}(\dot{\Omega})}^{2} & \leq C\left(\left\|d \alpha_{h}\right\|_{L^{2}(\dot{\Omega})}^{2}+\left\|d^{*} \alpha_{h}\right\|_{L^{2}(\dot{\Omega})}^{2}+\left\|\alpha_{h}\right\|_{L^{2}(\dot{\Omega})}^{2}\right) \\
& \leq C h^{-2}\left(\mathcal{Q}_{f, h}^{M,(1)}(\dot{\Omega})\left(\alpha_{h}\right)+\left\|\alpha_{h}\right\|_{L^{2}(\dot{\Omega})}^{2}\right) \\
& =O\left(h^{\infty}\right) .
\end{aligned}
$$

Moreover since $\left\|\chi u_{h}^{(1)}\right\|_{L^{2}(\dot{\Omega})}=1+O\left(e^{-\frac{c}{h}}\right)$ (from the Agmon estimate 157$)$, by considering $\left\|\chi\left(u_{z, w k b}^{(1)}-k(h) u_{h}^{(1)}\right)\right\|_{L^{2}(\dot{\Omega})}=O\left(h^{\infty}\right)$, one gets:

$$
\begin{aligned}
k(h)^{2} & =\frac{\left\|\chi u_{z, w k b}^{(1)}\right\|_{L^{2}(\dot{\Omega})}^{2}+O\left(h^{\infty}\right)}{2-\left\|\chi u_{h}^{(1)}\right\|_{L^{2}(\dot{\Omega})}^{2}} \\
& =\frac{C_{z, w k b}^{2} h^{\frac{d+1}{2}}+O\left(h^{\infty}\right)}{1+O\left(e^{-\frac{c}{h}}\right)},
\end{aligned}
$$

with $C_{z, w k b}$ given by (187) in Lemma 89 . Therefore, since $k(h) \geq 0, k(h)=C_{z, w k b} h^{\frac{d+1}{4}}(1+$ $\left.O\left(h^{\infty}\right)\right)$. This concludes the proof of (197) for $\kappa=\chi$, by choosing

$$
c_{z}(h):=k(h)^{-1} \text {. }
$$

Let us now deal with the case $\kappa=\eta$. There exists $c>0$ such that, for $h$ sufficiently small,

$$
\begin{aligned}
\left\|\eta\left(u_{z, w k b}^{(1)}-k(h) u_{h}^{(1)}\right)\right\|_{H^{1}(\dot{\Omega})} & \leq\left\|\alpha_{h}\right\|_{H^{1}(\dot{\Omega})}+\left\|(\eta-\chi)\left(u_{z, w k b}^{(1)}-k(h) u_{h}^{(1)}\right)\right\|_{H^{1}(\dot{\Omega})} \\
& \leq O\left(h^{\infty}\right)+\left\|(\eta-\chi) u_{z, w k b}^{(1)}\right\|_{H^{1}(\dot{\Omega})}+\mid k(h)\|\|(\eta-\chi) u_{h}^{(1)} \|_{H^{1}(\dot{\Omega})} \\
& \leq O\left(h^{\infty}\right)+e^{-\frac{c}{h}} .
\end{aligned}
$$

The last inequality is the consequence of two facts. First, $\left\|(\eta-\chi) u_{h}^{(1)}\right\|_{H^{1}(\dot{\Omega})}=e^{-\frac{c}{h}}$ thanks to Proposition 87 and (121) together with the fact that $\chi=\eta$ near $z$. Second, a direct computation shows that

$$
\left\|(\eta-\chi) u_{z, w k b}^{(1)}\right\|_{H^{1}(\dot{\Omega})} \leq C h^{-1} e^{-\frac{\inf _{\operatorname{supp}(\eta-\chi)^{\Psi}}}{h}} \leq e^{-\frac{c}{h}} .
$$

This concludes the proof of Proposition 90 .

The estimate we obtained in Proposition 90 is sufficient to get the result of Theorem 1. The more precise estimates obtained in Section 4.4 .3 are only needed to prove Theorem 2 . 


\subsubsection{A more accurate comparison on the WKB approximation}

The objective of this section is to combine the techniques used to obtain the Agmon estimates of Proposition 87 and the first estimate of the accuracy of the WKB approximation of Proposition 90 in order to obtain a more precise estimate of the latter.

Let us start with estimates which are simple consequences of Propositions 87 and 90 . Notice that, for $\kappa \in\{\chi, \eta\}$, one obviously gets from Proposition 87 the following relation in $\Lambda^{1} H^{1}(\dot{\Omega})$ :

$$
\exists N_{0} \in \mathbb{N}, e^{\frac{\Psi}{h}} \kappa\left(u_{h}^{(1)}-c_{z}(h) u_{z, w k b}^{(1)}\right)=O\left(h^{-N_{0}}\right) .
$$

For the term involving $u_{z, w k b}^{(1)}$, this is due to $\Psi(x)=\Phi(x)-f(z)$ on $\operatorname{supp} \kappa$ and the estimate 199$)$ on $c_{z}(h)$. Let us now set

$$
w_{h}:=\kappa\left(u_{h}^{(1)}-c_{z}(h) u_{z, w k b}^{(1)}\right) .
$$

The 1 -form $w_{h}$ is in $C_{c}^{\infty}\left(\dot{\Omega} \cup \Gamma_{1}\right)$ and satisfies in $\dot{\Omega}$ :

$$
\begin{aligned}
\Delta_{f, h}^{(1)} w_{h} & =\kappa \Delta_{f, h}^{(1)}\left(u_{h}^{(1)}-c_{z}(h) u_{z, w k b}^{(1)}\right)+\left[\Delta_{f, h}^{(1)}, \kappa\right]\left(u_{h}^{(1)}-c_{z}(h) u_{z, w k b}^{(1)}\right) \\
& =-c_{z}(h) \kappa \Delta_{f, h}^{(1)} u_{z, w k b}^{(1)}+\left[\Delta_{f, h}^{(1)}, \kappa\right]\left(u_{h}^{(1)}-c_{z}(h) u_{z, w k b}^{(1)}\right) \\
& =\left(r_{1}+r_{1}^{\prime}\right) e^{-\frac{\Psi}{h}}
\end{aligned}
$$

where, owing to (185) and (199):

$$
r_{1}:=-e^{\frac{\Psi}{h}} c_{z}(h) \kappa \Delta_{f, h}^{(1)} u_{z, w k b}^{(1)}=O\left(h^{\infty}\right)
$$

in $\Lambda^{1} L^{2}(\Omega)$ and, from 200 :

$$
\begin{aligned}
& r_{1}^{\prime}:=e^{\frac{\Psi}{h}}\left[\Delta_{f, h}^{(1)}, \kappa\right]\left(u_{h}^{(1)}-c_{z}(h) u_{z, w k b}^{(1)}\right)=O\left(h^{-N_{0}}\right) \text { in } \Lambda^{1} L^{2}(\Omega) \text { and } \\
& \operatorname{supp} r_{1}^{\prime} \subset \operatorname{supp} \nabla \kappa .
\end{aligned}
$$

Additionally, one gets similarly on the boundary $\Gamma_{1}$ :

$$
\left.\mathbf{t} w_{h}\right|_{\Gamma_{1}}=0 \text { and }\left.\mathbf{t} d_{f, h}^{*} w_{h}\right|_{\Gamma_{1}}=\left(r_{2}+r_{2}^{\prime}\right) e^{-\frac{\Psi}{h}}=\left(r_{2}+r_{2}^{\prime}\right) e^{-\frac{f-f(z)}{h}},
$$

where owing to (185) and 199):

$$
r_{2}:=\left.\mathbf{t} e^{\frac{\Psi}{h}} \kappa d_{f, h}^{*}\left(u_{h}^{(1)}-c_{z}(h) u_{z, w k b}^{(1)}\right)\right|_{\Gamma_{1}}=-\left.\mathbf{t} e^{\frac{\Psi}{h}} \kappa c_{z}(h) d_{f, h}^{*} u_{z, w k b}^{(1)}\right|_{\Gamma_{1}}=O\left(h^{\infty}\right)
$$

in $L^{2}(\partial \Omega)$ and

$$
\begin{aligned}
& r_{2}^{\prime}:=\left.\mathbf{t} e^{\frac{\Psi}{h}} h \mathbf{i}_{\nabla \kappa}\left(u_{h}^{(1)}-c_{z}(h) u_{z, w k b}^{(1)}\right)\right|_{\Gamma_{1}}=O\left(h^{-N_{0}}\right) \text { in } L^{2}(\partial \dot{\Omega}) \text { with } \\
& \operatorname{supp} r_{2}^{\prime} \subset \Gamma_{1} \cap \operatorname{supp} \nabla \kappa .
\end{aligned}
$$

We are now in position to prove the following proposition.

Proposition 91. Let us assume that the hypotheses [H1], [H2] and [H3] hold. One has the following estimate in the limit $h \rightarrow 0$ :

$$
\left\|e^{\frac{\Psi}{h}}\left(u_{h}^{(1)}-c_{z}(h) u_{z, w k b}^{(1)}\right)\right\|_{H^{1}\left(V_{\Gamma_{S t}}\right)}=O\left(h^{\infty}\right),
$$

where $c_{z}(h)$ is defined by (198) and where, we recall, $\Psi(x)=d_{a}(x, z)$ and $V_{\Gamma_{S t}}$ is defined by 192 . 
Proof. As for the proof of Proposition 87, one first proves an estimate along the boundary $\Gamma_{1}$ before propagating it in $V_{\Gamma_{S t}}$.

Step 1. Comparison in $\Gamma_{1}$.

Let us consider $w_{h}$ defined by (201) and the cut-off function $\kappa=\eta$ defined in 195). Like in the first step of the proof of Proposition 87, we are going to prove an estimate of the form 207) with $\Psi$ replaced by $f_{+}$. More precisely, we want to show that

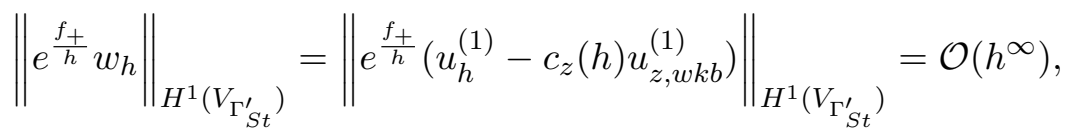

which implies in particular the following estimate along the boundary, since $f_{+}=f-$ $f(z)$ in $\Gamma_{1}$,

$$
\left\|e^{\frac{f-f(z)}{h}}\left(u_{h}^{(1)}-c_{z}(h) u_{z, w k b}^{(1)}\right)\right\|_{H^{1 / 2}\left(\Gamma_{S t}^{\prime}\right)}=\mathcal{O}\left(h^{\infty}\right) .
$$

In the following, we denote (see Figure 16 for a schematic representation of the set $V_{\eta}$ )

$$
V_{\eta}=\operatorname{supp} \eta \text {. }
$$

In the system of coordinates $\left(x^{\prime}, x_{d}\right), x \in V_{\eta}$ if and only if $x^{\prime}(x) \in \operatorname{supp} \eta_{1}$ and $x_{d}(x) \in$ $\operatorname{supp} \eta_{d}$. We recall that $V_{\eta}$ is a compact set of $\dot{\Omega} \cup \Gamma_{1}$. As for the proof of Proposition 87 , we introduce the sets

$$
\Omega_{-}=\left\{x \in V_{\eta} \text { s.t. } f_{+}(x) \leq C h\right\} \text { and } \Omega_{+}=V_{\eta} \backslash \Omega_{-},
$$

and define the Lipschitz function $\varphi: V_{\eta} \rightarrow \mathbb{R}$ by

$$
\varphi=\left\{\begin{array}{c}
f_{+}-C h \ln \frac{f_{+}}{h} \text { if } f_{+}>C h, \\
f_{+}-C h \ln C \text { if } f_{+} \leq C h,
\end{array}\right.
$$

for some constant $C>1$ that will be fixed at the end of this step. Notice for further purposes that

$$
\lim _{h \rightarrow 0}\left\|\varphi-f_{+}\right\|_{L^{\infty}\left(V_{\eta}\right)}=0 .
$$

We recall that in the system of coordinates $\left(x^{\prime}, x_{d}\right), \varphi$ is independent of $x_{d}$.

The reasoning below is based on [40], see also [35, p. 49-52] for a presentation in the case without boundary. According to (208), we want to get an error of the form $\mathcal{O}\left(h^{N}\right)$ with $N$ arbitrary. We are not going to work with the above phase function $\varphi$ as we did in the proof of Proposition 87, but with a phase function $\varphi_{N}$ also depending on some arbitrary $N \in \mathbb{N}$. Let us define

$$
\tilde{w}_{h}=e^{\frac{\varphi_{N}}{h}} w_{h}=e^{\frac{\varphi_{N}}{h}} \eta\left(u_{h}^{(1)}-c_{z}(h) u_{z, w k b}^{(1)}\right) .
$$

Combining the integration by parts formula (158) (with $u=w_{h}$ and $\varphi=\varphi_{N}$ ) with the Green formula (120) (with $u=w_{h}$ and $v=e^{2 \frac{\varphi_{N}}{h}} w_{h}$ ) leads to the estimate

$$
\begin{aligned}
\left\|e^{\frac{\varphi_{N}}{h}} \Delta_{f, h}^{(1)} w_{h}\right\|_{L^{2}\left(V_{\eta}\right)} & \left\|\tilde{w}_{h}\right\|_{L^{2}\left(V_{\eta}\right)}+h\left\|e^{\frac{\varphi_{N}}{h}} \mathbf{t} d_{f, h}^{*} w_{h}\right\|_{L^{2}\left(\Gamma_{1}\right)}\left\|\tilde{w}_{h}\right\|_{L^{2}\left(\Gamma_{1}\right)} \\
\geq & \left\|h d \tilde{w}_{h}\right\|_{L^{2}\left(V_{\eta}\right)}^{2}+\left\|h d^{*} \tilde{w}_{h}\right\|_{L^{2}\left(V_{\eta}\right)}^{2}-h \int_{\Gamma_{1}}\left\langle\tilde{w}_{h}, \tilde{w}_{h}\right\rangle_{T_{\sigma}^{*} \Omega} \partial_{n} f d \sigma \\
& +\left\langle\left(|\nabla f|^{2}-\left|\nabla \varphi_{N}\right|^{2}+h \mathcal{L}_{\nabla f}+h \mathcal{L}_{\nabla f}^{*}\right) \tilde{w}_{h}, \tilde{w}_{h}\right\rangle_{L^{2}\left(V_{\eta}\right)} .
\end{aligned}
$$


Let us explain formally how the function $\varphi_{N}$ is chosen. Roughly speaking, using similar arguments as in the proof of Proposition 87, it is natural to choose $\varphi_{N}=$ $\varphi+N h \ln \frac{1}{h}$ and to try to prove that the left-hand side of $(211)$ is bounded from above by $\mathcal{O}\left(h^{-N_{1}}\right)\left\|\tilde{w}_{h}\right\|_{H^{1}\left(V_{\eta}\right)}$ for some $N_{1} \in \mathbb{N}$ independent of $N$. This would indeed lead to an estimate of the form $\left\|\tilde{w}_{h}\right\|_{H^{1}\left(V_{\eta}\right)}=\mathcal{O}\left(h^{-N_{1}}\right)$ (for some maybe larger $N_{1}$ ) and finally to the desired estimate on $w_{h}$ since $\left\|\tilde{w}_{h}\right\|_{H^{1}\left(V_{\eta}\right)} \simeq h^{-N}\left\|e^{\frac{f_{+}}{h}} w_{h}\right\|_{H^{1}\left(V_{\eta}\right)}$. To get this upper bound, a trace theorem and 202 -206 yield the following estimate from 211):

$\left\|e^{\frac{\varphi_{N}-\Psi}{h}}\right\|_{L^{\infty}\left(V_{\eta}\right)} O\left(h^{\infty}\right)+\left\|e^{\frac{\varphi_{N}-\Psi}{h}}\right\|_{L^{\infty}(\operatorname{supp}(\nabla \eta))} O\left(h^{-N_{1}}\right) \geq\left\|\tilde{w}_{h}\right\|_{H^{1}\left(V_{\eta}\right)} \simeq h^{-N}\left\|e^{\frac{f_{+}}{h}} w_{h}\right\|_{H^{1}\left(V_{\eta}\right)}$

for some $N_{1} \in \mathbb{N}$ independent of $N$. It can be checked that $\left\|e^{\frac{\varphi_{N}-\Psi}{h}}\right\|_{L^{\infty}\left(V_{\eta}\right)}=$ $\left\|e^{\frac{\varphi_{N}-\Psi}{h}}\right\|_{L^{\infty}(\operatorname{supp}(\nabla \eta))}=O\left(h^{-N}\right)$ so that the first term is well controlled, but the second one is of order $O\left(h^{-N-N_{1}}\right)$. These relations suggest a choice of $\varphi_{N}$ satisfying $\varphi_{N} \leq f_{+} \leq \Psi$ on $\operatorname{supp} \nabla \eta$ so that $\left\|e^{\frac{\varphi_{N}-\Psi}{h}}\right\|_{L^{\infty}(\operatorname{supp}(\nabla \eta))}=O(1)$. This would yield the desired estimate $\left\|e^{\frac{f_{+}}{h}} w_{h}\right\|_{H^{1}\left(V_{\eta}\right)}=O\left(h^{N-N_{1}}\right)$.

Let us now enter the rigorous proof. The above considerations (see also 35 , p. 4952]) lead to define, for any $N \in \mathbb{N}$,

$$
\varphi_{N}=\min \left\{\varphi+N h \ln \frac{1}{h}, \psi\right\},
$$

where the Lipschitz function $\psi: V_{\eta} \rightarrow \mathbb{R}$ is defined by the following relation, for some $\varepsilon \in(0,1)$ that will be specified below:

$$
\psi\left(x^{\prime}, x_{d}\right)=\psi\left(x^{\prime}, 0\right)=\min \left\{\varphi\left(y^{\prime}, 0\right)+(1-\varepsilon) d_{a}^{\partial \Omega}\left(x^{\prime}, y^{\prime}\right), y^{\prime} \in \operatorname{supp} \nabla \eta_{1}\right\} .
$$

Here, $d_{a}^{\partial \Omega}\left(x^{\prime}, y^{\prime}\right)$ denotes the Agmon distance associated with $\left.f\right|_{\partial \Omega}$ between $x^{\prime}$ and $y^{\prime}$ along the boundary (see Definition 50), i.e. the distance induced by the metric $\left|\nabla\left(\left.f\right|_{\partial \Omega}\right)\right|^{2} d s^{2}$, where $d s^{2}$ denotes the restriction of the Euclidean metric to the boundary $\partial \Omega$.

Step 1-a: Preliminary estimates on $\varphi_{N}$.

Let us first show that there exists $\varepsilon \in(0,1)$ such that for any $h \in\left(0, h_{0}(N, \varepsilon)\right)$ with $h_{0}=h_{0}(N, \varepsilon)$ small enough,

$$
\varphi_{N}=\varphi+N h \ln \frac{1}{h}<\psi \text { in } V_{\eta} \cap\left\{x^{\prime} \in \overline{\Gamma_{S t}^{\prime}}\right\} .
$$

The proof of (214) is as follows. From (78) applied to $d_{a}^{\partial \Omega}$,

$$
\forall\left(x^{\prime}, y^{\prime}\right) \in \overline{\Gamma_{S t}^{\prime}} \times \operatorname{supp} \nabla \eta_{1}, \quad f_{+}\left(x^{\prime}, 0\right)<f_{+}\left(y^{\prime}, 0\right)+d_{a}^{\partial \Omega}\left(x^{\prime}, y^{\prime}\right) .
$$

The inequality above is strict since if $f_{+}\left(x^{\prime}, 0\right)=f_{+}\left(y^{\prime}, 0\right)+d_{a}^{\partial \Omega}\left(x^{\prime}, y^{\prime}\right)$ for some $\left(x^{\prime}, y^{\prime}\right) \in$ $\overline{\Gamma_{S t}^{\prime}} \times \operatorname{supp} \nabla \eta_{1}$, then there exists a generalized integral curve (in the sense of Definition 65 of $-\nabla\left(\left.f\right|_{\partial \Omega}\right)=-\nabla f_{+}$joining $x^{\prime} \in \overline{\Gamma_{S t}^{\prime}}$ to $y \in \operatorname{supp} \nabla \eta_{1}$ (this is a consequence of Corollary 64 applied to the Agmon distance $d_{a}^{\partial \Omega}$ on $\partial \Omega$ rather than the Agmon distance $d_{a}$ in $\left.\bar{\Omega}\right)$. But since $\Gamma_{S t}^{\prime}$ is strongly stable, any integral curve of $-\nabla f_{+}$remains in $\overline{\Gamma_{S t}^{\prime}}$, and thus cannot reach $y^{\prime}$ which is not in $\overline{\Gamma_{S t}^{\prime}}$ (see 195$)$ ).

From the strict inequality (215), there exists $\varepsilon_{0}>0$ such that for all $\varepsilon \in\left[0, \varepsilon_{0}\right)$,

$$
\forall\left(x^{\prime}, y^{\prime}\right) \in \overline{\Gamma_{S t}^{\prime}} \times \operatorname{supp} \nabla \eta_{1}, \quad f_{+}\left(x^{\prime}, 0\right) \leq f_{+}\left(y^{\prime}, 0\right)+(1-\varepsilon) d_{a}^{\partial \Omega}\left(x^{\prime}, y^{\prime}\right),
$$


and thus, considering the limit $h \rightarrow 0$ (see (210) ) and the infimum over $y^{\prime} \in \operatorname{supp} \nabla \eta_{1}$ of the right-hand side, there exists $\varepsilon>0$ used to define $\psi$ (see (213)) and such that, for sufficiently small $h$,

$$
\forall x^{\prime} \in \overline{\Gamma_{S t}^{\prime}}, \quad f_{+}\left(x^{\prime}, 0\right)<\psi\left(x^{\prime}, 0\right) .
$$

Moreover, since $\lim _{h \rightarrow 0}\left\|\varphi+N h \ln \frac{1}{h}-f_{+}\right\|_{L^{\infty}\left(V_{\eta}\right)}=0$ (thanks to 210), one obtains for $h$ small enough, $\forall x^{\prime} \in \overline{\Gamma_{S t}^{\prime}}, \varphi\left(x^{\prime}, 0\right)+N h \ln \frac{1}{h}<\psi\left(x^{\prime}, 0\right)$, and by definition of $\varphi_{N}$, $\varphi_{N}\left(x^{\prime}, 0\right)=\varphi\left(x^{\prime}, 0\right)+N h \ln \frac{1}{h}$ which leads to 214 for $x=\left(x^{\prime}, 0\right)$, with $x^{\prime} \in \overline{\Gamma_{S t}^{\prime}}$. The fact that $\varphi_{N}$ and $\psi$ do not depend on $x_{d}$ in the system of coordinates $\left(x^{\prime}, x_{d}\right)$ concludes the proof of 214).

Let us now prove that

$$
\exists M<\frac{1}{1-\varepsilon}, \forall x \in V_{\eta},|\nabla \psi(x)| \leq M(1-\varepsilon)\left|\nabla f_{+}(x)\right| .
$$

The triangular inequality applied to $d_{a}^{\partial \Omega}$ leads to the relation (since $\psi\left(x^{\prime}, x_{d}\right)$ does not depend on $x_{d}$ )

$$
\forall x, y \in V_{\eta},\left|\psi\left(x^{\prime}, x_{d}\right)-\psi\left(y^{\prime}, y_{d}\right)\right| \leq(1-\varepsilon) d_{a}^{\partial \Omega}\left(x^{\prime}, y^{\prime}\right) .
$$

where we denote $\left(x^{\prime}, x_{d}\right)$ (resp. $\left.\left(y^{\prime}, y_{d}\right)\right)$ the coordinates of $x$ (resp. $y$ ) in the system of coordinates (136). Let us first show that 217) implies that

$$
\text { for a.e. } x^{\prime} \in V_{\eta} \cap \partial \Omega,\left|\nabla\left(\left.\psi\right|_{\partial \Omega}\right)\left(x^{\prime}\right)\right| \leq(1-\varepsilon)\left|\nabla\left(\left.f\right|_{\partial \Omega}\right)\left(x^{\prime}\right)\right|=(1-\varepsilon)\left|\nabla\left(\left.f_{+}\right|_{\partial \Omega}\right)\left(x^{\prime}\right)\right| \text {. }
$$

Indeed, let us consider a local parametrization in $\mathbb{R}^{d-1}$ of a neighborhood in $\partial \Omega$ of a point $x^{\prime} \in \partial \Omega$. In this local chart, let us consider $y_{\alpha}=x^{\prime}+\alpha \frac{\nabla\left(\left.\psi\right|_{\partial \Omega}\right)}{\left|\nabla\left(\left.\psi\right|_{\partial \Omega}\right)\right|}\left(x^{\prime}\right)$. One has, in the limit $\alpha \rightarrow 0$,

$$
\psi\left(y_{\alpha}^{\prime}, 0\right)-\psi\left(x^{\prime}, 0\right)=\alpha\left|\nabla\left(\left.\psi\right|_{\partial \Omega}\right)\left(x^{\prime}\right)\right|+o(\alpha)
$$

and likewise using the inequality (77) applied to $d_{a}^{\partial \Omega}$ (see also [25, p. 53])

$$
d_{a}^{\partial \Omega}\left(x,{ }^{\prime} y_{\alpha}^{\prime}\right)-d_{a}^{\partial \Omega}\left(x^{\prime}, x^{\prime}\right) \leq \alpha\left|\nabla\left(\left.f\right|_{\partial \Omega}\right)\left(x^{\prime}\right)\right|+o(\alpha) .
$$

By considering the limit $\alpha \rightarrow 0$, one thus deduces (218) from (217).

Now, one can check that, uniformly in $x^{\prime} \in V_{\eta} \cap \partial \Omega$,

$$
\lim _{x_{d} \rightarrow 0}\left|\nabla \psi\left(x^{\prime}, x_{d}\right)\right|=\left|\nabla\left(\left.\psi\right|_{\partial \Omega}\right)\left(x^{\prime}\right)\right| \text { and } \lim _{x_{d} \rightarrow 0}\left|\nabla f_{+}\left(x^{\prime}, x_{d}\right)\right|=\left|\nabla\left(\left.f_{+}\right|_{\partial \Omega}\right)\left(x^{\prime}\right)\right| .
$$

Indeed, using the fact that $\psi$ does not depend on $x_{d}$, one first has almost everywhere (see (137) $)\left|\nabla \psi\left(x^{\prime}, x_{d}\right)\right|=\left|\nabla\left(\left.\psi\right|_{\Sigma_{x_{d}}}\right)\left(x^{\prime}\right)\right|$ where $\forall a>0, \Sigma_{a}=\left\{x \in V_{\eta}, x_{d}(x)=a\right\}$ is endowed with the Riemannian structure induced by the Riemannian structure in $\Omega$. Now, let us consider the smooth diffeomorphism $\Gamma_{x_{d}}: \Sigma_{x_{d}} \rightarrow \partial \Omega$ such that for all $x=\left(x^{\prime}, x_{d}\right) \in \Sigma_{x_{d}}, \Gamma_{x_{d}}(x)=\left(x^{\prime}, 0\right) \in \partial \Omega$. The result 219) on $\psi$ is then a consequence of the fact that $\left.\psi\right|_{\partial \Omega} \circ \Gamma_{x_{d}}=\left.\psi\right|_{\Sigma_{x_{d}}}$ and $\lim _{x_{d} \rightarrow 0}\left\|\Gamma_{x_{d}}-\mathrm{Id}\right\|_{W^{1, \infty}\left(\Sigma_{x_{d}}\right)}=0$ so that the Jacobian associated to the change of metric from $\Sigma_{x_{d}}$ to $\partial \Omega$ converges to Id, uniformly on $\Sigma_{x_{d}}$. The same reasoning show that 219 also holds for $f_{+}$since $f_{+}$does not depend on $x_{d}$.

By combining (218) and (219), one obtains (216) for some $M>1$. Moreover, $M$ can be chosen as close to 1 as needed, up to modifying $\eta$ (and thus $V_{\Gamma_{S t}} \subset V_{\Gamma_{S t}^{\prime}} \subset V_{\eta}$ ) such that for all $x \in V_{\eta},\left\|\Gamma_{x_{d}}-\operatorname{Id}\right\|_{W^{1, \infty}\left(\Sigma_{x_{d}}\right)}$ is as close to 0 as needed. 
Let us finally mention the following inequalities, valid for $h \in\left(0, h_{0}\right)$ with $h_{0}=$ $h_{0}(N, \varepsilon)>0$ small enough:

$$
\begin{aligned}
& \varphi_{N} \leq f_{+}+N h \ln \frac{1}{h} \leq \Psi+N h \ln \frac{1}{h} \quad \text { in } V_{\eta} \\
& \varphi_{N}=\psi \leq \varphi \leq f_{+} \leq \Psi \quad \text { in } V_{\eta} \cap\left\{x^{\prime} \in \operatorname{supp} \nabla \eta_{1}\right\}
\end{aligned}
$$

and since $\Psi=f_{+}+f_{-}>f_{+}$on $\left\{x_{d} \in \operatorname{supp} \eta_{d}^{\prime}\right\}$, one has

$$
\varphi_{N} \leq f_{+}+N h \ln \frac{1}{h} \leq \Psi \quad \text { in } V_{\eta} \cap\left\{x_{d} \in \operatorname{supp} \eta_{d}^{\prime}\right\} .
$$

Step 1-b: Proof of 208.

We are now ready to prove (208). Controlling the left-hand side of 211) using the relations 202 -206) gives

$$
\begin{aligned}
\left\|\left(r_{1}+r_{1}^{\prime}\right) e^{\frac{\varphi_{N}-\Psi}{h}}\right\|_{L^{2}\left(V_{\eta}\right)} & \left\|\tilde{w}_{h}\right\|_{L^{2}\left(V_{\eta}\right)}+\left\|\left(r_{2}+r_{2}^{\prime}\right) e^{\frac{\varphi_{N}-\Psi}{h}}\right\|_{L^{2}\left(\Gamma_{1}\right)}\left\|\tilde{w}_{h}\right\|_{L^{2}\left(\Gamma_{1}\right)} \\
\geq & \left\|h d \tilde{w}_{h}\right\|_{L^{2}\left(V_{\eta}\right)}^{2}+\left\|h d^{*} \tilde{w}_{h}\right\|_{L^{2}\left(V_{\eta}\right)}^{2}-h \int_{\Gamma_{1}}\left\langle\tilde{w}_{h}, \tilde{w}_{h}\right\rangle_{T_{\sigma}^{*} \Omega} \partial_{n} f d \sigma \\
& +\left\langle\left(|\nabla f|^{2}-\left|\nabla \varphi_{N}\right|^{2}+h \mathcal{L}_{\nabla f}+h \mathcal{L}_{\nabla f}^{*}\right) \tilde{w}_{h}, \tilde{w}_{h}\right\rangle_{L^{2}\left(V_{\eta}\right)},
\end{aligned}
$$

where, since $\varphi_{N}-\Psi \leq N h \ln \frac{1}{h}$ (by (220) ) and $r_{i}=\mathcal{O}\left(h^{\infty}\right)$ for $i \in\{1,2\}$ (by (203) and (205)),

$$
\left\|r_{1} e^{\frac{\varphi_{N}-\Psi}{h}}\right\|_{L^{2}\left(V_{\eta}\right)}+\left\|r_{2} e^{\frac{\varphi_{N}-\Psi}{h}}\right\|_{L^{2}\left(\Gamma_{1}\right)}=\mathcal{O}\left(h^{\infty}\right),
$$

and, since $\varphi_{N} \leq \Psi$ on $\operatorname{supp} \nabla \eta$ (by (221)-222) ) and $\operatorname{supp} r_{i}^{\prime} \subset \operatorname{supp} \nabla \eta$ for $i \in\{1,2\}$ (by (204) and (206),

$$
\left\|r_{1}^{\prime} e^{\frac{\varphi_{N}-\Psi}{h}}\right\|_{L^{2}\left(V_{\eta}\right)}+\left\|r_{2}^{\prime} e^{\frac{\varphi_{N}-\Psi}{h}}\right\|_{L^{2}\left(\Gamma_{1}\right)}=\mathcal{O}\left(h^{-N_{0}}\right) .
$$

This leads to the existence of $C_{1}=C_{1}(N)>0$ such that for $h$ small enough:

$$
\begin{aligned}
C_{1} h^{-N_{0}}\left\|\tilde{w}_{h}\right\|_{H^{1}\left(V_{\eta}\right)} \geq & \left\|h d \tilde{w}_{h}\right\|_{L^{2}\left(V_{\eta}\right)}^{2}+\left\|h d^{*} \tilde{w}_{h}\right\|_{L^{2}\left(V_{\eta}\right)}^{2}-h \int_{\Gamma_{1}}\left\langle\tilde{w}_{h}, \tilde{w}_{h}\right\rangle_{T_{\sigma}^{*} \Omega} \partial_{n} f d \sigma \\
& +\left\langle\left(|\nabla f|^{2}-\left|\nabla \varphi_{N}\right|^{2}+h \mathcal{L}_{\nabla f}+h \mathcal{L}_{\nabla f}^{*}\right) \tilde{w}_{h}, \tilde{w}_{h}\right\rangle_{L^{2}\left(V_{\eta}\right)} .
\end{aligned}
$$

Since $\varphi_{N} \leq \varphi+N h \ln \frac{1}{h}, \varphi \leq C h$ on $\Omega_{-}$and $\left\|w_{h}\right\|_{H^{1}\left(V_{\eta}\right)}=\mathcal{O}\left(h^{\infty}\right)($ see 197) $)$

$$
\left\|\tilde{w}_{h}\right\|_{L^{2}\left(\Omega_{-}\right)} \leq e^{C} h^{-N}\left\|w_{h}\right\|_{L^{2}\left(\Omega_{-}\right)} \leq C_{2}(C, N) .
$$

Thus, since $\mathcal{L}_{\nabla f}+\mathcal{L}_{\nabla f}^{*}$ is a $0^{\text {th }}$ order differential operator, we get the existence of $C_{3}>0$ independent of $(C, N)$ and of $C_{4}=C_{4}(C, N)$ such that:

$$
\begin{aligned}
C_{4}\left(h^{-N_{0}}\left\|\tilde{w}_{h}\right\|_{H^{1}\left(V_{\eta}\right)}+1\right) \geq & \left\|h d \tilde{w}_{h}\right\|_{L^{2}\left(V_{\eta}\right)}^{2}+\left\|h d^{*} \tilde{w}_{h}\right\|_{L^{2}\left(V_{\eta}\right)}^{2}-h \int_{\Gamma_{1}}\left\langle\tilde{w}_{h}, \tilde{w}_{h}\right\rangle_{T_{\sigma}^{*} \Omega} \partial_{n} f d \sigma \\
& +\left\langle\left|\nabla f_{-}\right|^{2} \tilde{w}_{h}, \tilde{w}_{h}\right\rangle_{L^{2}\left(V_{\eta}\right)}+\left\langle\left(\left|\nabla f_{+}\right|^{2}-\left|\nabla \varphi_{N}\right|^{2}-C_{3} h\right) \tilde{w}_{h}, \tilde{w}_{h}\right\rangle_{L^{2}\left(\Omega_{+}\right)} .
\end{aligned}
$$

Moreover, by definition of $\varphi_{N}$, a.e. in $\Omega_{+}, \nabla \varphi_{N}=\nabla \psi 1_{\left\{\varphi_{N}=\psi\right\}}+\nabla f_{+}\left(1-\frac{C h}{f_{+}}\right) 1_{\left\{\varphi_{N}<\psi\right\}}$. Now, 
- On $\left\{\varphi_{N}=\psi\right\}$, since by 214 $\left\{\varphi_{N}=\psi\right\}$ avoids a neighborhood of $\left\{\left(z, x_{d}\right), x_{d} \in\right.$ $\left.\operatorname{supp} \eta_{d}\right\}=\left\{x \in V_{\eta},\left|\nabla f_{+}(x)\right|=0\right\}$ (see (140)), we get

$$
\left|\nabla f_{+}\right|^{2}-\left|\nabla \varphi_{N}\right|^{2} \geq\left(1-M^{2}(1-\varepsilon)^{2}\right)\left|\nabla f_{+}\right|^{2} \geq c_{\varepsilon}>0,
$$

where (216) have been used;

- On $\left\{\varphi_{N}<\psi\right\} \cap \Omega_{+}$, we get like in the proof of Proposition 87 (see 160) and 1162),

$$
\left|\nabla f_{+}\right|^{2}-\left|\nabla \varphi_{N}\right|^{2} \geq K C h
$$

Choosing $C>\max \left(1, \frac{C_{3}}{K}\right)$, we obtain that for $h$ small enough:

$$
\begin{gathered}
C_{4}\left(h^{-N_{0}}\left\|\tilde{w}_{h}\right\|_{H^{1}\left(V_{\eta}\right)}+1\right) \geq\left\|h d \tilde{w}_{h}\right\|_{L^{2}\left(V_{\eta}\right)}^{2}+\left\|h d^{*} \tilde{w}_{h}\right\|_{L^{2}\left(V_{\eta}\right)}^{2}-h \int_{\Gamma_{1}}\left\langle\tilde{w}_{h}, \tilde{w}_{h}\right\rangle_{T_{\sigma}^{*} \Omega} \partial_{n} f d \sigma \\
\quad+\left\langle\left|\nabla f_{-}\right|^{2} \tilde{w}_{h}, \tilde{w}_{h}\right\rangle_{L^{2}\left(V_{\eta}\right)}+\left(K C-C_{3}\right) h\left(\left\|\tilde{w}_{h}\right\|_{L^{2}\left(V_{\eta}\right)}^{2}-\left\|\tilde{w}_{h}\right\|_{L^{2}\left(\Omega_{-}\right)}^{2}\right) \cdot
\end{gathered}
$$

We can now control from below the r.h.s. of the above estimate exactly as we did at the end of the first step of Proposition 87; defining $C_{5}(C):=\frac{K C-C_{3}}{2\left\|\left.|| \nabla f_{-}\right|^{2}\right\|_{L}{ }^{\infty}\left(V_{\eta}\right)}$ (see (138)), one gets the inequality

$$
\left(K C-C_{3}\right) h\left\|\tilde{w}_{h}\right\|_{L^{2}\left(V_{\eta}\right)}^{2}+\left\langle\left|\nabla f_{-}\right|^{2} \tilde{w}_{h}, \tilde{w}_{h}\right\rangle_{L^{2}\left(V_{\eta}\right)} \geq\left(1+2 C_{5} h\right)\left\langle\left|\nabla f_{-}\right|^{2} \tilde{w}_{h}, \tilde{w}_{h}\right\rangle_{L^{2}\left(V_{\eta}\right)}
$$

and from Lemma 76 applied with $u=\tilde{w}_{h}, f=-\tilde{\eta} f_{-}$where $\tilde{\eta} \in C^{\infty}(\bar{\Omega},[0,1]), \tilde{\eta}=1$ on $\operatorname{supp} \eta, \operatorname{supp} \tilde{\eta} \subset(\operatorname{supp} \eta+B(0, \alpha)) \cap \bar{\Omega}$ for $\alpha>0$ such that $f_{-}$is smooth on supp $\tilde{\eta}$ and $\frac{h}{1+C_{5} h}$ instead of $h$, one gets the following lower bound:

$$
\begin{aligned}
-h \int_{\Gamma_{1}}\left\langle\tilde{w}_{h}, \tilde{w}_{h}\right\rangle \partial_{n} f d \sigma= & h \int_{\Gamma_{1}}\left\langle\tilde{w}_{h}, \tilde{w}_{h}\right\rangle \partial_{n} f-d \sigma \\
\geq & -\left(1+C_{5} h\right)\left\|\left|\nabla f_{-}\right| \tilde{w}_{h}\right\|_{L^{2}\left(V_{\eta}\right)}^{2} \\
& -\frac{h^{2}}{1+C_{5} h}\left(\left\|d \tilde{w}_{h}\right\|_{L^{2}\left(V_{\eta}\right)}^{2}+\left\|d^{*} \tilde{w}_{h}\right\|_{L^{2}\left(V_{\eta}\right)}^{2}\right)-C_{6} h\left\|\tilde{w}_{h}\right\|_{L^{2}\left(V_{\eta}\right)}^{2},
\end{aligned}
$$

where $C_{6}$ is some positive constant independent of $C$ (it only depends on $f_{-}$). Injecting the estimates (225) and (226) in (224) then leads to:

$$
\begin{aligned}
C_{4}\left(h^{-N_{0}}\left\|\tilde{w}_{h}\right\|_{H^{1}\left(V_{\eta}\right)}+1\right) \geq \frac{C_{5} h^{3}}{1+C_{5} h}\left(\left\|d \tilde{w}_{h}\right\|_{L^{2}\left(V_{\eta}\right)}^{2}+\left\|d^{*} \tilde{w}_{h}\right\|_{L^{2}\left(V_{\eta}\right)}^{2}\right) \\
+C_{5} h\left\|\left|\nabla f_{-}\right| \tilde{w}_{h}\right\|_{L^{2}\left(V_{\eta}\right)}^{2}-\left(K C-C_{3}\right) h\left\|\tilde{w}_{h}\right\|_{L^{2}\left(\Omega_{-}\right)}^{2}-C_{6} h\left\|\tilde{w}_{h}\right\|_{L^{2}\left(V_{\eta}\right)}^{2} .
\end{aligned}
$$

Then, since $\left|\nabla f_{-}\right| \geq c>0$ on $\overline{V_{\eta}}$ (see $(138)$ ), $\lim _{C \rightarrow \infty} C_{5}(C)=+\infty$. Therefore, since $C_{6}$ is independent of $C$, one can choose $C$ such that $c^{2} C_{5}-C_{6}>0$, which implies, remembering also $\left\|\tilde{w}_{h}\right\|_{L^{2}\left(\Omega_{-}\right)} \leq C_{2}(C, N)$ (see 223$)$ ), the existence of a constant $C_{7}>0$ and a constant $h_{0}>0$ such that, for every $h \in\left(0, h_{0}\right)$,

$$
\left\|\tilde{w}_{h}\right\|_{L^{2}\left(V_{\eta}\right)}^{2}+\left\|d \tilde{w}_{h}\right\|_{L^{2}\left(V_{\eta}\right)}^{2}+\left\|d^{*} \tilde{w}_{h}\right\|_{L^{2}\left(V_{\eta}\right)}^{2} \leq \frac{C_{7}}{h^{3}}\left(h^{-N_{0}}\left\|\tilde{w}_{h}\right\|_{H^{1}\left(V_{\eta}\right)}+1\right) .
$$

According to Gaffney's inequality (121), this finally leads to the existence of a positive constant $C_{8}$ such that

$$
\left\|\tilde{w}_{h}\right\|_{H^{1}\left(V_{\eta}\right)} \leq C_{8} h^{-N_{0}-3}
$$


Moreover, according to 214, we have $\varphi_{N}=\varphi+N h \ln \frac{1}{h}$ in $V_{\eta} \cap\left\{x^{\prime} \in \overline{\Gamma_{S t}^{\prime}}\right\}$ and then $\varphi_{N}-N h \ln \frac{1}{h}-f_{+} \geq-C_{9} h \ln \frac{1}{h}$ (with a constant $C_{9}$ independent of $N$ ) in $V_{\Gamma_{S t}^{\prime}} \subset$ $V_{\eta} \cap\left\{x^{\prime} \in \overline{\Gamma_{S t}^{\prime}}\right\}$. Therefore, there exists $N_{1}$ independent of $N$ such that for $h$ small enough,

$$
\left\|e^{\frac{f_{+}}{h}} w_{h}\right\|_{H^{1}\left(V_{\Gamma_{S t}^{\prime}}\right)} \leq C_{N} h^{N-N_{1}},
$$

from which 208 and 209) follow since $N$ is arbitrary.

Step 2: Comparison in $V_{\Gamma_{S t}}$.

We work now with the cut-off function $\chi$ defined in (194). Recall that $\eta \chi=\chi$. Similarly as in the previous step, let us define the sets

$$
\Omega_{-}=\left\{x \in V_{\Gamma_{S t}^{\prime}} \text { s.t. } \Psi(x) \leq C h\right\} \text { and } \Omega_{+}=V_{\Gamma_{S t}^{\prime}} \backslash \Omega_{-},
$$

and the function

$$
\varphi_{N}=\min \left\{\varphi+N h \ln \frac{1}{h}, \psi\right\}
$$

where $\varphi$ and $\psi$ are respectively defined by

$$
\varphi=\left\{\begin{array}{l}
\Psi-C h \ln \frac{\Psi}{h} \text { if } \Psi>C h \\
\Psi-C h \ln C \text { if } \Psi \leq C h
\end{array}\right.
$$

and

$$
\psi(x)=\min \left\{\varphi(y)+(1-\varepsilon) d_{a}(x, y), y \in \operatorname{supp} \nabla \chi\right\} .
$$

The constant $C>1$ will be chosen at the end of the proof. Following the proof of 214, there exists $\varepsilon \in(0,1)$ such that for any $h \in\left(0, h_{0}\right)$ with $h_{0}=h_{0}(N, \varepsilon)$ small enough,

$$
\varphi_{N}=\varphi+N h \ln \frac{1}{h}<\psi \text { in } V_{\Gamma_{S t}} .
$$

Indeed, using the fact that $\Psi(x)=d_{a}(x, z)$ and a triangular inequality,

$$
\forall(x, y) \in \overline{V_{\Gamma_{S t}}} \times \operatorname{supp} \nabla \chi, \quad \Psi(x)<\Psi(y)+d_{a}(x, y) .
$$

The inequality is strict since if $\Psi(x)=\Psi(y)+d_{a}(x, y)$ for some $(x, y) \in \overline{V_{\Gamma_{S t}}} \times \operatorname{supp} \nabla \chi$, then $\Phi(x)-\Phi(y)=d_{a}(x, y)$ and from Corollary 72 , up to modifying $\eta$ such that $V_{\eta} \subset V_{\alpha}$ (see Corollary 72 for the definition of $V_{\alpha}$ ) there exists a generalized integral curve (in the sense of Definition 65 of $\left\{\begin{array}{c}-\nabla \Phi \text { on } V_{\alpha} \cap \Omega \\ -\nabla_{T} \Phi \text { on } \partial \Omega\end{array}\right.$ joining $x \in \overline{V_{\Gamma_{S t}}}$ to $y \notin \overline{V_{\Gamma_{S t}}}$. This contradicts the fact that $V_{\Gamma_{S t}}$ is stable for (193). The end of the proof of 227) then follows exactly the same lines of the proof of (214).

Moreover, owing to the properties of $d_{a}$, one has analogously to 218 the following estimate valid a.e. in $V_{\Gamma_{S t}^{\prime}}$ :

$$
|\nabla \psi| \leq(1-\varepsilon)|\nabla f|=(1-\varepsilon)|\nabla \Psi| .
$$


Let us finally mention the following inequalities, valid for $h \in\left(0, h_{0}\right)$ with $h_{0}=$ $h_{0}(N, \varepsilon)>0$ small enough:

$$
\begin{aligned}
\varphi_{N} & \leq \Psi+N h \ln \frac{1}{h} \quad \text { in } V_{\Gamma_{S t}^{\prime}} \\
\text { and } \varphi_{N} & =\psi \leq \varphi \leq \Psi \quad \text { on } \operatorname{supp} \nabla \chi .
\end{aligned}
$$

We are now in position to prove (207). Let us define

$$
\tilde{w}_{h}=e^{\frac{\varphi_{N}}{h}} w_{h}=e^{\frac{\varphi_{N}}{h}} \chi\left(u_{h}^{(1)}-c_{z}(h) u_{z, w k b}^{(1)}\right) .
$$

Using the relations 202 -206 and the integration by parts formulae (158) and (120), there exists $C_{1}>0$ (only depending on $f$ ) such that

$$
\begin{gathered}
\left\|\left(r_{1}+r_{1}^{\prime}\right) e^{\frac{\varphi_{N}-\Psi}{h}}\right\|_{L^{2}\left(V_{\Gamma_{S t}^{\prime}}\right)}\left\|\tilde{w}_{h}\right\|_{L^{2}\left(V_{\Gamma_{S t}^{\prime}}\right)}+\left\|\left(r_{2}+r_{2}^{\prime}\right) e^{\frac{\varphi_{N}-\Psi}{h}}\right\|_{L^{2}\left(\Gamma_{1}\right)}\left\|\tilde{w}_{h}\right\|_{L^{2}\left(\Gamma_{1}\right)} \\
+C_{1} h \int_{\Gamma_{1}}\left\langle\tilde{w}_{h}, \tilde{w}_{h}\right\rangle_{T_{\sigma}^{*} \Omega} d \sigma \geq\left\|h d \tilde{w}_{h}\right\|_{L^{2}\left(V_{\Gamma_{S t}^{\prime}}\right)}^{2}+\left\|h d^{*} \tilde{w}_{h}\right\|_{L^{2}\left(V_{\Gamma_{S t}^{\prime}}\right)}^{2} \\
+\left\langle\left(|\nabla f|^{2}-\left|\nabla \varphi_{N}\right|^{2}-C_{1} h\right) \tilde{w}_{h}, \tilde{w}_{h}\right\rangle_{L^{2}\left(\Omega_{+}\right)}-C_{1} h\left\|\tilde{w}_{h}\right\|_{L^{2}\left(\Omega_{-}\right)}^{2},
\end{gathered}
$$

where we have used the fact that almost everywhere on $\Omega_{-},\left|\nabla \varphi_{N}\right|$ is either equal to $|\nabla \varphi|=|\nabla \Psi|=|\nabla f|$ or to $|\nabla \psi| \leq(1-\varepsilon)|\nabla f|$. Moreover, since $\varphi_{N}-\Psi \leq N h \ln \frac{1}{h}$ (see 229), one has from 203 and 205)

$$
\left\|r_{1} e^{\frac{\varphi_{N}-\Psi}{h}}\right\|_{L^{2}\left(V_{\Gamma_{S t}^{\prime}}\right)}+\left\|r_{2} e^{\frac{\varphi_{N}-\Psi}{h}}\right\|_{L^{2}\left(\Gamma_{1}\right)}=\mathcal{O}\left(h^{\infty}\right)
$$

and, since $\varphi_{N} \leq \Psi$ on $\operatorname{supp} \nabla \chi($ see 230$)$, one gets from 204 and 206

$$
\left\|r_{1}^{\prime} e^{\frac{\varphi_{N}-\Psi}{h}}\right\|_{L^{2}\left(V_{\Gamma_{S t}^{\prime}}\right)}+\left\|r_{2}^{\prime} e^{\frac{\varphi_{N}-\Psi}{h}}\right\|_{L^{2}\left(\Gamma_{1}\right)}=\mathcal{O}\left(h^{-N_{0}}\right) .
$$

Additionally, since $\Psi=f-f(z)$ on $\Gamma_{1}$ and $\varphi_{N}-\Psi \leq N h \ln \frac{1}{h}$ (see 229$)$ ), we deduce from the relation (209) obtained in the first step the following estimate:

$$
\left\|\tilde{w}_{h}\right\|_{L^{2}\left(\Gamma_{1}\right)}=\left\|e^{\frac{\varphi_{N}}{h}} \chi\left(u_{h}^{(1)}-c_{z}(h) u_{z, w k b}^{(1)}\right)\right\|_{L^{2}\left(\Gamma_{S t}^{\prime}\right)}=\mathcal{O}\left(h^{\infty}\right) .
$$

Consequently, using in addition the relation

$$
\left\|\tilde{w}_{h}\right\|_{L^{2}\left(\Omega_{-}\right)} \leq e^{C} h^{-N}\left\|w_{h}\right\|_{L^{2}\left(\Omega_{-}\right)} \leq C_{2}(C, N),
$$

(since $\varphi_{N} \leq \varphi+N h \ln \frac{1}{h}, \varphi \leq C h$ on $\Omega_{-}$and $\left\|w_{h}\right\|_{L^{2}\left(V_{\Gamma_{S t}^{\prime}}\right)}=O\left(h^{\infty}\right)$ from 197)), we deduce from (231) the existence of some positive constant $C_{3}=C_{3}\left(C, C_{1}, N\right)$ such that

$$
\begin{aligned}
C_{3}\left(h^{-N_{0}}\left\|\tilde{w}_{h}\right\|_{L^{2}\left(V_{\Gamma_{S t}^{\prime}}\right)}+1\right) \geq & \left\|h d \tilde{w}_{h}\right\|_{L^{2}\left(V_{\Gamma_{S t}^{\prime}}\right)}^{2}+\left\|h d^{*} \tilde{w}_{h}\right\|_{L^{2}\left(V_{\Gamma_{S t}^{\prime}}\right)}^{2} \\
& +\left\langle\left(|\nabla f|^{2}-\left|\nabla \varphi_{N}\right|^{2}-C_{1} h\right) \tilde{w}_{h}, \tilde{w}_{h}\right\rangle_{L^{2}\left(\Omega_{+}\right)} .
\end{aligned}
$$

Lastly, one has a.e. in $\Omega_{+}, \nabla \varphi_{N}=\nabla \psi 1_{\left\{\varphi_{N}=\psi\right\}}+\nabla \Psi\left(1-\frac{C h}{\Psi}\right) 1_{\left\{\varphi_{N}<\psi\right\}}$, and thus

- on $\left\{\varphi_{N}=\psi\right\}$, from 228,

$$
|\nabla f|^{2}-\left|\nabla \varphi_{N}\right|^{2} \geq\left(2 \varepsilon-\varepsilon^{2}\right)|\nabla f|^{2} \geq c_{\varepsilon}>0,
$$


- on $\left\{\varphi_{N}<\psi\right\} \cap \Omega_{+}$, there exists $C_{4}>0$ independent of $C$ such that,

$$
|\nabla f|^{2}-\left|\nabla \varphi_{N}\right|^{2} \geq C h \frac{|\nabla f|^{2}}{\Psi} \geq C_{4} C h .
$$

Taking $C>\frac{C_{1}}{C_{4}}$ and adding $\left(C C_{4}-C_{1}\right) h\left\|\tilde{w}_{h}\right\|_{L^{2}\left(\Omega_{-}\right)}^{2}$ to 232 then leads to $C_{5}\left(h^{-N_{0}}\left\|\tilde{w}_{h}\right\|_{L^{2}\left(V_{\Gamma_{S t}^{\prime}}\right)}+1\right) \geq\left\|h d \tilde{w}_{h}\right\|_{L^{2}\left(V_{\Gamma_{S t}^{\prime}}\right)}^{2}+\left\|h d^{*} \tilde{w}_{h}\right\|_{L^{2}\left(V_{\Gamma_{S t}^{\prime}}\right)}^{2}+\left(C C_{4}-C_{1}\right) h\left\|\tilde{w}_{h}\right\|_{L^{2}\left(V_{\Gamma_{S t}^{\prime}}\right)}^{2}$,

for a constant $C_{5}$ depending on $C$ and $N$. Using Gaffney's inequality (121), we consequently get the existence of $C_{6}>0$ such that

$$
\left\|\tilde{w}_{h}\right\|_{H^{1}\left(V_{\Gamma_{S t}^{\prime}}\right)} \leq C_{6} h^{-N_{0}-3 / 2}
$$

Now, since $\varphi_{N}-N h \ln \frac{1}{h}-\Psi \geq-C_{7} h \ln \frac{1}{h}$ in $V_{\Gamma_{S t}}$ (with a constant $C_{7}$ independent of $N$, from the definition of $\varphi$ and the fact that $\varphi_{N}-N h \ln \frac{1}{h}=\varphi$ in $V_{\Gamma_{S t}}$, see (227)), we also get the existence of $N_{2}$ independent of $N$ such that for $h$ small enough,

$$
\left\|e^{\frac{\Psi}{h}} w_{h}\right\|_{H^{1}\left(V_{\Gamma_{S t}}\right)} \leq C_{N} h^{N-N_{2}}
$$

for some constant $C_{N}>0$, which concludes the proof of Proposition 91 .

\subsection{Proof of Theorem 1}

The aim of this section is to conclude the proof of Theorem 1 by checking that the function $\tilde{u}$ and the family of 1 -forms $\left(\tilde{\phi}_{i}\right)_{i=1, \ldots, n}$ introduced in Section 4.2 satisfy the estimates appearing in Proposition 25 rewritten in the flat space (see Section 2.2.2). In all this section, we assume in addition to the hypotheses [H1], [H2] and [H3], that 200 and (21) hold.

From Sections 4.2.1 and 4.2.2, it only remains to prove (56), (57), (58) and (59). Let us start with a lemma about the normalisation term appearing in 156 .

Lemma 92. Let us assume that the hypotheses [H1], [H2] and [H3] hold. Let us define $\Theta_{i}:=\sqrt{\int_{\Omega}\left|\chi_{i}(x) u_{h, i}^{(1)}(x)\right|^{2} d x}$. There exist $c>0$ and $h_{0}>0$ such that for all $h \in\left(0, h_{0}\right)$,

$$
\Theta_{i}^{2}=1+O\left(e^{-\frac{c}{h}}\right)
$$

Proof. On the one hand, one has the upper bound

$$
\left\|\chi_{i} u_{h, i}^{(1)}\right\|_{L^{2}(\Omega)}=\left\|\chi_{i} u_{h, i}^{(1)}\right\|_{L^{2}\left(\dot{\Omega}_{i}\right)} \leq\left\|u_{h, i}^{(1)}\right\|_{L^{2}\left(\dot{\Omega}_{i}\right)}=1 .
$$

On the other hand, the triangular inequality yields the lower bound

$$
\left\|\chi_{i} u_{h, i}^{(1)}\right\|_{L^{2}\left(\dot{\Omega}_{i}\right)} \geq\left\|u_{h, i}^{(1)}\right\|_{L^{2}\left(\dot{\Omega}_{i}\right)}-\left\|\left(1-\chi_{i}\right) u_{h, i}^{(1)}\right\|_{L^{2}\left(\dot{\Omega}_{i}\right)}=1-\left\|\left(1-\chi_{i}\right) u_{h, i}^{(1)}\right\|_{L^{2}\left(\dot{\Omega}_{i}\right)} .
$$


Thanks to Proposition 87, there exist $N \in \mathbb{N}$ and $c>0$ independent of $h$ such that

$$
\begin{aligned}
\left\|\left(1-\chi_{i}\right) u_{h, i}^{(1)}\right\|_{L^{2}\left(\dot{\Omega}_{i}\right)}^{2} & =\int_{\dot{\Omega}_{i}}\left|\left(1-\chi_{i}(x)\right) u_{h, i}^{(1)}(x) e^{\frac{1}{h} d_{a}\left(x, z_{i}\right)} e^{-\frac{1}{h} d_{a}\left(x, z_{i}\right)}\right|^{2} d x \\
& \leq \int_{\dot{\Omega}_{i} \backslash \mathcal{V}_{i}}\left|u_{h, i}^{(1)}(x) e^{\frac{1}{h} d_{a}\left(x, z_{i}\right)} e^{-\frac{1}{h} d_{a}\left(x, z_{i}\right)}\right|^{2} d x \\
& \leq C h^{-N} e^{-\inf _{\dot{\Omega}_{i} \backslash \mathcal{V}_{i}} \frac{2}{h} d_{a}\left(., z_{i}\right)} \leq C e^{-\frac{c}{h}},
\end{aligned}
$$

where, we recall $\mathcal{V}_{i}=\left\{x \in \Omega, \chi_{i}=1\right\}$. This concludes the proof of Lemma 92 .

We are now in position to check the estimates stated in Section 2.2.2.

Step 1. Study of the term $\left\|\left(1-\pi_{\left[0, h^{\frac{3}{2}}\right)}\left(\Delta_{f, h}^{D,(1)}(\Omega)\right)\right) \tilde{\phi}_{i}\right\|_{H^{1}(\Omega)}$.

We recall that from (121), $\tilde{\phi}_{i}$ belongs to $\Lambda^{1} H_{T}^{1}(\Omega)$ and then we get from Lemma 23 that there exist $c>0$ and $h_{0}>0$ such that for all $h \in\left(0, h_{0}\right)$,

$$
\begin{aligned}
\left\|\left(1-\pi_{\left[0, h^{\frac{3}{2}}\right)}\left(\Delta_{f, h}^{D,(1)}(\Omega)\right)\right) \tilde{\phi}_{i}\right\|_{L^{2}(\Omega)}^{2} & \leq c h^{-3 / 2}\left(\left\|d_{f, h} \tilde{\phi}_{i}\right\|_{L^{2}(\Omega)}^{2}+\left\|d_{f, h}^{*} \tilde{\phi}_{i}\right\|_{L^{2}(\Omega)}^{2}\right) \\
& =c h^{-3 / 2}\left(\left\|d_{f, h} \tilde{\phi}_{i}\right\|_{L^{2}\left(\dot{\Omega}_{i}\right)}^{2}+\left\|d_{f, h}^{*} \tilde{\phi}_{i}\right\|_{L^{2}\left(\dot{\Omega}_{i}\right)}^{2}\right) .
\end{aligned}
$$

Moreover, from Proposition 83 (items (ii) et $(i i i)$ )

$$
d_{f, h} \tilde{\phi}_{i}=\Theta_{i}^{-1}\left(\chi_{i} d_{f, h} u_{h, i}^{(1)}+h d \chi_{i} \wedge u_{h, i}^{(1)}\right)=\Theta_{i}^{-1} h d \chi_{i} \wedge u_{h, i}^{(1)}
$$

and

$$
d_{f, h}^{*} \tilde{\phi}_{i}=\Theta_{i}^{-1}\left(\chi_{i} d_{f, h}^{*} u_{h, i}^{(1)}-h u_{h, i}^{(1)} \cdot \nabla \chi_{i}\right)=-\Theta_{i}^{-1} h u_{h, i}^{(1)} \cdot \nabla \chi_{i} .
$$

As a consequence, using Lemma 92 and Proposition 87 , one gets for some $N \in \mathbb{N}$ and for some $c>0$ which may change from one occurrence to another,

$$
\begin{aligned}
\left\|\left(1-\pi_{\left[0, h^{\frac{3}{2}}\right)}\left(\Delta_{f, h}^{D,(1)}(\Omega)\right)\right) \tilde{\phi}_{i}\right\|_{L^{2}(\Omega)}^{2} & \leq c h^{-3 / 2}\left(\left\|h d \chi_{i} \wedge u_{h, i}^{(1)}\right\|_{L^{2}\left(\dot{\Omega}_{i}\right)}^{2}+\left\|h u_{h, i}^{(1)} \cdot \nabla \chi_{i}\right\|_{L^{2}\left(\dot{\Omega}_{i}\right)}^{2}\right) \\
& \leq c h^{1 / 2} \int_{\operatorname{supp} \nabla \chi_{i}}\left|u_{h, i}^{(1)}(x) e^{\frac{1}{h} d_{a}\left(x, z_{i}\right)} e^{-\frac{1}{h} d_{a}\left(x, z_{i}\right)}\right|^{2} d x \\
& \leq c h^{1 / 2-N} e^{-\inf _{\operatorname{supp} \nabla \chi_{i}} \frac{2}{h} d_{a}\left(\cdot, z_{i}\right)}
\end{aligned}
$$

The function $\chi_{i}$ can be chosen such that the set $\left\{x \in \dot{\Omega}_{i}, \nabla \chi_{i}(x) \neq 0\right\}$ is as close as needed to $\Gamma_{2, i}$ and to $\Gamma_{0}$ (see Figure 15 . Therefore by continuity of the Agmon distance, using (144)-145), for any $\delta>0$, one can choose $\chi_{i}$ satisfying the three conditions stated in Definition 86 and such that

$$
\inf _{z \in \operatorname{supp} \nabla \chi_{i}} d_{a}\left(z, z_{i}\right) \geq \min \left(d_{a}\left(x_{0}, z_{i}\right), \inf _{z \in B_{z_{i}}^{c}} d_{a}\left(z, z_{i}\right)\right)-\delta .
$$

From (20), there exists $r>0$ such that

$$
\inf _{z \in B_{z_{i}}^{c}} d_{a}\left(z, z_{i}\right) \geq \max \left[f\left(z_{n}\right)-f\left(z_{i}\right), f\left(z_{i}\right)-f\left(z_{1}\right)\right]+r
$$


In addition, using (21), there exists $r^{\prime}>0$ such that

$$
\begin{aligned}
d_{a}\left(z_{i}, x_{0}\right) & \geq f\left(z_{i}\right)-f\left(x_{0}\right) \geq f\left(z_{1}\right)-f\left(x_{0}\right) \geq f\left(z_{n}\right)-f\left(z_{1}\right)+r^{\prime} \\
& \geq \max \left[f\left(z_{n}\right)-f\left(z_{i}\right), f\left(z_{i}\right)-f\left(z_{1}\right)\right]+r^{\prime} .
\end{aligned}
$$

Therefore, choosing $\chi_{i}$ such that $\delta<\min \left(r, r^{\prime}\right)$, there exists $\varepsilon^{\prime}>0$ such that

$$
\inf _{z \in \operatorname{supp} \nabla \chi_{i}} d_{a}\left(z, z_{i}\right) \geq \max \left[f\left(z_{n}\right)-f\left(z_{i}\right), f\left(z_{i}\right)-f\left(z_{1}\right)\right]+\varepsilon^{\prime} .
$$

Using the estimate (236) in 234, there exist $\varepsilon_{1}>0, c>0, N \in \mathbb{N}$ and $h_{0}>0$, such that for every $h \in\left(0, h_{0}\right)$

$$
\begin{aligned}
\left\|\left(1-\pi_{\left[0, h^{\frac{3}{2}}\right)}\left(\Delta_{f, h}^{D,(1)}(\Omega)\right)\right) \tilde{\phi}_{i}\right\|_{L^{2}(\Omega)}^{2} & \leq c h^{-N} e^{-\frac{2}{h}\left(\max \left[f\left(z_{n}\right)-f\left(z_{i}\right), f\left(z_{i}\right)-f\left(z_{1}\right)\right]+\varepsilon^{\prime}\right)} \\
& \leq e^{-\frac{2}{h}\left(\max \left[f\left(z_{n}\right)-f\left(z_{i}\right), f\left(z_{i}\right)-f\left(z_{1}\right)\right]+\varepsilon_{1}\right)}
\end{aligned}
$$

This last inequality leads to the desired estimate in the $L^{2}(\Omega)$-norm. In order to get the same upper bound in the $H^{1}(\Omega)$-norm, notice now that one has

$$
\begin{aligned}
& \left(1-\pi_{\left[0, h^{\frac{3}{2}}\right)}\left(\Delta_{f, h}^{D,(2)}(\Omega)\right)\right) d_{f, h} \tilde{\phi}_{i}=d_{f, h}\left(1-\pi_{\left[0, h^{\frac{3}{2}}\right.}\left(\Delta_{f, h}^{(D, 1)}(\Omega)\right)\right) \tilde{\phi}_{i} \\
& =h d\left(1-\pi_{\left[0, h^{\frac{3}{2}}\right)}\left(\Delta_{f, h}^{D,(1)}(\Omega)\right)\right) \tilde{\phi}_{i}+d f \wedge\left(1-\pi_{\left[0, h^{\frac{3}{2}}\right)}\left(\Delta_{f, h}^{D,(1)}(\Omega)\right)\right) \tilde{\phi}_{i} .
\end{aligned}
$$

Therefore it holds

$$
\begin{aligned}
h d\left(1-\pi_{\left[0, h^{\frac{3}{2}}\right)}\left(\Delta_{f, h}^{D,(1)}(\Omega)\right)\right) \tilde{\phi}_{i}= & \left(1-\pi_{\left[0, h^{\frac{3}{2}}\right)}\left(\Delta_{f, h}^{D,(2)}(\Omega)\right)\right) d_{f, h} \tilde{\phi}_{i} \\
& -d f \wedge\left(1-\pi_{\left[0, h^{\frac{3}{2}}\right)}\left(\Delta_{f, h}^{D,(1)}(\Omega)\right)\right) \tilde{\phi}_{i} .
\end{aligned}
$$

Let us introduce $K_{i}:=\max \left[f\left(z_{n}\right)-f\left(z_{i}\right), f\left(z_{i}\right)-f\left(z_{1}\right)\right]$. From (237), there exist $C>0$ and $h_{0}>0$, such that for all $h \in\left(0, h_{0}\right)$

$$
\left\|d f \wedge\left(1-\pi_{\left[0, h^{\frac{3}{2}}\right)}\left(\Delta_{f, h}^{D,(1)}(\Omega)\right)\right) \tilde{\phi}_{i}\right\|_{L^{2}(\Omega)}^{2} \leq C e^{-\frac{2}{h}\left(K_{i}+\varepsilon_{1}\right)} .
$$

Moreover, using (233) and 234 there exist $\varepsilon>0, C>0$ and $h_{0}>0$, such that for all $h \in\left(0, h_{0}\right)$,

$$
\begin{aligned}
\left\|\left(1-\pi_{\left[0, h^{\frac{3}{2}}\right)}\left(\Delta_{f, h}^{D,(2)}(\Omega)\right)\right) d_{f, h} \tilde{\phi}_{i}\right\|_{L^{2}(\Omega)}^{2} & \leq\left\|d_{f, h} \tilde{\phi}_{i}\right\|_{L^{2}(\Omega)}^{2}=\Theta_{i}^{-2}\left\|h d \chi_{i} \wedge u_{h, i}^{(1)}\right\|_{L^{2}\left(\dot{\Omega}_{i}\right)}^{2} \\
& \leq C e^{-\frac{2}{h}\left(K_{i}+\varepsilon\right)} .
\end{aligned}
$$

Thus one gets: there exist $\varepsilon>0, C>0$ and $h_{0}>0$, such that for all $h \in\left(0, h_{0}\right)$,

$$
h^{2}\left\|d\left(1-\pi_{\left[0, h^{\frac{3}{2}}\right)}\left(\Delta_{f, h}^{D,(1)}(\Omega)\right)\right) \tilde{\phi}_{i}\right\|_{L^{2}(\Omega)}^{2} \leq C e^{-\frac{2}{h}\left(K_{i}+\varepsilon\right)} .
$$

Similarly, there exist $\varepsilon>0, C>0$ and $h_{0}>0$, such that for all $h \in\left(0, h_{0}\right)$

$$
h^{2}\left\|d^{*}\left(1-\pi_{\left[0, h^{\frac{3}{2}}\right)}\left(\Delta_{f, h}^{D,(1)}(\Omega)\right)\right) \tilde{\phi}_{i}\right\|_{L^{2}(\Omega)}^{2} \leq C e^{-\frac{2}{h}\left(K_{i}+\varepsilon\right)} .
$$


As a consequence, using (121), there exist $\varepsilon>0, C>0$ and $h_{0}>0$, such that for all $h \in\left(0, h_{0}\right)$

$$
\left\|\left(1-\pi_{\left[0, h^{\frac{3}{2}}\right)}\left(\Delta_{f, h}^{D,(1)}(\Omega)\right)\right) \tilde{\phi}_{i}\right\|_{H^{1}(\Omega)}^{2} \leq C e^{-\frac{2}{h}\left(K_{i}+\varepsilon\right)} .
$$

This concludes the proof of (56).

Step 2. Study of the terms $\int_{\Omega} \tilde{\phi}_{i} \cdot \tilde{\phi}_{j}$ for $(i, j) \in\{1, \ldots, n\}^{2}$.

Let $(i, j) \in\{1, \ldots, n\}^{2}$ be such that $i<j$. One then has $f\left(z_{i}\right) \leq f\left(z_{j}\right)$. From Proposition 66, it holds $d_{a}\left(z_{i}, z_{j}\right)>f\left(z_{j}\right)-f\left(z_{i}\right)$. Now, according to Proposition 87 and Lemma 92 and to the triangular inequality for $d_{a}$, there exist $\varepsilon>0, N \in \mathbb{N}$ and $h_{0}>0$ such that for all $h \in\left(0, h_{0}\right)$,

$$
\begin{aligned}
\left|\int_{\Omega} \tilde{\phi}_{i}(x) \cdot \tilde{\phi}_{j}(x) d x\right| & \leq e^{-\frac{d_{a}\left(z_{j}, z_{i}\right)}{h}} \int_{\operatorname{supp} \chi_{i} \cap \operatorname{supp} \chi_{j}} e^{\frac{d_{a}\left(x, z_{i}\right)}{h}}\left|\tilde{\phi}_{i}(x)\right| e^{\frac{d_{a}\left(x, z_{j}\right)}{h}}\left|\tilde{\phi}_{j}(x)\right| d x \\
& \leq \Theta_{i}^{-1} \Theta_{j}^{-1}\left\|e^{\frac{d_{a}\left(., z_{i}\right)}{h}} \chi_{i} u_{h, i}^{(1)}\right\|_{L^{2}\left(\dot{\Omega}_{i}\right)}\left\|e^{\frac{d_{a}\left(., z_{j}\right)}{h}} \chi_{j} u_{h, j}^{(1)}\right\|_{L^{2}\left(\dot{\Omega}_{j}\right)} e^{-\frac{d_{a}\left(z_{i}, z_{j}\right)}{h}} \\
& \leq C h^{-N} e^{-\frac{1}{h}\left(f\left(z_{j}\right)-f\left(z_{i}\right)+\varepsilon\right)} .
\end{aligned}
$$

This concludes the proof of (57).

Step 3. Study of the terms $\int_{\Sigma_{i}} \tilde{\phi}_{j} \cdot n e^{-\frac{f}{h}}$ for $(i, j) \in\{1, \ldots, n\}^{2}$.

By construction, for all $(i, j) \in\{1, \ldots, n\}^{2}$ such that $i \neq j$, one has

$$
\int_{\Sigma_{i}} \tilde{\phi}_{j} \cdot n e^{-\frac{1}{h} f}=0
$$

Now, let us compute the term $\int_{\Sigma_{i}} \tilde{\phi}_{i} \cdot n e^{-\frac{1}{h} f}$. Let $u_{z_{i}, w k b}^{(1)}$ be the WKB expansion defined by (184. Following the beginning of Section 4.4.2, let us consider

1. a neighborhood $V_{\Gamma_{S t, i}}$ of $\Sigma_{i}$ in $\bar{\Omega}$, which is stable under the dynamics 193 and such that, for some $\varepsilon>0, V_{\Gamma_{S t, i}}+B(0, \varepsilon) \subset V_{\Gamma_{1, i}} \cap\left(\Gamma_{1, i} \cup \dot{\Omega}_{i}\right)$

2. and a cut-off function $\chi_{w k b, i} \in C_{c}^{\infty}\left(\dot{\Omega}_{i} \cup \Gamma_{1, i}\right)$ with $\chi_{w k b, i} \equiv 1$ on a neighborhood of $\overline{V_{\Gamma_{S t, i}}}$ and such that $\operatorname{supp} \chi_{w k b, i} \subset V_{\Gamma_{1, i}} \cap\left(\dot{\Omega}_{i} \cup \Gamma_{1, i}\right)$.

Using Proposition 90 , there exists $c_{z_{i}}(h) \in \mathbb{R}_{+}^{*}$ such that

$$
\left\|\chi_{w k b, i}\left(u_{h, i}^{(1)}-c_{z_{i}}(h) u_{z_{i}, w k b}^{(1)}\right)\right\|_{H^{1}\left(\dot{\Omega}_{i}\right)}=O\left(h^{\infty}\right) .
$$

Let us now introduce

$$
\tilde{\phi}_{z_{i}, w k b}:=c_{z_{i}}(h) \chi_{w k b, i} u_{z_{i}, w k b}^{(1)}
$$

so that

$$
\int_{\Sigma_{i}} \tilde{\phi}_{i} \cdot n e^{-\frac{1}{h} f}=\int_{\Sigma_{i}} \tilde{\phi}_{z_{i}, w k b} \cdot n e^{-\frac{1}{h} f}+\int_{\Sigma_{i}}\left(\tilde{\phi}_{i}-\tilde{\phi}_{z_{i}, w k b}\right) \cdot n e^{-\frac{1}{h} f} .
$$


Let us first deal with the term $\int_{\Sigma_{i}} \tilde{\phi}_{z_{i}, w k b} \cdot n e^{-\frac{1}{h} f}$. Using Laplace's method (the computation is similar to (191)), one gets when $h \rightarrow 0$ (since $\Phi=f$ and $\partial_{n} \Phi=-\partial_{n} f$ on $\partial \Omega$, see (107) and (184))

$$
\begin{aligned}
\int_{\Sigma_{i}} \chi_{w k b, i} u_{z_{i}, w k b}^{(1)} \cdot n e^{-\frac{1}{h} f} & =\int_{\Sigma_{i}} e^{-\frac{\Phi-f\left(z_{i}\right)}{h}} a_{0} \partial_{n}(f-\Phi) e^{-\frac{1}{h} f}(1+O(h)) \\
& =2 e^{\frac{f\left(z_{i}\right)}{h}} \int_{\Sigma_{i}} e^{-\frac{2 f}{h}} a_{0} \partial_{n} f(1+O(h)) \\
& =\frac{2 \partial_{n} f\left(z_{i}\right) \pi^{\frac{d-1}{2}}}{\sqrt{\left.\operatorname{det} \operatorname{Hess} f\right|_{\partial \Omega}\left(z_{i}\right)}} h^{\frac{d-1}{2}} e^{-\frac{1}{h} f\left(z_{i}\right)}(1+O(h)) .
\end{aligned}
$$

Then one obtains when $h \rightarrow 0$

$$
\int_{\Sigma_{i}} \tilde{\phi}_{z_{i}, w k b} \cdot n e^{-\frac{1}{h} f}=c_{z_{i}}(h) \frac{2 \partial_{n} f\left(z_{i}\right) \pi^{\frac{d-1}{2}}}{\sqrt{\left.\operatorname{det} \operatorname{Hess} f\right|_{\partial \Omega}\left(z_{i}\right)}} h^{\frac{d-1}{2}} e^{-\frac{1}{h} f\left(z_{i}\right)}(1+O(h)) .
$$

We recall from Proposition 90 that in the limit $h \rightarrow 0$ :

$$
c_{z_{i}}(h)=C_{z_{i}, w k b}^{-1} h^{-\frac{d+1}{4}}\left(1+O\left(h^{\infty}\right)\right),
$$

where the constant $C_{z_{i}, w k b}$ is defined by (187). Therefore, in the limit $h \rightarrow 0$

$$
\int_{\Sigma_{i}} \tilde{\phi}_{z_{i}, w k b} \cdot n e^{-\frac{1}{h} f}=\frac{\pi^{\frac{d-1}{4}} \sqrt{2 \partial_{n} f\left(z_{i}\right)}}{\left(\left.\operatorname{det} \operatorname{Hess} f\right|_{\partial \Omega}\left(z_{i}\right)\right)^{1 / 4}} h^{\frac{d-3}{4}} e^{-\frac{1}{h} f\left(z_{i}\right)}(1+O(h)) .
$$

Let us now deal with the term $\int_{\Sigma_{i}}\left(\tilde{\phi}_{i}-\tilde{\phi}_{z_{i}, w k b}\right) \cdot n e^{-\frac{1}{h} f}$. One obtains using Lemmata 89 and 92 , that there exist $C>0, h_{0}>0$ and $\eta>0$ such that for all $h \in\left(0, h_{0}\right)$

$$
\begin{aligned}
\left|\int_{\Sigma_{i}}\left(\tilde{\phi}_{i}-\tilde{\phi}_{z_{i}, w k b}\right) \cdot n e^{-\frac{1}{h} f}\right|= & \left|\int_{\Sigma_{i}}\left(\frac{u_{h, i}^{(1)}}{\Theta_{i}}-c_{z_{i}}(h) u_{z_{i}, w k b}^{(1)}\right) \cdot n e^{-\frac{1}{h} f}\right| \\
= & \frac{1}{\Theta_{i}}\left|\int_{\Sigma_{i}}\left(u_{h, i}^{(1)}-\Theta_{i} c_{z_{i}}(h) u_{z_{i}, w k b}^{(1)}\right) \cdot n e^{-\frac{1}{h} f}\right| \\
\leq & \frac{e^{-\frac{1}{h} f\left(z_{i}\right)}}{\Theta_{i}} \int_{\Sigma_{i}}\left|\left(u_{h, i}^{(1)}-c_{z_{i}}(h) u_{z_{i}, w k b}^{(1)}\right) \cdot n\right| \\
& +e^{-\frac{1}{h} f\left(z_{i}\right)} \frac{\left|\Theta_{i}-1\right|}{\Theta_{i}}\left|c_{z_{i}}(h)\right| \int_{\Sigma_{i}}\left|u_{z_{i}, w k b}^{(1)} \cdot n\right| \\
\leq & C e^{-\frac{1}{h} f\left(z_{i}\right)}\left\|\chi_{w k b, i}\left(u_{h, i}^{(1)}-c_{z_{i}}(h) u_{z_{i}, w k b}^{(1)}\right)\right\|_{H^{1}\left(\dot{\Omega}_{i}\right)} \\
& +C e^{-\frac{1}{h} f\left(z_{i}\right)} e^{-\frac{\eta}{h}} h^{-\frac{d+1}{4}}\left\|\chi_{w k b, i} u_{z_{i}, w k b}^{(1)}\right\|_{H^{1}\left(\dot{\Omega}_{i}\right)}
\end{aligned}
$$

Therefore, one obtains using Proposition 90 and 188

$$
e^{\frac{1}{h} f\left(z_{i}\right)}\left|\int_{\Sigma_{i}}\left(\tilde{\phi}_{i}-\tilde{\phi}_{z_{i}, w k b}\right) \cdot n e^{-\frac{1}{h} f}\right|=O\left(h^{\infty}\right)+C e^{-\frac{\eta}{h}} h^{-\frac{d+5}{4}}=O\left(h^{\infty}\right) .
$$

In conclusion, we have when $h \rightarrow 0$

$$
\int_{\Sigma_{i}} \tilde{\phi}_{i} \cdot n e^{-\frac{1}{h} f}=\int_{\Sigma_{i}} \tilde{\phi}_{z_{i}, w k b} \cdot n e^{-\frac{1}{h} f}\left(1+O\left(h^{\infty}\right)\right),
$$


which gives the expected estimate

$$
\int_{\Sigma_{i}} \tilde{\phi}_{j} \cdot n e^{-\frac{1}{h} f}= \begin{cases}B_{i} h^{m} e^{-\frac{1}{h} f\left(z_{i}\right)}(1+O(h)) & \text { if } i=j \\ 0 & \text { if } i \neq j\end{cases}
$$

where

$$
m=\frac{d-3}{4} \quad \text { and } \quad B_{i}=\frac{\pi^{\frac{d-1}{4}} \sqrt{2 \partial_{n} f\left(z_{i}\right)}}{\left(\left.\operatorname{det} \operatorname{Hess} f\right|_{\partial \Omega}\left(z_{i}\right)\right)^{1 / 4}} .
$$

This concludes the proof of 59 .

Step 4. Study of the term $\int_{\Omega} \nabla \tilde{u} \cdot \tilde{\phi}_{i} e^{-\frac{1}{h} f}$.

First one has the equality by Definition 84 ,

$$
\int_{\Omega} \nabla \tilde{u} \cdot \tilde{\phi}_{i} e^{-\frac{1}{h} f}=\frac{\int_{\Omega} \nabla \chi \cdot \tilde{\phi}_{i} e^{-\frac{1}{h} f}}{\sqrt{\int_{\Omega} \chi^{2} e^{-\frac{2}{h} f}}},
$$

where $\nabla \chi \cdot \tilde{\phi}_{i}=\mathbf{i}_{\nabla \chi} \tilde{\phi}_{i}=\tilde{\phi}_{i}(\nabla \chi)$. The denominator of the right-hand side is easily computed thanks to Laplace's method:

$$
\sqrt{\int_{\Omega} \chi^{2} e^{-\frac{2}{h} f}}=\frac{(\pi h)^{\frac{d}{4}}}{\left(\operatorname{det} \operatorname{Hess} f\left(x_{0}\right)\right)^{1 / 4}} e^{-\frac{f\left(x_{0}\right)}{h}}(1+O(h)) .
$$

Using an integration by parts and the fact that $d^{*}\left(u_{h, i}^{(1)} e^{-f / h}\right)=0$ in $L^{2}\left(\dot{\Omega}_{i}\right)$ (see Proposition 83 items $(i i)$ and $(i i i)$ ) which is valid for all $h$ small enough, one obtains

$$
\begin{aligned}
\int_{\Omega} \nabla \chi \cdot \tilde{\phi}_{i} e^{-\frac{f}{h}} & =-\int_{\Omega} \nabla(1-\chi) \cdot \chi_{i} \frac{u_{h, i}^{(1)}}{\Theta_{i}} e^{-\frac{f}{h}} \\
& =\int_{\Omega}(1-\chi) \nabla \chi_{i} \cdot \frac{u_{h, i}^{(1)}}{\Theta_{i}} e^{-\frac{f}{h}}-\int_{\partial \Omega}(1-\chi) \tilde{\phi}_{i} \cdot n e^{-\frac{f}{h}} .
\end{aligned}
$$

Using the fact that $\chi=0$ on $\partial \Omega$, one then obtains:

$$
\begin{aligned}
\int_{\partial \Omega}(1-\chi) \tilde{\phi}_{i} \cdot n e^{-\frac{f}{h}} & =\int_{\partial \Omega \cap \operatorname{supp} \chi_{i}} \tilde{\phi}_{i} \cdot n e^{-\frac{f}{h}} \\
& =\int_{\Sigma_{i}} \tilde{\phi}_{i} \cdot n e^{-\frac{f}{h}}+\int_{\left(\partial \Omega \cap \operatorname{supp} \chi_{i}\right) \backslash \Sigma_{i}} \tilde{\phi}_{i} \cdot n e^{-\frac{f}{h}} .
\end{aligned}
$$

Using (240), in the limit $h \rightarrow 0$ :

$$
\begin{aligned}
\int_{\Omega} \nabla \chi \cdot \tilde{\phi}_{i} e^{-\frac{f}{h}}= & -B_{i} h^{m} e^{-\frac{1}{h} f\left(z_{i}\right)}(1+O(h)) \\
& -\int_{\left(\partial \Omega \cap \operatorname{supp} \chi_{i}\right) \backslash \Sigma_{i}} \tilde{\tilde{\phi}}_{i} \cdot n e^{-\frac{f}{h}}+\int_{\Omega}(1-\chi) \nabla \chi_{i} \cdot \frac{u_{h, i}^{(1)}}{\Theta_{i}} e^{-\frac{f}{h}} .
\end{aligned}
$$

Let us now prove that the two last terms in 242 are negligible compared to the first one. 
On the compact set $\left(\partial \Omega \cap \operatorname{supp} \chi_{i}\right) \backslash \Sigma_{i}$ one has $f(z)>f\left(z_{i}\right)$ since $z_{i} \in \Sigma_{i}$ is the only global minimum of $f$ on $B_{z_{i}}$ and supp $\chi_{i} \cap \partial \Omega \subset \Gamma_{1, i} \subset B_{z_{i}}$. Then, using Proposition 87 and (121), there exist $\varepsilon>0, h_{0}>0, C>0$ and $N \in \mathbb{N}$ such that for all $h \in\left(0, h_{0}\right)$

$$
\begin{aligned}
\mid \int_{\left(\partial \Omega \cap \operatorname{\phi } u p p \chi_{i}\right) \backslash \Sigma_{i}} \tilde{\tilde{\phi}}_{i} \cdot n e^{-\frac{f}{h}} & \leq e^{-\frac{f\left(z_{i}\right)+\varepsilon}{h}} \mid \int_{\left(\partial \Omega \cap \operatorname{supp} \chi_{i}\right) \backslash \Sigma_{i}} \tilde{\tilde{\phi}}_{i} \cdot n \\
& \leq C e^{-\frac{f\left(z_{i}\right)+\varepsilon}{h}}\left|\int_{\left(\partial \Omega \cap \operatorname{supp} \chi_{i}\right) \backslash \Sigma_{i}} \tilde{\phi}_{i} \cdot n e^{\frac{d_{a}\left(., z_{i}\right)}{h}}\right| \\
& \leq C \frac{e^{-\frac{f\left(z_{i}\right)+\varepsilon}{h}}}{\Theta_{i}}\left\|\chi_{i} u_{h, i}^{(1)} e^{\frac{d_{a}\left(., z_{i}\right)}{h}}\right\|_{H^{1}\left(\dot{\Omega}_{i}\right)} \\
& \leq C e^{-\frac{f\left(z_{i}\right)+\varepsilon}{h}} h^{-N} \leq C e^{-\frac{f\left(z_{i}\right)+\varepsilon / 2}{h}} .
\end{aligned}
$$

Let us now deal with the last term of 242 . The support of $(1-\chi) \nabla \chi_{i}$ is included in the support of $\nabla \chi_{i}$ and does not contain $x_{0}$ since $\chi \equiv 1$ around $x_{0}$. The function $\chi_{i}$ can be chosen such that the set $\left\{x \in \dot{\Omega}_{i},\left|\nabla \chi_{i}\right| \neq 0\right.$ and $\left.\chi \neq 1\right\}$ is as close as one wants from $\Gamma_{2, i}$ (see Figure 15). Therefore, by continuity of the Agmon distance, using (144), for any $\delta>0$, one can choose $\chi_{i}$ satisfying the three conditions stated in Definition 86 and such that

$$
\inf _{\operatorname{supp}(1-\chi) \nabla \chi_{i}}\left(d_{a}\left(\cdot, z_{i}\right)+f\right) \geq \inf _{B_{z_{i}}^{c}}\left(d_{a}\left(\cdot, z_{i}\right)+f\right)-\delta .
$$

Thus, using the Cauchy-Schwarz inequality and Proposition 87, there exists $N \in \mathbb{N}$ such that

$$
\begin{aligned}
\int_{\operatorname{supp}(1-\chi) \nabla \chi_{i}}\left|(1-\chi) \nabla \chi_{i} \cdot u_{h, i}^{(1)} e^{-\frac{f}{h}}\right| & \leq C\left\|u_{h, i}^{(1)} e^{\frac{d_{a}\left(\cdot, z_{i}\right)}{h}}\right\|_{L^{2}\left(\dot{\Omega}_{i}\right)} e^{-\frac{1}{h} \inf _{\operatorname{supp}(1-\chi) \nabla \chi_{i}}\left(d_{a}\left(\cdot, z_{i}\right)+f\right)} \\
& \leq C h^{-N} e^{-\frac{1}{h} \inf _{B_{z_{i}}^{c}}\left(d_{a}\left(\cdot, z_{i}\right)+f-\delta\right)} .
\end{aligned}
$$

Besides, from assumption 20 )

$$
\inf _{z \in B_{z_{i}}^{c}}\left[d_{a}\left(z, z_{i}\right)+f(z)\right]>f\left(z_{i}\right) .
$$

Indeed, the inequality 20 implies that there exists $r>0$ such that for all $z \in B_{z_{i}}^{c}$, $d_{a}\left(z, z_{i}\right) \geq f\left(z_{i}\right)-f\left(z_{1}\right)+r$ and therefore for all $z \in B_{z_{i}}^{c}$ one obtains

$$
d_{a}\left(z, z_{i}\right)+f(z) \geq f\left(z_{i}\right)+\left(f(z)-f\left(z_{1}\right)\right)+r \geq f\left(z_{i}\right)+r .
$$

Therefore, taking $\chi_{i}$ such that $\delta<r / 2$, one has, when $h \rightarrow 0$

$$
\int_{\operatorname{supp}(1-\chi) \nabla \chi_{i}}\left|(1-\chi) \nabla \chi_{i} \cdot u_{h, i}^{(1)} e^{-\frac{f}{h}}\right|=O\left(e^{-\frac{f\left(z_{i}\right)+c}{h}}\right)
$$

for some constant $c>0$.

In conclusion, for all $i \in\{1, \ldots, n\}$, one has in the limit $h \rightarrow 0$,

$$
\int_{\Omega} \nabla \tilde{u} \cdot \tilde{\phi}_{i} e^{-\frac{1}{h} f} d x=C_{i} h^{p} e^{-\frac{1}{h}\left(f\left(z_{i}\right)-f\left(x_{0}\right)\right)}(1+O(h)),
$$

with

$$
\left\{\begin{array}{l}
C_{i}=-B_{i} \frac{\left(\operatorname{det} \operatorname{Hess} f\left(x_{0}\right)\right)^{1 / 4}}{\pi^{\frac{d}{4}}}=-\frac{\pi^{-\frac{1}{4}} \sqrt{2 \partial_{n} f\left(z_{i}\right)}\left(\operatorname{det} \operatorname{Hess} f\left(x_{0}\right)\right)^{1 / 4}}{\left(\left.\operatorname{det} \operatorname{Hess} f\right|_{\partial \Omega}\left(z_{i}\right)\right)^{1 / 4}} \\
p=m-\frac{d}{4}=-\frac{3}{4}
\end{array}\right.
$$


where $B_{i}$ and $m$ have both been defined in (241). This concludes the proof of (58), and thus the proof of Theorem 1 .

\section{Consequences and generalizations of Theorem 1}

\subsection{Proofs of Proposition 12, Proposition 13, Corollary 14 and Corol- lary 16}

\subsubsection{Proof of Proposition 12}

Assume that [H1], [H2] and [H3] hold. From Lemma 26 and since the function $\tilde{u}$ is non negative in $\Omega$, there exists $h_{0}>0$ such that for all $h \in\left(0, h_{0}\right)$

$$
u_{h}=\frac{\pi_{h}^{(0)} \tilde{u}}{\left\|\pi_{h}^{(0)} \tilde{u}\right\|_{L_{w}^{2}}}
$$

where $u_{h}$ is the eigenfunction associated with the smallest eigenvalue of $-L_{f, h}^{(D),(0)}(\Omega)$ (see Proposition 22) and $\tilde{u}$ is introduced in Definition 84. Then, there exists $h_{0}>0$ such that for all $h \in\left(0, h_{0}\right)$,

$$
\int_{\Omega} u_{h} e^{-\frac{2}{h} f}=\int_{\Omega} \frac{\pi_{h}^{(0)} \tilde{u}}{\left\|\pi_{h}^{(0)} \tilde{u}\right\|_{L_{w}^{2}}} e^{-\frac{2}{h} f}=\frac{1}{\left\|\pi_{h}^{(0)} \tilde{u}\right\|_{L_{w}^{2}}} \int_{\Omega}\left[\tilde{u}+\left(\pi_{h}^{(0)}-1\right) \tilde{u}\right] e^{-\frac{2}{h} f}
$$

From the definition of $\chi$ (see Definition 84) and using Laplace's method, one obtains (in the limit $h \rightarrow 0$ )

$$
\int_{\Omega} \chi^{2} e^{-\frac{2}{h} f}=\frac{h^{\frac{d}{2}} \pi^{\frac{d}{2}}}{\sqrt{\operatorname{det} \operatorname{Hess} f\left(x_{0}\right)}} e^{-\frac{2}{h} f\left(x_{0}\right)}(1+O(h))
$$

and likewise

$$
\int_{\Omega} \chi e^{-\frac{2}{h} f}=\frac{h^{\frac{d}{2}} \pi^{\frac{d}{2}}}{\sqrt{\operatorname{det} \operatorname{Hess} f\left(x_{0}\right)}} e^{-\frac{2}{h} f\left(x_{0}\right)}(1+O(h)) .
$$

In addition, using Lemma 26 , one has $\left\|\pi_{h}^{(0)} \tilde{u}\right\|_{L_{w}^{2}}=1+O\left(e^{-\frac{c}{h}}\right)$. Therefore, it holds when $h \rightarrow 0$,

$$
\begin{aligned}
\frac{1}{\left\|\pi_{h}^{(0)} \tilde{u}\right\|_{L_{w}^{2}}} \int_{\Omega} \tilde{u} e^{-\frac{2}{h} f} & =\frac{\int_{\Omega} \chi e^{-\frac{2}{h} f}}{\sqrt{\int_{\Omega} \chi^{2} e^{-\frac{2 f}{h}}}}\left(1+O\left(e^{-\frac{c}{h}}\right)\right) \\
& =\frac{h^{\frac{d}{4}} \pi^{\frac{d}{4}}}{\left(\operatorname{det} \operatorname{Hess} f\left(x_{0}\right)\right)^{1 / 4}} e^{-\frac{1}{h} f\left(x_{0}\right)}(1+O(h)) .
\end{aligned}
$$

Moreover, from Lemma 26, there exist $c>0, h_{0}>0$ and $C>0$ such that for $h \in\left(0, h_{0}\right)$

$$
\frac{1}{\left\|\pi_{h}^{(0)} \tilde{u}\right\|_{L_{w}^{2}}}\left|\int_{\Omega}\left(\pi_{h}^{(0)}-1\right) \tilde{u} e^{-\frac{2}{h} f}\right| \leq C\left\|\left(1-\pi_{h}^{(0)}\right) \tilde{u}\right\|_{L_{w}^{2}} \sqrt{\int_{\Omega} e^{-\frac{2}{h} f}} \leq C e^{-\frac{c}{h}} e^{-\frac{1}{h} f\left(x_{0}\right)}
$$

Thus, one has when $h \rightarrow 0$,

$$
\int_{\Omega} u_{h} e^{-\frac{2}{h} f}=\frac{\pi^{\frac{d}{4}}}{\left(\operatorname{det} \operatorname{Hess} f\left(x_{0}\right)\right)^{1 / 4}} h^{\frac{d}{4}} e^{-\frac{1}{h} f\left(x_{0}\right)}(1+O(h)) .
$$

This proves Proposition 12 . 


\subsubsection{Proof of Proposition 13}

The aim of this section is to prove (23). To this end, we first state in Proposition 93 some estimates that the quasi-modes constructed in Section 4.2 satisfy under hypotheses [H1], [H2] and [H3]. Let us emphasize that these estimates are weaker than those obtained in Section 4.5 where in addition to [H1]-[H2]-[H3], the hypotheses $(20)$ and $(21)$ were also assumed. Then, we prove that the estimates of Proposition 93 imply $(23)$.

Proposition 93. Let us assume that the hypotheses [H1], [H2] and [H3] hold. Then there exist $n+1$ quasi-modes $\left(\left(\tilde{\psi}_{i}\right)_{i=1, \ldots, n}, \tilde{u}\right)$ which satisfy the following estimates:

1. $\forall i \in\{1, \ldots, n\}, \tilde{\psi}_{i} \in \Lambda^{1} H_{w, T}^{1}(\Omega)$ and $\tilde{u} \in \Lambda^{0} H_{w, T}^{1}(\Omega)$. The function $\tilde{u}$ is non negative in $\Omega$. Moreover $\forall i \in\{1, \ldots, n\},\left\|\tilde{\psi}_{i}\right\|_{L_{w}^{2}}=\|\tilde{u}\|_{L_{w}^{2}}=1$.

2. (a) There exists $\varepsilon_{1}>0$, for all $i \in\{1, \ldots, n\}$, in the limit $h \rightarrow 0$ :

$$
\left\|\left(1-\pi_{h}^{(1)}\right) \tilde{\psi}_{i}\right\|_{H_{w}^{1}}^{2}=O\left(e^{-\frac{\varepsilon_{1}}{h}}\right) .
$$

(b) For any $\delta>0,\|\nabla \tilde{u}\|_{L_{w}^{2}}^{2}=O\left(e^{-\frac{2}{h}\left(f\left(z_{1}\right)-f\left(x_{0}\right)-\delta\right)}\right)$.

3. There exists $\varepsilon_{0}>0$ such that $\forall(i, j) \in\{1, \ldots, n\}^{2}, i<j$, in the limit $h \rightarrow 0$ : $\left\langle\tilde{\psi}_{i}, \tilde{\psi}_{j}\right\rangle_{L_{w}^{2}}=O\left(e^{-\frac{\varepsilon_{0}}{h}}\right)$.

4. There exist $\varepsilon_{0}>0$, such that for all $i \in\{1, \ldots, n\}$, in the limit $h \rightarrow 0$ :

$$
\left\langle\nabla \tilde{u}, \tilde{\psi}_{i}\right\rangle_{L_{w}^{2}}=C_{i} h^{p} e^{-\frac{1}{h}\left(f\left(z_{i}\right)-f\left(x_{0}\right)\right)}(1+O(h))+O\left(e^{-\frac{1}{h}\left(f\left(z_{1}\right)-f\left(x_{0}\right)+\varepsilon_{0}\right)}\right),
$$

where the constants $p$ and $\left(C_{i}\right)_{i=1, \ldots, n}$ are given by 245).

Proof. Thanks to the hypotheses [H1], [H2] and [H3], one can introduce the $n+1$ quasimodes $\left(\left(\tilde{\phi}_{i}\right)_{i=1, \ldots, n}, \tilde{u}\right)$ built in Section 4.2 . Recall that $\tilde{\psi}_{i}=e^{\frac{1}{h} f} \tilde{\phi}_{i}$ for $i \in\{1, \ldots, n\}$.

Then, one easily obtains that $\left(\left(\tilde{\psi}_{i}\right)_{i=1, \ldots, n}, \tilde{u}\right)$ satisfy the estimates stated in Proposition 93. following exactly the computations made on $\left(\left(\tilde{\phi}_{i}\right)_{i=1, \ldots, n}, \tilde{u}\right)$ in Section 4.5: 2(a) follows from 234, 2(b) is a consequence of Lemma 85,3 follows from 238) and 4 is a consequence of (242)-(243)-(244) (in (244), one uses that for $\delta>0$ small enough, there exists $c>0$ such that $\left.\inf _{B_{z_{i}^{c}}}\left(d_{a}\left(., z_{i}\right)+f-\delta\right) \geq f\left(z_{1}\right)+c\right)$.

Let us now prove that the estimates stated in Proposition 93 imply (23), which will conclude the proof of Proposition 13.

Proof. From 12 together with the assumption $\left\|u_{h}\right\|_{L_{w}^{2}}=1$, it holds

$$
\lambda_{h}=-\left\langle L_{f, h}^{D,(0)}(\Omega) u_{h}, u_{h}\right\rangle_{L_{w}^{2}}=\frac{h}{2}\left\|\nabla u_{h}\right\|_{L_{w}^{2}}^{2}
$$

where $u_{h}$ is the eigenfunction associated with the smallest eigenvalue of $-L_{f, h}^{D,(0)}(\Omega)$ (see Proposition 22. Recall that $\nabla u_{h} \in \operatorname{Ran} \pi_{h}^{(1)}$ (see (52)).

In addition, let us recall that from items 1, 2(a) and 3 in Proposition 93 and using the proof of Lemma 27, there exists $h_{0}$ such that for all $h \in\left(0, h_{0}\right)$,

$$
\operatorname{span}\left(\pi_{h}^{(1)} \tilde{\psi}_{i}, i=1, \ldots, n\right)=\operatorname{Ran} \pi_{h}^{(1)} .
$$


Let us denote by $\left(\psi_{i}\right)_{i=1, \ldots, n}$ the orthornormal basis of Ran $\pi_{h}^{(1)}$ resulting from the GramSchmidt orthonormalisation procedure applied to the set $\left(\pi_{h}^{(1)} \tilde{\psi}_{i}\right)_{i=1, \ldots, n}$ (see Lemma 28 . so that

$$
\left\|\nabla u_{h}\right\|_{L_{w}^{2}}^{2}=\sum_{j=1}^{n}\left|\left\langle\nabla u_{h}, \psi_{j}\right\rangle_{L_{w}^{2}}\right|^{2} .
$$

We now want to estimate the terms $\left\langle\nabla u_{h}, \psi_{j}\right\rangle_{L_{w}^{2}}$.

Using 2(b) in Proposition 93 and using the proof of Lemma 26, one has that for $h$ small enough $\pi_{h}^{(0)} \tilde{u} \neq 0$ and therefore, since moreover $\tilde{u}$ is non negative in $\Omega, u_{h}=$ $\frac{\pi_{h}^{(0)} \tilde{u}}{\left\|\pi_{h}^{(0)} \tilde{u}\right\|_{L_{w}^{2}}}$. Thus one has (see 67 ), for $j \in\{1, \ldots, n\}$,

$$
\begin{aligned}
\left\langle\nabla u_{h}, \psi_{j}\right\rangle_{L_{w}^{2}}= & \frac{Z_{j}^{-1}}{\left\|\pi_{h}^{(0)} \tilde{u}\right\|_{L_{w}^{2}}}\left[\left\langle\nabla \tilde{u}, \tilde{\psi}_{j}\right\rangle_{L_{w}^{2}}+\left\langle\nabla \tilde{u},\left(\pi_{h}^{(1)}-1\right) \tilde{\psi}_{j}\right\rangle_{L_{w}^{2}}\right] \\
& +\frac{Z_{j}^{-1}}{\left\|\pi_{h}^{(0)} \tilde{u}\right\|_{L_{w}^{2}}}\left[\sum_{i=1}^{j-1} \kappa_{j i}\left(\left\langle\nabla \tilde{u}, \tilde{\psi}_{i}\right\rangle_{L_{w}^{2}}+\left\langle\nabla \tilde{u},\left(\pi_{h}^{(1)}-1\right) \tilde{\psi}_{i}\right\rangle_{L_{w}^{2}}\right)\right]
\end{aligned}
$$

where $\left(\kappa_{j i}\right)_{1 \leq i<j \leq n}$ and $\left(Z_{j}\right)_{1 \leq j \leq n}$ are defined in Lemma 28

Now, using the items 1, 2(a) and 3 of Proposition 93 and the proof of Lemma 30, one obtains that there exist $\varepsilon_{0}>0$ and $h_{0}>0$ such that $\forall h \in\left(0, h_{0}\right), \forall(i, j) \in\{1, \ldots, n\}^{2}$, it holds

$$
\kappa_{j i}=O\left(e^{-\frac{\varepsilon_{0}}{h}}\right) \text { and } Z_{i}=1+O\left(e^{-\frac{\varepsilon_{0}}{h}}\right),
$$

Injecting (248) and the estimates 2 and 4 of Proposition 93 into (67) leads to the existence of $\varepsilon^{\prime}>0$ and $h_{0}>0$ such that $\forall h \in\left(0, h_{0}\right)$,

$$
\left\langle\nabla u_{h}, \psi_{j}\right\rangle_{L_{w}^{2}}=C_{i} h^{p} e^{-\frac{1}{h}\left(f\left(z_{j}\right)-f\left(x_{0}\right)\right)}(1+O(h))+O\left(e^{-\frac{1}{h}\left(f\left(z_{1}\right)-f\left(x_{0}\right)+\varepsilon^{\prime}\right)}\right),
$$

where the constants $p$ and $\left(C_{i}\right)_{i=1, \ldots, n}$ are given by 245). Using 249) in 246) and 247), there exists $\varepsilon^{\prime}>0$ such that in the limit $h \rightarrow 0$

$$
\lambda_{h}=\frac{h}{2} \sum_{j=1}^{n} C_{i}^{2} h^{2 p} e^{-\frac{2}{h}\left(f\left(z_{i}\right)-f\left(x_{0}\right)\right)}(1+O(h))+O\left(e^{-\frac{2}{h}\left(f\left(z_{1}\right)-f\left(x_{0}\right)+\varepsilon^{\prime}\right)}\right) .
$$

Therefore, the estimate $(23)$ holds and Proposition 13 is proved.

\subsubsection{Proof of Corollary 14}

According to (14) one has for $i \in\{1, \ldots, n\}$ :

$$
\mathbb{P}_{\nu_{h}}\left[X_{\tau_{\Omega}} \in \Sigma_{i}\right]=-\frac{h}{2 \lambda_{h}} \frac{\int_{\Sigma_{i}}\left(\partial_{n} u_{h}\right)(z) e^{-\frac{2}{h} f(z)} \sigma(d z)}{\int_{\Omega} u_{h}(y) e^{-\frac{2}{h} f(y)} d y} .
$$

Let us assume that [H1], [H2] and [H3] together with the inequalities (20) and (21) hold. From Propositions 12 and 13, one obtains when $h \rightarrow 0$

$$
\begin{aligned}
\lambda_{h} \frac{2}{h} \int_{\Omega} u_{h} e^{-\frac{2}{h} f} d x= & 2 \pi^{\frac{d-2}{4}}\left(\operatorname{det} \operatorname{Hess} f\left(x_{0}\right)\right)^{\frac{1}{4}} h^{\frac{d-6}{4}} \\
& \times \sum_{k=1}^{n_{0}} \frac{\partial_{n} f\left(z_{k}\right)}{\sqrt{\left.\operatorname{det} \operatorname{Hess} f\right|_{\partial \Omega}\left(z_{k}\right)}} e^{-\frac{1}{h}\left(2 f\left(z_{1}\right)-f\left(x_{0}\right)\right)}(1+O(h)) .
\end{aligned}
$$


Then, using in addition Theorem 1 to estimate $\int_{\Sigma_{i}}\left(\partial_{n} u_{h}\right) e^{-\frac{2}{h} f} d \sigma(i \in\{1, \ldots, n\})$, one proves Corollary 14.

\subsubsection{Proof of Corollary 16}

Before starting the proof of Corollary 16 , let us notice that under the assumptions stated in Corollary 14, for all $i \in\{1, \ldots, n\}$ and for any test function $F \in C^{\infty}(\partial \Omega)$ satisfying $\operatorname{supp} F \subset B_{z_{i}}$ and $z_{i} \in \operatorname{int}(\operatorname{supp} F)$, when $h \rightarrow 0$,

$\mathbb{E}_{\nu_{h}}\left[F\left(X_{\tau_{\Omega}}\right)\right]=\frac{\partial_{n} f\left(z_{i}\right)}{\sqrt{\operatorname{det} \operatorname{Hess} f_{\mid \partial \Omega}\left(z_{i}\right)}}\left(\sum_{k=1}^{n_{0}} \frac{\partial_{n} f\left(z_{k}\right)}{\sqrt{\operatorname{det} \operatorname{Hess} f_{\mid \partial \Omega}\left(z_{k}\right)}}\right)^{-1} e^{-\frac{2}{h}\left(f\left(z_{i}\right)-f\left(z_{1}\right)\right)}\left(F\left(z_{i}\right)+O(h)\right)$.

The strategy for the proof of Corollary 16 is to first extend (250) to a deterministic initial condition, and then to deduce the result of Corollary 16 . To this end, let $i_{0} \in\{2, \ldots, n\}$ be as in $(26), j \in\left\{1, \ldots, i_{0}\right\}$ and $\alpha \in \mathbb{R}$ be such that

$$
f\left(x_{0}\right)<\alpha<2 f\left(z_{1}\right)-f\left(z_{j}\right) .
$$

Notice that we can assume without loss of generality (up to increasing $\alpha$ if $\alpha$ is smaller than $f\left(x_{0}\right)+f\left(z_{j}\right)-f\left(z_{1}\right)$, see (26)) that

$$
f\left(x_{0}\right)+f\left(z_{j}\right)-f\left(z_{1}\right)<\alpha<2 f\left(z_{1}\right)-f\left(z_{j}\right) .
$$

For such an $\alpha$, let us define

$$
K_{\alpha}:=f^{-1}((-\infty, \alpha]) \cap \Omega .
$$

For $i \in\{1, \ldots, j\}$, we would like to show that 250 holds when $X_{0}=x \in K_{\alpha}$, for any test function $F \in C^{\infty}(\partial \Omega)$ satisfying $\operatorname{supp} F \subset B_{z_{i}}$ and $z_{i} \in \operatorname{int}(\operatorname{supp} F)$.

Let us introduce the principal eigenfunction $\tilde{u}_{h}$ of $-L_{f, h}^{D,(0)}(\Omega)$ :

$$
\left\{\begin{aligned}
-L_{f, h}^{(0)} \tilde{u}_{h} & =\lambda_{h} \tilde{u}_{h} \text { on } \Omega \\
\tilde{u}_{h} & =0 \text { on } \partial \Omega
\end{aligned}\right.
$$

with $\tilde{u}_{h}>0$ on $\Omega$ and normalized such that

$$
\int_{\Omega} \tilde{u}_{h}^{2} d x=1
$$

Notice that $u_{h}$ solution to 12 only differs from $\tilde{u}_{h}$ by a multiplicative constant so that, from Proposition 5

$$
\nu_{h}(d x)=Z_{h}(\Omega)^{-1} \tilde{u}_{h}(x) e^{-\frac{2}{h} f(x)} d x,
$$

where, for any set $O \subset \Omega$,

$$
Z_{h}(O):=\int_{O} \tilde{u}_{h} e^{-\frac{2}{h} f}
$$

For $F \in C^{\infty}(\partial \Omega)$, let us define

$$
w_{h}(x)=\mathbb{E}_{x}\left[F\left(X_{\tau_{\Omega}}\right)\right] \text { for all } x \in \bar{\Omega} .
$$


The function $w_{h}$ is such that: $\forall h>0$ and $x \in \Omega$,

$$
\left|w_{h}(x)\right| \leq\|F\|_{L^{\infty}} .
$$

Moreover, a standard Feynman-Kac formula shows that $w_{h}$ satisfies

$$
\left\{\begin{aligned}
L_{f, h}^{(0)} w_{h} & =0 \text { on } \Omega \\
w_{h} & =F \text { on } \partial \Omega
\end{aligned}\right.
$$

where, we recall, the differential operator $L_{f, h}^{(0)}$ is defined by 10$)$. Our objective is to compare $w_{h}(x)$ with $\mathbb{E}_{\nu_{h}}\left[F\left(X_{\tau_{\Omega}}\right)\right]$.

By the Markov property, using 254, we have

$$
\begin{aligned}
\mathbb{E}_{\nu_{h}}\left[F\left(X_{\tau_{\Omega}}\right)\right] & =\left(\int_{\Omega} \tilde{u}_{h} e^{-\frac{2}{h} f}\right)^{-1}\left(\int_{\Omega} w_{h} \tilde{u}_{h} e^{-\frac{2}{h} f}\right) \\
& =Z_{h}^{-1}(\Omega)\left(\int_{\Omega \backslash K_{\alpha}} w_{h} \tilde{u}_{h} e^{-\frac{2}{h} f}\right)+Z_{h}^{-1}(\Omega)\left(\int_{K_{\alpha}} w_{h} \tilde{u}_{h} e^{-\frac{2}{h} f}\right) .
\end{aligned}
$$

In order to estimate the first term in (256), we need a leveling property for $\tilde{u}_{h}$, which is stated in 21, Theorem 2.4].

Lemma 94. Let us assume that [H1], [H2] and [H3] hold and let us consider $\tilde{u}_{h}$ the principal eigenfunction of $L_{f, h}^{D,(0)}(\Omega)$ (see 252) with normalization (253). Then, for any compact set $K \subset \Omega$,

$$
\lim _{h \rightarrow 0}\left\|\tilde{u}_{h}-\left(\int_{\Omega} d x\right)^{-1 / 2}\right\|_{L^{\infty}(K)}=0 .
$$

Notice that the reason why we consider a smooth test function $F$ rather than $1_{\Sigma_{i}}$ is that we would like to apply the results in 21.

A direct consequence of Lemma 94 is the following limit,

$$
\lim _{h \rightarrow 0} h \ln \left(Z_{h}(\Omega)\right)=-2 f\left(x_{0}\right) .
$$

Indeed, from the normalization of $\tilde{u}_{h}$, we get $Z_{h}(\Omega) \leq e^{-\frac{2}{h} f\left(x_{0}\right)}$, and from Lemma 94 we have, for $h$ small enough and for $r>0$ such that the open ball $B\left(x_{0}, 2 r\right)$ is included in $\Omega$,

$$
Z_{h}(\Omega) \geq Z_{h}\left(B\left(x_{0}, r\right)\right) \geq \frac{1}{2}\left(\int_{\Omega} d x\right)^{-1 / 2} \int_{B\left(x_{0}, r\right)} e^{-\frac{2 f}{h}} .
$$

Since $\lim _{h \rightarrow 0} h \ln \left(\int_{B\left(x_{0}, r\right)} e^{-\frac{2 f}{h}} d x\right)=-2 f\left(x_{0}\right)$, we get (257).

Let us now consider $i \in\{1, \ldots, j\}$. For the first term in 256), using 257) and 253), we have for any $\delta>0$, for $h$ small enough,

$$
\begin{aligned}
Z_{h}(\Omega)^{-1}\left|\int_{\Omega \backslash K_{\alpha}} w_{h} \tilde{u}_{h} e^{-\frac{2}{h} f}\right| & \leq\|F\|_{L^{\infty}} e^{\frac{\delta}{h}} e^{-\frac{2}{h}\left(\inf _{\Omega \backslash K_{\alpha}} f-f\left(x_{0}\right)\right)} \\
& =\|F\|_{L^{\infty}} e^{\frac{\delta}{h}} e^{-\frac{2}{h}\left(\alpha-f\left(x_{0}\right)\right)}
\end{aligned}
$$


and thus, thanks to 251 and the fact that $f\left(z_{i}\right) \leq f\left(z_{j}\right)$, by choosing $\delta$ small enough, there exists $c>0$ such that, for all $h$ small enough,

$$
Z_{h}(\Omega)^{-1}\left|\int_{\Omega \backslash K_{\alpha}} w_{h} \tilde{u}_{h} e^{-\frac{2}{h} f}\right| \leq\|F\|_{L^{\infty}} e^{-\frac{2}{h}\left(f\left(z_{i}\right)-f\left(z_{1}\right)+c\right)} .
$$

In order to estimate the second term in 256), we need a leveling property for $w_{h}$.

Lemma 95. Let us assume that [H1], [H2] and [H3] hold, as well as (251). Let us consider $w_{h}$ solution to 255. Then there exists $C>0$ such that for any $\delta>0$, for any $h$ small enough, for all $x, y \in K_{\alpha}$,

$$
\left|w_{h}(x)-w_{h}(y)\right| \leq C e^{\frac{\delta}{h}} e^{-\frac{2}{h}\left(f\left(z_{1}\right)-\alpha\right)} .
$$

Proof. From [27, Theorem 1], it is known that for any $\delta>0$, for any $h$ small enough and for all $x, y \in K_{\alpha}$,

$$
\left|w_{h}(x)-w_{h}(y)\right| \leq C e^{\frac{\delta}{h}} e^{-\frac{2}{h} V_{\Omega}\left(K_{\alpha}\right)},
$$

where $V_{\Omega}\left(K_{\alpha}\right)$ is defined by,

$$
V_{\Omega}\left(K_{\alpha}\right)=\inf _{x \in K_{\alpha}} \inf _{T>0} \inf _{\gamma \in \operatorname{Abs}(T, x, \partial \Omega)} \frac{1}{4} \int_{0}^{T}|\dot{\gamma}+\nabla f(\gamma)|^{2} d t
$$

where $\operatorname{Abs}(T, x, \partial \Omega)$ is the set of absolutely continuous functions $\gamma:[0, T] \rightarrow \bar{\Omega}$ satisfying $\gamma(0)=x$ and $\gamma(T) \in \partial \Omega$. For any $\gamma \in \operatorname{Abs}(T, x, \partial \Omega)$, we have

$$
\int_{0}^{T}|\dot{\gamma}+\nabla f(\gamma)|^{2} d t-\int_{0}^{T}|\dot{\gamma}-\nabla f(\gamma)|^{2} d t=4 \int_{0}^{T} \dot{\gamma} \cdot \nabla f(\gamma) d t=4(f(\gamma(T))-f(x)),
$$

and therefore, it holds

$$
\int_{0}^{T}|\dot{\gamma}+\nabla f(\gamma)|^{2} d t \geq 4(f(\gamma(T))-f(x)) \geq 4\left(f\left(z_{1}\right)-f(x)\right) .
$$

Finally we obtain

$$
V_{\Omega}\left(K_{\alpha}\right) \geq f\left(z_{1}\right)-\max _{x \in K_{\alpha}} f(x)=f\left(z_{1}\right)-\alpha .
$$

This concludes the proof of Lemma 95 .

We are now in position to estimate the second term in 256. Using Lemma 95, we get, for any $\delta>0$, in the limit $h \rightarrow 0$, uniformly in $y_{0} \in K_{\alpha}$,

$$
Z_{h}^{-1}(\Omega)\left(\int_{K_{\alpha}} w_{h} \tilde{u}_{h} e^{-\frac{2}{h} f} d x\right)=w_{h}\left(y_{0}\right) \frac{Z_{h}\left(K_{\alpha}\right)}{Z_{h}(\Omega)}+O\left(e^{\frac{\delta}{h}} e^{-\frac{2}{h}\left(f\left(z_{1}\right)-\alpha\right)}\right) \frac{Z_{h}\left(K_{\alpha}\right)}{Z_{h}(\Omega)} .
$$

Therefore, by choosing $\delta>0$ small enough, thanks to 251 and the fact that $f\left(z_{i}\right) \leq$ $f\left(z_{j}\right)$ (since we recall that $i \in\{1, \ldots, j\}$ ), there exists $c>0$ such that, in the limit $h \rightarrow 0$,

$$
Z_{h}^{-1}(\Omega)\left(\int_{K_{\alpha}} w_{h} \tilde{u}_{h} e^{-\frac{2}{h} f} d x\right)=w_{h}\left(y_{0}\right) \frac{Z_{h}\left(K_{\alpha}\right)}{Z_{h}(\Omega)}+O\left(e^{-\frac{2}{h}\left(f\left(z_{i}\right)-f\left(z_{1}\right)+c\right)}\right) \frac{Z_{h}\left(K_{\alpha}\right)}{Z_{h}(\Omega)} .
$$


In addition we have $\frac{Z_{h}\left(K_{\alpha}\right)}{Z_{h}(\Omega)}=1+O\left(e^{-\frac{c}{h}}\right)$ for some $c>0$ independent of $h$. Indeed $\frac{Z_{h}\left(K_{\alpha}\right)}{Z_{h}(\Omega)}=1-\frac{Z_{h}\left(\Omega \backslash K_{\alpha}\right)}{Z_{h}(\Omega)}$ and using 257$)$, we get for any $\delta>0$,

$$
\frac{Z_{h}\left(\Omega \backslash K_{\alpha}\right)}{Z_{h}(\Omega)} \leq e^{\frac{\delta}{h}} e^{-\frac{2}{h}\left(\min _{\Omega \backslash K_{\alpha}} f-f\left(x_{0}\right)\right)}=O\left(e^{-\frac{c}{h}}\right),
$$

for some $c>0$ independent of $h$ by choosing $\delta$ small enough. Gathering the results 258)-259-260) in 256), for all $i \in\{1, \ldots, j\}$, there exists $c>0$ independent of $h$ such that, in the limit $h \rightarrow 0$, it holds: uniformly in $y_{0} \in K_{\alpha}$,

$$
\mathbb{E}_{\nu_{h}}\left[F\left(X_{\tau_{\Omega}}\right)\right]=w_{h}\left(y_{0}\right)\left(1+O\left(e^{-\frac{c}{h}}\right)\right)+O\left(e^{-\frac{2}{h}\left(f\left(z_{i}\right)-f\left(z_{1}\right)+c\right)}\right) .
$$

Let $i \in\{1, \ldots, j\}$ and let us assume that $\operatorname{supp} F \subset B_{z_{i}}$ and $z_{i} \in \operatorname{int}(\operatorname{supp} F)$. Then, combining the last estimate with 250) implies that uniformly in $x \in f^{-1}((-\infty, \alpha]) \cap \Omega$, in the limit $h \rightarrow 0$ :

$\mathbb{E}_{x}\left[F\left(X_{\tau_{\Omega}}\right)\right]=\frac{\partial_{n} f\left(z_{i}\right)}{\sqrt{\operatorname{det} \operatorname{Hess} f_{\mid \partial \Omega}\left(z_{i}\right)}}\left(\sum_{k=1}^{n_{0}} \frac{\partial_{n} f\left(z_{k}\right)}{\sqrt{\operatorname{det} \operatorname{Hess} f_{\mid \partial \Omega}\left(z_{k}\right)}}\right)^{-1} e^{-\frac{2}{h}\left(f\left(z_{i}\right)-f\left(z_{1}\right)\right)}\left(F\left(z_{i}\right)+O(h)\right)$.

Let $\Sigma_{i} \subset \partial \Omega$ containing $z_{i}$ and such that $\bar{\Sigma}_{i} \subset B_{z_{i}}$. Then, there exit $F, G \in C^{\infty}(\partial \Omega)$ such that $\operatorname{supp} F \subset B_{z_{i}}, z_{i} \in \operatorname{int}(\operatorname{supp} F), \operatorname{supp} G \subset B_{z_{i}}, z_{i} \in \operatorname{int}(\operatorname{supp} G), F \leq 1_{\Sigma_{i}} \leq G$ and $F\left(z_{i}\right)=G\left(z_{i}\right)=1$. From the inequality

$$
\mathbb{E}_{x}\left[F\left(X_{\tau_{\Omega}}\right)\right] \leq \mathbb{P}_{x}\left[X_{\tau_{\Omega}} \in \Sigma_{i}\right] \leq \mathbb{E}_{x}\left[G\left(X_{\tau_{\Omega}}\right)\right],
$$

together with 261) applied to $F$ and $G$, one gets, for all $i \in\{1, \ldots, j\}$, in the limit $h \rightarrow 0$ :

$\mathbb{P}_{x}\left[X_{\tau_{\Omega}} \in \Sigma_{i}\right]=\frac{\partial_{n} f\left(z_{i}\right)}{\sqrt{\operatorname{det} \operatorname{Hess} f_{\mid \partial \Omega}\left(z_{i}\right)}}\left(\sum_{k=1}^{n_{0}} \frac{\partial_{n} f\left(z_{k}\right)}{\sqrt{\operatorname{det} \operatorname{Hess} f_{\mid \partial \Omega}\left(z_{k}\right)}}\right)^{-1} e^{-\frac{2}{h}\left(f\left(z_{i}\right)-f\left(z_{1}\right)\right)}(1+O(h))$.

This concludes the proof of Corollary 16 .

\subsection{Proofs of Theorem 2 and Corollary 17}

In this section, we prove Theorem 2, The proof is similar to the one made for Theorem 1 the estimates of Proposition 25 and the construction of the quasi-mode associated with $z_{j_{0}}$ are modified. The proof of Theorem 2 is organized as follows. In Section 5.2.1. we give the estimates required for the $n+1$ quasi-modes. Then, in Section 5.2.2, we prove that these estimates imply Theorem 2. In Section 5.2.3, the construction of the quasi-modes is given and we check that they satisfy the estimates stated in Section 5.2.1.

\subsubsection{Statement of the assumptions required for the quasi-modes}

Let su recall that, for $p \in\{0,1\}$, the orthogonal projector $\pi_{\left[0, \frac{\sqrt{h}}{2}\right)}\left(-L_{f, h}^{D,(p)}(\Omega)\right)$ is still denoted by $\pi_{h}^{(p)}$, see $(53)$.

The next proposition gives the assumptions needed on the quasi-modes $\left(\tilde{\psi}_{i}\right)_{i=1, \ldots, n}$ whose span approximates $\operatorname{Ran} \pi_{h}^{(1)}$, and $\tilde{u}$ whose span approximates $\operatorname{Ran} \pi_{h}^{(0)}$, in order to prove Theorem 2. It is the equivalent of Proposition 25 in the more general setting of Theorem 2 , 
Proposition 96. Let us assume that the hypotheses [H1], [H2] and [H3] hold. Let $\Sigma_{i}$ denotes an open set included in $\partial \Omega$ containing $z_{i}(i \in\{1, \ldots, n\})$ and such that $\bar{\Sigma}_{i} \subset B_{z_{i}}$. Let $k_{0} \in\{1, \ldots, n\}$ and $f^{*}$ such that

$$
f\left(z_{k_{0}}\right) \leq f^{*} \leq f\left(z_{k_{0}+1}\right),
$$

(with the convention $f\left(z_{k_{0}+1}\right)=+\infty$ if $k_{0}=n$ ). Finally, let $\Sigma \subset \partial \Omega$ be a smooth open set such that $\inf _{\Sigma} f=f^{*}$ and $\bar{\Sigma} \subset B_{z_{j_{0}}}$, for some $j_{0} \in\left\{1, \ldots, k_{0}\right\}$.

Let us assume that there exist $n$ quasi-modes $\left(\tilde{\psi}_{i}\right)_{i=1, \ldots, n}$ and a family of quasi-modes $\left(\tilde{u}=\tilde{u}_{\delta}\right)_{\delta>0}$ satisfying the following conditions:

1. $\forall i \in\{1, \ldots, n\}, \tilde{\psi}_{i} \in \Lambda^{1} H_{w, T}^{1}(\Omega)$ and $\tilde{u} \in \Lambda^{0} H_{w, T}^{1}(\Omega)$. The function $\tilde{u}$ is non negative in $\Omega$. Moreover, $\forall i \in\{1, \ldots, n\},\left\|\tilde{\psi}_{i}\right\|_{L_{w}^{2}}=\|\tilde{u}\|_{L_{w}^{2}}=1$.

2. (a) There exists $\varepsilon_{1}>0$ such that for all $i \in\left\{1, \ldots, k_{0}\right\}$, it holds in the limit $h \rightarrow 0$ :

$$
\left\|\left(1-\pi_{h}^{(1)}\right) \tilde{\psi}_{i}\right\|_{H_{w}^{1}}^{2}=O\left(e^{-\frac{2}{h}\left(\max \left[f^{*}-f\left(z_{i}\right), f\left(z_{i}\right)-f\left(z_{1}\right)\right]+\varepsilon_{1}\right)}\right)
$$

and for all $i \in\left\{k_{0}+1, \ldots, n\right\},\left\|\left(1-\pi_{h}^{(1)}\right) \tilde{\psi}_{i}\right\|_{H_{w}^{1}}^{2}=O\left(e^{-\frac{2}{h}\left(f^{*}-f\left(z_{1}\right)+\varepsilon_{1}\right)}\right)$.

(b) For any $\delta>0$, in the limit $h \rightarrow 0:\|\nabla \tilde{u}\|_{L_{w}^{2}}^{2}=O\left(e^{-\frac{2}{h}\left(f\left(z_{1}\right)-f\left(x_{0}\right)-\delta\right)}\right)$.

3. There exists $\varepsilon_{0}>0, \forall(i, j) \in\{1, \ldots, n\}^{2}$, in the limit $h \rightarrow 0$, if $i<j \leq k_{0}$ :

$$
\left\langle\tilde{\psi}_{i}, \tilde{\psi}_{j}\right\rangle_{L_{w}^{2}}=O\left(e^{-\frac{1}{h}\left(f\left(z_{j}\right)-f\left(z_{i}\right)+\varepsilon_{0}\right)}\right),
$$

and, if $k_{0}<j, i<j$ :

$$
\left\langle\tilde{\psi}_{i}, \tilde{\psi}_{j}\right\rangle_{L_{w}^{2}}=O\left(e^{-\frac{1}{h}\left(f^{*}-f\left(z_{1}\right)+\varepsilon_{0}\right)}\right) .
$$

4. (a) There exists $\varepsilon^{\prime}>0$ and there exist constants $\left(C_{i}\right)_{i=1, \ldots, n}$ and $p$ which do not depend on $h$ such that, in the limit $h \rightarrow 0$, for $i \in\left\{1, \ldots, k_{0}\right\}$ :

$$
\left\langle\nabla \tilde{u}, \tilde{\psi}_{i}\right\rangle_{L_{w}^{2}}=C_{i} h^{p} e^{-\frac{1}{h}\left(f\left(z_{i}\right)-f\left(x_{0}\right)\right)}(1+O(h)),
$$

and for $i \in\left\{k_{0}+1, \ldots, n\right\}$ :

$$
\left\langle\nabla \tilde{u}, \tilde{\psi}_{i}\right\rangle_{L_{w}^{2}}=C_{i} h^{p} e^{-\frac{1}{h}\left(f\left(z_{i}\right)-f\left(x_{0}\right)\right)}(1+O(h))+O\left(e^{-\frac{1}{h}\left(f^{*}-f\left(x_{0}\right)+\varepsilon^{\prime}\right)}\right) .
$$

(b) There exist constants $\left(B_{i}\right)_{i=1, \ldots, n}$ and $m$ which do not depend on $h$ such that for all $(i, j) \in\{1, \ldots, n\}^{2}$, in the limit $h \rightarrow 0$ :

$$
\int_{\Sigma_{i}} \tilde{\psi}_{j} \cdot n e^{-\frac{2}{h} f} d \sigma= \begin{cases}B_{i} h^{m} e^{-\frac{1}{h} f\left(z_{i}\right)}(1+O(h)) & \text { if } i=j \\ 0 & \text { if } i \neq j .\end{cases}
$$

(c) There exist $C^{*}$ and $p^{*}$ independent of $h$ such that for all $i \in\{1, \ldots, n\}$, in the limit $h \rightarrow 0$ :

$$
\int_{\Sigma} \tilde{\psi}_{i} \cdot n e^{-\frac{2}{h} f} d \sigma=\delta_{j_{0}, i} C^{*} h^{q^{*}} e^{-\frac{1}{h}\left(2 f^{*}-f\left(z_{j_{0}}\right)\right)}(1+O(h)) .
$$


Let $u_{h}$ be the eigenfunction associated with the smallest eigenvalue of $-L_{f, h}^{D,(0)}(\Omega)$ (see Proposition 22) which satisfies (11). Then, one has:

- For all $i \in\left\{1, \ldots, k_{0}\right\}$, in the limit $h \rightarrow 0$

$$
\int_{\Sigma_{i}}\left(\partial_{n} u_{h}\right) e^{-\frac{2}{h} f} d \sigma=C_{i} B_{i} h^{p+m} e^{-\frac{1}{h}\left(2 f\left(z_{i}\right)-f\left(x_{0}\right)\right)}(1+O(h)) .
$$

Moreover, if $f\left(z_{k_{0}}\right)<f\left(z_{k_{0}+1}\right)$, there exists $\varepsilon>0$ such that for all $i \in\left\{k_{0}+\right.$ $1, \ldots, n\}$ in the limit $h \rightarrow 0$

$$
\int_{\Sigma_{i}}\left(\partial_{n} u_{h}\right) e^{-\frac{2}{h} f} d \sigma=\left(\int_{\Sigma_{k_{0}}}\left(\partial_{n} u_{h}\right) e^{-\frac{2}{h} f} d \sigma\right) O\left(e^{-\frac{\varepsilon}{h}}\right) .
$$

- In the limit $h \rightarrow 0$ :

$$
\int_{\Sigma}\left(\partial_{n} u_{h}\right) e^{-\frac{2}{h} f} d \sigma=C^{*} C_{j_{0}} h^{q^{*}+p} e^{-\frac{1}{h}\left(2 f^{*}-f\left(x_{0}\right)\right)}(1+O(h)) .
$$

The asymptotic estimates (31) and (34) in Theorem 2 are consequences of this proposition and of the construction of some appropriate quasi-modes $\left(\left(\tilde{\psi}_{i}\right)_{i=1, \ldots, n}, \tilde{u}\right)$, see Section 5.2.3, which will show that $\left(B_{i}\right)_{i=1, \ldots, n}, m,\left(C_{i}\right)_{i=1, \ldots, n}, p$ are given by (241)(245) and $C^{*}, q^{*}$ will be given in Lemma 103 below. Moreover, the other asymptotic estimates (32) and (35) in Theorem 2 are consequences of the asymptotic estimates (31) and (34) together with Proposition 12 , Proposition 13 and (14).

\subsubsection{Proof of Proposition 96}

The proof of Proposition 96 follows closely the same steps as the proof of Proposition 25. We only highlight the main differences. In all this section, we assume that the hypotheses [H1], [H2] and [H3] hold. Let $f^{*} \in \mathbb{R}, k_{0} \in\{1, \ldots, n\}, j_{0} \in\left\{1, \ldots, k_{0}\right\},\left(\Sigma_{i}\right)_{i \in\{1, \ldots, n\}}$ and $\Sigma$ be as stated in Proposition 96. In addition, let us assume the existence of $n+1$ quasi-modes $\left(\tilde{u},\left(\tilde{\psi}_{i}\right)_{i=1, \ldots, n}\right)$ satisfying all the conditions of Proposition 96 . In the following, $\varepsilon$ denotes a positive constant independent of $h$, smaller than $\min \left(\varepsilon_{1}, \varepsilon_{0}, \varepsilon^{\prime}\right)$, and whose precise value may vary (a finite number of times) from one occurrence to the other.

Let us recall a result relating $\tilde{u}$ with $u_{h}$ on the one hand, and $\operatorname{span}\left(\tilde{\psi}_{j}, j=1, \ldots, n\right)$ with $\operatorname{Ran} \pi_{h}^{(1)}$ on the other hand. The following lemma is a direct consequence of Lemma 26, Lemma 27 and the assumptions 1, 2 and 3 of Proposition 96.

Lemma 97. Let us assume that the assumptions of Proposition 96 hold. Then, there exist $c>0$ and $h_{0}>0$ such that for $h \in\left(0, h_{0}\right)$,

$$
\left\|\pi_{h}^{(0)} \tilde{u}\right\|_{L_{w}^{2}}=1+O\left(e^{-\frac{c}{h}}\right) .
$$

In addition, there exists $h_{0}>0$ such that for all $h \in\left(0, h_{0}\right)$,

$$
\operatorname{span}\left(\pi_{h}^{(1)} \tilde{\psi}_{i}, i=1, \ldots, n\right)=\operatorname{Ran} \pi_{h}^{(1)} .
$$


A direct consequence of Lemma 97 and the fact that $\tilde{u}$ is non negative in $\Omega$ is that it holds for $h$ small enough:

$$
u_{h}=\frac{\pi_{h}^{(0)} \tilde{u}}{\left\|\pi_{h}^{(0)} \tilde{u}\right\|_{L_{w}^{2}}} .
$$

Let us denote by $\left(\psi_{i}\right)_{i=1, \ldots, n}$ the orthonormal basis of Ran $\pi_{h}^{(1)}$ resulting from the GramSchmidt orthonormalization procedure on the set $\left(\pi_{h}^{(1)} \tilde{\psi}_{i}\right)_{i=1, \ldots, n}$ (see Lemma 28). Then, since $\nabla u_{h} \in \operatorname{Ran}\left(\pi_{h}^{(1)}\right)=\operatorname{span}\left(\psi_{j}, j=1, \ldots, n\right)$ (see 52 ) and $\left\langle\psi_{j}, \psi_{i}\right\rangle_{L_{w}^{2}}=\delta_{i, j}$, one has for any $\Gamma \subset \partial \Omega$

$$
\int_{\Gamma}\left(\partial_{n} u_{h}\right) e^{-\frac{2}{h} f} d \sigma=\sum_{j=1}^{n}\left\langle\nabla u_{h}, \psi_{j}\right\rangle_{L_{w}^{2}} \int_{\Gamma} \psi_{j} \cdot n e^{-\frac{2}{h} f} d \sigma
$$

Let $\left(\kappa_{j i}\right)_{(i, j) \in\{1, \ldots, n\}^{2}, i<j}$ and $\left(Z_{j}\right)_{j \in\{1, \ldots, n\}}$ be the matrix and vector obtained through the Gram-Schimdt othonormalization procedure, see Lemma 28.

The strategy to prove Proposition 96 consists in estimating precisely the following terms in the limit $h \rightarrow 0$ :

$$
\kappa_{j i}, Z_{j},\left\langle\nabla u_{h}, \psi_{j}\right\rangle_{L_{w}^{2}} \text { and }\left(\int_{\Gamma} \psi_{j} \cdot n e^{-\frac{2}{h} f} d \sigma\right)_{\Gamma \in\left\{\Sigma, \Sigma_{1}, \ldots, \Sigma_{n}\right\}}
$$

for $(i, j) \in\{1, \ldots, n\}^{2}, i<j$. Then, they will be used to obtain a precise estimate of the right-hand-side of 263 when $h \rightarrow 0$.

Step 1. Estimates on the terms $\left(\kappa_{j i}\right)_{(i, j) \in\{1, \ldots, n\}^{2}, i<j}$ and $\left(Z_{i}\right)_{i \in\{1, \ldots, n\}}$.

Lemma 98. Let us assume that the assumptions of Proposition 96 hold. Then, there exist $\varepsilon>0$ and $h_{0}>0$ such that for all $(i, j) \in\{1, \ldots, n\}^{2}$ with $i<j$ and all $h \in\left(0, h_{0}\right)$, if $j \leq k_{0}$ :

$$
\left\langle\pi_{h}^{(1)} \tilde{\psi}_{i}, \pi_{h}^{(1)} \tilde{\psi}_{j}\right\rangle_{L_{w}^{2}}=O\left(e^{-\frac{1}{h}\left(f\left(z_{j}\right)-f\left(z_{i}\right)+\varepsilon\right)}\right),
$$

and if $j>k_{0}$ :

$$
\left\langle\pi_{h}^{(1)} \tilde{\psi}_{i}, \pi_{h}^{(1)} \tilde{\psi}_{j}\right\rangle_{L_{w}^{2}}=O\left(e^{-\frac{1}{h}\left(f^{*}-f\left(z_{1}\right)+\varepsilon\right)}\right) .
$$

Proof. The proof follows the same lines of the proof of Lemma 29. If $i<j$ and $j \leq k_{0}$, from assumption 2(a) in Proposition 96 and since $f^{*} \geq f\left(z_{1}\right)$, one gets

$$
\begin{aligned}
\left\langle\left(1-\pi_{h}^{(1)}\right) \tilde{\psi}_{i},\left(1-\pi_{h}^{(1)}\right) \tilde{\psi}_{j}\right\rangle_{L_{w}^{2}} & \leq\left\|\left(1-\pi_{h}^{(1)}\right) \tilde{\psi}_{i}\right\|_{L_{w}^{2}}\left\|\left(1-\pi_{h}^{(1)}\right) \tilde{\psi}_{j}\right\|_{L_{w}^{2}} \\
& \leq O\left(e^{-\frac{1}{h}\left(f^{*}-f\left(z_{i}\right)+f\left(z_{j}\right)-f\left(z_{1}\right)+\varepsilon\right)}\right)=O\left(e^{-\frac{1}{h}\left(f\left(z_{j}\right)-f\left(z_{i}\right)+\varepsilon\right)}\right) .
\end{aligned}
$$

If $i<j$ and $k_{0}<j$, from assumptions 1 and 2(a) in Proposition 96, one gets

$$
\left\langle\left(1-\pi_{h}^{(1)}\right) \tilde{\psi}_{i},\left(1-\pi_{h}^{(1)}\right) \pi_{h}^{(1)} \tilde{\psi}_{j}\right\rangle_{L_{w}^{2}} \leq\left\|\tilde{\psi}_{i}\right\|_{L_{w}^{2}}\left\|\left(1-\pi_{h}^{(1)}\right) \tilde{\psi}_{j}\right\|_{L_{w}^{2}} \leq O\left(e^{-\frac{1}{h}\left(f^{*}-f\left(z_{1}\right)+\varepsilon\right)}\right) .
$$

Lemma 98 is then a consequence of 61 together with assumption 3 in Proposition 96. 
Lemma 99. Let us assume that the assumptions of Proposition 96 hold. Then, there exist $\varepsilon>0$ and $h_{0}>0$ such that for all $(i, j) \in\{1, \ldots, n\}^{2}$ with $i<j$ and all $h \in\left(0, h_{0}\right)$, if $j \leq k_{0}$ :

$$
\kappa_{j i}=O\left(e^{-\frac{1}{h}\left(f\left(z_{j}\right)-f\left(z_{i}\right)+\varepsilon\right)}\right)
$$

and if $j>k_{0}$ :

$$
\kappa_{j i}=O\left(e^{-\frac{1}{h}\left(f^{*}-f\left(z_{1}\right)+\varepsilon\right)}\right) .
$$

In addition, there exist $c>0$ and $h_{0}>0$ such that for all $j \in\{1, \ldots, n\}$ and $h \in\left(0, h_{0}\right)$,

$$
Z_{j}=1+O\left(e^{-\frac{c}{h}}\right) .
$$

Proof. If $i<j$ and $j \leq k_{0}$, the estimates on $\kappa_{j i}$ and $Z_{j}$ are proved by induction as in the proof of Lemma 30. Let us now deal with the case $i<j$ and $k_{0}<j$. For $j=k_{0}+1$, it follows from (65) that for all $i<k_{0}+1$,

$$
\kappa_{\left(k_{0}+1\right) i}=-\sum_{k=i}^{k_{0}} \sum_{l=1}^{k} \frac{1}{Z_{k}^{2}}\left\langle\pi_{h}^{(1)} \tilde{\psi}_{k_{0}+1}, \pi_{h}^{(1)} \tilde{\psi}_{l}\right\rangle_{L_{w}^{2}} \kappa_{k l} \kappa_{k i},
$$

where we use the notation $\kappa_{i i}=1$ for every $i \in\{1, \ldots, n\}$. Since $1 \leq k \leq k_{0}$, one has $Z_{k}^{-1}=1+O\left(e^{-\frac{c}{h}}\right)$. In addition, since $1 \leq l \leq k \leq k_{0}$ and $1 \leq i \leq k \leq k_{0}$, one has $\kappa_{k l} \kappa_{k i}=O(1)$. From Lemma 98 , one has for $1 \leq l<k_{0}+1,\left\langle\pi_{h}^{(1)} \tilde{\psi}_{k_{0}+1}, \pi_{h}^{(1)} \tilde{\psi}_{l}\right\rangle_{L_{w}^{2}}=$ $O\left(e^{-\frac{1}{h}\left(f^{*}-f\left(z_{1}\right)+\varepsilon\right)}\right)$. Therefore, one obtains for all $i<k_{0}+1$,

$$
\kappa_{\left(k_{0}+1\right) i}=O\left(e^{-\frac{1}{h}\left(f^{*}-f\left(z_{1}\right)+\varepsilon\right)}\right) .
$$

The fact that $Z_{k_{0}+1}=1+O\left(e^{-\frac{c}{h}}\right)$, comes from the fact that the terms $\left(\kappa_{\left(k_{0}+1\right) i}\right)_{i \in\left\{1, \ldots, k_{0}\right\}}$ are exponentially small and the fact that $\left\|\pi_{h}^{(1)} \tilde{\psi}_{k_{0}+1}\right\|_{L_{w}^{2}}=1+O\left(e^{-\frac{c}{h}}\right)$. In order to prove by induction the estimates on $\kappa_{j i}$ for $i<j$ and $j>k_{0}$, let us now assume that for some $k \in\left\{k_{0}+1, \ldots, n\right\}$ and for all $j \in\left\{k_{0}+1, \ldots, k\right\}, i \in\{1, \ldots, j-1\}$,

$$
\kappa_{j i}=O\left(e^{-\frac{1}{h}\left(f^{*}-f\left(z_{1}\right)+\varepsilon\right)}\right) \text { and } Z_{j}=1+O\left(e^{-\frac{c}{h}}\right) .
$$

It follows from (65), for $q \in\{1, \ldots, k\}$,

$$
\kappa_{(k+1) q}=-\sum_{j=q}^{k} \sum_{l=1}^{j} \frac{1}{Z_{j}^{2}}\left\langle\pi_{h}^{(1)} \tilde{\psi}_{k+1}, \pi_{h}^{(1)} \tilde{\psi}_{l}\right\rangle_{L_{w}^{2}} \kappa_{j l} \kappa_{j q},
$$

where we used the notation $\kappa_{i i}=1$. Since $1 \leq j \leq k$, one has $Z_{j}^{-1}=1+O\left(e^{-\frac{c}{h}}\right)$. In addition, since $1 \leq l \leq j \leq k$ and $1 \leq q \leq j \leq k$, one has $\kappa_{j l} \kappa_{j q}=O(1)$. From Lemma 98 , one has for $1 \leq l<k+1$ and $k>k_{0},\left\langle\pi_{h}^{(1)} \tilde{\psi}_{k+1}, \pi_{h}^{(1)} \tilde{\psi}_{l}\right\rangle_{L_{w}^{2}}=O\left(e^{-\frac{1}{h}\left(f^{*}-f\left(z_{1}\right)+\varepsilon\right)}\right)$. Therefore, one obtains for all $1 \leq q<k+1$,

$$
\kappa_{(k+1) q}=O\left(e^{-\frac{1}{h}\left(f^{*}-f\left(z_{1}\right)+\varepsilon\right)}\right) .
$$

The fact that $Z_{k+1}=1+O\left(e^{-\frac{c}{h}}\right)$, comes from the fact that the $\left(\kappa_{(k+1) q}\right)_{q \in\{1, \ldots, k\}}$ are exponentially small and the fact that $\left\|\pi_{h}^{(1)} \tilde{\psi}_{k+1}\right\|_{L_{w}^{2}}=1+O\left(e^{-\frac{c}{h}}\right)$. This concludes the proof by induction. 
Step 2. Estimates on the interaction terms $\left(\left\langle\nabla u_{h}, \psi_{j}\right\rangle_{L_{w}^{2}}\right)_{j \in\{1, \ldots, n\}}$.

Lemma 100. Let us assume that the assumptions of Proposition 96 hold. Then, for $j \in\left\{1, \ldots, k_{0}\right\}$, in the limit $h \rightarrow 0$ :

$$
\left\langle\nabla u_{h}, \psi_{j}\right\rangle_{L_{w}^{2}}=C_{j} h^{p} e^{-\frac{1}{h}\left(f\left(z_{j}\right)-f\left(x_{0}\right)\right)}(1+O(h)),
$$

and for $j \in\left\{k_{0}+1, \ldots, n\right\}$, there exists $\varepsilon^{\prime}>0$ such that in the limit $h \rightarrow 0$ :

$$
\left\langle\nabla u_{h}, \psi_{j}\right\rangle_{L_{w}^{2}}=C_{j} h^{p} e^{-\frac{1}{h}\left(f\left(z_{j}\right)-f\left(x_{0}\right)\right)}(1+O(h))+O\left(e^{-\frac{1}{h}\left(f^{*}-f\left(x_{0}\right)+\varepsilon^{\prime}\right)}\right) .
$$

Proof. For $j \in\left\{1, \ldots, k_{0}\right\}$, the proof of the estimate of $\left\langle\nabla u_{h}, \psi_{j}\right\rangle_{L^{2}}$ is exactly the same as for Lemma 31. Let $j \in\left\{k_{0}+1, \ldots, n\right\}$. Using (66)-60 -63 - 64 , one has

$$
\left\langle\nabla u_{h}, \psi_{j}\right\rangle_{L_{w}^{2}}=a_{j}+b_{j}+c_{j},
$$

where $a_{j}, b_{j}$ and $c_{j}$ are defined by

$$
\begin{gathered}
a_{j}=\frac{Z_{j}^{-1}}{\left\|\pi_{h}^{(0)} \tilde{u}\right\|_{L_{w}^{2}}}\left(\left\langle\nabla \tilde{u}, \tilde{\psi}_{j}\right\rangle_{L_{w}^{2}}+\left\langle\nabla \tilde{u},\left(\pi_{h}^{(1)}-1\right) \tilde{\psi}_{j}\right\rangle_{L_{w}^{2}}\right), \\
b_{j}=\frac{Z_{j}^{-1}}{\left\|\pi_{h}^{(0)} \tilde{u}\right\|_{L_{w}^{2}}} \sum_{i=1}^{k_{0}} \kappa_{j i}\left(\left\langle\nabla \tilde{u}, \tilde{\psi}_{i}\right\rangle_{L_{w}^{2}}+\left\langle\nabla \tilde{u},\left(\pi_{h}^{(1)}-1\right) \tilde{\psi}_{i}\right\rangle_{L_{w}^{2}}\right),
\end{gathered}
$$

and

$$
c_{j}=\frac{Z_{j}^{-1}}{\left\|\pi_{h}^{(0)} \tilde{u}\right\|_{L_{w}^{2}}} \sum_{i=k_{0}+1}^{j-1} \kappa_{j i}\left(\left\langle\nabla \tilde{u}, \tilde{\psi}_{i}\right\rangle_{L_{w}^{2}}+\left\langle\nabla \tilde{u},\left(\pi_{h}^{(1)}-1\right) \tilde{\psi}_{i}\right\rangle_{L_{w}^{2}}\right),
$$

with the convention $\sum_{i=k_{0}+1}^{k_{0}}=0$. From Lemmata 97 and 99 , one has $\frac{Z_{j}^{-1}}{\left\|\pi_{h}^{(0)} \tilde{u}\right\|_{L_{w}^{2}}}=$ $1+O\left(e^{-\frac{c}{h}}\right)$. Using assumptions 2 and $4(\mathrm{a})$ in Proposition 96 and Lemma 99 , there exists $\delta_{0}>0$ such that for all $\delta \in\left(0, \delta_{0}\right)$,

$$
\begin{aligned}
a_{j}= & C_{j} h^{p} e^{-\frac{1}{h}\left(f\left(z_{j}\right)-f\left(x_{0}\right)\right)}(1+O(h))+O\left(e^{-\frac{1}{h}\left(f^{*}-f\left(x_{0}\right)+\varepsilon\right)}\right)+O\left(e^{-\frac{1}{h}\left(f^{*}-f\left(x_{0}\right)-\delta+\varepsilon\right)}\right), \\
b_{j}= & \sum_{i=1}^{k_{0}} O\left(e^{-\frac{1}{h}\left(f^{*}-f\left(z_{1}\right)+\varepsilon+f\left(z_{i}\right)-f\left(x_{0}\right)-\delta\right)}\right)+O\left(e^{-\frac{1}{h}\left(f^{*}-f\left(z_{1}\right)+\varepsilon+f\left(z_{1}\right)-f\left(x_{0}\right)-\delta+f\left(z_{i}\right)-f\left(z_{1}\right)+\varepsilon\right)}\right) \\
= & O\left(e^{-\frac{1}{h}\left(f^{*}-f\left(x_{0}\right)-\delta+\varepsilon\right)}\right) \\
c_{j}= & \sum_{i=k_{0}+1}^{j-1} O\left(e^{-\frac{1}{h}\left(f^{*}-f\left(z_{1}\right)+\varepsilon\right)}\right)\left(O\left(e^{-\frac{1}{h}\left(f\left(z_{i}\right)-f\left(x_{0}\right)-\delta\right)}\right)+O\left(e^{-\frac{1}{h}\left(f^{*}-f\left(x_{0}\right)+\varepsilon\right)}\right)\right) \\
& +O\left(e^{-\frac{1}{h}\left(f^{*}-f\left(z_{1}\right)+\varepsilon+f\left(z_{1}\right)-f\left(x_{0}\right)-\delta+f^{*}-f\left(z_{1}\right)+\varepsilon\right)}\right)=O\left(e^{-\frac{1}{h}\left(f^{*}-f\left(x_{0}\right)-\delta+\varepsilon\right)}\right) .
\end{aligned}
$$

Therefore, choosing $\delta \in(0, \varepsilon)$, there exists $\varepsilon^{\prime}>0$ such that

$$
\left\langle\nabla u_{h}, \psi_{j}\right\rangle_{L_{w}^{2}}=C_{j} h^{p} e^{-\frac{1}{h}\left(f\left(z_{j}\right)-f\left(x_{0}\right)\right)}(1+O(h))+O\left(e^{-\frac{1}{h}\left(f^{*}-f\left(x_{0}\right)+\varepsilon^{\prime}\right)}\right) .
$$

This concludes the proof of Lemma 100 . 
$\underline{\text { Step 3. Estimates on the boundary terms }}\left(\int_{\Gamma} \psi_{j} \cdot n e^{-\frac{2}{h} f} d \sigma\right)_{j \in\{1, \ldots, n\}, \Gamma \in\left\{\Sigma, \Sigma_{1}, \ldots, \Sigma_{n}\right\}}$.

Lemma 101. Let us assume that the assumptions of Proposition 96 hold. Then, there exists $\varepsilon>0$ such that in the limit $h \rightarrow 0$ one has:

- If $k \in\left\{1, \ldots, k_{0}\right\}$ and $j \in\left\{1, \ldots, k_{0}\right\}$,

$$
\begin{aligned}
\int_{\Sigma_{k}} \psi_{j} \cdot n e^{-\frac{2}{h} f} d \sigma= & \delta_{j, k} B_{k} h^{m} e^{-\frac{1}{h} f\left(z_{k}\right)}(1+O(h))+O\left(e^{-\frac{1}{h}\left(2 f\left(z_{k}\right)-f\left(z_{j}\right)+\varepsilon\right)}\right) \\
& +1_{j>k} O\left(e^{-\frac{1}{h}\left(f\left(z_{k}\right)+\varepsilon\right)}\right) .
\end{aligned}
$$

- If $k \in\left\{1, \ldots, k_{0}\right\}$ and $j \in\left\{k_{0}+1, \ldots, n\right\}, \int_{\Sigma_{k}} \psi_{j} \cdot n e^{-\frac{2}{h} f} d \sigma=O\left(e^{-\frac{1}{h}\left(f\left(z_{k}\right)+\varepsilon\right)}\right)$.

- If $k \in\left\{k_{0}+1, \ldots, n\right\}$ and $j \in\left\{1, \ldots, k_{0}\right\}, \int_{\Sigma_{k}} \psi_{j} \cdot n e^{-\frac{2}{h} f} d \sigma=O\left(e^{-\frac{1}{h}\left(2 f\left(z_{k_{0}}\right)-f\left(z_{j}\right)+\varepsilon\right)}\right)$.

- If $k \in\left\{k_{0}+1, \ldots, n\right\}$ and $j \in\left\{k_{0}+1, \ldots, n\right\}$,

$$
\int_{\Sigma_{k}} \psi_{j} \cdot n e^{-\frac{2}{h} f} d \sigma=\delta_{j, k} O\left(h^{m} e^{-\frac{1}{h} f\left(z_{k}\right)}\right)+O\left(e^{-\frac{1}{h}\left(f\left(z_{k_{0}+1}\right)+\varepsilon\right)}\right) .
$$

Proof. For all $(j, k) \in\{1, \ldots, n\}^{2}$, using $63-$-64) and Lemmata 99 and 97 together with assumption 4(b) in Proposition 96, one has in the limit $h \rightarrow 0$ :

$$
\begin{aligned}
\int_{\Sigma_{k}} \psi_{j} \cdot n e^{-\frac{2}{h} f} d \sigma= & Z_{j}^{-1}\left[\int_{\Sigma_{k}} \tilde{\psi}_{j} \cdot n e^{-\frac{2}{h} f} d \sigma+\int_{\Sigma_{k}}\left(\pi_{h}^{(1)}-1\right) \tilde{\psi}_{j} \cdot n e^{-\frac{2}{h} f} d \sigma\right] \\
& +Z_{j}^{-1} \sum_{i=1}^{j-1} \kappa_{j i}\left(\int_{\Sigma_{k}} \tilde{\psi}_{i} \cdot n e^{-\frac{2}{h} f} d \sigma+\int_{\Sigma_{k}}\left(\pi_{h}^{(1)}-1\right) \tilde{\psi}_{i} \cdot n e^{-\frac{2}{h} f} d \sigma\right) \\
= & \delta_{j, k} B_{k} h^{m} e^{-\frac{1}{h} f\left(z_{k}\right)}(1+O(h))+\frac{1}{h}\left\|\left(1-\pi_{h}^{(1)}\right) \tilde{\psi}_{j}\right\|_{H_{w}^{1}} O\left(e^{-\frac{1}{h} f\left(z_{k}\right)}\right) \\
& +\sum_{i=1}^{j-1} \kappa_{j i} \delta_{i, k} O\left(h^{m} e^{-\frac{1}{h} f\left(z_{k}\right)}\right)+\frac{\kappa_{j i}}{h}\left\|\left(1-\pi_{h}^{(1)}\right) \tilde{\psi}_{i}\right\|_{H_{w}^{1}} O\left(e^{-\frac{1}{h} f\left(z_{k}\right)}\right) .
\end{aligned}
$$

Let us know deal separately with the two cases $k \in\left\{1, \ldots, k_{0}\right\}$ and $k \in\left\{k_{0}+1, \ldots, n\right\}$. In the following, we use assumption 2(a) in Proposition 96 and Lemma 99 to estimate 264.

Case 1: $k \in\left\{1, \ldots, k_{0}\right\}$.

If $j \in\left\{1, \ldots, k_{0}\right\}$, from (264), one gets in the limit $h \rightarrow 0$ :

$$
\begin{aligned}
\int_{\Sigma_{k}} \psi_{j} \cdot n e^{-\frac{2}{h} f} d \sigma= & \delta_{j, k} B_{k} h^{m} e^{-\frac{1}{h} f\left(z_{k}\right)}(1+O(h))+O\left(e^{-\frac{1}{h}\left(f^{*}-f\left(z_{j}\right)+f\left(z_{k}\right)+\varepsilon\right)}\right) \\
& +\sum_{i=1}^{j-1} \delta_{i, k} O\left(e^{-\frac{1}{h}\left(f\left(z_{k}\right)+\varepsilon\right)}\right)+\sum_{i=1}^{j-1} O\left(e^{-\frac{1}{h}\left(f\left(z_{j}\right)-f\left(z_{i}\right)+f^{*}-f\left(z_{i}\right)+f\left(z_{k}\right)+\varepsilon\right)}\right) .
\end{aligned}
$$


Since $f^{*} \geq f\left(z_{k}\right)$ for all $k \in\left\{1, \ldots, k_{0}\right\}$ and since there exists $i<j$ such that $\delta_{i, k}=1$ if and only if $j>k$, there exists $\varepsilon>0$ such that for all $(k, j) \in\left\{1, \ldots, k_{0}\right\}^{2}$ and for all $h$ small enough,

$\int_{\Sigma_{k}} \psi_{j} \cdot n e^{-\frac{2}{h} f} d \sigma=\delta_{j, k} B_{k} h^{m} e^{-\frac{1}{h} f\left(z_{k}\right)}(1+O(h))+O\left(e^{-\frac{1}{h}\left(2 f\left(z_{k}\right)-f\left(z_{j}\right)+\varepsilon\right)}\right)+1_{j>k} O\left(e^{-\frac{1}{h}\left(f\left(z_{k}\right)+\varepsilon\right)}\right)$.

If $j \in\left\{k_{0}+1, \ldots, n\right\}$, from 264, one gets

$$
\int_{\Sigma_{k}} \psi_{j} \cdot n e^{-\frac{2}{h} f} d \sigma=O\left(e^{-\frac{1}{h}\left(f^{*}-f\left(z_{1}\right)+f\left(z_{k}\right)+\varepsilon\right)}\right)+\sum_{i=1}^{j-1} O\left(e^{-\frac{1}{h}\left(f\left(z_{k}\right)+\varepsilon\right)}\right) .
$$

Case 2: $k \in\left\{k_{0}+1, \ldots, n\right\}$.

If $j \in\left\{1, \ldots, k_{0}\right\}$, from (264) and since $f\left(z_{k}\right) \geq f^{*} \geq f\left(z_{k_{0}}\right)$, one has

$$
\begin{aligned}
\int_{\Sigma_{k}} \psi_{j} \cdot n e^{-\frac{2}{h} f} d \sigma & =O\left(e^{-\frac{1}{h}\left(f^{*}-f\left(z_{j}\right)+\varepsilon+f\left(z_{k}\right)\right)}\right)+\sum_{i=1}^{j-1} O\left(e^{-\frac{1}{h}\left(f^{*}+f\left(z_{k}\right)+f\left(z_{j}\right)-2 f\left(z_{i}\right)+\varepsilon\right)}\right) \\
& =O\left(e^{-\frac{1}{h}\left(2 f\left(z_{k_{0}}\right)-f\left(z_{j}\right)+\varepsilon\right)}\right) .
\end{aligned}
$$

If $j \in\left\{k_{0}+1, \ldots, n\right\}$, from 264, one gets

$$
\int_{\Sigma_{k}} \psi_{j} \cdot n e^{-\frac{2}{h} f} d \sigma=\delta_{j, k} O\left(h^{m} e^{-\frac{1}{h} f\left(z_{k}\right)}\right)+O\left(e^{-\frac{1}{h}\left(f\left(z_{k}\right)+\varepsilon\right)}\right),
$$

which leads to the desired estimate since $f\left(z_{k}\right) \geq f\left(z_{k_{0}+1}\right)$. This concludes the proof of Lemma 101.

Lemma 102. Let us assume that the assumptions of Proposition 96 hold. Then, for $j \in\left\{1, \ldots, k_{0}\right\}$ one has when $h \rightarrow 0$ :

$$
\int_{\Sigma} \psi_{j} \cdot n e^{-\frac{2}{h} f} d \sigma=\delta_{j_{0}, j} C^{*} h^{q^{*}} e^{-\frac{1}{h}\left(2 f^{*}-f\left(z_{j_{0}}\right)\right)}(1+O(h))+O\left(e^{-\frac{1}{h}\left(2 f^{*}-f\left(z_{j}\right)+\varepsilon\right)}\right)
$$

and for $j \in\left\{k_{0}+1, \ldots, n\right\}$ one has $\int_{\Sigma} \psi_{j} \cdot n e^{-\frac{2}{h} f} d \sigma=O\left(e^{-\frac{1}{h}\left(2 f^{*}-f\left(z_{1}\right)+\varepsilon\right)}\right)$.

Proof. Let $j \in\{1, \ldots, n\}$. Using (63)-64) and Lemmata 99 and 97 together with assumption 4(c) in Proposition 96, one has

$$
\begin{aligned}
\int_{\Sigma} \psi_{j} \cdot n e^{-\frac{2}{h} f} d \sigma= & Z_{j}^{-1}\left[\int_{\Sigma} \tilde{\psi}_{j} \cdot n e^{-\frac{2}{h} f} d \sigma+\int_{\Sigma}\left(\pi_{h}^{(1)}-1\right) \tilde{\psi}_{j} \cdot n e^{-\frac{2}{h} f} d \sigma\right] \\
& +Z_{j}^{-1} \sum_{i=1}^{j-1} \kappa_{j i}\left(\int_{\Sigma} \tilde{\psi}_{i} \cdot n e^{-\frac{2}{h} f} d \sigma+\int_{\Sigma}\left(\pi_{h}^{(1)}-1\right) \tilde{\psi}_{i} \cdot n e^{-\frac{2}{h} f} d \sigma\right) \\
= & \delta_{j_{0}, j} C^{*} h^{q^{*}} e^{-\frac{1}{h}\left(2 f^{*}-f\left(z_{j_{0}}\right)\right)}(1+O(h))+\frac{1}{h}\left\|\left(1-\pi_{h}^{(1)}\right) \tilde{\psi}_{j}\right\|_{H_{w}^{1}} O\left(e^{-\frac{1}{h} f^{*}}\right) \\
& +\sum_{i=1}^{j-1} \delta_{j_{0}, i} \kappa_{j i} O\left(h^{q^{*}} e^{-\frac{1}{h}\left(2 f^{*}-f\left(z_{j_{0}}\right)\right)}\right)+\frac{\kappa_{j i}}{h}\left\|\left(1-\pi_{h}^{(1)}\right) \tilde{\psi}_{i}\right\|_{H_{w}^{1}} O\left(e^{-\frac{1}{h} f^{*}}\right) .
\end{aligned}
$$


Let us first deal with the case $j \in\left\{1, \ldots, k_{0}\right\}$. Using assumption 2(a) in Proposition 96 and Lemma 99 , one gets from (265)

$$
\begin{aligned}
\int_{\Sigma} \psi_{j} \cdot n e^{-\frac{2}{h} f} d \sigma= & \delta_{j_{0}, j} C^{*} h^{q^{*}} e^{-\frac{1}{h}\left(2 f^{*}-f\left(z_{j_{0}}\right)\right)}(1+O(h))+O\left(e^{-\frac{1}{h}\left(f^{*}-f\left(z_{j}\right)+\varepsilon+f^{*}\right)}\right) \\
& +\sum_{i=1}^{j-1} O\left(e^{-\frac{1}{h}\left(2 f^{*}+f\left(z_{j}\right)-2 f\left(z_{i}\right)+\varepsilon\right)}\right) \\
= & \delta_{j_{0}, j} C^{*} h^{q^{*}} e^{-\frac{1}{h}\left(2 f^{*}-f\left(z_{j_{0}}\right)\right)}(1+O(h))+O\left(e^{-\frac{1}{h}\left(2 f^{*}-f\left(z_{j}\right)+\varepsilon\right)}\right) .
\end{aligned}
$$

Let us now deal with the case $j \in\left\{k_{0}+1, \ldots, n\right\}$. In that case, one obtains from 265), assumption 2(a) in Proposition 96, Lemma 99 together with the fact that $f^{*} \geq f\left(z_{i}\right)$ for all $i \in\left\{1, \ldots, k_{0}\right\}$ and $f^{*} \geq f\left(z_{k_{0}}\right) \geq f\left(z_{j_{0}}\right)$,

$$
\begin{aligned}
\int_{\Sigma} \psi_{j} \cdot n e^{-\frac{2}{h} f} d \sigma= & O\left(e^{-\frac{1}{h}\left(f^{*}-f\left(z_{1}\right)+\varepsilon+f^{*}\right)}\right)+\sum_{i=1}^{j-1} \delta_{j_{0}, i} O\left(e^{-\frac{1}{h}\left(f^{*}-f\left(z_{1}\right)+2 f^{*}-f\left(z_{j_{0}}\right)+\varepsilon\right)}\right) \\
& +\sum_{i=1}^{k_{0}} O\left(e^{-\frac{1}{h}\left(2 f^{*}-f\left(z_{1}\right)+f^{*}-f\left(z_{i}\right)+\varepsilon\right)}\right)+\sum_{i=k_{0}+1}^{j-1} O\left(e^{-\frac{1}{h}\left(2 f^{*}-2 f\left(z_{1}\right)+\varepsilon+f^{*}\right)}\right) \\
= & O\left(e^{-\frac{1}{h}\left(2 f^{*}-f\left(z_{1}\right)+\varepsilon\right)}\right) .
\end{aligned}
$$

This concludes the proof of Lemma 102 .

Step 4. Estimates on the boundary terms $\left(\int_{\Gamma}\left(\partial_{n} u_{h}\right) e^{-\frac{2}{h} f} d \sigma\right)_{\Gamma \in\left\{\Sigma, \Sigma_{1}, \ldots, \Sigma_{n}\right\}}$.

We are now in position to conclude the proof of Proposition 96 .

Proof. Let us assume that the assumptions of Proposition 96 hold. The proof is divided into two cases.

Case 1: $\Gamma=\Sigma_{k}$ in 263 for some $k \in\{1, \ldots, n\}$.

If $k \in\left\{1, \ldots, k_{0}\right\}$, from Lemmata 100 and 101 and the fact that $f^{*} \geq f\left(z_{k_{0}}\right) \geq f\left(z_{k}\right)$, one obtains that there exists $\varepsilon>0$ such that for all $j \in\{1, \ldots, n\}$, in the limit $h \rightarrow 0$

$$
\begin{aligned}
\left\langle\nabla u_{h}, \psi_{j}\right\rangle_{L_{w}^{2}} \int_{\Sigma_{k}} \psi_{j} \cdot n e^{-\frac{2}{h} f} d \sigma= & \delta_{j k} B_{k} C_{k} h^{m+p} e^{-\frac{1}{h}\left(2 f\left(z_{k}\right)-f\left(x_{0}\right)\right)}(1+O(h)) \\
& +O\left(e^{-\frac{1}{h}\left(2 f\left(z_{k}\right)-f\left(x_{0}\right)+\varepsilon\right)}\right) .
\end{aligned}
$$

Therefore, from (263), one gets for all $k \in\left\{1, \ldots, k_{0}\right\}$, in the limit $h \rightarrow 0$

$$
\int_{\Sigma_{k}}\left(\partial_{n} u_{h}\right) e^{-\frac{2}{h} f} d \sigma=B_{k} C_{k} h^{m+p} e^{-\frac{1}{h}\left(2 f\left(z_{k}\right)-f\left(x_{0}\right)\right)}(1+O(h)) .
$$

If $k \in\left\{k_{0}+1, \ldots, n\right\}$. From Lemmata 100 and 101 , one has for $j \in\left\{1, \ldots, k_{0}\right\}$ :

$$
\left\langle\nabla u_{h}, \psi_{j}\right\rangle_{L_{w}^{2}} \int_{\Sigma_{k}} \psi_{j} \cdot n e^{-\frac{2}{h} f} d \sigma=O\left(h^{p} e^{-\frac{1}{h}\left(2 f\left(z_{k_{0}}\right)-f\left(x_{0}\right)+\varepsilon\right)}\right) .
$$

and for $j \in\left\{k_{0}+1, \ldots, n\right\}$ (since $f\left(z_{j}\right) \geq f\left(z_{k_{0}+1}\right)$ and $f\left(z_{k_{0}}\right) \leq f^{*} \leq\left(z_{k_{0}+1}\right)$ ):

$$
\left\langle\nabla u_{h}, \psi_{j}\right\rangle_{L_{w}^{2}} \int_{\Sigma_{k}} \psi_{j} \cdot n e^{-\frac{2}{h} f} d \sigma=\delta_{j k} O\left(h^{p+m} e^{-\frac{1}{h}\left(2 f\left(z_{k}\right)-f\left(x_{0}\right)\right)}\right)+O\left(e^{-\frac{1}{h}\left(2 f\left(z_{k_{0}}\right)-f\left(x_{0}\right)+\varepsilon\right)}\right) .
$$


Therefore, if one assumes that $f\left(z_{k_{0}+1}\right)>f\left(z_{k_{0}}\right)$, from (263), one gets for all $k \in$ $\left\{k_{0}+1, \ldots, n\right\}$ and for all $h$ small enough

$$
\int_{\Sigma_{k}}\left(\partial_{n} u_{h}\right) e^{-\frac{2}{h} f} d \sigma=O\left(e^{-\frac{1}{h}\left(2 f\left(z_{k_{0}}\right)-f\left(x_{0}\right)+\varepsilon\right)}\right) .
$$

Case 2: $\Gamma=\Sigma$ in 263).

From 263) and using Lemmata 102 and 100 , one has

$$
\begin{aligned}
\int_{\Sigma} \partial_{n} u_{h} e^{-\frac{2}{h} f} d \sigma= & C_{j_{0}} C^{*} h^{q^{*}+p} e^{-\frac{1}{h}\left(2 f^{*}-f\left(x_{0}\right)\right)}(1+O(h))+\sum_{j=1, j \neq j_{0}}^{k_{0}} O\left(h^{p} e^{-\frac{1}{h}\left(-f\left(x_{0}\right)+2 f^{*}+\varepsilon\right)}\right) \\
& +\sum_{j=k_{0}+1}^{n} O\left(h^{p} e^{-\frac{1}{h}\left(f\left(z_{j}\right)-f\left(x_{0}\right)+2 f^{*}-f\left(z_{1}\right)+\varepsilon\right)}\right)+O\left(e^{-\frac{1}{h}\left(f^{*}-f\left(x_{0}\right)+2 f^{*}-f\left(z_{1}\right)+\varepsilon\right)}\right) \\
= & C_{j_{0}} C^{*} h^{q^{*}} e^{-\frac{1}{h}\left(2 f^{*}-f\left(x_{0}\right)\right)}(1+O(h)),
\end{aligned}
$$

which is the desired result. Proposition 96 is proved.

\subsubsection{Construction of the quasi-modes which satisfy the estimates of Propo- sition 96}

In this section, we first construct the quasi-modes $\left(\tilde{\psi}_{i}\right)_{i \in\{1, \ldots, n\}}$ and the family of quasimodes $\left(\tilde{u}=\tilde{u}_{\delta}\right)_{\delta>0}$. Then, we prove that they satisfy the estimates stated in Proposition 96. In all this section, one assumes that the hypotheses [H1], [H2] and [H3] hold. Let $\left(\Sigma_{i}\right)_{i \in\{1, \ldots, n\}}$ and $\Sigma$ be as in Proposition 96 .

Construction of the quasi-modes.

The $n+1$ quasi-modes $\left(\left(\tilde{\psi}_{i}\right)_{i \in\{1, \ldots, n\}}, \tilde{u}\right)$ are constructed as in Section 4.2 except that one adds an extra condition on the set $\Gamma_{1, j_{0}}$ used to define $\tilde{\psi}_{j_{0}}$ (where we recall that $j_{0} \in\{1, \ldots, n\}$ is such that $\left.\bar{\Sigma} \subset B_{z_{j_{0}}}\right)$. Let us be more precise on this point. Let us recall that for all $i \in\{1, \ldots, n\} \backslash\left\{j_{0}\right\}$, the 1 -form $\tilde{\psi}_{i}$ is defined as:

$$
\tilde{\psi}_{i}=e^{\frac{1}{h} f} \tilde{\phi}_{i} \in \Lambda^{1} H_{w, T}^{1}(\Omega),
$$

where the $n-1$ quasi-modes $\left(\tilde{\phi}_{i}\right)_{i \in\{1, \ldots, n\} \backslash\left\{j_{0}\right\}}$ are built in Section 4.2 (see Definition 86. Let us also recall that the function $\tilde{u}$ is the one introduced in Definition 84. The construction of $\tilde{\psi}_{j_{0}}$ requires to take into account the set $\Sigma$ in addition to the set $\Sigma_{j_{0}}$ when defining the cut off function $\chi_{j_{0}}$ in Definition 86. More precisely, and thanks to Proposition 80 , the set $\Gamma_{1, j_{0}}$ can be taken such that

$$
\bar{\Sigma}_{j_{0}} \cup \bar{\Sigma} \subset \Gamma_{1, j_{0}}
$$

Then, with $\Gamma_{1, j_{0}}$ satisfying the previous condition, the cut-off fonction $\chi_{j_{0}}$ and the 1form $\tilde{\phi}_{j_{0}} \in \Lambda^{1} H_{T}^{1}(\Omega)$ are defined exactly as in Definition 86 for $i=j_{0}$. Finally, one sets:

$$
\tilde{\psi}_{j_{0}}=e^{\frac{1}{h} f} \tilde{\phi}_{j_{0}} \in \Lambda^{1} H_{w, T}^{1}(\Omega) .
$$

The quasi-modes satisfy the estimates stated in Proposition 96. 
Using in addition to [H1]-[H2]-[H3] the hypotheses $(29)$ and $(30)$, one easily obtains that $\left(\left(\tilde{\psi}_{i}\right)_{i=1, \ldots, n}, \tilde{u}\right)$ satisfy the estimates $1,2,3,4(\mathrm{a})$ and $4(\mathrm{~b})$ stated in Proposition 96. following exactly the computations made on $\left(\left(\tilde{\phi}_{i}\right)_{i=1, \ldots, n}, \tilde{u}\right)$ in Section 4.5 . 2(a) follows from (234)-(235)-(29)-(30), 2(b) is proven in Lemma 85, 3 follows from (238)-(29), 4(b) is proven in Step 3 in Section 4.5 and 4(a) is a consequence of (242)-(243)-(244)-(29). In particular, one gets that the constants $\left(B_{i}\right)_{i=1, \ldots, n}, m,\left(C_{i}\right)_{i=1, \ldots, n}$ and $p$ in Proposition 96 are given by (241)-(245).

The following lemma deals with the assumption 4(c) in Proposition 96 which requires to use Proposition 91 .

Lemma 103. Let us assume that the hypotheses [H1], [H2] and [H3] hold. Let $j \in$ $\{1, \ldots, n\}$. Then, when $h \rightarrow 0$, one has

$$
\int_{\Sigma} \tilde{\psi}_{j} \cdot n e^{-\frac{2}{h} f} d \sigma=\delta_{j_{0}, j} \frac{B^{*} \sqrt{2}\left(\left.\operatorname{det} \operatorname{Hess} f\right|_{\partial \Omega}\left(z_{j_{0}}\right)\right)^{\frac{1}{4}}}{\pi^{\frac{d-1}{4}} \sqrt{\partial_{n} f\left(z_{j_{0}}\right)}} h^{p^{*}-\frac{d+1}{4}} e^{-\frac{1}{h}\left(2 f^{*}-f\left(z_{j_{0}}\right)\right)}(1+O(h)),
$$

where $B^{*}$ and $p^{*}$ are defined by (33).

Proof. By construction, if $j \neq j_{0}$ then $\tilde{\psi}_{j} \equiv 0$ on $B_{z_{j_{0}}}$. Let us deal with the case $j=j_{0}$. Using (266), one has

$$
\int_{\Sigma} \tilde{\psi}_{j_{0}} \cdot n e^{-\frac{2}{h} f} d \sigma=\int_{\Sigma} \tilde{\phi}_{j_{0}} \cdot n e^{-\frac{1}{h} f} d \sigma
$$

Let $u_{z_{j_{0}}, w k b}^{(1)}$ be the WKB expansion defined by 184 . Following the beginning of Section 4.4.2, let us consider

1. a neighborhood $V_{\Gamma_{S t, j_{0}}}$ of $\Sigma$ in $\bar{\Omega}$, which is stable under the dynamics (193) and such that, for some $\varepsilon>0, V_{\Gamma_{S t, j_{0}}}+B(0, \varepsilon) \subset V_{\Gamma_{1, j_{0}}} \cap\left(\dot{\Omega}_{j_{0}} \cup \Gamma_{1, j_{0}}\right)$,

2. and a cut-off function $\chi_{w k b, j_{0}} \in C_{c}^{\infty}\left(\dot{\Omega}_{j_{0}} \cup \Gamma_{1, j_{0}}\right)$ with $\chi_{w k b, j_{0}} \equiv 1$ on a neighborhood of $\overline{V_{\Gamma_{S t, j_{0}}}}$ such that $\operatorname{supp} \chi_{w k b, j_{0}} \subset V_{\Gamma_{1, j_{0}}} \cap\left(\dot{\Omega}_{j_{0}} \cup \Gamma_{1, j_{0}}\right)$.

Using Proposition 91, there exists $c_{z_{j_{0}}}(h) \in \mathbb{R}_{+}^{*}$ such that

$$
\left\|e^{\frac{1}{h} d_{a}\left(\cdot, z_{j_{0}}\right)}\left(u_{h, j_{0}}^{(1)}-c_{z_{j_{0}}}(h) u_{z_{j_{0}}, w k b}^{(1)}\right)\right\|_{H^{1}\left(V_{\Gamma_{S t, j_{0}}}\right)}=O\left(h^{\infty}\right) .
$$

Let us now introduce

$$
\tilde{\phi}_{z_{j_{0}}, w k b}:=c_{z_{j_{0}}}(h) \chi_{w k b, j_{0}} u_{z_{j_{0}}, w k b}^{(1)}
$$

so that

$$
\int_{\Sigma} \tilde{\phi}_{j_{0}} \cdot n e^{-\frac{1}{h} f} d \sigma=\int_{\Sigma} \tilde{\phi}_{z_{j_{0}}, w k b} \cdot n e^{-\frac{1}{h} f} d \sigma+\int_{\Sigma}\left(\tilde{\phi}_{j_{0}}-\tilde{\phi}_{z_{j_{0}}, w k b}\right) \cdot n e^{-\frac{1}{h} f} d \sigma .
$$

Let us first deal with the term $\int_{\Sigma} \tilde{\phi}_{z_{j_{0}}, w k b} \cdot n e^{-\frac{1}{h} f}$ in 268). Using (33), one has (since $\Phi=f, \partial_{n} \Phi=-\partial_{n} f$ and $a_{0}=1$ on $\partial \Omega$, see (189)) when $h \rightarrow 0$,

$$
\begin{aligned}
\int_{\Sigma} \tilde{\phi}_{z_{j_{0}}, w k b} \cdot n e^{-\frac{1}{h} f} d \sigma & =c_{z_{j_{0}}}(h) \int_{\Sigma} \chi_{w k b, j_{0}} u_{z_{j_{0}}, w k b}^{(1)} \cdot n e^{-\frac{1}{h} f} \\
& =2 c_{z_{j_{0}}}(h) \int_{\Sigma} \partial_{n} f e^{-\frac{1}{h}\left(2 f-f\left(z_{j_{0}}\right)\right)}(1+O(h)) \\
& =2 c_{z_{j_{0}}}(h) B^{*} h^{p^{*}} e^{-\frac{1}{h}\left(2 f^{*}-f\left(z_{j_{0}}\right)\right)}(1+O(h)) .
\end{aligned}
$$


Then using (199), one obtains in the limit $h \rightarrow 0$ :

$$
\int_{\Sigma} \tilde{\phi}_{z_{j_{0}}, w k b} \cdot n e^{-\frac{1}{h} f} d \sigma=\frac{B^{*} \sqrt{2}\left(\left.\operatorname{det} \operatorname{Hess} f\right|_{\partial \Omega}\left(z_{j_{0}}\right)\right)^{\frac{1}{4}}}{\pi^{\frac{d-1}{4}} \sqrt{\partial_{n} f\left(z_{j_{0}}\right)}} h^{p^{*}-\frac{d+1}{4}} e^{-\frac{1}{h}\left(2 f^{*}-f\left(z_{j_{0}}\right)\right)}(1+O(h)) .
$$

Let us now estimate the term $\int_{\Sigma}\left(\tilde{\phi}_{j_{0}}-\tilde{\phi}_{z_{j_{0}}, w k b}\right) \cdot n e^{-\frac{1}{h} f}$ in 268). Since $d_{a}\left(\cdot, z_{j_{0}}\right)=$ $f-f\left(z_{j_{0}}\right)=\Phi-f\left(z_{j_{0}}\right)$ on $\Sigma$, one obtains using Lemma 92 there exist $C>0, h_{0}>0$ and $\eta>0$ such that for all $h \in\left(0, h_{0}\right)$,

$$
\begin{aligned}
\left|\int_{\Sigma}\left(\tilde{\phi}_{j_{0}}-\tilde{\phi}_{z_{j_{0}}, w k b}\right) \cdot n e^{-\frac{1}{h} f} d \sigma\right|= & \left|\int_{\Sigma}\left(\frac{u_{h, j_{0}}^{(1)}}{\Theta_{j_{0}}}-c_{z_{j_{0}}}(h) u_{z_{j_{0}}, w k b}^{(1)}\right) \cdot n e^{-\frac{1}{h} f} d \sigma\right| \\
\leq & \frac{e^{-\frac{1}{h}\left(2 f^{*}-f\left(z_{j_{0}}\right)\right)}}{\Theta_{j_{0}}} \int_{\Sigma}\left|\left(u_{h, j_{0}}^{(1)}-c_{z_{j_{0}}}(h) u_{z_{j_{0}}, w k b}^{(1)}\right) e^{\frac{d_{a}\left(\cdot, z_{j_{0}}\right)}{h}} d \sigma\right| \\
& +e^{-\frac{1}{h}\left(2 f^{*}-f\left(z_{j_{0}}\right)\right)} \frac{\left|\Theta_{j_{0}}-1\right|}{\Theta_{j_{0}}}\left|c_{z_{j_{0}}}(h)\right| \int_{\Sigma}\left|u_{z_{j_{0}}, w k b}^{(1)} e^{\frac{\Phi-f\left(z_{j_{0}}\right)}{h}} d \sigma\right| \\
\leq & C e^{-\frac{1}{h}\left(2 f^{*}-f\left(z_{j_{0}}\right)\right)}|| e^{\frac{d_{a}\left(\cdot, z_{j_{0}}\right)}{h}}\left(u_{h, j_{0}}^{(1)}-c_{z_{j_{0}}}(h) u_{z_{j_{0}}, w k b}^{(1)}\right) \|_{H^{1}\left(V_{\Gamma_{S t, j_{0}}}\right)} \\
& +C e^{-\frac{1}{h}\left(2 f^{*}-f\left(z_{j_{0}}\right)\right)}\left|c_{z_{j_{0}}}(h)\right| e^{-\frac{\eta}{h}}\left\|\chi_{w k b, j_{0}} u_{z_{j_{0}}, w k b}^{(1)} e^{\frac{\Phi-f\left(z_{j_{0}}\right)}{h}}\right\|_{H^{1}\left(\dot{\Omega}_{j_{0}}\right)} .
\end{aligned}
$$

Since it holds $u_{z_{j_{0}}, w k b}^{(1)} e^{\frac{\Phi-f\left(z_{j_{0}}\right)}{h}}=d_{f-\left(\Phi-f\left(z_{j_{0}}\right)\right), h} a(\cdot, h)=h d a(\cdot, h)+\nabla(f-\Phi) \wedge a(\cdot, h)$ (see (184)), there exists $C>0$ such that for all $h$ small enough,

$$
\left\|\chi_{w k b, j_{0}} u_{z_{j_{0}}, w k b}^{(1)} e^{\frac{\Phi-f\left(z_{j_{0}}\right)}{h}}\right\|_{H^{1}\left(\dot{\Omega}_{j_{0}}\right)} \leq C .
$$

Then, one obtains using Proposition 91 and (199):

$$
e^{\frac{1}{h}\left(2 f^{*}-f\left(z_{j_{0}}\right)\right)}\left|\int_{\Sigma}\left(\tilde{\phi}_{j_{0}}-\tilde{\phi}_{z_{j_{0}}, w k b}\right) \cdot n e^{-\frac{1}{h} f}\right|=O\left(h^{\infty}\right)+C e^{-\frac{\eta}{h}} h^{-\frac{d+1}{4}}=O\left(h^{\infty}\right) .
$$

Injecting the estimates (269)-270) in 268) and using (267) imply that in the limit $h \rightarrow 0$ :

$$
\int_{\Sigma} \tilde{\psi}_{j_{0}} \cdot n e^{-\frac{2}{h} f} d \sigma=\frac{B^{*} \sqrt{2}\left(\left.\operatorname{det} \operatorname{Hess} f\right|_{\partial \Omega}\left(z_{j_{0}}\right)\right)^{\frac{1}{4}}}{\pi^{\frac{d-1}{4}} \sqrt{\partial_{n} f\left(z_{j_{0}}\right)}} h^{p^{*}-\frac{d+1}{4}} e^{-\frac{1}{h}\left(2 f^{*}-f\left(z_{j_{0}}\right)\right)}(1+O(h)) .
$$

This proves Lemma 103 .

In conclusion, the $n$ quasi-modes $\left(\tilde{\psi}_{i}\right)_{i=1, \ldots, n}$ and the family of quasi-modes $\left(\tilde{u}=\tilde{u}_{\delta}\right)_{\delta>0}$ satisfy all the conditions of Proposition 96. This concludes the proof of Theorem 2 .

\subsubsection{Proof of Corollary 17}

Let us assume that the hypotheses [H1]-[H2]-[H3] hold and let us assume that $\left.f\right|_{\partial \Omega}$ has only two local minima $z_{1}$ and $z_{2}$ such that $f\left(z_{1}\right)<f\left(z_{2}\right)$. Let $\Sigma \subset \partial \Omega$ be a smooth open set such that $\bar{\Sigma} \subset B_{z_{1}}$ and

$$
f^{*}:=\inf _{\Sigma} f
$$


In addition, let us assume that (37) and (38) hold and let us assume that $f^{*}=f\left(z_{2}\right)$. Then, the inequalities (37) and (38) are exactly $(29)$ and (30) (in the case $n=2$ with $j_{0}=1$ and $k_{0}=2$ ). Therefore, (35) holds. It remains to compute the prefactor in (35). To this end, we need the constants $B^{*}$ and $p^{*}$ in $(33)$. Let us assume that there is only one minimizer $z^{*}$ of $f$ on $\Sigma$. This implies that $z^{*} \in \partial \Sigma$ since $z_{1}$ is the only critical point of $\left.f\right|_{\partial \Omega}$ in $B_{z_{1}}$. Furthermore, we assume that $z^{*}$ is a non degenerate minimum of $f_{\mid \partial \Sigma}$ with $\partial_{n(\partial \Sigma)} f\left(z^{*}\right)<0$ where $n(\partial \Sigma)$ is the unit outward normal to $\partial \Sigma \subset \partial \Omega$. Then, using Laplace's method, in the limit $h \rightarrow 0$ :

$$
\int_{\Sigma} \partial_{n} f e^{-\frac{2}{h} f} d \sigma=-\frac{\partial_{n} f\left(z^{*}\right)(\pi h)^{\frac{d}{2}}}{2 \pi \partial_{n(\partial \Sigma)} f\left(z^{*}\right) \sqrt{\operatorname{det} \operatorname{Hess} f_{\mid \partial \Sigma}\left(z^{*}\right)}} e^{-\frac{2}{h} f^{*}}(1+O(h)),
$$

with by convention, $\operatorname{det} \operatorname{Hess} f_{\mid \partial \Sigma}\left(z^{*}\right)=1$ if $d=2$. This specifies the constants $B^{*}$ and $p^{*}$ appearing in (33). This ends the proof of Corollary 17 .

\section{Acknowledgements}

The authors would like to thank Guy Barles, Bernard Helffer, François Laudenbach, Francis Nier and Benoit Perthame for fruitful discussions on preliminary versions of this work. This work is supported by the European Research Council under the European Union's Seventh Framework Programme (FP/2007-2013) / ERC Grant Agreement number 614492 .

\section{Main notation used in this work}

- $\left(\tau_{\Omega}, X_{\tau_{\Omega}}\right)$, p. 4

- $\mathbb{P}_{x}$, p. 7

- $\nu_{h}$, p. 8

- $L_{f, h}^{(0)}$ and $L_{f, h}^{D,(0)}(\Omega)$, p. 9

- $\lambda_{h}$, p. 10 (see also p. 29 )

- $u_{h}$, p. 10 (see also p. 29 )

- $\left\{k_{0,1}, \ldots, k_{0, n}\right\}$, p. 11

- $g$, p. 12

- $L(\gamma, I)$, p. 12

- $d_{a}$, p. 12

- $x_{0}$, p. 13

- $\left\{z_{1}, \ldots, z_{n}\right\}$, p. 13

- Hypotheses [H1], [H2], and [H3], p. 13

- $n_{0}$, p. 13

- $B_{z_{i}}$ and $B_{z_{i}}^{c}$, p. 13
- $\Sigma_{i}$, p. 15

- $f^{*}$, p. 22

- $B^{*}$ and $p^{*}$, p. 22

- $z^{*}$, p. 24

- $\Lambda^{p} C^{\infty}(\bar{\Omega})$, p. 26

- $\Lambda^{p} C_{T}^{\infty}(\bar{\Omega})$ and $\Lambda^{p} C_{N}^{\infty}(\bar{\Omega})$, p. 26

- $\Lambda^{p} L_{w}^{2}(\Omega)$ and $\Lambda^{p} H_{w}^{q}(\Omega)$, p. 26

- $\Lambda^{p} H_{w, T}^{q}(\Omega)$ and $\Lambda^{p} H_{w, N}^{q}(\Omega)$, p. 26

- $\Lambda^{p} L^{2}(\Omega)$ and $\Lambda^{p} H^{q}(\Omega)$, p. 27

- $\Lambda^{p} H_{T}^{q}(\Omega)$ and $\Lambda^{p} H_{N}^{q}(\Omega)$, p. 27

- $\|\cdot\|_{H_{w}^{q}}$ and $\langle,\rangle_{L_{w}^{2}}$, p. 27

- \|\|$_{H^{q}}$ and $\langle,\rangle_{L^{2}}$, p. 27

- $\Delta_{f, h}^{(p)}, \mathrm{p} \cdot 27$

- $\Delta_{f, h}^{D,(p)}(\Omega)$ and $\Delta_{f, h}^{N,(p)}(\Omega)$, p. 28

- $L_{f, h}^{D,(p)}(\Omega)$, p. 28

- $\pi_{E}(A)$, p. 29 
- For $p \in\{0,1\}, \pi_{h}^{(p)}$, p. 30

- $\tilde{u}$, p. 31 (construction 92 )

- $p, m, C_{i}$, and $B_{i}$, p. 31 (explicit values p. 123 and 124)

- $\tilde{\psi}_{i}$, p. 31

- $\tilde{\phi}_{i}$, p. 32 (construction p. 93 )

- $\kappa_{j i}$, p. 34

- $Z_{j}$ and $\psi_{j}$ p. 35

- $A(x, y)$, p. 40

- $d_{a}^{\partial \Omega}, \mathrm{p} .52$

- $\left\{x_{1}, \ldots, x_{m}\right\}$, p. 56

- $V_{\partial \Omega}$, p. 66

- $f_{-}, \mathrm{p} .67$

- $\dot{\Omega}$, p. 72

- $\Lambda^{p} H_{d}(\dot{\Omega})$ and $\Lambda^{p} H_{d^{*}}(\dot{\Omega})$, p. 72

- $\Gamma_{T}$ and $\Gamma_{N}$, p. 75 (see also p. 88)

- $\Lambda^{p} H_{d, \Gamma}(\dot{\Omega})$ and $\Lambda^{p} H_{d^{*}, \Gamma}(\dot{\Omega})$, p. 76

- $d_{T}^{(p)}(\dot{\Omega})$ and $\delta_{N}^{(p)}(\dot{\Omega})$, p. 77

- $\Delta_{f, h}^{M,(p)}(\dot{\Omega})$, p. 77

- $\mathcal{Q}_{f, h}^{M,(p)}(\dot{\Omega})$, p. 78

- $x_{d}$, p. 80

- $\Psi_{i}, f_{+, i}$, and $f_{-, i}$, p. 81

- $\Sigma_{a}(a>0)$, p. 81

- $\Gamma_{1, i}$, p. 81

- $V_{\Gamma_{1, i}}$, p. 81
- $\dot{\Omega}_{i}$, p. 84

- $\Omega_{0}, \Gamma_{0}$, p. 84

- $\Gamma_{2, i}$, p. 84

- $\mathcal{S}_{M, i}$, p. 86

- When the index $i \in\{1, \ldots, n\}$ is dropped (p. 87): $z=z_{i}, \Gamma_{1}=\Gamma_{1, i}$, $\Gamma_{2}=\Gamma_{2, i}, \dot{\Omega}=\dot{\Omega}_{i}, V_{\Gamma_{1}}=V_{\Gamma_{1, i}}$, $\Psi=\Psi_{i}, f_{+}=f_{+, i}$ and $f_{-}=f_{-, i}$.

- $u_{w k b}^{(0)}$, p. 104

- $u_{w k b}^{(1)}, \mathrm{p} \cdot 104$

- $u_{z, w k b}^{(1)}$, p. 104

- $C_{z, w k b}$, p. 105

- $V_{\Gamma_{s t}}$ and $V_{\Gamma_{s t}^{\prime}}$, p. 107

- $\kappa$, p. 108

- $c_{z}(h)$, p. 108

- $\Theta_{i}$, p. 118

- $\tilde{\phi}_{z_{i}, w k b}, \mathrm{p} .121$

- $K_{\alpha}$, p. 128

- $\tilde{u}_{h}$, p. 128

- $Z_{h}(O)$, p. 128

- $w_{h}$, p. 128

- $\Sigma$ and $j_{0}$, p. 132

- $k_{0}$, p. 132

- $C^{*}$ and $q^{*}$, p. 132

- $\chi_{j_{0}}$ and $\tilde{\phi}_{j_{0}}$, p. 140

\section{References}

[1] L. Ambrosio, N. Fusco, and D. Pallara. Functions of bounded variation and free discontinuity problems. Oxford science publications, 2000.

[2] N. Berglund. Kramers' law: Validity, derivations and generalisations. Markov Processes Relat. Fields, 19:459-490, 2013.

[3] A. Binder, T. Lelièvre, and G. Simpson. A generalized parallel replica dynamics. Journal of Computational Physics, 284:595-616, 2015. 
[4] F. Bouchet and J. Reygner. Generalisation of the Eyring-Kramers transition rate formula to irreversible diffusion processes. Annales Henri Poincaré, 17(12):3499$3532,2016$.

[5] A. Bovier, M. Eckhoff, V. Gayrard, and M. Klein. Metastability in reversible diffusion processes. I. sharp asymptotics for capacities and exit times. J. Eur. Math. Soc. (JEMS), 6:399-424, 2004.

[6] A. Bovier, V. Gayrard, and M. Klein. Metastability in reversible diffusion processes. II. precise asymptotics for small eigenvalues. J. Eur. Math. Soc. (JEMS), 7:69-99, 2005 .

[7] G.R. Bowman, V.S. Pande, and F. Noé. An Introduction to Markov State Models and Their Application to Long Timescale Molecular Simulation. Springer, 2014.

[8] R. Brown. The mixed problem for Laplace's equation in a class of Lipschitz domains. Communications in partial differential equations, 19(7-8):1217-1233, 1994.

[9] M. Cameron. Computing the asymptotic spectrum for networks representing energy landscapes using the minimum spanning tree. Netw. Heterog. Media, 9(3):383-416, 2014 .

[10] P. Cattiaux, P. Collet, A. Lambert, S. Martínez, S. Méléard, J. San Martín, et al. Quasi-stationary distributions and diffusion models in population dynamics. The Annals of Probability, 37(5):1926-1969, 2009.

[11] N. Champagnat and D. Villemonais. General criteria for the study of quasistationarity. arXiv preprint arXiv:1712.08092, 2017.

[12] K.C. Chang and J. Liu. A cohomology complex for manifolds with boundary. Topological methods in non linear analysis, 5:325-340, 1995.

[13] P. Collet, S. Martínez, and J. San Martín. Quasi-Stationary Distributions. Springer, 2013.

[14] H.L. Cycon, R.G. Froese, W. Kirsch, and B. Simon. Schrödinger operators with application to quantum mechanics and global geometry. Springer, 1987.

[15] E.B. Davies. Metastable states of symmetric Markov semigroups I. Proc. London Math. Soc., 45(3):133-150, 1982.

[16] E.B. Davies. Metastable states of symmetric Markov semigroups II. J. London Math. Soc., 26(3):541-556, 1982.

[17] M.V. Day. On the exponential exit law in the small parameter exit problem. Stochastics: An International Journal of Probability and Stochastic Processes, 8(4):297-323, 1983.

[18] M.V. Day. On the asymptotic relation between equilibrium density and exit measure in the exit problem. Stochastics: an international journal of probability and stochastic processes, 12(3-4):303-330, 1984. 
[19] M.V. Day. Recent progress on the small parameter exit problem. Stochastics: An International Journal of Probability and Stochastic Processes, 20(2):121-150, 1987.

[20] M.V. Day. Mathematical approaches to the problem of noise-induced exit. In W.M. McEneaney, G.G. Yin, and Q. Zhang, editors, Stochastic Analysis, Control, Optimization and Applications: A Volume in Honor of W.H. Fleming, pages 269287. Birkhäuser, 1999.

[21] A. Devinatz and A. Friedman. Asymptotic behavior of the principal eigenfunction for a singularly perturbed Dirichlet problem. Indiana Univ. Math. J., 27:143-157, 1978.

[22] A. Devinatz and A. Friedman. The asymptotic behavior of the solution of a singularly perturbed Dirichlet problem. Indiana Univ. Math. J., 27(3):527-537, 1978.

[23] G. Di Gesù, T. Lelièvre, D. Le Peutrec, and B. Nectoux. Jump markov models and transition state theory: the quasi-stationary distribution approach. Faraday Discussions, 195:469-495, 2017.

[24] G. Di Gesù, T. Lelièvre, D. Le Peutrec, and B. Nectoux. The exit from a metastable state: concentration of the exit point distribution on the low energy saddle points, 2019. In preparation.

[25] M. Dimassi and J. Sjöstrand. Spectral asymptotics in the semi-classical limit. Number 268. Cambridge university press, 1999.

[26] M. Eckhoff. Precise asymptotics of small eigenvalues of reversible diffusions in the metastable regime. Ann. Probab., 33(1):244-299, 2005.

[27] A. Eizenberg. The exponential leveling and the Ventcel-Freidlin "minimal action" function. Journal d'Analyse Mathématique, 54(1):99-111, 1990.

[28] L.C. Evans. Partial differential equations, volume 19 of Graduate Studies in Mathematics. American Mathematical Society, Providence, RI, second edition, 2010.

[29] L.C. Evans and R.F. Gariepy. Measure theory and fine properties of functions. Studies in Advanced Mathematics. CRC Press, 1992.

[30] Y. Fan, S. Yip, and B. Yildiz. Autonomous basin climbing method with sampling of multiple transition pathways: application to anisotropic diffusion of point defects in hcp Zr. Journal of Physics: Condensed Matter, 26:365402, 2014.

[31] M.I. Freidlin and A.D. Wentzell. Random Perturbations of Dynamical Systems. Springer-Verlag, 1984.

[32] A. Galves, E. Olivieri, and M.E. Vares. Metastability for a class of dynamical systems subject to small random perturbations. The Annals of Probability, pages 1288-1305, 1987.

[33] V. Gol'dshtein, I. Mitrea, and M. Mitrea. Hodge decompositions with mixed boundary conditions and applications to partial differential equations on Lipschitz manifolds. Journal of Mathematical Sciences, 172(3):347-400, 2011. 
[34] P. Hänggi, P. Talkner, and M. Borkovec. Reaction-rate theory: fifty years after Kramers. Reviews of Modern Physics, 62(2):251-342, 1990.

[35] B. Helffer. Introduction to the semiclassical analysis for the Schrödinger operator and applications. Springer lecture Notes in Math, 1988.

[36] B. Helffer. Semi-classical analysis for the Schrödinger operator and applications, volume 1336. Springer, 2006.

[37] B. Helffer. Spectral theory and its applications, volume 139. Cambridge University Press, 2013.

[38] B. Helffer, M. Klein, and F. Nier. Quantitative analysis of metastability in reversible diffusion processes via a Witten complex approach. Mat. Contemp., 26:41-85, 2004.

[39] B. Helffer and F. Nier. Quantitative analysis of metastability in reversible diffusion processes via a Witten complex approach: the case with boundary. Mémoire de la Société mathématique de France, 105:1-89, 2006.

[40] B. Helffer and J. Sjöstrand. Multiple wells in the semi-classical limit I. Comm. Partial Differential Equations, 9(4):337-408, 1984.

[41] B. Helffer and J. Sjöstrand. Multiple wells in the semi-classical limit III-interaction through non-resonant wells. Mathematische Nachrichten, 124(1):263-313, 1985.

[42] B. Helffer and J. Sjöstrand. Puits multiples en limite semi-classique. II. Interaction moléculaire. Symétries. Perturbation. Annales de l'IHP Physique théorique, 42(2):127-212, 1985.

[43] B. Helffer and J. Sjöstrand. Puits multiples en mécanique semi-classique, IV: Etude du complexe de Witten. Comm. Partial Differential Equations, 10(3):245-340, 1985 .

[44] B. Helffer and J. Sjöstrand. Résonances en limite semi-classique. Mémoires de la Société Mathématique de France, 24:1-228, 1986.

[45] R.A. Holley, S. Kusuoka, and D.W. Stroock. Asymptotics of the spectral gap with applications to the theory of simulated annealing. J. Funct. Anal., 83(2):333-347, 1989.

[46] T. Jakab, I. Mitrea, and M. Mitrea. On the regularity of differential forms satisfying mixed boundary conditions in a class of Lipschitz domains. Indiana University mathematics journal, 58(5):2043-2071, 2009.

[47] S. Kamin. Elliptic perturbation of a first order operator with a singular point of attracting type. Indiana Univ. Math. J., 27(6):935-952, 1978.

[48] C. Kipnis and C.M. Newman. The metastable behavior of infrequently observed, weakly random, one-dimensional diffusion processes. SIAM J. Appl. Math., 45(6):972-982, 1985.

[49] H.A. Kramers. Brownian motion in a field of force and the diffusion model of chemical reactions. Physica, 7(4):284-304, 1940. 
[50] C. Landim. Metastable Markov chains. arXiv preprint arXiv:180\%.04144, 2018.

[51] C. Landim, M. Mariani, and I. Seo. A Dirichlet and a Thomson principle for nonselfadjoint elliptic operators, metastability in non-reversible diffusion processes. Archive for Rational Mechanics and Analysis, 2018.

[52] F. Laudenbach. A Morse complex on manifolds with boundary. Geometriae Dedicata, 153(1):47-57, 2011.

[53] C. Le Bris, T. Lelièvre, M. Luskin, and D. Perez. A mathematical formalization of the parallel replica dynamics. Monte Carlo Methods and Applications, 18(2):119$146,2012$.

[54] D. Le Peutrec. Small eigenvalues of the Neumann realization of the semiclassical Witten Laplacian. Ann. Fac. Sci. Toulouse Math. (6), 19(3-4):735-809, 2010.

[55] T. Lelièvre, D. Le Peutrec, and B. Nectoux. Exit event from a metastable state and Eyring-Kramers law for the overdamped Langevin dynamics. hal-01696187, 2018.

[56] T. Lelièvre and F. Nier. Low temperature asymptotics for quasistationary distributions in a bounded domain. Anal. PDE, 8(3):561-628, 2015.

[57] P.-L. Lions. Generalized solutions of Hamilton-Jacobi equations, volume 69. Pitman Publishing, 1982.

[58] R.S. Maier and D.L. Stein. Escape problem for irreversible systems. Phys. Rev. E, 48:931-938, 1993.

[59] R.S. Maier and D.L. Stein. Limiting exit location distributions in the stochastic exit problem. SIAM Journal on Applied Mathematics, 57(3):752-790, 1997.

[60] R. Marcelin. Contribution à l'étude de la cinétique physico-chimique. Ann. Physique, 3:120-231, 1915.

[61] P. Mathieu. Zero white noise limit through Dirichlet forms, with application to diffusions in a random medium. Probability Theory and Related Fields, 99(4):549$580,1994$.

[62] P. Mathieu. Spectra, exit times and long time asymptotics in the zero-whitenoise limit. Stochastics: An International Journal of Probability and Stochastic Processes, 55(1-2):1-20, 1995.

[63] B.J. Matkowsky and Z. Schuss. The exit problem for randomly perturbed dynamical systems. SIAM J. Appl. Math., 33(2):365-382, 1977.

[64] L. Miclo. Comportement de spectres d'opérateurs de Schrödinger à basse température. Bulletin des sciences mathématiques, 119(6):529-554, 1995.

[65] M. Mitrea. Dirichlet integrals and Gaffney-Friedrichs inequalities in convex domains. In Forum Mathematicum, volume 13, pages 531-568. Walter de Gruyter and co., 2001. 
[66] T. Naeh, M.M. Klosek, B.J. Matkowsky, and Z. Schuss. A direct approach to the exit problem. SIAM J. Appl. Math., 50(2):595-627, 1990.

[67] F. Nier. Boundary conditions and subelliptic estimates for geometric KramersFokker-Planck operators on manifolds with boundaries, volume 252. Memoirs of the American Mathematical Society, 2018.

[68] B. Perthame. Perturbed dynamical systems with an attracting singularity and weak viscosity limits in Hamilton-Jacobi equations. Transactions of the American Mathematical Society, 317(2):723-748, 1990.

[69] C. Pugh. Real mathematical analysis, volume 2011. Springer, 2002.

[70] Z. Schuss. Theory and applications of stochastic processes: an analytical approach, volume 170. Springer, 2009.

[71] Z. Schuss and B.J. Matkowsky. The exit problem: a new approach to diffusion across potential barriers. SIAM Journal on Applied Mathematics, 36(3):604-623, 1979.

[72] C. Schütte. Conformational dynamics: modelling, theory, algorithm and application to biomolecules, 1998. Habilitation dissertation, Free University Berlin.

[73] C. Schütte and M. Sarich. Metastability and Markov state models in molecular dynamics, volume 24 of Courant Lecture Notes. American Mathematical Society, 2013.

[74] G. Schwarz. Hodge decomposition - a method for solving boundary value problems, volume 1607 of Lecture Notes in Mathematics. Springer-Verlag, Berlin, 1995.

[75] B. Simon. Semiclassical analysis of low lying eigenvalues, ii. tunneling. Ann. of Math., pages 89-118, 1984.

[76] M.R. Sorensen and A.F. Voter. Temperature-accelerated dynamics for simulation of infrequent events. J. Chem. Phys., 112(21):9599-9606, 2000.

[77] M. Sugiura. Metastable behaviors of diffusion processes with small parameter. Journal of the Mathematical Society of Japan, 47(4):755-788, 1995.

[78] G.H. Vineyard. Frequency factors and isotope effects in solid state rate processes. Journal of Physics and Chemistry of Solids, 3(1):121-127, 1957.

[79] A.F. Voter. A method for accelerating the molecular dynamics simulation of infrequent events. J. Chem. Phys., 106(11):4665-4677, 1997.

[80] A.F. Voter. Parallel replica method for dynamics of infrequent events. Phys. Rev. $B, 57(22): \mathrm{R} 13$ 985, 1998.

[81] A.F. Voter. Radiation Effects in Solids, chapter Introduction to the Kinetic Monte Carlo Method. Springer, NATO Publishing Unit, 2005.

[82] D.J. Wales. Energy landscapes. Cambridge University Press, 2003.

[83] E. Witten. Supersymmetry and Morse theory. J. Differential Geom., 17(4):661$692,1982$. 